\title{
Non-invasive methods for the assessment of wall shear rate and arterial impedance
}

Citation for published version (APA):

Brands, P. J. (1996). Non-invasive methods for the assessment of wall shear rate and arterial impedance. [Doctoral Thesis, Maastricht University]. Rijksuniversiteit Limburg.

https://doi.org/10.26481/dis.19960531pb

Document status and date:

Published: 01/01/1996

DOI:

$10.26481 / \mathrm{dis} .19960531 \mathrm{pb}$

Document Version:

Publisher's PDF, also known as Version of record

\section{Please check the document version of this publication:}

- A submitted manuscript is the version of the article upon submission and before peer-review. There can be important differences between the submitted version and the official published version of record.

People interested in the research are advised to contact the author for the final version of the publication, or visit the DOI to the publisher's website.

- The final author version and the galley proof are versions of the publication after peer review.

- The final published version features the final layout of the paper including the volume, issue and page numbers.

Link to publication

\footnotetext{
General rights rights.

- You may freely distribute the URL identifying the publication in the public portal. please follow below link for the End User Agreement:

www.umlib.nl/taverne-license

Take down policy

If you believe that this document breaches copyright please contact us at:

repository@maastrichtuniversity.nl

providing details and we will investigate your claim.
}

Copyright and moral rights for the publications made accessible in the public portal are retained by the authors and/or other copyright owners and it is a condition of accessing publications that users recognise and abide by the legal requirements associated with these

- Users may download and print one copy of any publication from the public portal for the purpose of private study or research.

- You may not further distribute the material or use it for any profit-making activity or commercial gain

If the publication is distributed under the terms of Article $25 \mathrm{fa}$ of the Dutch Copyright Act, indicated by the "Taverne" license above, 


\section{Non-invasive methods for the assessment of wall shear rate and arterial impedance}




\section{Non-invasive methods for the assessment of wall shear rate and arterial impedance}

\section{Proefschrift}

ter verkrijging van de graad van doctor aan de Rijksuniversiteit Limburg te Maastricht, op gezag van de Rector Magnificus, Prof. mr. M.J. Cohen, volgens het besluit van het College van Dekanen, in het openbaar te verdedigen op vrijdag 31 mei 1996 om 16.00 uur

door

Peter Jacobus Brands

Geboren te Steenbergen op 22 maart 1959 
Promotores: $\quad$ Prof.Dr.Ir. A.P.G. Hoeks

Prof.Dr. R.S. Reneman

Beoordelingscommissie: Prof.Dr. H.A.J. Stuijker Boudier (voorzitter)

Prof.Dr.Ir. N. Bom (Erasmus Universiteit Rotterdam)

Prof.Dr.Ir. A. Hasman

Prof.Dr. P.J.E.H.M. Kitslaar

Prof.Dr. N. Westerhof (Vrije Universiteit Amsterdam)

Het verschijnen van dit proefschrift werd mede mogelijk gemaakt door

financiële steun van:

-Pie Medical Benelux BV

-Nederlandse Hartstichting

ISBN 90-5681-(005-7 
Aan Mireille, Amadeo en Sam 


\section{Contents}

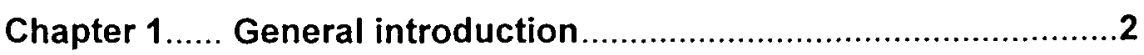

Chapter 2..... Principles of mean frequency estimators for ...........19 ultrasound signals

Chapter 3..... Discrimination filters for the different. ultrasound signal components

Chapter 4..... The effect of discrimination filters on the mean .57 blood flow velocity estimation range of the RF cross-correlation model (CCM) estimator

Chapter 5..... A non-invasive method to estimate wall shear .77 rate using ultrasound

Chapter 6..... Validation of the method to estimate wall 93 shear rate

Chapter 7..... A non-invasive method to estimate dimensionless.111 arterial impedance by means of ultrasound

Chapter 8 ..... In vivo validation of the assessment of dimensionless arterial impedance

Chapter 9..... General discussion and conclusions.

References 147

Summary .156

Samenvatting .159

Nawoord. 162

Curriculum Vitae 164 


\section{Chapter 1}

\section{General introduction}

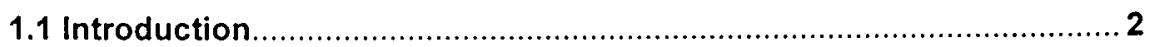

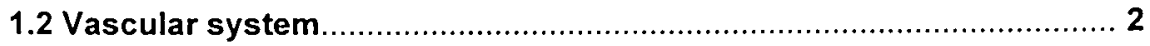

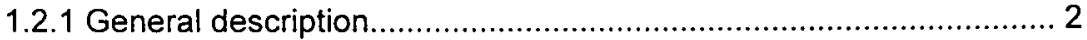

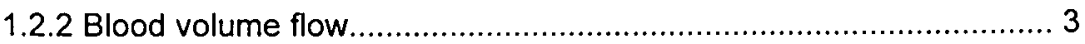

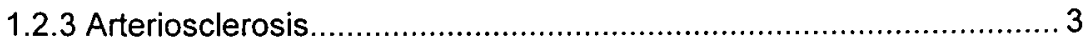

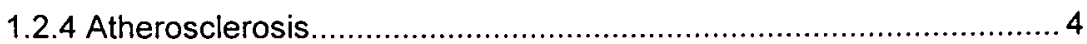

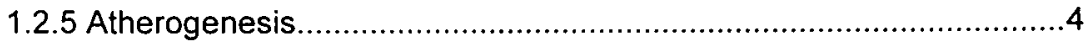

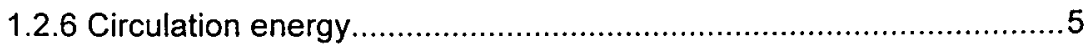

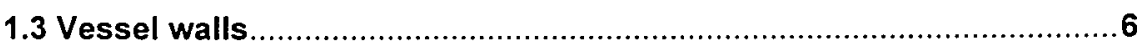

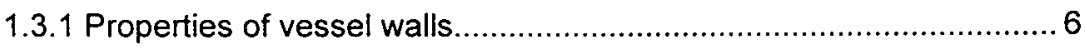

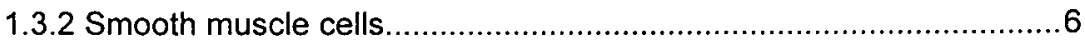

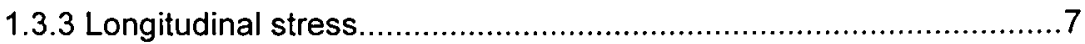

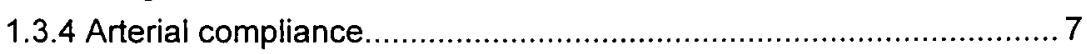

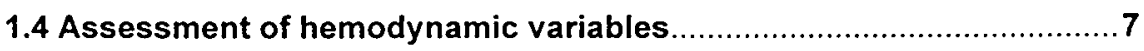

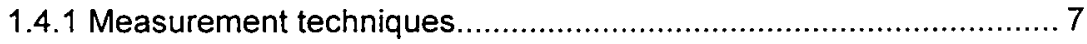

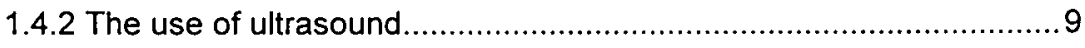

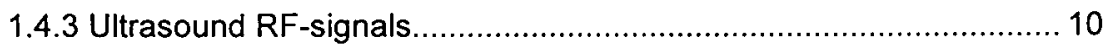

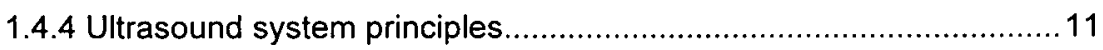

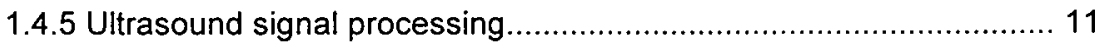

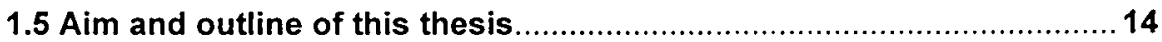

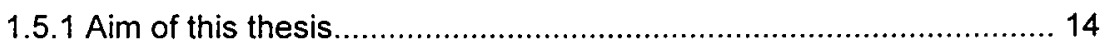

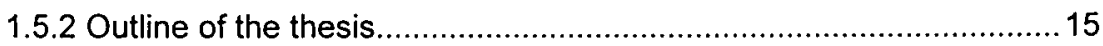




\subsection{Introduction}

In this thesis methods are developed for the non-invasive assessment of wall shear rate and arterial impedance in arteries by means of ultrasound. Wall shear rate is the near wall velocity gradient with respect to the radius. This general introduction starts with a brief description of the vascular system, properties of the blood vessel wall, and the employment of ultrasound for the assessment of hemodynamic variables. The interrelationship between vascular hemodynamic parameters is used to study the properties of the vascular system. For example, wall shear rate describes the relationship between the diameter of a blood vessel and the blood volume flow, while arterial impedance describes the relationship between the pressure and volume flow waveforms. The methods developed for the assessment of wall shear rate and arterial impedance are based on dedicated signal processing of received ultrasound waves. The principles of this signal processing are briefly described and the chapter concludes with a presentation of the aim and outline of this thesis.

\subsection{Vascular system}

\subsubsection{General description}

The immense network of blood vessels of the systemic circulation can be classified in an arterial and a venous system. The heart, a hollow thick-walled muscle being the pump of the vascular system, is pumping rhythmically blood into the aorta resulting in a pulsatile pressure and blood volume flow in the arterial network. The main objective of the blood volume flow circulation is the transportation of oxygen and nutritives to the cells and waste products from the cells in order to keep the organism alive. Other objectives are temperature regulation and hormone transportation. The aorta and the arteries nearest to the heart are elastic and act as a temporary buffer for the blood volume ejected from the left ventricle into these vessels in the systolic phase of a heart cycle (windkessel effect) (Hales 1733). The elastic properties of the arteries have a cushioning effect, i.e., smoothing the pressure and flow pulses. At the end of the arterial system, at the level of the capillaries, the perfusion pressure is far more stable and the volume flow is far more stationary. The venous system acts as a blood-volume reservoir for the rhythmically pumping heart. Therefore, the volume of blood is much larger in the venous part than in the arterial part of the circulation. The veins have also elastic properties but with a different objective than the arteries. The elastic behaviour of the veins allow them to expand or constrict (volume increase or decrease) with the function to redistribute blood from the venous to the arterial part of the circulation. An artery contains a distinct elastic layer giving it its compliant property, whereas a vein wall mainly consists of connective tissue with smooth muscle cells for the blood-volume redistribution. 


\subsubsection{Blood volume flow}

Blood is a non-Newtonian fluid and extremely well fit for its function. Blood combines a high volume fraction of red blood cells $(45 \%)$ with a low viscosity due to the deformability of these cells. The high concentration of red blood cells makes the blood flow highly dependant on the mechanical properties of these cells. Therefore, distribution of the red blood cells and platelets over the cross-section of the vessel is fully controlled by the flow of the red blood cells in the vessel. The most obvious feature of blood volume flow in arteries is its pulsatility. This pulsatility is possibly based on the principle of optimal adaptation between form, function and energy load to the heart (Rodbard 1970, Rodbard 1975). An other example of this principle could be the fact that the red blood cells are the only cells without a nucleus, this to reduce mass to facilitate transportation. The fluid mechanical forces involved in blood volume flow, are in subtle balance between unsteady inertia, convective inertia, pressure and viscous forces (Womersley 1957). Pressure is the driving force per area, while inertia and viscous forces form the opposition to volume flow. It is believed that there is an important relationship between atherosclerosis, the characteristics of blood volume flow and mechanical forces in the arteries (Friedman 1990, Nerem and Girard 1990, Nerem 1992).

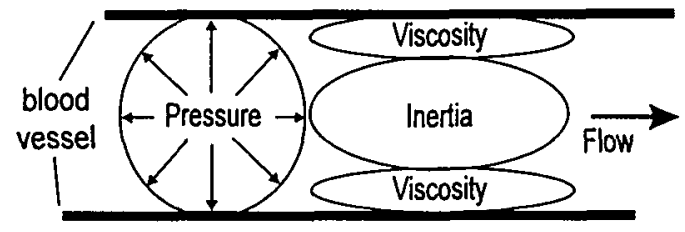

Figure 1.1: Schematic presentation of the fluid mechanical forces: pressure is the driving force while viscosity and inertia form the opposition to volume flow.

The blood flow velocity distribution over the cross-section of the blood vessel (i.e. the velocity profile) as function of time can provide information about the mechanical forces acting in these arteries.

\subsubsection{Arteriosclerosis}

Arteriosclerosis is the structural changes in arterial walls due to ageing. The changes with age in the systemic arteries of humans are mainly dilation and stiffening of the vessels associated with thickening of their walls. Wall properties changes related to age do not just develop in the elderly but are progressive throughout life (Reneman et al. 1986). These arterial changes are generally attributed to fatiguing effects of cyclic stress. Cyclic stress "fatigues" any material by reorganising its crystalline structure and causing it to fracture at a level of stress it was previously able to bear. There are no reasons to conceive that elastin and collagen fibres in the arterial vessel wall can be immune for this process. On the contrary, it is reasonable to assume that the artery is 
adapting to fatiguing effects by a progressive dilation, stiffening of the vessel and thickening of the vessel wall, resulting in the transfer of stress from the elastin to collagen fibres.

\subsubsection{Atherosclerosis}

Atherosclerosis is a disease, not a normal ageing process, and is possibly related to a life style. Atherosclerosis has its major ill-effects through restricting mean blood volume flow to particular organs such as heart, brain, kidney, and eye.

\subsubsection{Atherogenesis}

Atherogenesis the process that may lead to atherosclerosis, starts possibly at birth, and is characterized by a time span in the order of decades. There have been many excellent studies about this "disease" process and its evolution (Schwartz and Mitchell 1972, Ross and Glomset 1976, Ross 1981, Ross 1990, Schwartz et al. 1991, Nerem 1992). However, these studies do not tell us about cause and effect but only over the various risk factors and the relation of the "disease" with life style. The relation between atherosclerosis and fluid dynamics has been prospected (Caro 1977, Zarins et al. 1987). From in vivo studies, there is evidence of hemodynamic influences on atherogenesis by means of the effect on the endothelial cells and on intimal thickening (Friedman et al. 1981, Friedman et al. 1986, Friedman et al. 1987). Furthermore, in vitro studies have shown that endothelial cells undergo a variety of structural and functional changes in response to the frictional drag (wall shear stress or shear rate) of an imposed fluid volume flow (Davies et al. 1983, Davies 1988, Davies and Barbee 1994).

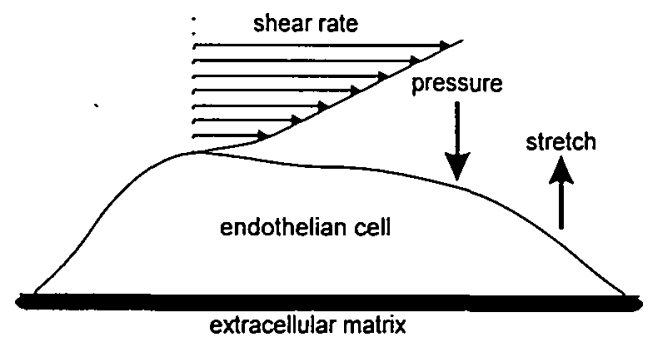

Figure 1.2: Mechanical stimuli acting on endothelial cells.

Wall shear stress describes the relation between the viscosity $\eta[P a . s]$ of the fluid (blood), the radius $R$ of the tube (blood vessel) and the flow velocity $v$ of the fluid (blood) as $\tau=\eta, \partial v /\left.\partial r\right|_{\mathrm{r}=\mathrm{R}}[\mathrm{Pa}]$. The derivative of the velocity $v$ with respect to the radius $r$ is defined as shear rate $\gamma=\partial v / \partial r[1 / s]$. Rodbard has examined the thesis that the cells of the cardiovascular system have sensors for specific mechanical stresses (Rodbard 1970, Rodbard 1975). Kamiya observed that the wall shear stress is of the same order of magnitude $(1.5 \mathrm{~Pa})$ in all generations of arteries, large and small, including the aorta and 
capillaries (Kamiya and Togawa 1980, Kamiya et al. 1984). This observation supports the concept that there is an optimal shear stress in the arterial system and a regulation, by means of diameter changes, to the optimal shear stress of $1.5 \mathrm{~Pa}$. A better knowledge of the relation between shear rate or stress abnormalities and vessel wall properties presumably helps to improve our insights into the process of atherogenesis.

\subsubsection{Circulation energy}

It is obvious that the functional properties and geometry of the vascular system are evolved so that the transportation of oxygen and nutritive substances to the cells and waste products from the cells is performed with a minimum of energy consumption to keep the load for the heart as low as possible. The principle of optimality in biology was proposed by Rashevsky, implying that evolution leaves most biological systems in an optimally designed form (Rashevsky 1960). One of the most obvious features demonstrating this principle is the rhythmical pumping of the heart in combination with the elastic properties of the arterial wall. The buffer capacity $C$ of the elastic arterial vessels takes care of the temporary storage of the systolic volume flow pulse reducing the energy consumption of the heart (heart load). This property is shown in figure (1.3).

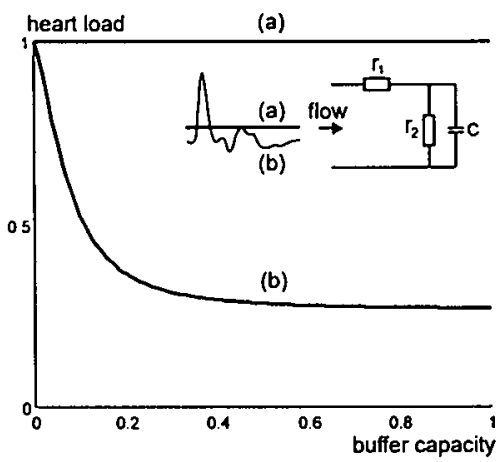

Figure 1.3: The normalized heart load as function of normalized arterial buffer capacity (compliance) for a stationary volume flow (a) and a pulsatile volume flow (b). $r_{l}$ : inflow resistance; $r_{2}$ : outflow resistance; $C$ : buffer capacity (compliance)

In the situation of a stationary volume flow (Fig 1.3a) the heart load is independent of the buffer capacity and determined by the inflow and outflow resistance. In the situation of a pulsatile volume flow (Fig. 1.3b) the heart load is a function of the buffer capacity (temporary storage of the systolic volume flow pulse) and determined by the inflow resistance, the outflow resistance and the buffer capacity (complex impedance). This model demonstrates that under pulsatile volume flow conditions the elasticity of the artery wall reduces heart load. However, it should be noted that the difference in heart load for the stationary and pulsatile volume flow condition is not representative for the real situation because of the simplification of arterial impedance in the model. 
Arterial impedance describes the relationship between the pressure and volume flow waveforms in an artery and characterizes the properties of the vascular bed downstream. Arterial impedance is completely independent of the volume flow wave into the arterial system and so determines the amplitude and contour of the pressure waveform generated by any volume flow waveform into the vascular bed. This independency makes input impedance of particular value in the studies on heart load and vascular circulation (O'Rourke and Taylor 1967, Westerhof and Noordergraaf 1970, Westerhof and Elzinga 1974, Milnor 1975, O'Rourke et al. 1984).

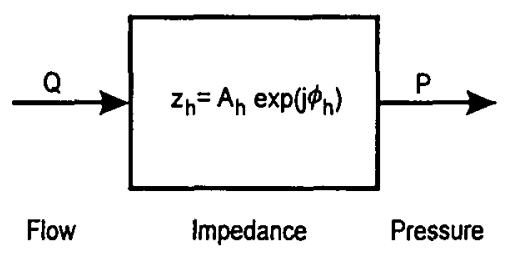

Figure 1.4: Schematic diagram of the relationship between volume flow, impedance and pressure ( $h$ represents the corresponding harmonic components).

\subsection{Vessel walls}

\subsubsection{Properties of vessel walls}

About 70 percent of the wall of arteries and veins consists of water, which is not elastic but has the ability to withstand compression. The rest of the wall material consists of a mesh of fibres which do have elastic properties. There are two sorts of fibres: elastin and collagen. They are fibrous in nature and the collagen fibres are less elastic than the elastin fibres. In large arteries elastin and collagen together constitute 50 percent of the dry mass. Another major component of the arterial wall is smooth muscle which, while contributing to the tension in the wall, cannot properly be regarded as a true elastic material. The arterial wall is divided into three concentric layers: the intima, media and adventitia. The intima consists of the vascular endothelium, which is a single layer of cells. Demarcation between the intima and media is provided by the internal elastic lamina. The adventitia is a layer of collagen and some elastin tissues that merges with the surrounding connective tissue. The media forms the largest part of the wall and characterises the vessel's mechanical properties.

\subsubsection{Smooth muscle cells}

Smooth muscle cells are the active components in the vascular wall. These cells have a circumferential orientation and their contraction is mainly in this direction. It has become apparent that the endothelium plays a major role in the modulation of vascular smooth muscle tone (contraction and relaxation) (Ryan et al. 1979, Furchgott and 
Zawadzki 1980). The contractile stress of vascular smooth muscle is capable of decreasing the diameter of even the largest arteries.

\subsubsection{Longitudinal stress}

Arteries in the body are under a condition of longitudinal stress and the magnitude is at most a factor 1.2 higher than the zero-stress state (Deng et al. 1994). The cause of longitudinal stress is still a matter of debate. It has been postulated that it results from differences in growth rates between the artery and the surrounding tissues. The longitudinal stress of the pulmonary artery has a rhythmical component due to breathing. Smith postulated that this could be a factor involved in elastin fragmentation (Smith 1976, Pevec et al. 1993).

\subsubsection{Arterial compliance}

Compliance $C$ describes the change in vessel lumen volume $V$ for a given increase in stress $p$, i.e. $C=d V / d p\left[\mathrm{~m}^{3} / \mathrm{Pa}\right]$. The structural composition of the arterial media determines the compliance of the artery (O'Rourke 1982). As the arterial media of the muscular arteries ,e.g. femoral artery, is composed of both smooth muscle cells and connective tissue the pressure volume relationship is non-linear ( $\mathrm{O}^{\prime}$ Rourke and Avolio 1980). When the distending pressure is low the tension is borne by elastin fibres and the compliance is high. When the distending pressure is high, however, the tension is borne predominantly by the less extendible collagen fibres and the arterial wall is less compliant. Compliance depends partly on blood pressure, but importantly more on the elastic properties and composition of the arterial wall. With some acceptable simplifications (no longitudinal strain) compliance can be expressed as $C=d A / d p$ $\left[\mathrm{m}^{2} / \mathrm{Pa}\right]$ with $d A$ as the local cross-sectional artery area change (Reneman et al. 1986). The latter can be calculated from diameter $D$ and distension $\Delta d$ which can be determined non-invasively and very accurately with the use of ultrasound (Hoeks et al. 1985, Hoeks et al. 1990) assuming the cross-section to be circular. The non-invasive assessment of local pulse pressure is a problem but for some arteries (e.g. carotid artery) an estimate of compliance can be obtained with the pulse pressure recorded in the brachial artery as a substitute for local pulse pressure (Reneman et al. 1992). Most of the studies about compliance are achieved in this way (van Merode et al. 1988, Arcaro et al. 1991, LaColley et al. 1992, Benetos et al. 1993, Hansen et al. 1993, Sonesson et al. 1993, Girerd et al. 1994, Laurent et al. 1994).

\subsection{Assessment of hemodynamic variables}

\subsubsection{Measurement techniques}

The assessment of hemodynamic variables such as pressure, blood volume flow, blood flow velocity, blood flow velocity distribution, vessel wall distension, vessel wall thickness, arterial impedance, blood viscosity, shear rate and pulse wave velocity is 
important. First of all, to get indices of cardiovascular well-being. Second, to find characteristic hemodynamic parameters. Third, to obtain information about the effect of therapeutic interventions. In the past century there has been an evolution in the development of techniques to measure the above mentioned variables. Each hemodynamic variable needs its specific transducer and the technology to process the information (data stream) from the transducer. Research for different transducers started already in the 18th century (Hales 1733). For example, the development of a reliable pressure transducer (manometer) was initiated by Otto Frank (Frank 1903). This type of transducer was used by Wiggers to study the pulse pressure in the cardiovascular system (Wiggers 1928). The indirect measurement of blood pressure is obtained by means of the auditory detection of the pulse distal to an occlusive cufl. This principle is used in the ultrasonic determination of blood pressure were an ultrasound transducer detects the motion of the blood vessel under an occlusive cuff (Stegall et al. 1968).

Many of the hemodynamic variables are inaccessible because the proper transducer cannot be used non-invasively, the technology for the data processing is not available, or the energy applied to the tissue to extract information from inside the body is not harmless. The idea to use backscattered energy as an information source for processes in the human body originated in the beginning of the $19^{\text {th }}$ century. The practical use of this idea for the assessment of hemodynamic variables had to wait for developments in signal processing and electronics. The development of signal processing and electronics for radar applications was pushed in the $1940^{\mathrm{s}}$ (Rabiner and Gold 1975). It were these developments which initiated the use of ultrasound for the assessment of hemodynamic variables. The signal processing needed for radar, to assess location and velocity, is almost identical to the signal processing needed for ultrasound systems. Already in the $1950^{\mathrm{s}}$ the first ultrasound systems for the assessment of blood flow velocity became available (Kalmus 1954, Franklin et al. 1959, Kaneko et al. 1966). In the past decades these systems were gradually improved (Woodcock 1975). Further developments of these systems are still on their way. The assessment of blood flow velocity with ultrasound has become very popular since the $1970^{\mathrm{s}}$ (Reneman 1974, Webster 1978) because of its non-invasive and harmless character (Wells 1974). The number of articles about blood flow velocity assessment with ultrasound became almost uncountable. Nowadays ultrasound can be used to obtain hemodynamic variables as vessel diameter, vessel wall distension, (Lindstrom et al. 1977, Hoeks et al. 1985, Hoeks et al. 1990, Hayoz et al. 1992), vessel wall thickness (Farrar et al. 1978, Pignoli et al. 1986, Tardy et al. 1992), arterial impedance (Powalowski 1989), velocity waveforms, velocity profiles (Keller et al. 1976, Hoeks et al. 1981, Reneman et al. 1985, Reneman et al. 1986), shear rate (Brands et al. 1995) and pulse wave velocity (Lehmann et al. 1994). Another promising non-invasive technique to assess blood volume flow and three dimensional blood flow velocity distributions is magnetic resonance imaging (MRI) (Dumoulin et al. 1993). Assessment of hemodynamic variables is not an objective in itself but should be used to study the interrelationship between the different variables in order to define hemodynamic characteristics. The definition of hemodynamic 
characteristics is very complicated because of the inherent variability among humans and the variability in the measurement techniques.

\subsubsection{The use of ultrasound}

Nearly all non-invasive biomedical measurements depend on some energy being applied to the tissue, e.g., X-ray (Rontgen 1895), electromagnetic fields or ultrasound. It is obvious that the energy levels of the various types of energy should be harmless at the time of assessment as well as afterwards. Ultrasound is a high frequent $(2-10 \mathrm{MHz})$ shock wave that can be used non-invasively and which is at the diagnostic level harmless for the tissue (Wells 1974). With a piezoelectric crystal an electric signal is converted to an uitrasound shock wave. This can be a pulse or a continuous wave. The dimensions of the ultrasound field, caused by the ultrasound wave, depend on the properties of the piezoelectric crystal and on the tissue to which the ultrasound wave is applied. The properties and dimensions of the ultrasound field are very complicated and have been subject of many studies to create mathematical models (Chivers and Santosa 1986, Jongen et al. 1986, Cassereau and Guyomar 1988, Linssen and Hoeks 1990). At transitions in acoustic impedance, caused by interfaces of structures, the ultrasound waves will be partly reflected. The travelling ultrasound waves are attenuated due to absorption and scattering. The absorption is proportional to the square of the emission frequency used. Thus the higher the ultrasound frequency is, the higher the attenuation will be. Because the depth of the vessel of interest is anatomically determined, attenuation can only be influenced by selecting properly the emission frequency. Consequently, ultrasound signals returned from a greater depth have a lower signal to noise ratio (SNR) than signals from nearby. The change to a lower emission frequency to measure at a greater depth with an acceptable SNR has the disadvantage of loss of resolution.

The ultrasound waves returned from inside the body may have two carriers of information: reflection and scattering. If the dimension of the acoustic boundary is greater than the wavelength of the ultrasound wave transmitted the sound wave will be partly reflected. Reflection will take place under the same angle as the angle of incidence. However, if the dimension of the acoustic boundary becomes small as compared to the wavelength of the ultrasound wave transmitted, the sound wave will be scattered in all directions. For example, in a blood vessel scattering will take place because the dimensions of a red blood cell $(\varnothing 8 \mu \mathrm{m})$ and the inter spacing between them is much smaller than the ultrasound wavelength, e.g., $6 \mathrm{MHz}(\lambda=250 \mu \mathrm{m})$. However, if the inter spacing becomes small as compared to the dimension of a red blood cell these cells can form a cluster which is greater than the wavelength of the ultrasound wave and the sound wave will be partly reflected on such a cluster. It is obvious that the reflected power is greater than the backscattered power. An increase of the emission frequency of the transmitted ultrasound wave will increase the backscattered power because then the dimension of the scatterers relative to the wavelength will become larger. In a theoretical study Angelsen described the 
complicated scattering behaviour of ultrasound from blood (Angelsen 1980). Concluding that if the power of the backscattered ultrasound wave has a Raleigh distribution, this power is proportional to the fourth power of the emission frequency. On the other hand the absorption, as already mentioned, is proportional to the square of the emission frequency. Therefore, the optimum emission frequency as function of the backscattered power from the red blood cells, is a function of depth and emission frequency (Baker et al. 1978).

\subsubsection{Ultrasound RF-signals}

$\mathrm{RF}$-signals are electric radio frequent signals (RF-signals) transformed from the received ultrasound shock waves. This transformation, from ultrasound shock waves to electric RF-signal, takes place in the piezoelectric crystal. The ultrasound RF-signal has a center frequency ranging from 2 to $10 \mathrm{MHz}$ and a quality factor $\left(Q=f_{c} / B\right.$ the ratio between center frequency $f_{c}$ and bandwidth $B$ ), depending on the properties of the piezoelectric crystal and is normally in the range from 2 to 8 . The carriers of information in a single RF-signal consist of reflection and scattering. These information carriers are mixed with each other, with noise and with reverberations. The latter are reflected ultrasound waves from acoustic boundaries in between (secondary reflections). These secondary reflections may manifest itself in the lumen of a blood vessel or at places with almost no reflections. The power of the reflections is of the order of $40 \mathrm{~dB}$ greater than the power of the scattering while the power of the reverberations is of the order of $20 \mathrm{~dB}$ greater than the power of the scattering. The difference in power between reflection and scattering heavily depends on the number of scattering particles, the depth, the center frequency and the power of the ultrasound pulse.

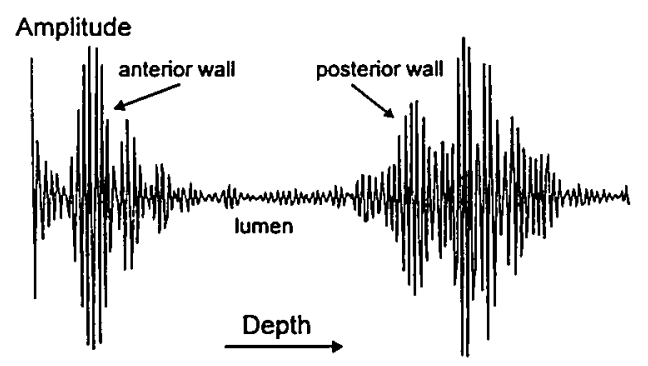

Figure 1.5: An example of an RF-signal as received from the intersection of the ultrasound beam and a blood vessel.

It should be noted that the center frequency of the RF-signals is a function of depth due to the frequency dependent attenuation of the ultrasound wave in the tissue. Highfrequent ultrasound waves are more attenuated than low-frequent ultrasound waves This attenuation effect will result in a down shift of the center frequency as function of depth. 


\subsubsection{Ultrasound system principles}

In ultrasound systems the sound waves can be emitted continuously (CW) or as a pulse (PW). CW systems are mainly used for the assessment of blood flow velocity. PW systems transmit periodically a burst of ultrasound and are used for the assessment of blood flow velocity and its distribution as well as for the visualisation of internal structures because the pulsed nature adds the possibility of measuring distance. CW systems do not provide depth information. CW systems use two crystals: one for the transmission and one for the reception of ultrasound waves, while PW systems may use only one single crystal for both transmission and reception. The resolution in depth of the CW system depends on the volume bound by the overlap of the transmitted and received ultrasound waves. This volume is a function of the properties and the angle of the two crystals. CW systems can use a low ultrasonic intensity to obtain an adequate SNR, are simple and provide a wide velocity range. In the PW approach the volume from which ultrasound information is received can be selected by means of an electronic range gate, positioned at a certain time delay after transmission of the ultrasound burst (a number of wavelengths). The time delay defines the depth of measurement $\left(2 y=c . t_{\text {delay }}\right.$ roundtrip distance). The shorter the transmitted ultrasound bursts is, the shorter the window of the range gate can be, and the more selective the ultrasound information originating from a particular depth. A multi-gate PW system is a device in which the number of range gates is greater than one (Keller et al. 1976, Brandestini et al. 1979, Hoeks et al. 1981, Reneman et al. 1986). PW systems have a limit as far as the maximally detectable blood flow velocity and the depth of measurement is concerned due to the temporal sample frequency, called the pulse repetition frequency (PRF). The PW approach has the disadvantage that the ultrasonic intensity necessary to obtain an adequate SNR should be high. However, because bursts of ultrasound are used, the temporal mean intensity can still be low. A great advantage of PW systems, over CW systems is the assessment of blood flow velocity profiles by means of the multi-gate approach. The developments in ultrasound systems show a shift from CW systems to PW systems. In the PW systems it self there has been a shift from single gate to multi-gate and from multi-gate to two dimensional blood flow velocity mapping, the so called color flow mapping (Reneman et al. 1986). Color coded ultrasound Doppler systems allow the assessment of blood flow velocity distribution (color encoded) within a plane of observation simultaneously with internal structures (echo image coded in shades of grey).

\subsubsection{Ultrasound signal processing}

Ultrasound signal processing is the extraction of information from the information carriers in the pre-processed RF-signals, where pre-processing depends on the properties of the ultrasound probe, e.g. the quality factor $Q$ and the type of transducer. The carriers of information in the RF-signals received are, as already mentioned, reflection and scattering while the information it self can be divided into two categories: 
1) position and 2) displacement. The relation between reflection, scattering, position and displacement is shown in table (1.1):

Table 1.1: The relation between the information carriers (reflection and scattering) and the information it self (position and displacement).

\begin{tabular}{|c|c|c|}
\hline & reflection & scattering \\
\hline position & structure (tracking) & fluid tracking \\
\hline displacement & structure velocity & fluid velocity \\
\hline
\end{tabular}

Table 1.1 shows that both structural interfaces and small particles can provide information about position and displacement. Ultrasound signal processing is based on spectral analysis or time domain analysis, both with the objective of the estimation of signal variables related to hemodynamic variables. Time domain analysis yields the discrimination between reflections and scattering, and the estimation of signal variables from these discriminated signals within a spatial and temporal estimation window. Frequency domain analysis requires frequency transformation of RF-signals within a spatial and temporal transformation window, providing the estimates of signal variables within this transformation window. Time domain analysis is preferable above frequency domain analysis because the latter needs to compromise between frequency resolution, requiring a long temporal estimation window, and temporal behavior of the observed velocities, requiring a short temporal estimation window. However, frequency domain analysis is very useful for the illustration of the different signal components in the RFsignals (Fig. 1.6). The power spectral density distribution illustrated in Fig. 1.6, may consist of four major components: (1) reflections induced by stationary or slowly moving tissue (high spectral power, narrow bandwidth, low temporal mean frequency); (2) scattering induced by red blood cells (low spectral power, wide bandwidth, temporal mean frequency depending on blood flow velocity); (3) reverberations (low spectral power, narrow bandwidth, low temporal mean frequency); (4) noise (low spectral power, uniform spectral distribution). To estimate blood flow velocity the signal component, induced by reflections or reverberations should be suppressed. The discrimination between reflections and scattering is based on the difference in temporal properties of these signal components (Fig. 1.6).

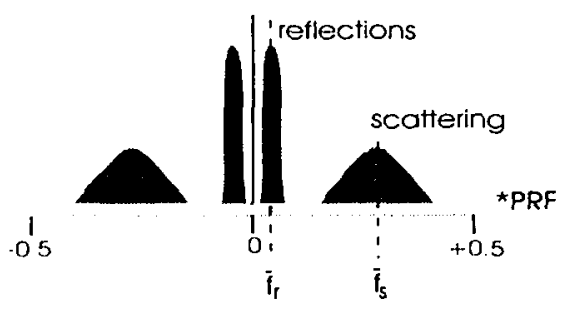

Figure 1.6: The temporal power spectral density distribution of a temporal intersect in a number of RF-signals. 
In general ultrasound time domain signal processing for the assessment of mean blood flow velocity consists of a filter, for the discrimination between reflections and scattering, and a mean frequency estimator for the estimation of the temporal (Doppler frequency) and spatial (carrier frequency) mean frequency which are directly related to the mean blood flow velocity by means of the Doppler equation:

$$
\hat{\overline{\mathrm{v}}}(\mathrm{t}, \mathrm{d})=\frac{\mathrm{c}}{2 \cdot \cos \alpha} \frac{\hat{\overline{\mathrm{f}}}_{\mathrm{t}}(\mathrm{t}, \mathrm{d})}{\hat{\overline{\mathrm{f}}}_{\mathrm{c}}(\mathrm{d})}
$$

where $c$ is the speed of sound in blood, $\hat{\tilde{f}}_{t}(t, d)$ the estimated temporal mean frequency of the scattering at a certain time $t$ and at a depth $d, \hat{\bar{f}}_{c}(d)$ the estimated spatial mean frequency (mean carrier frequency) at depth $d$, and $\alpha$ the angle between the ultrasound beam and the blood velocity direction. The $\cos \alpha$ in this relationship makes it necessary to position the ultrasound beam under an certain angle with the blood vessel. Equation 1.1 is called the Doppler equation because $\hat{\bar{f}}_{t}(t, d)$ can be considered as the Doppler shift in the RF-signals, caused by the velocity of the blood particles. Signal processing can be performed in the RF or in the low frequency domain (LF-domain). In the case of LFdomain processing the RF-signals are demodulated (phase detection) with a reference signal with a constant amplitude and a frequency equal to the frequency of the transmitted ultrasound burst. The mean blood flow velocity is then computed from the estimated temporal mean frequency:

$$
\hat{\overline{\mathrm{v}}}(\mathrm{t}, \mathrm{d})=\frac{\mathrm{c}}{2 \cdot \cos \alpha} \frac{\hat{\overline{\mathrm{f}}}(\mathrm{t}, \mathrm{d})}{\mathrm{f}_{\mathrm{c}}}
$$

where $f_{e}$ is the emission frequency of the transmitted ultrasound burst or wave (reference signal). It should be noted that in the case of $\mathrm{CW}$ ultrasound the depth variable $d$ in $(1.1,1.2)$ is deleted. The difference between RF and LF-domain signal processing is that in the LF-domain the frequency dependent attenuation of the ultrasound burst is neglected by the processing procedure. In the RF-domain it is not necessary to neglect the frequency dependent attenuation because than it is a matter of employing a mean frequency estimator which is capable of estimating the temporal as well as the spatial mean frequency. A consequence of neglecting the frequency dependent attenuation is that the velocity at greater depths is underestimated (1.1).

In the past decades there has been a continuous evolution in the development of discrimination filters and mean frequency estimators for the LF- as well as for the RFdomain. Miller used a covariance approach to spectral moment estimation (Miller and Rochwarger 1972), Brandestini developed a multi-gate pulsed Doppler system (Brandestini et al. 1979), Hoeks developed such a system with serial data processing in combination with a vessel wall movement detector (Hoeks et al. 1981), Angelsen used discrete time estimation of the mean Doppler frequency (Angelsen and Kristoffersen 
1983), Namekawa developed a blood flow imaging system with a auto-correlation mean frequency estimator (Namekawa et al. 1982), Azaria used RF cross-correlation for time delay estimation (Azaria and Hertz 1984), Kasai developed a real-time two dimensional blood flow imaging system using an auto-correlation mean frequency estimator (Kasai et al. 1985), Bonnefous developed a time domain formulation of pulsed Doppler ultrasound and blood velocity estimation by cross-correlation (Bonnefous and Pesque 1986), De Jong developed a method for tissue motion by cross-correlation of pulsed ultrasonic echo signals (De Jong et al. 1990), Wilson gave a description of broad-band pulsed Doppler ultrasound processing using the two dimensional Fourier transformation (Wilson 1991), Ferrara developed a wideband spread target maximum likelihood estimator (Ferrara and Algazi 1991), Brands developed a comparison method for mean frequency estimators (Brands and Hoeks 1992), Hoeks developed an efficient algorithm to eliminate low frequency Doppler signals (Hoeks et al. 1991), Jensen investigated the effect of stationary echo cancelling in velocity estimation by time-domain crosscorrelation (Jensen 1993) and Hoeks developed a processing scheme for velocity estimation using an ultrasound RF cross-correlation model (Hoeks et al. 1994). The main objective in this development over more than twenty years was the selectivity of the discrimination filters and the improvement in quality in mean frequency estimation over short estimation windows.

\subsection{Aim and outline of this thesis}

\subsubsection{Aim of this thesis}

The aim of this thesis was the development and implementation of RF-domain signal processing algorithms for the accurate estimation of blood flow velocity and vessel wall displacement in order to develop methods for the assessment of wall shear rate and arterial impedance. The driving forces behind the development of measurement systems for hemodynamic variables are the questions about the interrelationship between the different variables in vivo.

The mechanical load (shear stress, pressure) and the properties of a blood vessel (diameter, distension, thickness) are dynamically interrelated in a complex way. This interaction is normally in a state of equilibrium. For example, if the blood volume flow through a vessel is structurally increased, the properties of that specific blood vessel are adapted to the new situation and the interaction between the different variables will settle at a new state of equilibrium. The vascular variables involved in this process are: (a) vessel wall diameter in relation to blood volume flow and shear stress, (b) vessel wall thickness in relation to tension (pressure ${ }^{*}$ radius), (c) vessel wall distension in relation to pressure and vessel wall thickness.

Not only the mechanical load plays a role in the geometry and function of the vascular system but also biologic processes like the response of wall shear rate to vasoactive drugs (White et al. 1994). However, these biologic processes are difficult to assess in 
vivo. The hemodynamic variables such as blood flow velocity, diameter and the change in diameter are assessable in vivo with ultrasound. The assessment of shear rate and the study of the interrelationship between the different hemodynamic variables demands a accurate assessment of these variables. This is possible with multi-gate PW ultrasound systems and time domain signal processing (discrimination filter, mean frequency estimator) in the RF-domain.

\subsubsection{Outline of the thesis}

The mathematical principles of estimators are explained in chapter 2. In this chapter attention is paid to: (a) the measures for a "good" estimator in the sense of the quality of the estimate, and (b) the principles of mean frequency estimators as used in ultrasound applications. An ideal estimator should be able to estimate accurately and consistently the concerning variable from a given signal, irrespective of signal properties such as noise, frequency and bandwidth. In practice signal properties the type of estimator as well as the number of sample points used for estimation will affect the quality of the estimate. There are two main classes of estimators: the model-based (or parametric) and model-free (or nonparametric) estimators. The parametric estimator assumes a model with a few characteristic parameters. These characteristic parameters are estimated from a given signal, and can be used as a direct estimate of a physical variable (velocity) or can be substituted in the model to obtain an estimate of the signal based on that model, e.g. regression line. The nonparametric approach does not use a functional model but attempts to estimate a true signal directly from the raw signal (noise reduction with a low pass filter). Most of the mean frequency estimators employed in the ultrasound applications are parametric estimators.

In Chapter 3 the principles of discrimination filters are explained. Attention is paid to: (a) what makes discrimination necessary, and (b) the selectivity of the discrimination. As already mentioned before ultrasound RF-signals are composed of three major signal components, i.e. (Fig. 1.6) reflections, scattering, and noise. These components are mixed in time and depth. The discrimination, based on the difference in temporal properties of the RF-signals, can be obtained with two classes of filters: static filters, having fixed filter characteristics, and dynamic filters, having adaptive filter characteristics. The filter characteristics of a static filter are based on assumptions of signal properties, while dynamic filter characteristics are based on estimates of dynamic signal properties. A great advantage of dynamic filters is their greater capacity to selective discrimination. The two classes of discrimination filters can be applied in both the RF-domain and the LF-domain.

In chapter 4 the effect of discrimination filters on the mean blood flow velocity estimation range of the RF-domain cross-correlation model (CCM) estimator is investigated. Static versus dynamic discrimination filters are compared in performance by means of the available/remaining mean blood flow velocity estimation range. To allow for a direct comparison in performance, as far as the temporal mean frequency 
estimation range is concerned, the different combinations of discrimination filters and $\mathrm{CCM}$ estimator are evaluated for a common simulated RF-matrix (RF-signals in time and depth). It will be demonstrated that dynamic filtration, in combination with the CCM estimator, exhibits the best performance.

In chapter 5 and 6 a dynamic discrimination filter in combination with the RF-domain cross-correlation model (CCM) estimator is presented to be employed for wall shear rate assessment. The internal mechanical forces, acting on a blood vessel are pressure and shear stress. Pressure acts normal to the axis of the wall, while shear stress $\tau$ represents the tangential component of a force field acting on the vessel wall and is defined as:

$$
\tau=\left.\eta \frac{\partial v}{\partial r}\right|_{r=R}
$$

where $\eta$ is fluid viscosity, $\partial v / \partial r$ shear rate, $v$ the axial velocity, $r$ the radial component and $R$ the radius of the blood vessel. The assessment of wall shear rate demands the accurate assessment of near wall blood flow velocity. The validation of the method proposed to estimate wall shear rate was performed through two in vivo and one in vitro study. The first in vivo study concerns a pilot study regarding the reproducibility of the assessment of peak wall shear rate as compared with that of center-line blood flow velocity. The second in vivo study concerns the reproducibility of mean wall shear rate in two subject populations of different age (20-30 years, $n=9 ; 60-70$ years, $n=7$ ). The in vitro study concerns a comparison of wall shear rate assessment obtained with laser Doppler anemometry (LDA) and with pulsed ultrasound in an elastic tube under nonstationary flow conditions (aorta flow pulse).

In chapter 7 and 8 the interrelationship between center-line blood flow velocity and vessel wall distension, used for arterial impedance assessment are discussed. Arterial impedance describes the relationship between pressure and flow and is determined by relating in modules and phase the corresponding harmonics of the pressure and flow signals recorded in an artery:

$$
z_{h}=\left|\frac{P_{h}}{Q_{h}}\right| \exp \left(j \cdot \arg \left(\frac{P_{h}}{Q_{h}}\right)\right)
$$

where $h$ represents the corresponding harmonic component, $z_{h}$ the input impedance, $P_{h}$ and $Q_{h}$ are the complex pressure and flow values for the harmonic component $h$. The heart load can be expressed as the input impedance of the systemic circulation. This features makes input impedance of particular value in the study of heart load and vascular circulation (Murgo et al. 1980). However, the investigation of the relationship between pressure and flow in humans has not been widely applied in medical diagnostics. The reason for this is the inability to measure non-invasively and simultaneously pulsatile pressure and flow from the same location (Mills et al. 1970). 
Investigations of the arterial impedance performed are based on invasive measurements mainly in animals.

The method of non-invasive measurement of the arterial impedance presented in this thesis is based on the normalized interrelationship between distension of the vessel wall and center-line blood flow velocity. For large arteries distension and center-line velocity are almost linear with the change in pressure and flow, respectively. A great advantage of the assessment of arterial impedance by means of the relationship between distension and center-line velocity is that both waveforms can be measured in real-time noninvasively at the same location and simultaneously by means of ultrasound. The method proposed to estimate dimensionless arterial impedance was validated through an in vitro study as well as an in vivo study. The in vivo study concerns a pilot study regarding the reproducibility of the assessment of arterial impedance and the relation with age. The in vitro study concerns a comparison of arterial impedance assessment obtained from the relationship between pressure and flow, and from the relationship between distension and center-line velocity in an elastic tube with known non-stationary flow condition.

A general discussion, conclusions and recommendations for further research concludes this thesis (chapter 9). 



\section{Chapter 2}

\section{Principles of mean frequency estimators for ultrasound signals}

2.1 Introduction

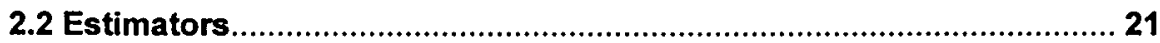

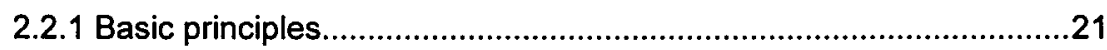

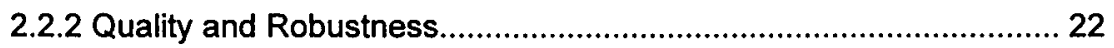

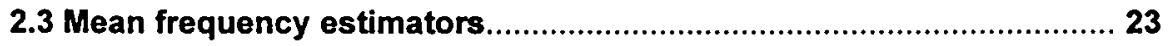

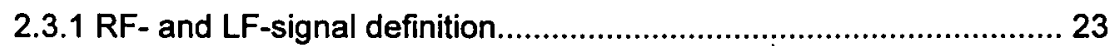

2.3.2 Zero-crossing mean frequency estimator (ZCD)............................... 26

2.3.3 Instantaneous mean frequency estimator (IMF)................................26

2.3.4 Auto-correlation mean frequency estimator (AC)........................... 27

2.3.5 Modified covariance mean frequency estimator (MC) ..................... 28

2.3.6 Complex linear regression mean frequency estimator (CLR).............29

2.3.7 Cross-correlation mean frequency estimator (CC) ......................... 30

2.3.8 Cross-correlation model mean frequency estimator (CCM)...............31

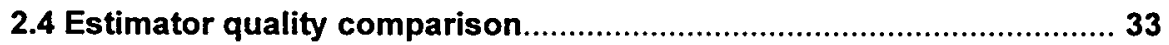

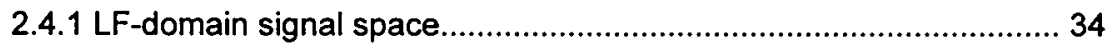

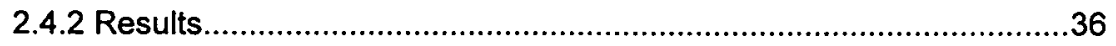

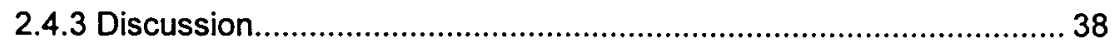

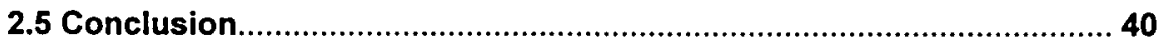

The contents of this chapter has partly been published in the article: Brands P.J., Hoeks A.P.G. A comparison method for mean frequency estimators for Doppler ultrasound. Ultrasonic imaging, Vol. 14, pp. 367-386, 1992 


\subsection{Introduction}

The primary subject of this chapter is to introduce mean frequency estimators as used in uitrasound signal processing and a measure of performance for these estimators.

Mean frequency estimators, as employed in the estimation of mean blood flow velocity (Section 1.4.5), should provide an accurate (quality) and consistent (robustness) estimate of the temporal mean frequency over a wide range of signal conditions and over short estimation windows. Most of the proposed mean frequency estimators are model-based (parametric) estimators. The form of this model is based on prior knowledge and assumptions about the received ultrasound radio frequent signals (RF. signals). To allow for a direct comparison in performance of various mean frequency estimators a method will be described to evaluate the quality and robustness of the mean frequency estimators for a common signal space covering a wide range of realistic parameter combinations. The difference in quality and robustness between the different estimators, under the same signal conditions, can be used as a measure of performance. This method of mean frequency evaluation is illustrated by using the following three different mean frequency estimators: (1) a first order auto-regressive mean frequency estimator; (2) a second order auto-regressive estimator; (3) a complex linear regression estimator. Moreover, the principles of the following seven mean frequency estimators as developed over the past decades will be explained: (1) the zero-crossing mean frequency estimator; (2) the instantaneous mean frequency estimator; (3) the autocorrelation mean frequency estimator; (4) the second order auto-regressive mean frequency estimator; (5) the complex linear regression mean frequency estimator; (6) the RF cross-correlation mean frequency estimator; (7) the RF cross-correlation model mean frequency estimator. The LF-domain mean frequency estimators 1 to 5 and the RF-domain mean frequency estimator 7 are parametric estimators while the RF-domain mean frequency estimator 6 is a non-parametric estimator.

An ideal mean frequency estimator should be able to estimate accurately and consistently the mean frequency of the signal induced by scattering or reflection, irrespective of frequency distribution and the noise level. All the above mentioned factors, as well as the length of spatial and temporal estimation window, will affect the bias and variance of the estimate. For a lower ratio of the scattering and noise power level, or for shorter estimation windows, the variance will be higher. The inherent inconsistency of bias and variance complicates straightforward comparison of different types of mean frequency estimators evaluated under various signal conditions. This drawback may be overcome by introducing measures reflecting the overall behavior of a frequency estimator for a realistic set of signal conditions called the signal space (SP). The signal space is subtended by a set of signal parameters, e.g., amplitude, mean frequency, and bandwidth of the components constituting the LF- or RF-signal, and the length of the estimation window considered. Using computer generated LF- or RFsignals the estimators are evaluated repetitively for each signal condition in the signal space. This evaluation results in an estimate for the bias and variance of the estimate, 
which together constitute the quality $q(m)$ for signal condition $m$. The mean and root mean square (RMS) of $q(m)$ over all signal conditions considered is a measure of the estimator quality $Q_{e s t}$ and robustness $R_{e s t}$, respectively.

The approach outlined above will be demonstrated by comparing three LF-domain mean frequency estimators. They are based on (1) a first and (2) a second order autoregressive model, and (3) a single frequency model using the best fit through the unwrapped phase. The estimators derived from the second order auto-regressive model is able to estimate simultaneously the mean frequency of the reflectors as well as the mean frequency of the scattering (Ahn and Park 1991). Therefore, no preprocessing is required prior to mean frequency cstimation. The other two are single mean frequency estimators and can estimate only one mean frequency in a given estimation window. Thus, to estimate the temporal mean frequency of the scattering with a single mean frequency estimator the reflections should be removed (Chapter 3 ). In the following, a detailed description of the concept of estimators, the different mean frequency estimators as developed in the past decays and the concept of signal space will be given. As to be expected the mean frequency estimators respond differently for a particular signal condition. Therefore, the use of quality $Q_{e s t}$ and robustness $R_{e s t}$ makes it quite simple to appreciate the overall behavior of each estimator.

\subsection{Estimators}

\subsubsection{Basic principles}

An estimator, for a given process parameter, can be derived from a model (parametric estimator) based on prior knowledge of that process, e.g., a power spectral density distribution, or from general assumptions of that process (non parametric estimators), i.e., similarity of signals. If in the case of a parametric estimator the model has a high correlation with the process data, then accurate estimates of the process parameters can be obtained using relatively few samples. On the contrary, non parametric estimators require more samples to obtain accurate estimates, because of the general assumptions made about the process. Thus, models of a process can be employed to reduce the amount of samples required to obtain "good" estimates.

Questions concerning the development of parametric estimators are: What is a "good" model to be used as base for an estimator? How "good" is the resulting estimator? Questions concerning the assumptions of the model are: Is the noise white? Does the random variable have a normal distribution? Is it a stationary process? How many signals are mixed with each other (different sources of data)? Questions concerning the performance of an estimator are: What is the bias of the estimate? What is the variance of the estimate for a given estimation window? It is reasonable to characterize an estimate $\hat{\beta}$ (hat indicates "an estimate of") of the parameter $\beta$ with a given estimator in a given estimation window as being "good" if there is a high probability that the estimate will be close to $\beta$ (low or zero bias, low variance) (Fig. 2.1). 


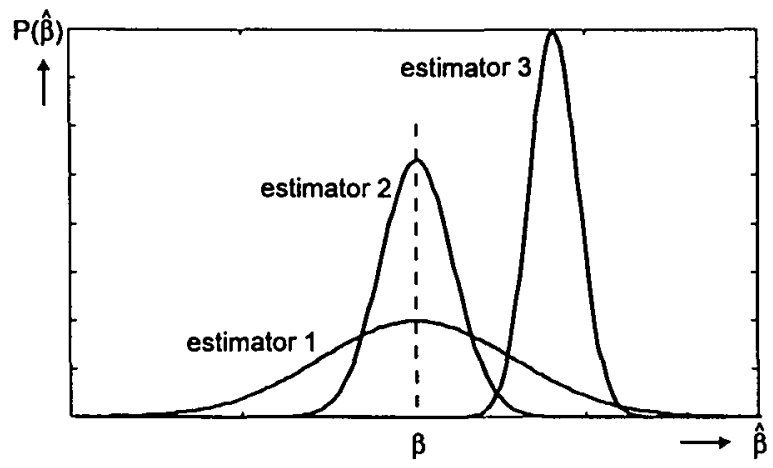

Figure 2.1: Probability density distributions of three estimators for the parameter $\beta$ estimated within the same estimation window.

In Fig. 2.1 estimator 2 is superior to estimator 1 and 3, the variance of estimator 3 is lower than the variance of estimator 1 and 2, where as estimator 1 and 2 have an unbiased estimate while estimator 3 has a biased estimate.

\subsubsection{Quality and Robustness}

Generally, an estimate $\hat{\bar{f}}_{1}(m)$ for the temporal mean frequency $\bar{f}_{1}(m)$ of the through signal condition $m$ simulated scattering will have a bias bias $(m)$ and a variance $\sigma^{2}(m)$ :

$$
\begin{aligned}
& \operatorname{Bias}(\mathrm{m})=\mathrm{E}\left\{\overline{\hat{f}}_{\mathrm{t}}(\mathrm{m})-\hat{\overline{\mathrm{f}}}_{\mathrm{t}}(\mathrm{m})\right\} \\
& \sigma^{2}(\mathrm{~m})=\mathrm{E}\left\{\left(\hat{\overline{\mathrm{f}}}_{\mathrm{t}}(\mathrm{m})-\mathrm{E}\left\{\hat{\overline{\mathrm{f}}}_{\mathrm{t}}(\mathrm{m})\right\}\right)^{2}\right\}
\end{aligned}
$$

where $E$ denotes the expectancy operator. Generally, a quality $q(m)$ of the estimate $\beta$ for signal condition $m$ follows from its mean squared error $M S E$ :

$$
\begin{aligned}
& \text { MSE }=E\left\{(\beta-\hat{\beta})^{2}\right\} \\
& M S E=\sigma_{\hat{\beta}}^{2}+\sigma_{\hat{\beta}}^{2}+b i a s_{\hat{\beta}}^{2}
\end{aligned}
$$

In the case of simulations, the variance of the parameter $\beta$ is zero so the quality $q(m)$ can be defined as:

$$
\mathrm{q}(\mathrm{m})=\sqrt{\sigma_{\hat{\beta}}^{2}(m)+\operatorname{bias}_{\hat{\beta}}^{2}(m)}
$$

The quality $Q_{\text {est }}$ of an estimator in the signal space $S P$ is defined as the mean of $q(m)$ over all signal conditions $m$ considered while the robustness $R_{e s t}$ of an estimator is defined as the root mean square (RMS) of $q(m)$ over all signal conditions $m$ considered. This definition for $Q_{e s t}$ and $R_{e s t}$ means that they are considered in a negative 
development, i.e., a low value of $Q_{e s t}$ and/or $R_{e s t}$ is associated with a "good" quality and/or robustness.

In most cases, it will be problematic or impossible to arrive at an analytical expression for the quality and robustness of the estimate for the temporal mean frequency (Kristoffersen 1988). Hence, one has to resort to experimental evaluation of RF- or LFsignals (Hoeks et al. 1984, van Leeuwen et al. 1986, Hoeks et al. 1993).

\subsection{Mean frequency estimators}

\subsubsection{RF- and LF-signal definition}

The temporal (Doppler frequency) and the spatial (carrier frequency) mean frequency of reflections and scattering in RF- or LF-signals are directly related to the mean blood flow velocity by means of equations (1.1) and (1.2). RF-signals, as already mentioned (Section 1.4.3), are electric radio frequent signals transformed from the received ultrasound shock waves. LF-signals are derived from the RF-signals by means of quadrature demodulation (phase detection) with a reference signal with a constant amplitude and a frequency equal to the frequency of the transmitted burst. A disadvantage of the use of the emission frequency as reference for the quadrature demodulation is that the velocity at greater depths is underestimated due to the fact that the frequency dependent attenuation of the ultrasound burst is neglected.

(c) temporal intersect

(a) RF-matrix

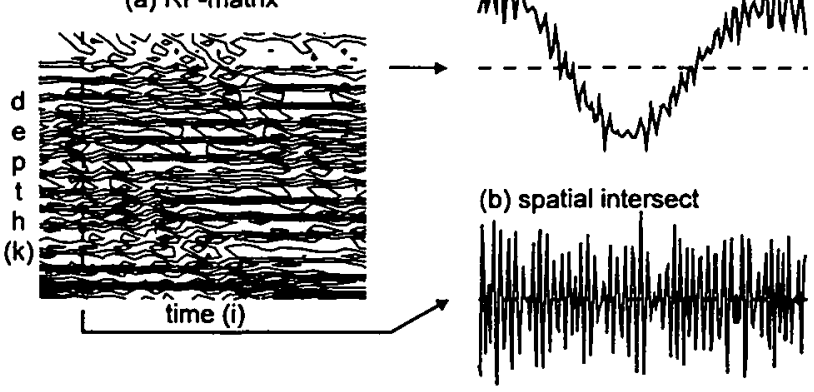

Figure 2.2: The contour map of an RF-matrix (a) together with the signals from a spatial (b) and a temporal (c) intersect.

$\mathrm{RF}$-signals $z_{r}\{k, i)$, where $k$ denotes depth and $i$ denotes time, have a stochastic nature and are sampled in depth with the spatial sample frequency $f_{s-r}$, e.g. three times the emission frequency, and sampled in time with the pulse repetion frequency (PRF):

$$
z_{r}(k, i)=r_{r f}(k, i)+s_{r f}(k, i)+n
$$


where $r_{r f}$ is the reflected signal, $s_{r f}$ the scattered signal and $n$ represents the noise. Because of sampling the frequency range of $z_{r f}$ will extend in spatial direction from $-f_{s-r} / 2$ to $f_{s-r} / 2$ and in temporal direction from $-P R F / 2$ to $P R F / 2$. The RF-samples in time $i$ and depth $k$ form an RF-matrix (Fig. 2.2). In the temporal signal of figure 2.2c the differences in frequency and amplitude between reflections (high amplitude low frequent) and scattering (low amplitude high frequent) are clearly recognisable. In figure 2.3 the relation between the spatial mean frequency $\bar{f}_{c}$, the spatial bandwidth $B$, the temporal mean frequency of the scattered signal $\bar{f}_{s}$ and the temporal bandwidth of the scattered signal $B_{s}$ is shown in a contour map of the two dimensional spectral amplitude distribution of an RF-matrix (Wilson 1991, Wilson and Gill 1993).

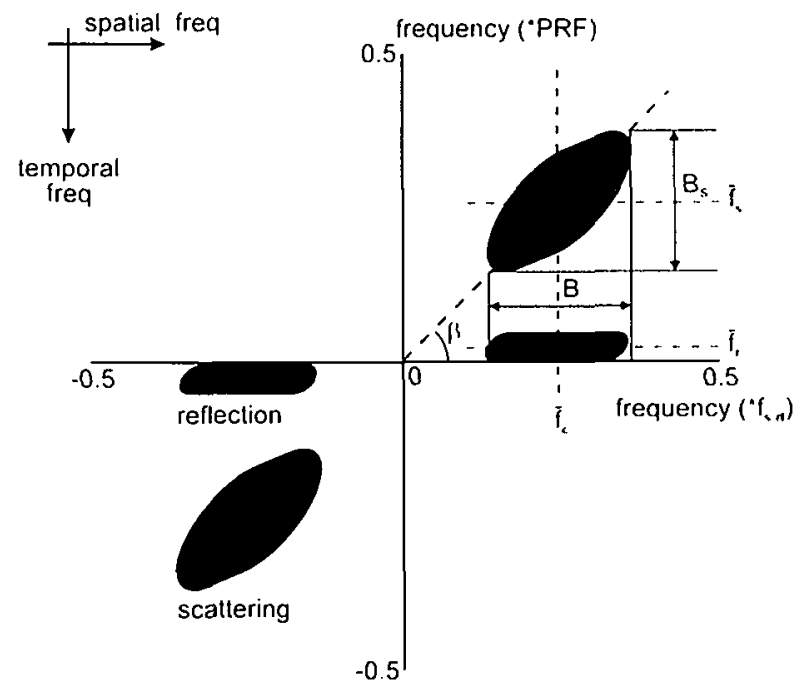

Figure 2.3: Contour map of the two dimensional spectral amplitude distribution of an RFmatrix.

From the bandwidth and the mean frequency relationship of the temporal and spatial signals in figure 2.3 it follows that the temporal quality factor $Q_{\text {t }}$ equals the spatial quality factor $Q$.

$$
\frac{\mathrm{B}_{1} / \mathrm{PRF}}{\mathrm{B} / \mathrm{f}_{\mathrm{s}-\mathrm{rf}}}=\tan \beta=\frac{\overline{\mathrm{f}}_{\mathrm{t}} / \mathrm{PRF}}{\overline{\mathrm{f}}_{\mathrm{c} / \mathrm{f}_{\mathrm{s}-\mathrm{r}}}} \quad \frac{\overline{\mathrm{f}}_{\mathrm{c}}}{\mathrm{B}}=\frac{\overline{\mathrm{f}}_{\mathrm{f}}}{\mathrm{B}_{1}} \quad \mathrm{Q}=\mathrm{Q}_{\mathrm{t}}
$$

where $\beta$ reflects the dimensionless velocity (Section 2.3.8). Relation (2.5) is true for the bandwidth of the reflected signal $B_{r}$ as well as for the bandwidth of the scattered signal $B_{s}$. LF-signals $z_{l}(k, i)$ are sampled in depth with the spatial sample frequency $f_{s-l}$, e.g. the emission frequency divided by the quality factor $Q$ of the ultrasound probe, and sampled in time with the $P R F$ : 


$$
z_{1 f}(k, i)=r_{1 r}(k, i)+s_{1 r}(k, i)+n
$$

where $r_{l f}$ is the reflected signal, $s_{l f}$ the scattered signal and $n$ represents the noise. Because of sampling the frequency range of $z_{l f}$ will extend in spatial direction from $-f_{s-1 /} / 2$ to $f_{s-1 /} / 2$ and in temporal direction from $-P R F / 2$ to $P R F / 2$. The LF-samples in time $i$ and depth $k$ form an LF-matrix (Fig. 2.4).

(a) LF-matrix

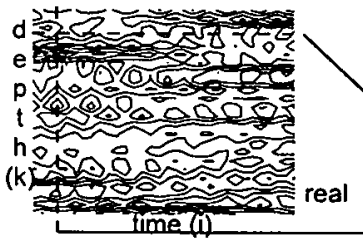

(c) temporal intersect
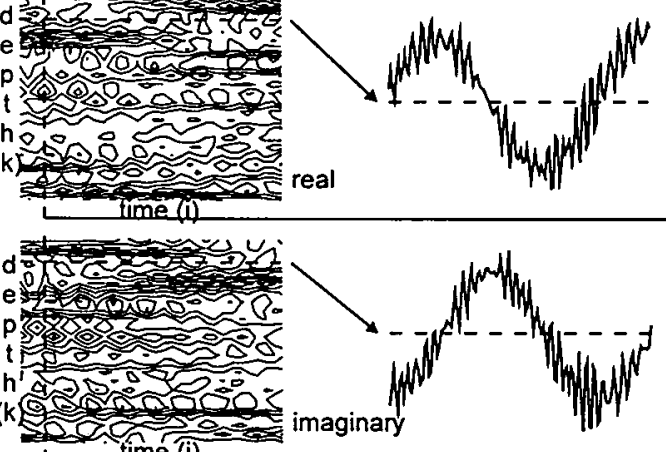

(b) spatial intersect
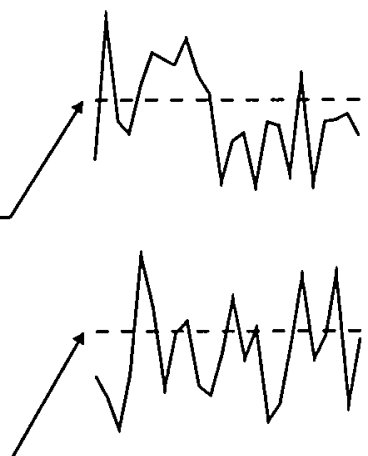

Figure 2.4: The real and imaginary contour map of a complex LF-matrix (a) together with the signals from a spatial (b) and a temporal (c) intersect.

The LF-matrix is complex due to the quadrature demodulation of the RF-signals.

The reflection and/or scattering in the LF- or RF-matrix are subjected to mean frequency estimation in a given estimation window with a length of $N$ and $M$ sample points in temporal and spatial direction, respectively.

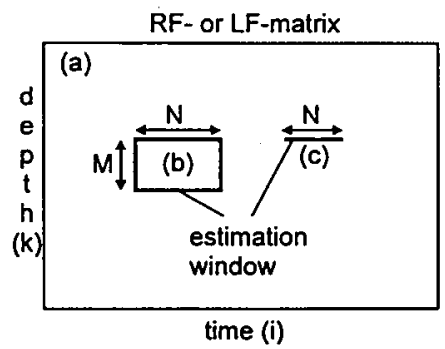

Figure 2.5: An RF- or LF-matrix (a) with the samples $w_{\text {index }}(k, i)$ in a two dimensional estimation window (b) and the samples $w_{\text {index }}\left(k_{a}, i\right)$ in an one dimensional temporal estimation window (c).

To avoid confusion, the variable $w_{\text {index }}(k, i)$, with samples $k$ (depth) $i$ (time) and index lf or $r f$, will be used hereafter to denote the samples in a given estimation window (Fig. 
2.5). The samples $w_{\text {index }}(k, i)$ are, depending on the discrimination filter, samples from scattered signals or reflected signals.

\subsubsection{Zero-crossing mean frequency estimator (ZCD)}

One of the first and widely used mean frequency estimators in Doppler applications in the $70^{\mathrm{s}}$ and even in the $80^{\mathrm{s}}$ was the zero crossing mean frequency estimator (zero crossing detector ZCD) (Reneman and Spencer 1973, Lunt 1975). The ZCD is based on the assumption that the expected value of the zero-crossings per unit of time (length temporal estimation window) in the temporal LF-signal $w_{l f}\left(k_{0, i}\right.$, is a model for the temporal mean frequency of the scattering or reflection:

$$
E\left\{z c\left\{w_{1 f}\left(k_{0}, i\right)\right\}\right\}={ }^{M} \bar{f}_{1} / P R F
$$

where $E$ is the expectancy operator, $z c$ the zerocrossing operator, $={ }^{M}$ denotes "is a model for" and $\bar{f}_{1} / P R F$ the temporal mean frequency as fraction of the $P R F$. From Angelsen and Burckhardt we know that the expected value of zero-crossings per unit of time is certainly not the mean frequency, so the model for the ZCD is based on a false assumption (Angelsen 1980, Burckhardt 1981). Therefore, the ZCD has a biased estimate for high temporal mean frequencies (greater than PRF/4) and for wide band signals (Lunt 1975, Hoeks et al. 1984, van Leeuwen et al. 1986).

\subsubsection{Instantaneous mean frequency estimator (IMF)}

The instantaneous mean frequency estimator (IMF) as proposed by (Angelsen and Kristoffersen 1979) is based on the fact that the averaged first derivative of the phase of a signal with a narrow bandwidth is an estimate of the temporal mean frequency. This narrow bandwidth assumption gives the following model for the temporal LF-signal $w_{l}\left(k_{0}, i\right):$

$$
\text { A.exp }\left\{\text { j. } 2 \pi \text {.i. } f_{1}\left(k_{0}, i\right) / P R F\right\}={ }^{M} w_{18}\left(k_{0}, i\right)
$$

where $A$ is amplitude and $f_{l}\left(k_{0}, i\right)$ the instantaneous frequency (first derivative of the phase) approximated with a difference equation

$$
\frac{f_{t}\left(k_{0}, i\right)}{P R F}=\frac{\left(\phi\left\{w_{1 f}\left(k_{0}, i\right)\right\}-\phi\left\{w_{1 r}\left(k_{0}, i-1\right)\right\}\right)}{2 \pi}
$$

Due to the quadrature demodulation (complex signals) the model (2.8) leads to the following temporal mean frequency estimator:

$$
\frac{\hat{\bar{f}}_{1}}{\operatorname{PRF}}=\frac{1}{2 \pi} \cdot \frac{1}{N} \cdot \sum_{i=1}^{N}\left\{\arg \left(w_{1 r}\left(k_{0}, i\right)\right)-\arg \left(w_{1 r}\left(k_{0}, i-1\right)\right)\right\}
$$


where $\hat{\bar{f}}_{1}$ is the estimated temporal mean frequency of reflection or scattering and arg the operator to compute the phase of a complex sample. The quality of this estimator is comparable with the ZCD (van Leeuwen et al. 1986). The shape of the probability density distribution of the instantaneous frequency, based on sampled observations (2.9) (Hoeks et al. 1984), leads to a high variance and a biased estimated for the high temporal mean frequencies and for wide band signals.

\subsubsection{Auto-correlation mean frequency estimator (AC)}

The auto-correlation mean frequency (AC) estimator as proposed by (Kasai et al. 1985) is based on a first order auto-regressive (AR) model of the temporal LF-signal $w_{l}\left(k_{0}, i\right)$ :

$$
\left\{y(i)=n+a_{1} \cdot y(i-1)\right\}={ }^{M} w_{1 f}\left(k_{0}, i\right)
$$

where $y(i)$ is a complex auto-regressive signal, $n$ Gaussian white noise with zero mean and $a_{1}$ the characteristic parameter of this first order AR model with the following properties (Rabiner and Gold 1975):

$$
\begin{aligned}
a_{1}=p_{1} & p_{1} \text { pole in the unit circle } \\
\arg \left(a_{1}\right)=\bar{\omega} & \text { mean angular frequency of } y(i) \\
\text { 2. }\left(1-\left|a_{1}\right|\right)=b w & \text { bandwidth of } y(i)
\end{aligned}
$$

The auto correlation function of the AR model is obtained as:

$$
\begin{aligned}
R(j) & =E\left\{y(i) \cdot y^{*}(i-j)\right\} \\
& =E\left\{\left(a_{1} \cdot y(i-1)+n(i)\right) \cdot y^{*}(i-j)\right\} \\
& =a_{1} \cdot R(j-1)
\end{aligned}
$$

where $E$ denotes the expectancy operator, $R$ the correlation function and $j$ the correlation lag. From this auto correlation function an estimator for the characteristic parameter $a_{l}$ is derived as:

$$
\hat{a}_{1}=\hat{R}(j) / \hat{R}(j-1)
$$

with:

$$
\hat{R}(j)=\frac{1}{N-j} \sum_{i=j}^{N-1} w_{I f}\left(k_{0}, i\right) \cdot w_{I f}^{*}\left(k_{0}, i-j\right) \quad j \geq 0
$$

Relation (2.12) in combination with (2.14) leads to the following temporal mean frequency estimator: 


$$
\frac{\hat{\overline{\mathrm{f}}}}{\mathrm{PRl}}=\frac{1}{2 \pi} \cdot \arg \{\hat{\mathrm{R}}(1)\}
$$

where $\hat{R}(1)$ is the estimated auto-correlation function with lag 1 and $\hat{\bar{f}}$, is the estimated temporal mean frequency of reflection or scattering. The quality of this estimator is better than the quality of the ZCD and the IMF estimator (van Leeuwen et al. 1986). The improvement in quality is mainly caused by the unbiased estimate of the temporal mean frequency because of the periodic character of the auto-correlation function. An other improvement of this $\mathrm{AC}$ estimator above the $\mathrm{ZCD}$ and the IMF estimators is the unbiased estimate of the mean frequency of partially aliased signals, provided that the temporal mean frequency of the signal is within the temporal frequency range $(-P R F / 2$ to $P R F / 2$ ).

\subsubsection{Modified covariance mean frequency estimator (MC)}

The modified covariance, dual, mean frequency estimator (MC) as proposed by (Ahn and Park 1991) is based on a second order AR model of the temporal LF-signal $w_{l} f\left(k_{0}, i\right)$ :

$$
\left\{y(i)=n+a_{1} \cdot y(i-1)+a_{2} \cdot y(i-2)\right\}={ }^{M} w_{1 r}\left(k_{0}, i\right)
$$

where $y(i)$ is a complex auto-regressive signal, $n$ Gaussian white noise with zero mean and $a_{1}$ and $a_{2}$ the characteristic parameters of this AR model. The characteristic parameters $a_{l}$ and $a_{2}$ are estimated using the modified covariance method (Kay 1988):

$$
\begin{aligned}
& \hat{c}(1,1) \cdot \hat{a}_{1}+\hat{c}(1,2) \cdot \hat{a}_{2}=\hat{c}(1,0) \\
& \hat{c}(2,1) \cdot \hat{a}_{1}+\hat{c}(2,2) \cdot \hat{a}_{2}=\hat{c}(2,0)
\end{aligned}
$$

with the modified covariance $c(j, \tau)$ estimated from $w_{l f}\left(k_{n,}, i\right)$ as:

$$
\begin{array}{rlr}
\hat{c}(j, \tau)=\frac{1}{2(N-2)}\left\{\sum_{i=2}^{N-1} w_{1 f}^{*}\left(k_{0}, i-j\right) \cdot w_{1 f}\left(k_{0}, i-\tau\right)\right. & 1 \leq j \leq 2 \\
\left.+\sum_{i=0}^{N-3} w_{1 f}\left(k_{0}, i+j\right) \cdot w_{11}^{*}\left(k_{0}, i+\tau\right)\right\} & 0 \leq \tau \leq 2
\end{array}
$$

The poles $p_{1}$ and $p_{2}$ in the unit circle are computed from the estimated characteristic parameters $\hat{\mathrm{a}}_{1}$ and $\hat{\mathrm{a}}_{2}(2.18)$ using:

$$
\hat{\mathrm{p}}_{1.2}=\frac{-\hat{\mathrm{a}}_{1} \pm \sqrt{\hat{\mathrm{a}}_{1}^{2}-4 . \hat{\mathrm{a}}_{2}}}{2}
$$

According to the general expression (Rabiner and Gold 1975): 


$$
\begin{array}{ll}
\arg \left(p_{j}\right)=\bar{\omega}_{j} & \text { mean angular frequency } \\
2 .\left(1-\left|p_{j}\right|\right)=b w_{j} & \text { bandwidth of } y(i)
\end{array}
$$

the following temporal mean frequency estimators are derived:

$$
\begin{aligned}
& \frac{\hat{\bar{f}}_{1_{1}}}{\operatorname{PRF}}=\frac{1}{2 \pi} \cdot \arg \left\{\hat{p}_{1}\right\} \\
& \frac{\overline{\bar{f}}}{\text { PRF }}=\frac{1}{2 \pi} \cdot \arg \left\{\hat{p}_{2}\right\}
\end{aligned}
$$

where $\hat{\bar{f}}_{i_{1}}$ is the temporal mean frequency related to estimated pole $\hat{p}_{1}$ and $\hat{\bar{f}}_{i_{z}}$ the temporal mean frequency related to estimated pole $\hat{p}_{2}$. These estimators estimate simultaneously the temporal mean frequency of the reflected signal as well as of the scattered signal. It is reasonable to assume that the lowest absolute temporal mean frequency (either $\hat{\bar{f}}_{i_{1}}$ or $\hat{\bar{f}}_{t_{z}}$ ) belongs to the reflection and the other to the scattering. However, it may be anticipated that the difference in amplitude between reflection and scattering will affect the bias of the estimate. Because of simultaneous estimation of the temporal mean frequency of reflection and scattering, this estimator does not require a discrimination filter prior to mean frequency estimation of either reflection or scattering.

\subsubsection{Complex linear regression mean frequency estimator (CLR)}

The complex linear regression mean frequency estimator (CLR) as proposed by (Brands and Hoeks 1992) is based on an AR model with a single pole on the unit circle in the zdomain:

$$
\left\{y(i)=\exp \left(j \cdot 2 \pi \cdot \text { i. } \tilde{f}_{t} / P R F\right)\right\}={ }^{M} w_{1 r}\left(k_{0}, i\right)
$$

where $\bar{f}_{1}$ is the temporal mean frequency of reflection or scattering. In this AR model the best estimate for $\bar{f}$, in the sense of a minimal mean square error MSE can be derived using:

$$
M S E=E\left\{\left(\left|w_{1 r}\left(k_{0}, i\right)-y(i)\right|\right)^{2}\right\}
$$

Setting the frequency derivative of the MSE equal to zero gives:

$$
\frac{\hat{\bar{f}}}{\text { PRF }}=\frac{1}{2 \pi} \cdot \frac{\sum_{i=-N / 2}^{N / 2} i \cdot \operatorname{uarg}\left\{w_{1 f}\left(k_{0}, i+N / 2\right)\right\}}{\sum_{i=-N / 2}^{N / 2} i^{2}}
$$


where uarg is the operator to unwrap the phase:

$$
\begin{aligned}
& \operatorname{uarg}\left\{\mathrm{w}_{1 \mathrm{fr}}\left(\mathrm{k}_{0}, 0\right)\right\}=\arg \left\{\mathrm{w}_{1 \mathrm{r}}\left(\mathrm{k}_{0}, 0\right)\right\} \\
& \operatorname{uarg}\left\{\mathrm{w}_{1 \mathrm{fr}}\left(\mathrm{k}_{0}, \mathrm{i}\right)\right\}=\operatorname{uarg}\left\{\mathrm{w}_{1 \mathrm{rf}}\left(\mathrm{k}_{0}, \mathrm{i}-1\right)\right\}+\arg \left\{\mathrm{w}_{1 \mathrm{fr}}\left(\mathrm{k}_{0}, \mathrm{i}\right) \cdot \mathrm{w}_{1 \mathrm{rr}}{ }^{*}\left(\mathrm{k}_{0}, \mathrm{i}-1\right)\right\} \mathrm{i} \geq 1
\end{aligned}
$$

Therefore, the best mean frequency estimate in the sense of a minimal MSE is the slope of the regression fit through the unwrapped phase. The quality of this estimator is remarkably good but the probability density distribution of the difference in phase $\arg \left\{w_{y}\left(k_{0}, i\right) \cdot w_{y y}^{*}\left(k_{0}, i-1\right)\right\}(2.26)$ leads, as with the IMF estimator, to a biased estimate for high temporal mean frequencies and for wide band signals. The negative influence of the probability density distribution on the quality of this CLR estimator is less than for the IMF estimator due to the regression fit.

\subsubsection{Cross-correlation mean frequency estimator (CC)}

The RF cross-correlation temporal mean frequency estimator (CC) as proposed by (Bonnefous and Pesque 1986) is a non parametric estimator based on the relation between successively sampled RF-signals. To explain the principle of this estimator a dimensionless velocity $h_{i}$ has to be defined as the integerpart of the mean velocity normalized for the ratio between the spatial sampling interval $\Delta y=c /\left(2 . \cos \alpha . f_{s-f f}\right)$ and the temporal sampling interval $\Delta t=l / P R F$ in the RF-matrix:

$$
h_{i}=\operatorname{int}\left(\frac{\bar{v}}{\Delta y / \Delta t}\right)=\operatorname{int}\left(\frac{\frac{c}{2 \cos \alpha} \frac{\bar{f}_{t}}{\bar{f}_{c}}}{\frac{c}{2 \cos \alpha} \frac{P R F}{f_{s-r f}}}\right)=\operatorname{int}\left(\frac{\bar{f}_{i} / P R F}{\bar{f}_{c} / f_{s-r f}}\right)
$$

where $c$ is the speed of sound, $\alpha$ the angle between the ultrasound beam and the velocity

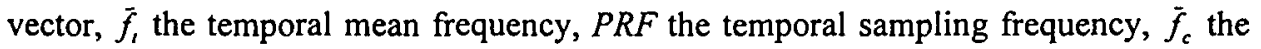
spatial mean frequency and $f_{s-r f}$ the spatial sampling frequency. Employing $h_{i}$ the relation between successive sampled $\mathrm{RF}$-signals $w_{r}(k, i)$ can be defined as:

$$
w_{r f}\left(k_{0}+k, i_{0}+i\right)=w_{f f}\left(k_{0}+k-i \cdot h_{i}, i_{0}+i\right)+n
$$

where $n$ denotes the noise contribution. Based on the assumption of similarity between successive sampled RF-signals the dimensionless velocity $h_{i}$ can be estimated from the peak in cross-correlation

$$
\begin{aligned}
& \hat{R}(j, i)=\frac{1}{M} \sum_{k=-M / 2}^{M / 2-1} w_{r f}\left(k_{0}+k, i_{0}+i\right) \cdot w_{r f}\left(k_{0}+k-j, i_{0}+i+1\right) \\
& -\operatorname{int}\left(f_{s-r} / 2 f_{e}\right) \leq j \leq \operatorname{int}\left(f_{s-r f} / 2 f_{e}\right)
\end{aligned}
$$


between successive sampled RF-signals as:

$$
\hat{\mathrm{h}}_{\mathrm{i}}=\max \{\hat{\mathrm{R}}(\mathrm{j}, \mathrm{i})\}
$$

where $\hat{h}_{i}$ is the estimated dimensionless velocity (spatial shift per temporal sample interval) and $R(j, i)$ the cross-correlation function over the correlation lags $j$ on temporal position $i$, max denotes the maximum operator (peak). The temporal averaging of $\hat{h}_{i}$ in combination with (2.27) leads to the following mean velocity estimator:

$$
\hat{\bar{v}}=\frac{c}{2 \cdot \cos \alpha} \cdot \frac{P R F}{f_{s-r}} \cdot \frac{1}{N} \sum_{i=0}^{N-1} \hat{h}_{i}
$$

The smallest difference between two different mean velocities, being the resolution, of this estimator is limited by the length $N$ of the temporal estimation window and by the ratio between the emission frequency $f_{e}$ and $f_{s-r f}$. A disadvantage of this CC estimator is the huge number of RF-samples necessary to obtain a velocity estimate with a high resolution.

\subsubsection{Cross-correlation model mean frequency estimator (CCM)}

The $\mathrm{RF}$ cross-correlation model mean frequency estimator (CCM) as proposed by (De Jong et al. 1990) is a parametric estimator based on a cross-correlation model of successive sampled RF-signals. To explain the principle of this estimator a dimensionless velocity $\varphi$ has to be defined as the mean velocity normalized for the ratio between the spatial sampling interval $\Delta y=c / 2 \cdot \cos \alpha \cdot f_{s-r f}$ and the temporal sampling interval $\triangle t=l / P R F$ in the RF-matrix:

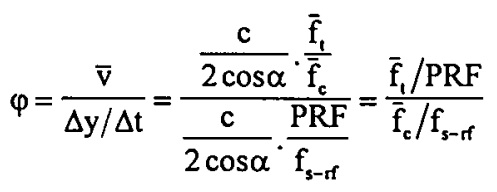

where $c$ is the speed of sound, $\alpha$ the angle between the ultrasound beam and the velocity

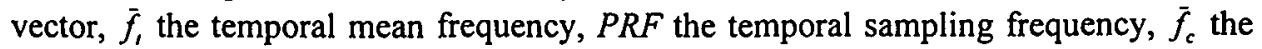
spatial mean frequency and $f_{s-r}$ the spatial sampling frequency. Employing $\varphi$ the relation between successive sampled RF-signals $w_{r}(k, i)$ can be defined as:

$$
w_{r f}\left(k_{0}+k, i_{0}+i\right)=w_{r f}\left(k_{0}+k-i \cdot \varphi, i_{0}+i\right)+n
$$

where $n$ denotes the noise contribution. Based on relation (2.33) and on the assumption of a Gaussian power spectral distribution of the sampled RF-signals, and the same spectral power density distribution of both the RF-signal and the noise, a Gaussian model for the cross-power spectral density distribution $W_{r}(f, i)$ can be defined as: 


$$
\left(G(f, i)=\frac{\left(N_{0}+S_{0} \exp \{-j \cdot 2 \pi f . \varphi . i\}\right)}{\sigma \cdot \sqrt{2 \pi}} \cdot \exp \left\{\frac{-\left(f-\bar{f}_{c} f_{s-r}\right)^{2}}{2 \sigma^{2}}\right\}\right)={ }^{M} W_{r f}(f, i)
$$

where $G$ is the cross-power spectral density distribution of the model, $S_{0}$ the signal power, $N_{0}$ the noise power, $f$ the normalized spatial frequency, $\sigma$ half the normalized spatial bandwidth and $={ }^{M t}$ denotes "is a model for". The cross-correlation function of this model is defined by means of the Wiener-Khinchin relation as:

$$
R(k, i)=\int_{-\infty}^{\infty} G(f, i) \cdot \exp (j \cdot 2 \pi f \cdot k) \cdot d f
$$

hence

$$
\begin{aligned}
& R(k, 0)=\left(S_{0}+N_{0}\right) \cdot \exp \left\{\frac{j \cdot 2 \pi \cdot \bar{f}_{c}}{\bar{f}_{s-r f}} \cdot k\right\} \cdot \exp \left\{\frac{-\sigma^{2} \cdot k^{2}}{2}\right\} \\
& R(k, i)=S_{0} \cdot \exp \left\{\frac{j \cdot 2 \pi \cdot \bar{f}_{c}}{\bar{f}_{s-r f}}(k-\varphi \cdot i)\right\} \cdot \exp \left\{\frac{-\sigma^{2}(k-\varphi \cdot i)^{2}}{2}\right\} \quad i \neq 0
\end{aligned}
$$

Assuming a narrow spatial bandwidth in the model of the cross-power spectral density and taking the real part, the cross-correlation function (2.36) can be simplified to:

$$
R(k, i)= \begin{cases}\left(S_{0}+N_{0}\right) \cdot \cos \left(\frac{2 \pi \cdot \bar{f}_{c}}{f_{s-r f}} \cdot k\right) & i=0 \\ S_{0} \cdot \cos \left(\frac{2 \pi \cdot \bar{f}_{c}}{f_{s-r f}}(k-\varphi \cdot i)\right) & i \neq 0\end{cases}
$$

In order to solve for the unknown model parameters $S_{0}, N_{0}, \bar{f}_{c}$ and $\bar{f}_{1}$ in (2.37) the following cross-correlation lags are considered:

a) $\mathrm{R}(0,0)=\mathrm{S}_{0}+\mathrm{N}_{0}$

b) $R(1,0)=\left(S_{0}+N_{0}\right) \cdot \cos \left(\frac{2 \pi \cdot \bar{f}_{c}}{f_{s \cdot f f}}\right)$

c) $R(0,1)=S_{0} \cdot \cos \left(\frac{2 \pi \cdot \bar{f}_{c}}{f_{s-r}} \cdot \frac{\bar{f}_{1} / P R F}{\bar{f}_{c} / f_{s-r}}\right)$ 
d) $R(1,1)=S_{0} \cdot \cos \left(\frac{2 \pi \cdot \bar{f}_{c}}{f_{s-r f}}\left(1-\frac{\bar{f}_{1} / P R F}{\bar{f}_{c} / f_{s-r f}}\right)\right)$

e) $R(-1,1)=S_{0} \cdot \cos \left(\frac{2 \pi \cdot \bar{f}_{c}}{f_{s-r f}}\left(-1-\frac{\bar{f}_{1} / P R F}{\bar{f}_{c} / f_{s-r f}}\right)\right)$

From those five equations estimators for the model signal parameters are derived as:

$$
\begin{aligned}
& \frac{\hat{\bar{f}}_{c}}{f_{s-r}}=\frac{1}{2 \pi} \cdot \operatorname{acos}(\hat{R}(1,0) / \hat{R}(0,0)) \\
& \frac{\hat{\bar{f}}_{t}}{P R F}=\frac{1}{2 \pi} \cdot \operatorname{atan} 2\left\{\frac{0.5(\hat{R}(1,1)-\hat{R}(-1,1))}{\hat{R}(0,1) \sin \{\operatorname{acos}(\hat{R}(1,0) / \hat{R}(0,0))\}}\right\} \\
& \frac{S_{0}}{N_{0}}=\frac{\hat{R}(0,0) \cdot \cos (2 \pi \cdot \hat{\bar{f}} / P R F)-\hat{R}(0,1)}{\hat{R}(1)} \\
& \hat{\hat{\varphi}}=\frac{\hat{\bar{f}} / P R F}{\hat{\hat{f}}_{c} / f_{s-r f}}=\frac{\operatorname{atan} 2\left\{\frac{0.5(\hat{R}(1,1)-\hat{R}(-1,1))}{\hat{R}(0,1) \sin \{\operatorname{acos}(\hat{R}(1,0) / \hat{R}(0,0))\}}\right\}}{\operatorname{acos}(\hat{R}(1,0) / \hat{R}(0,0))}
\end{aligned}
$$

with $\hat{R}(j, \tau)$ estimated in a given estimation window as:

$$
\begin{aligned}
\hat{R}(j, \tau) & =\frac{1}{(M-j) \cdot(N-\tau)} \sum_{k=0}^{M-i-1} \sum_{i=0}^{N-\tau-1} w_{r i}\left(k_{0}+k, i_{0}+i\right) \cdot w_{r}\left(k_{0}+k+j, i_{0}+i+\tau\right) \\
& 0 \leq j \leq 1 \quad 0 \leq \tau \leq 1
\end{aligned}
$$

The comparison in performance between the LF-domain AC estimator and the CCM estimator, as investigated by (Hoeks et al. 1993), showed that the quality of the CCM estimator is almost a factor two better than the quality of the $\mathrm{AC}$ estimator. The main difference between the $\mathrm{CC}$ estimator and the CCM estimator relates to the difference between non parametric and the parametric approach. The mean velocity $\bar{v}$ in $\mathrm{m} / \mathrm{s}$ is computed from the dimensionless velocity $\hat{\varphi}$ as:

$$
\hat{\bar{v}}=\frac{c}{2 \cdot \cos \alpha} \cdot \frac{P R F}{f_{s-r f}} \cdot \hat{\varphi}
$$




\subsection{Estimator quality comparison}

This section deals with the estimator quality comparison as outlined in Section 2.2.2, for three LF-domain mean frequency estimators. They are based on the AC estimator (Section 2.3.4), MC estimator (Section 2.3.5) and the CLR estimator (Section 2.3.6).

\subsubsection{LF-domain signal space}

The LF-signals $z_{l}(k, i)$ (Section 2.3.1) are composed of three major signal components: reflections $r_{l}(k, i)$, scattering $s_{l f}(k, i)$ and noise $n$ (Fig. 2.6). The signal characteristics of each of these components are different. It may be assumed that the noise $n$ has a Gaussian amplitude distribution and is white and, therefore, uniformly distributed over the temporal frequency range ( $-P R F / 2$ to $P R F / 2$ ). To keep LF-signal simulations simple, it is assumed that both $r_{l}\left(k_{0}, i\right)$ and $s_{l} f\left(k_{0,}, i\right)$ prior to sampling have a Gaussian spectral amplitude distribution, which means they are defined by their amplitude $A$, bandwidth $B$ and temporal mean frequency $F_{t}$, resulting in 6 simulation signal parameters. In addition, the noise amplitude $A_{n}$ and length $N$ of the estimation window should be taken into account, resulting in a signal space (SP) subtended by 8 parameters:

$$
\mathrm{SP}=\left\{\mathrm{A}_{\mathrm{r}}, \mathrm{B}_{\mathrm{r}}, \hat{\overline{\mathrm{f}}}_{\mathrm{r}}, \mathrm{A}_{\mathrm{s}}, \mathrm{B}_{\mathrm{s}}, \hat{\overline{\mathrm{f}}}_{\mathrm{s}}, \mathrm{A}_{\mathrm{n}}, \mathrm{N}\right\}
$$

The quadrature noise series is obtained by the generation of random numbers with a zero mean Gaussian amplitude distribution (mean amplitude $A_{n}$ ) in the time domain (van Leeuwen et al. 1986).
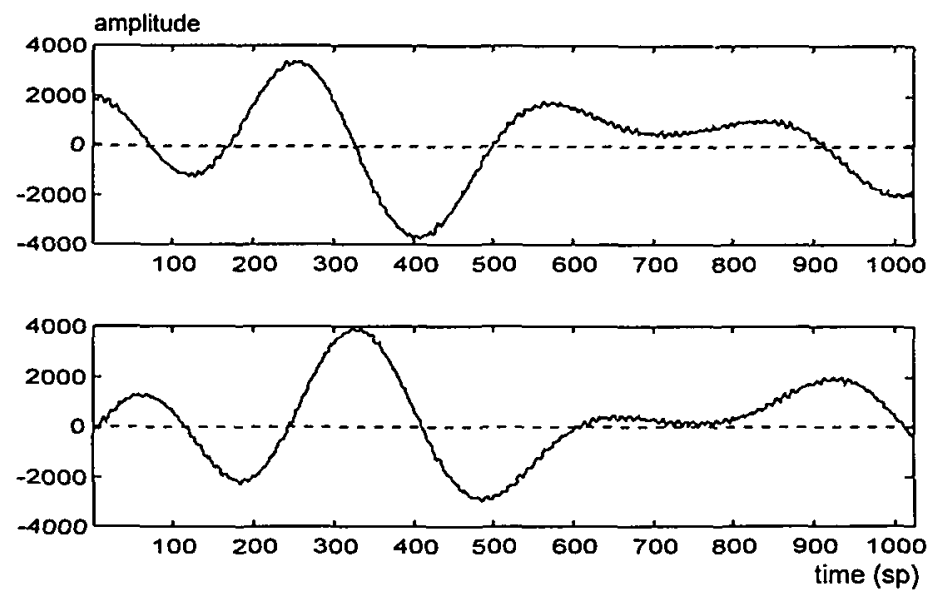

Figure 2.6: The real (top) and imaginary (bottom) part of the simulated LF-signal $z_{y}\left(k_{0}, i\right)$ containing scattering, reflections and noise with a length of 1024 sample points (sp). 
For $r_{l,}\left(k_{0,}, i\right)$ and $s_{l,}\left(k_{0,}, i\right)$, the synthesis starts in the frequency domain with a uniform spectral distribution based on zero mean Gaussian distributed numbers for the real and imaginary components. The spectral distribution is weighed by a Gaussian shaped envelope with the desired bandwidth centred at zero frequency and transferred to the time domain. After appropriate amplitude scaling, the signals are shifted in frequency through multiplication by $\exp \left(-j .2 \pi \cdot i \cdot \bar{f}_{1} / P R F\right)$, where $\bar{f}_{1} / P R F$ is the imposed normalized temporal mean frequency. The signals $r_{l,}, s_{l}$, and $n$ are mixed to obtain $z_{l f}$ (2.6). A large number $(S=200)$ of independent segments in $z_{l f}$ with a length of $N$ sample points are subjected to frequency estimation. Therefore, the signal $z_{l f}$ should have a length of at least $N^{*} S$ sample points for each signal condition $m$ considered. The reflection of the LF-signal in figure 2.6 has an amplitude of $-14 \mathrm{~dB}$, a bandwidth of 0.001 , and a mean frequency of 0.005 , while the scattering has an amplitude of $-44 \mathrm{~dB}$, a bandwidth of 0.05 , and a mean frequency of 0.1 . The noise amplitude is $-54 \mathrm{~dB}$. All frequencies are expressed as a fraction of the pulse repetition frequency (PRF).

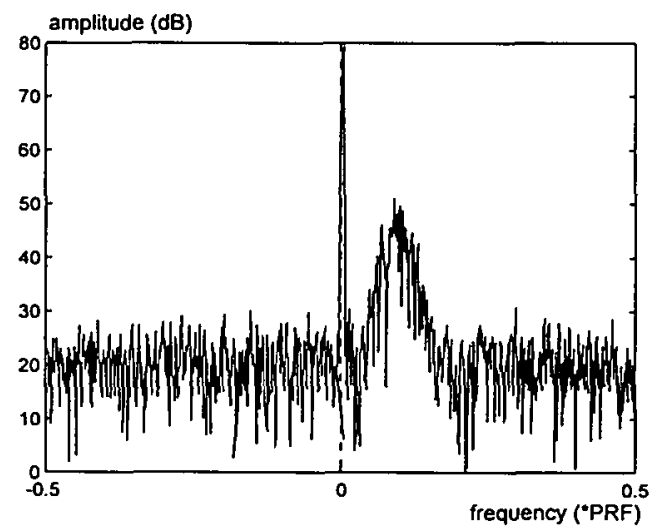

Figure 2.7: Spectral amplitude distribution of the LF-signal $z_{y /}\left(k_{0,}, i\right)$ depicted in figure 2.6.

The signal space considered should reflect realistic situations. Of course, the (timedependent) spectral composition of $\mathrm{zlf}(\mathrm{k} 0, \mathrm{i})$ depends on the location, shape and size of the sample volume. Therefore, Ar will be large if the echo of the wall extends into the sample volume, while Ar will be small for a sample volume situated in the middle of the lumen. Assuming an arbitrary maximum amplitude of $0 \mathrm{~dB}$, suitable values for Ar will be $-4,-14,-24$, and $-34 \mathrm{~dB}$. The bandwidth $\mathrm{Br}$ is always a small fraction of the PRF and may assumed to be constant $(\mathrm{Br}=0.001)$, while the mean frequency of the stationary component $\bar{f}_{r}$ is set at $-0.005,0$, and 0.005 . For a $5 \mathrm{MHz}$ pulsed Doppler system operating at a PRF of $10 \mathrm{kHz}, \bar{f}_{r}=0.005$ corresponds to a velocity of $7.5 \mathrm{~mm} / \mathrm{s}$ along the axis of the ultrasound beam. The scattering slf has a considerably lower amplitude (selected settings $-34,-44,-49$, and $-54 \mathrm{~dB})$, a varying bandwidth $(0,0.05,0.1$, and 0.2$)$ and a mean frequency varying over a wide range $(0.01,0.025,0.05,0.1,0.2$, and 0.4$)$. The higher mean frequencies in combination with a large bandwidth will result in (partial) frequency aliasing. Table 2.1 summarizes the selected parameter settings. 
Table 2.1: Settings considered for each signal parameter, giving a total of 1152 signal conditions. $A_{n}, A_{s}$ and $A_{n}$ are the amplitudes of, the reflection, scattering and noise, respectively. $\bar{f}_{r}$ and $\bar{f}_{s}, B_{r}$ and $B_{s}$ are the mean frequencies and bandwidths of these components. The length of an temporal estimation window in sample points is indicated by $N$.

$\mathrm{SP}=$\begin{tabular}{|c||c|c|c|c|c|c||c||}
\hline $\mathrm{A}_{\mathrm{r}}$ & -4 & -14 & -24 & -34 & & & $\mathrm{~dB}$ \\
\hline $\mathrm{B}_{\mathrm{r}}$ & 0.001 & & & & & & $\mathrm{PRF}$ \\
\hline$\overline{\mathrm{f}}_{\mathrm{r}}$ & -0.005 & 0 & 0.005 & & & & $\mathrm{PRF}$ \\
\hline $\mathrm{A}_{\mathrm{s}}$ & -34 & -44 & -49 & -54 & & & $\mathrm{~dB}$ \\
\hline $\mathrm{B}_{\mathrm{s}}$ & 0 & .05 & .1 & .2 & & & $\mathrm{PRF}$ \\
\hline$\overline{\mathrm{f}}_{\mathrm{s}}$ & 0.01 & 0.025 & 0.05 & 0.1 & 0.2 & 0.4 & $\mathrm{PRF}$ \\
\hline $\mathrm{A}_{\mathrm{n}}$ & -54 & & & & & & $\mathrm{~dB}$ \\
\hline $\mathrm{N}$ & 9 & 17 & 33 & & & & $\mathrm{sp}$ \\
\hline
\end{tabular}

The higher mean frequencies in combination with a large bandwidth will result in (partial) frequency aliasing. The lower mean frequencies are partially suppressed by the discrimination filter. Both effects will degrade the performance of the estimator. For reasons of symmetry, only positive mean frequencies have to be considered. The noise amplitude $A_{n}$ is fixed at $-54 \mathrm{~dB}$, so in the worst case, the signal-to-noise (SNR) ratio will be $0 \mathrm{~dB}$. The total number of signal conditions in $S P$ is the product of the selected values for each parameter. The length of the estimation window $N$ is treated separately because it is under user control. This length may be as short as 9 sample points but is also set at a higher value in subsequent simulation runs to elucidate the effect of longer estimation windows on quality and robustness.

\subsubsection{Results}

A major problem in temporal mean frequency estimation is the presence of vessel wall induced reflections. To illustrate the removal of the vessel wall reflections, depicted in figure 2.6 (temporal length 1024 sample points), from the LF-signal $z_{l,}\left(k_{0}, i\right)$, the signal is split up into estimation windows (segments) with a length of $N=17$ sample points, in addition the reflections and scattering are separated in each estimation window using an adaptive discrimination filter (Chapter 3.3.5). The estimation windows are merged again (to a signal with a length of $1024 \mathrm{sp}$ ) producing the spectral amplitude distribution depicted in figure 2.7. The reflected signal has been completely removed.(Fig. 2.8). Some spectral broadening of the scattered signal due to the filtering process can be noted.In introducing measures for quality and robustness of temporal mean frequency estimators, it is assumed that for a given estimation window with length $N$, all signal parameter values have the same likelihood of occurrence. The results for different estimation window lengths are presented separately in table 2.2. The results shown in figure 2.9 are a reflection of the signal condition sequence in the signal space $S P$. The maximum values for the bias and standard deviation are set at 0.2 and 0.3 , respectively (in fractions of the PRF). If a larger value is observed for a particular signal condition, it 
is set at the maximum value. For the computation of $Q_{e s t}$ and $R_{e s,}$ there is no limitation to bias and standard deviation for particular signal conditions.

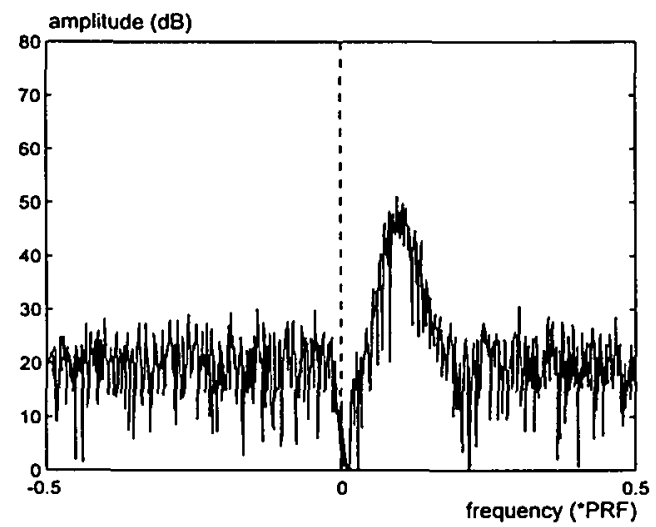

Figure 2.8: Spectral amplitude distribution of the LF-signal depicted in figure 2.6 after the removal of the reflections, using an adaptive discrimination filter.

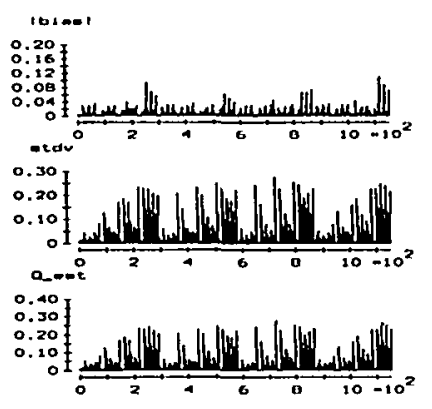

(a)

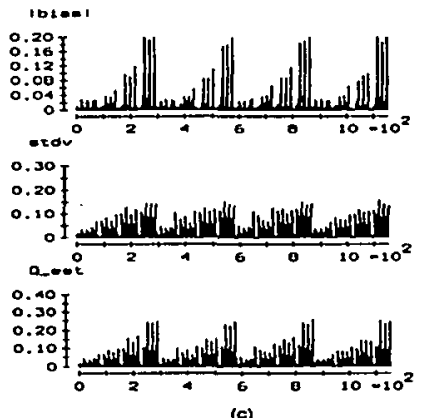

(c)

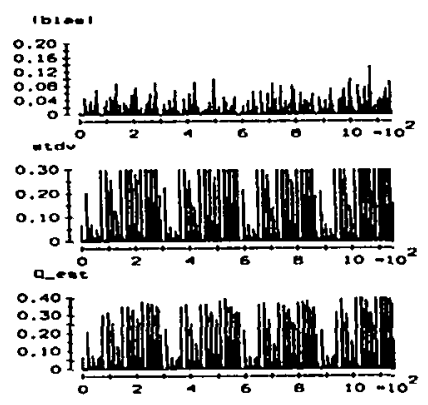

(b)

(top) Bles:

(midalo) Stendard deviletion

(bottom) quelty

Figure 2.9: Observed absolute bias (top), standard deviation (middle) and quality (bottom) for $A C$ (a), MC (b), and CLR (c) as function of signal condition $m$ for an estimation window length of $17 \mathrm{sp}$. 
Table 2.2 lists the results of $Q_{e s t}$ and $R_{e s t}$ for all three frequency estimators for segment lengths of 9,17 and 33 points.

Table 2.2: Results for quality $Q_{\text {est }}$ and robustness $R_{e s t}$ of the estimators AC, MC and CLR for estimation windows of 9,17 and 33 sample points. ( $Q_{e s t}$ and $R_{\text {est }}$ are considered in a negative development smaller values denote better results).

\begin{tabular}{|c|c|c|c|}
\cline { 2 - 4 } \multicolumn{1}{c|}{} & $\mathrm{N}$ & $\mathrm{Q}_{\text {es1 }}$ & $\mathrm{R}_{\text {est }}$ \\
\hline \multirow{3}{*}{$\mathrm{AC}$} & 9 & 0.167 & 0.104 \\
\cline { 2 - 4 } & 17 & 0.078 & 0.066 \\
\cline { 2 - 4 } & 33 & 0.038 & 0.037 \\
\hline \multirow{3}{*}{$\mathrm{MC}$} & 9 & 0.202 & 0.130 \\
\cline { 2 - 4 } & 17 & 0.174 & 0.145 \\
\cline { 2 - 4 } & 33 & 0.158 & 0.156 \\
\hline \multirow{3}{*}{ CLR } & 9 & 0.109 & 0.057 \\
\cline { 2 - 4 } & 17 & 0.068 & 0.050 \\
\cline { 2 - 4 } & 33 & 0.043 & 0.041 \\
\hline
\end{tabular}

\subsubsection{Discussion}

The temporal LF-signal contains scattering induced by flowing blood as well as wideband noise and high amplitude low frequency components induced by reflections originating from moving and stationary structure boundaries. Only the frequency characteristics of the blood induced scattering are of interest. A good frequency estimator should be able to discriminate these characteristics from vessel wall artefacts and noise. In the absence of reflections, it is possible to derive an analytical expression for the variance of the estimates for mean frequency and bandwidth (Kristoffersen 1988). As may be expected, the variance of the estimate based on the auto-correlation function of the Doppler signal with time lag 1 (AC) increases with decreasing SNR ratio, increasing bandwidth and decreasing estimation window length. The expected bias is zero for a symmetric scattering (Doppler) spectrum. Because of the periodic nature of the auto-correlation function, the $\mathrm{AC}$ estimator should be stable for mean frequencies close to the Nyquist frequency, independent of the bandwidth of the scattered signal (van Leeuwen et al. 1986).

Incorporation of a conventional high pass filter to remove reflections makes the result less obvious (Willemetz et al. 1989), especially for a low SNR ratio, because it creates a gap in the spectrum around zero frequency. The approach suggested by Hoeks (Hoeks et al. 1991), based on the slope of the regression line through segments of both the real and imaginary parts of the LF-signal, has the same disadvantage. However, it maximizes the effective length of the estimation window available for mean frequency estimation. The adaptive discrimination filter removes only a single frequency (the one with the largest amplitude around zero frequency) and does not create visible signal discontinuities at the estimation window boundaries (Chapter 3). Nevertheless, in the absence of reflections, the filter will remove another component, most likely originating from the blood. The same consideration applies to the second order auto-regressive 
estimator (MC). Again it is assumed that the level of the reflection is larger than that of the scattered signal. On the other hand, a very large reflected signal interferes with the estimation of the mean frequency of the scattered signal (Ahn and Park 1991), resulting in a biased estimate.

In a simplified model of the ultrasound LF-signal, 7 parameters are identified. The estimation window length is a processing parameter. Depending on the values of these parameters, the observed standard deviation and bias of the estimate will vary. To evaluate the merit of an estimator, the concept of quality $q(m)$ for a specific signal condition $m$ is introduced. The overall quality $Q_{\text {est }}$ in $S P$ is then computed from the mean of $q(m)$ over all signai conditions selected. A large robustness $R_{e s t}$, defined as the root mean square of $q(m)$, is an indication that the estimator is behaving inconsistently.

Signals exhibiting frequency aliasing have a large effect on the measures of bias and standard deviation. As the temporal mean frequency of the scattering approaches the Nyquist frequency, the likelihood that a high mean frequency with opposite sign is temporarily estimated increases, especially in case of wide band signals and/or short estimation windows. This will affect the bias (underestimation) and, particularly, the variance of the estimate for that signal parameter setting. Although all three frequency estimators considered permit partial frequency aliasing, provided that the power of the aliased fraction of the signal is lower than the power of the unaliased fraction, a sharp rise in variance is noted for a high mean frequency and a wide bandwidth. The $q(m)$ for an aliased signal condition may be several orders of magnitude larger than that for an unaliased signal condition.

Another situation leading to an exceptionally high $q(m)$ is a scattered signal with a frequency distribution close to that of the reflected signal (low mean frequency, narrow bandwidth). The discrimination filter may then eliminate both signals, leaving only wide band noise for estimation of temporal mean frequency. The bias in the estimate will still be low (the mean frequency of the noise is zero) but the variance of the estimate will rise (Willemetz et al. 1989). However, in that case the increase in variance is not as dramatic as for frequency aliasing.

A separate problem is the estimation of the temporal mean frequency in the absence of a scattered signal. As in the case of a scattered signal with a frequency distribution resembling that of the reflected signal, the variance of the estimate will increase. A number of options is available to avoid or remove erroneous estimation of the mean frequency. The most simple of these is amplitude thresholding, requiring prior information about the noise level. Another possibility is offered by the estimate for the bandwidth of the signal distribution. A high estimated bandwidth indicates that no dominant spectral peak is observed. A third option to recognise incorrect estimates is the use of two-dimensional image processing, e.g., two-dimensional median filtering. In practice, a combination of the approaches mentioned is used. The signal conditions in this evaluation do not consider the absence of a scattered signal. 
Of course, the results obtained for $Q_{e s t}$ and $R_{\text {est }}$ depend heavily on the selected range of signal parameter values. If only values are selected in the middle of the operating range of the estimator, a favorable $Q_{e s t}$ and $R_{\text {est }}$ will be obtained. The selection of an appropriate range is based on assumptions about the distribution of signal parameter values, and is therefore to some extent arbitrary. However, when comparing the responses of different types of estimators to the same set of signals, $Q_{\text {est }}$ and $R_{\text {est }}$ immediately provide measures of the relative responses of the estimators under consideration.

All estimators considered are yielding a minimal mean squared error in estimating the mean frequency $\bar{f}_{i}$. The results (Table 2.2 ) indicate for all estimators improvement in quality $Q_{e s t}$ for a longer estimation window $N$. The difference in quality and robustness is caused by the difference in the models (AC: one pole within the unit circle, MC: two poles within the unit circle, CLR estimator: one pole on the unit circle). The MC estimator behaves poorly because of the bias in the estimate due to the difference in power between the reflected signal and scattered signal. Ahn and Park (Ahn and Park 1991) solved this problem with a FIR filter to suppress the power of the reflected signal. For this suppression it is better to use a single order estimator in combination with a discrimination filter (Chapter 3). The CLR estimator behaves better than the AC, although the AC is actually better in terms of yielding minimal MSE. The AR model of the AC estimator is based on a pole within the unit circle giving two degrees of freedom, while the AR model of the CLR is based on a pole with a fixed radius $r=l$, giving only one degree of freedom. Therefore, the CLR behaves better than the AC, but the minimal MSE of the CLR is larger than the minimal MSE of the AC estimator. This explains why, for a longer estimation window $(N=33)$, the $\mathrm{AC}$ is better in quality $Q_{\text {est }}$ and robustness $R_{e s t}$ than the CLR.

\subsection{Conclusion}

In this Chapter the principles of mean frequency estimators for ultrasound RF- and LFsignals and of the global performance of three LF-domain estimators are introduced.

The mean frequency estimators show an evolution from LF-domain model based estimators to RF-domain model based estimators. The reason for this shift is that in the LF-domain model based estimators the frequency dependent attenuation of the RFsignals is neglected (Section 1.4.5). The RF-domain estimators do not have this limitation. Both the RF-domain estimators $\mathrm{CC}$ and the CCM are capable of estimating the temporal as well as the spatial mean frequency. The difference between the $\mathrm{CC}$ and $\mathrm{CCM}$ estimator is that between the non parametric against the parametric estimator approach, respectively. This difference explains why the CC estimator needs much more RF sample points than the CCM estimator for an estimate of the mean velocity with the same quality. 
The global performance of the three LF-domain relevant estimators (AC, MC and CLR) takes into account both the bias and variance of the estimates for a range of signal conditions. The quality $Q_{e s t}$ and robustness $R_{e s t}$ immediately indicate whether one estimator performs better than another. However, the absolute values of $Q_{e s t}$ and $R_{e s t}$ are difficult to interpret because they depend greatly on the signal conditions evaluated. For the conditions considered, the mean frequency estimator based on the regression line through the unwrapped phase of the LF-signal (CLR), together with an appropriate discrimination filter, shows the best performance for short estimation windows $(9,17$ $\mathrm{sp})$. For windows longer than $17 \mathrm{sp}$, the first order auto-regressive estimator (AC) is more appropriate. The performance of the second order auto-regressive estimator (MC) is poor in comparison with that of the other two. 



\section{Chapter 3}

\section{Principles of discrimination filters for ultrasound signal components}

3.1 Introduction. 44

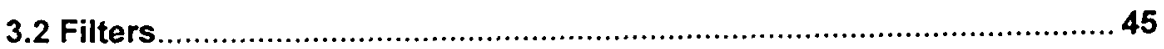

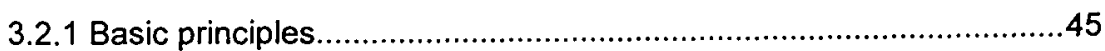

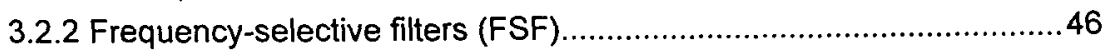

3.2.3 Model based filters (MBF) ......................................................... 48

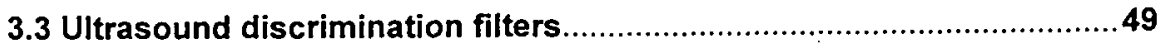

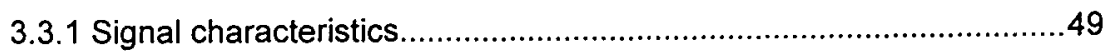

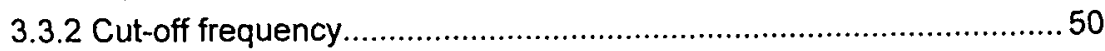

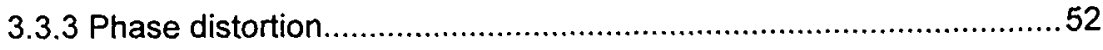

3.3.4 Settling time

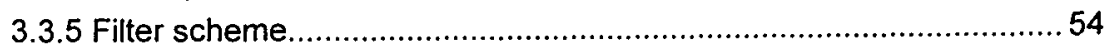

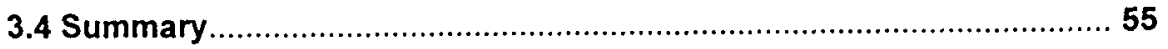




\subsection{Introduction}

The subject of this chapter is to introduce discrimination filters as employed, in RF- and LF-domain ultrasound signal processing, prior to temporal mean frequency estimation with a given single mean frequency estimator (Chapter 2).

$\mathrm{RF}$-signals received consist of the following four major signal components (Fig. 1.6): (1) reflections induced by stationary or slowly moving structure boundaries (clutter), e.g., vessel walls; (2) scattering from moving red blood cells; (3) secondary reflections (reverberations) and (4) noise. The difference in temporal signal characteristics of the reflected and the scattered signal component is utilized for the discrimination between these components. Scattering from red blood cells exhibits a temporal mean frequency depending on the blood flow velocity (Doppler shift), a wide bandwidth and a relatively low amplitude, while reflections have a substantially higher amplitude, a narrow bandwidth and a low or zero temporal mean frequency depending on tissue velocity. For example, the vessel wall velocity of the common carotid artery in the systolic phase is on the order of $7.5 \mathrm{~mm} / \mathrm{s}$. The amplitude of the reflected signal is on the order of 40 $\mathrm{dB}$ higher than the amplitude of the scattered signal. To estimate the temporal mean frequency of the scattered signal it is necessary to suppress the reflected signal (clutter) in the RF- or LF-signal (Section 2.3.1). It is not really a suppression but more a discrimination process because neither the reflected nor the scattered component is actually removed, they are separated. After separation, the temporal mean frequency of both the reflected and scattered signals are estimated with a single mean frequency estimator (Section 2.3). In the case of a dual mean frequency estimator it is not necessary to use a discrimination filter prior to velocity estimation (Section 2.3.5).

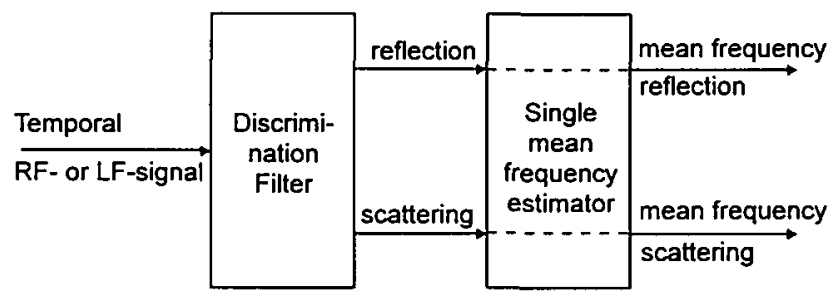

Figure 3.1: Schematic diagram of the ultrasound signal processing used to estimate the mean velocity of both reflectors and scatterers.

An ideal discrimination filter should be able to discriminate without the loss of information, irrespective of the instantaneous difference in amplitude and temporal mean frequency of the signal components subject to discrimination. In the real situation the difference in amplitude, temporal mean frequency and bandwidth will affect the discrimination process because every discrimination filter has a transition width (rolloff) in frequency where discrimination is not selective. For a low ratio in amplitude and a great difference in temporal mean frequency, as compared with the transition width of 
the filter, the discrimination will be selective and without loss of information. However, if the difference in amplitude is great or the difference in temporal mean frequency is small, as compared with the transition width of the filter, the discrimination process is not selective and there will be loss of information. This situation, high ratio between the amplitudes and a comparable temporal mean frequency, is possible within temporal RFor LF-signals originating from the vessel wall lumen boundary. A consequence of the ineffective discrimination between reflection and scattering is a limitation in the temporal mean frequency estimation range (mean blood flow velocity range). To which extent this range, ideal from $-P R F / 2$ to $P R F / 2$, is limited will be investigated for several discrimination filters in combination with the RF-domain CCM estimator in Chapter 4.

This chapter will explain the principles of filters. The different filter types and their notation will be explained. Moreover, the problems related to the design of discrimination filters for ultrasound signal processing are introduced.

\section{$3.2 \quad$ Filters}

\subsubsection{Basic principles}

The purpose of a filter is to separate or remove certain signal components in a signal consisting of different types of signals. The term signal is used to denote the recording in time of a given process, e.g. the ultrasound RF-signal from a given depth, i.e. delay with respect to emission. Every signal can be considered to consist of two or more different signal components, e.g. a given process variable plus noise.

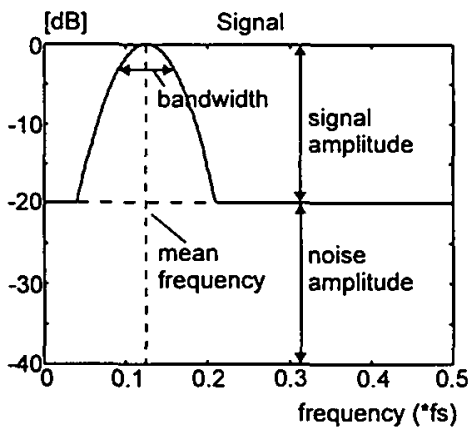

(a)

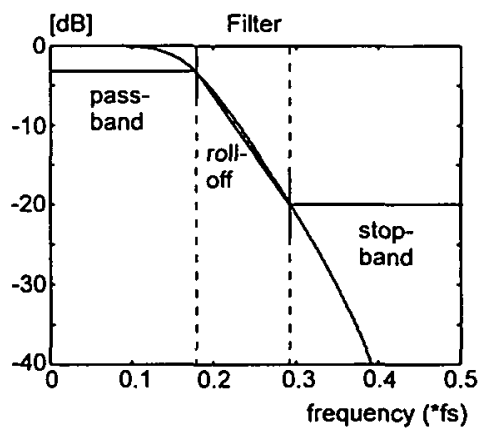

(b)

Figure 3.2: Schematic diagram of the characteristics of a given signal (a) and the characteristics of a given frequency-selective filter (b).

The filter, to separate or remove a signal component from a given signal, is based on general assumptions regarding the signal component (process variable) subject to filtration. The most common filter class in use for this task are the frequency-selective filters (FSF), designed from frequency domain specifications of the various signal 
components, allowing only certain specific frequency bands to pass through. The FSF characteristics as width of pass-band or stop-band region, roll-off and central frequency are based on general assumptions of the mean frequency, bandwidth and amplitude of a given signal component (Fig. 3.2). If the filter characteristics are based on correct assumptions the filtration is effective and there is no loss of information due to filtration. It is not always possible to separate the different signal components effectively and without loss of information because the different signal components may have an overlap in frequency, e.g. reflected and scattered signals in a temporal RF- or LF-signal originating from close to the lumen vessel wall boundary.

An other class of filters, mainly used to remove unwanted signal components from a given signal, are the model based filters (MBF), designed from a model of a given signal component. The model of the MBF is based on prior knowledge of the signal component subject to filtration. The model parameters are estimated within a given filtration window. The MBF is very effective if the model based signal has a high correlation with the signal component to be removed. Singular value decomposition (SVD) filters are filters of the MBF class (Callaerts et al. 1990, Muijtjens et al. 1990). In the SVD approach signals subject to filtration are arranged in a matrix (RF- or LFmatrix) from which an estimate of the common (simular) components is made by means of SVD. A great advantage of SVD is that it is not necessary to assume a model but that the model, in the form of singular values, is estimated directly from the signal subject to filtration. The filtration with MBF is obtained by means of the difference between the model of a given signal component and the signal subject to filtration. A disadvantage of $\mathrm{MBF}$ is that it requires intermediate storage of the signal as vector or matrix in order to estimate the model parameters.

\subsubsection{Frequency-selective filters (FSF)}

The characteristics of an analogue frequency-selective filter (FSF) are obtained by the input-output relationship by means of a differential equation or in the S-domain by means of the Laplace transformation. The characteristics of a digital FSF are obtained by the input-output relationship by means of a difference equation or in the Z-domain by means of the $Z$ transformation (Oppenheim and Schafer 1975).

$$
\begin{array}{ll}
H(s)=\frac{Y(s)}{X(s)} & H(z)=\frac{Y(z)}{X(z)} \\
G(s)=\int_{0}^{o} g(t) \cdot \exp (-s \cdot t) \cdot d t & G(z)=\sum_{i=0}^{\infty} g(i) \cdot z^{-i} \\
s=j .2 \pi . f & z=\exp \left(j \cdot 2 \pi . f / f_{s}\right)
\end{array}
$$

In these equations $s$ denotes the S-domain, $z$ the Z-domain, $y$ the output signal, $x$ the input signal, $g(t)$ a given analogue signal, $g(i)$ a give discrete signal, $f$ frequency, $f_{s}$ the sample frequency, $H(s)$ a filter transfer function in the S-domain and $H(z)$ a filter transfer function in the Z-domain. The digital FSF can be divided into two types: the 
finite impulse response filters (FIR-filters) and the infinite impulse response filters (IIRfilters). IIR filters are recursive and described in general form by the following difference equation:

$$
\begin{aligned}
y(i)= & a_{0} \cdot x(i)+a_{1} \cdot x(i-1)+\ldots+a_{k} \cdot x(i-K)+ \\
& b_{1} \cdot y(i-1)+b_{2} \cdot y(i-2)+\ldots+b_{F} \cdot y(i-F) \quad F \geq K
\end{aligned}
$$

or in the Z-domain by the general transfer function

$$
H(z)=\frac{\sum_{k=0}^{k} a_{k} \cdot z^{-k}}{\sum_{f=0}^{F} b_{f} \cdot z^{-r}} \quad b_{0}=1 \quad F \geq K
$$

where $K$ is the order of the denominator, $F$ the filter order (order of the nominator) and $a_{k}$ and $b_{f}$ are the characteristic filter parameters. FIR filters are given in general form by the following difference equation:

$$
y(i)=a_{0} \cdot x(i)+a_{1} \cdot x(i-1)+\ldots+a_{k} \cdot x(i-K)
$$

or in the Z-domain by the general transfer function

$$
H(z)=\sum_{k=0}^{k} a_{k} \cdot z^{-k}
$$

where $K$ is the filter order and $a_{k}$ are the characteristic filter parameters. The characteristic filter parameters, either from an IIR or FIR filter, are related to the signal component characteristics. The filter can be designed with some mathematics in the Zdomain or with filter design programs such as available from Matlab' .

The cutt-off frequency of the high-pass filter in Fig. 3.3 is chosen in accordance with the roll-off of the filter in use and the amplitude of the signal component to be suppressed. A cutt-off frequency to realize a suppression of $40 \mathrm{~dB}$, with a high-pass filter with a roll-off of $12 \mathrm{~dB} /$ octave, should be at least 3 octaves or a factor 8 (corresponding to 3 times $12 \mathrm{~dB} /$ octave) higher than the mean frequency of the signal component to be suppressed. The FIR filters have both advantages and disadvantages as compared to IIR filters. FIR filters can have exactly linear phase, are always stable and can be realized efficiently in hardware while the filter startup transients are finite in duration. A disadvantage of FIR filters is that they require a much higher filter order than IIR filters to achieve a given roll-off (Fig. 3.3). Moreover, they require intermediate storage of samples up to the order of the filter.

\footnotetext{
${ }^{1}$ Matlab is a registrated trademark of the Math Works Inc.
} 
[db] magnitude

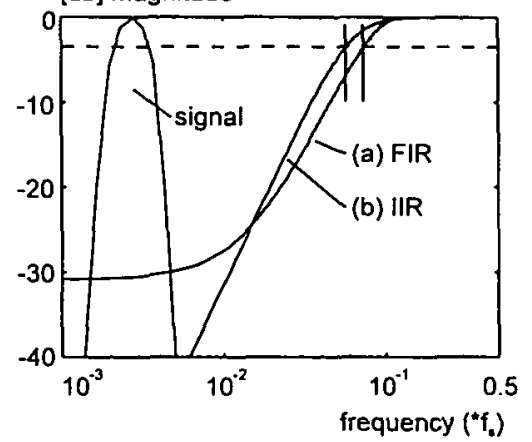

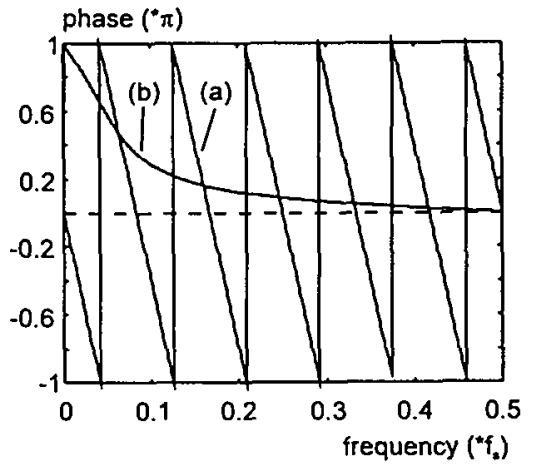

Figure 3.3: The frequency response of a high-pass filter removing a given signal component, designed as a $20^{c}$ order FIR filter (a) or as a $2^{c}$ order IIR filter (b).

The nonlinear phase distortion (Fig. 3.3) is a disadvantage of IIR filters but this is no problem for signals scattered by blood cells where the cell position is random. IIR filters have long lasting transients but they are very efficient to construct.

\subsubsection{Model based filters (MBF)}

Filtration with a model based filter (MBF) is obtained by means of subtraction in the time domain of the model based signal from the signal with the unwanted component in a given filtration window as:

$$
y(i)=x(i)-q(i) \quad i=0 \ldots . N-1
$$

where $x(i)$ is the input signal, $q(i)$ the model based signal, $N$ the length of the filtration window and $y(i)$ the output signal being the result of filtration. In the SVD approach the same procedure is followed but then with matrices.

The characteristics of an MBF are obtained by the expected value of the input-output relationship and the variance on this relationship in the Z-domain (Shanmugan and Breipohl 1988)

$$
\begin{aligned}
& H_{M B F}(z)=E\left\{\frac{Y(z)}{X(z)}\right\} \\
& V_{M B F}(z)=E\left\{\left(\left|\frac{Y(z)}{X(z)}-H_{M B F}(z)\right|\right)^{2}\right\}
\end{aligned}
$$

where $V_{M B F}$ is the variance in the magnitude of the filter and $H_{M B F}$ the expected transfer function. The latter can be modelled with a fit of a digital FSF, either a FIR or an IIR 
filter. This model of the MBF can be used to obtain information about the filter performance. In most cases, it will be problematic or impossible to arrive at an analytical expression for the expected transfer function and the variance on this function. Hence, one has to resort to experimental evaluation of the filter process by means of computer simulations (Hoeks et al. 1991).

\subsection{Ultrasound discrimination filters}

\subsubsection{Signal characteristics}

The real temporal RF-signals $z_{r}\left(k_{0}, i\right)$ (Section 2.3.1), where $k_{\theta}$ denotes a given depth and $i$ denotes time, are in characteristics similar to the complex temporal LF-signals $z_{l}\left(k_{0,}, i\right)$ (Fig. 3.4).

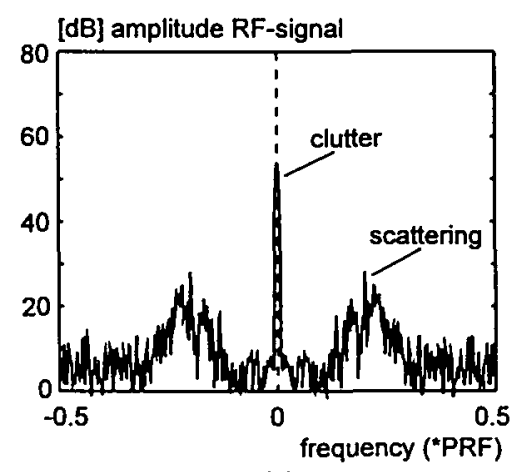

(a)

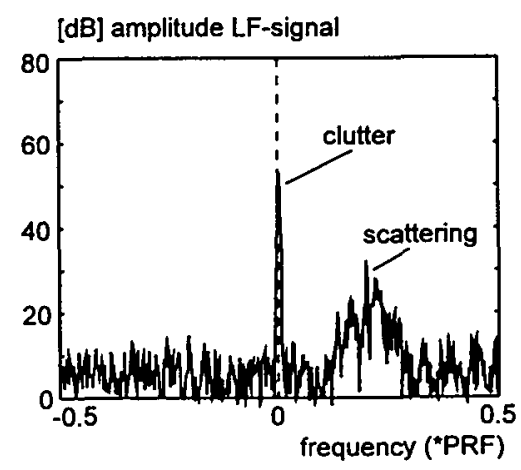

(b)

Figure 3.4: The spectral amplitude density distribution of a signal from a temporal intersect in an RF-matrix $z_{r f}\left(k_{0, i}\right)$ (a) and of a signal from a temporal intersect in an LF-matrix $z_{l},\left(k_{m}, i\right)$ (b).

Because of the similarity in the frequency domain between the temporal RF- and LFsignals the index $r f$ or $l f$, to denote an RF- or LF-signal (Section 2.3.1), can be omitted. The signal characteristics of the clutter $r\left(k_{0}, i\right)$, either an RF- or LF-signal, are: 1) relatively high amplitude, 2) narrow bandwidth, 3 ) low temporal mean frequency. The signal characteristics of the scattered signal $s\left(k_{0}, i\right)$ are: 1) low amplitude, 2) bandwidth depending on variance in velocity and transit time broadening, 3) temporal mean frequency depending on the mean blood flow. The amplitude of the clutter component is on the order of $40 \mathrm{~dB}$ higher than the amplitude of the scattered signal. A realistic value for the temporal mean frequency range and bandwidth of the clutter are $40 \mathrm{~Hz}$ and $15 \mathrm{~Hz}$, respectively. Since the vessel wall has a single velocity the bandwidth of the clutter only depends on the spectral broadening, caused by the transit time effect and acceleration in the movement of the wall. The transit time is caused by the transit of the vessel wall through the ultrasound beam. The temporal mean frequency of the scattered signal depends on the velocity of the scattering particles (red blood cells). The 
bandwidth of the scattered signal depends on the velocity distribution, the acceleration of the scattering particles and the transit time of the particles through the sensitivity region. The latter is responsible for the increase in bandwidth, especially notable at the higher temporal mean frequencies (higher mean velocities) (Fig. 3.5b). A realistic bandwidth range for the scattered signal is difficult to define. For particles moving along the ultrasound beam the temporal quality factor, the ratio between the temporal mean frequency $\bar{f}_{t}$ and the temporal bandwidth $B_{t}\left(Q_{t}=\bar{f}_{t} / B_{\text {, with }} Q_{t}\right.$ the temporal quality factor), equals the quality factor $Q$ of the ultrasound system (2.5) (Section 2.3.1).

$$
Q_{1}=Q
$$

So for a system with a higher axial resolution for the same temporal mean frequency the temporal bandwidth will be greater. A typical example is a system with a quality factor of 2, yielding a temporal bandwidth of $600 \mathrm{~Hz}$ for a temporal mean frequency of 1.2 $\mathrm{kHz}$. The amplitude of the scattered signal is on the order of 10 to $30 \mathrm{~dB}$ higher than the amplitude of the noise. It should be noted that the amplitudes are specified in the time domain.

The overlap in temporal mean frequencies of the reflected signal and the scattered signal (Fig. 3.5a) makes it impossible to discriminate with a frequency selective filter between the two signal components without a significant loss of information for low temporal mean frequencies (low velocities) of the scattered signal.

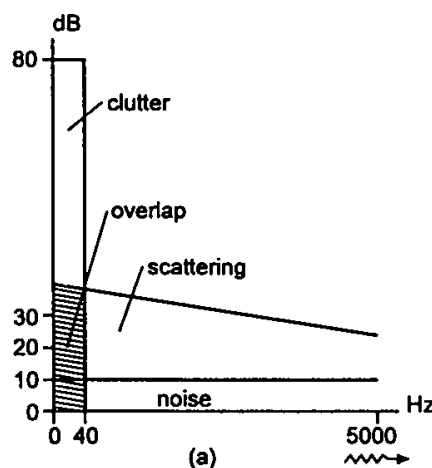

(a)

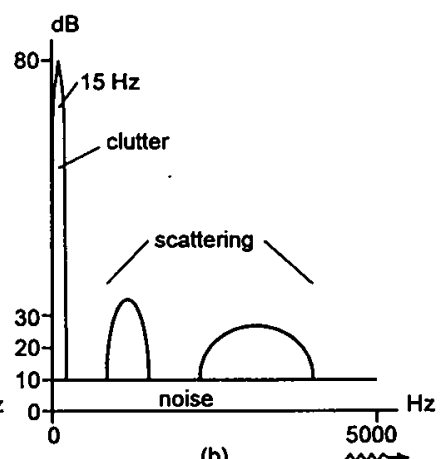

(b)

Figure 3.5: The temporal mean frequency and amplitude range (a) and the bandwidth range (b) of the reflected signal (clutter) and of the scattered signal.

In Fig. $3.5 \mathrm{~b}$ there is only a single scattered signal component but for purpose of demonstrating the effect of bandwidth as function of the temporal mean frequency two components at different mean frequencies are shown. 


\subsubsection{Cut-off frequency}

In general the discrimination between the clutter and the scattered signal is obtained by means of a low- and high-pass filter. The LPF, separating the clutter $r\left(k_{0}, i\right)$ from the signal $z\left(k_{0}, i\right)$, is minimally a second order IIR filter with a cut-off frequency based on general assumptions of the vessel wall motion. The relation between the cut-off frequency, the emission frequency $f_{e}$ and the maximum velocity $v_{\max }$ of the clutter is defined by the Doppler relation as:

$$
f_{\text {cut }}=\frac{f_{e}}{P R F} \cdot \frac{v_{\max }}{c} \quad \alpha=60^{\circ}
$$

where $f_{c u t}$ is the normalized cut-off frequency with respect to the $P R F$ and $\alpha$ the angle between the blood vessel and the ultrasound beam. The characteristics of the HPF, separating the scattered signal $s\left(k_{0}, i\right)$ from the signal $z\left(k_{0}, i\right)$, depend on the maximum velocity of the clutter and on the amplitude ratio of the clutter and the scattered signal. To suppress the clutter with a frequency range from 0 to $40 \mathrm{~Hz}$ and an amplitude of at least $40 \mathrm{~dB}$, with a second order IIR HPF (roll-off $12 \mathrm{~dB} /$ octave), the cut-off frequency should be at least $320 \mathrm{~Hz}$ (Fig. 3.3). The relation between the cut-off frequency $f_{\text {cut }}$, the emission frequency $f_{e}$ and the amplitude ratio is given by the Doppler relation as:

$$
f_{\text {cut }}=\frac{f_{c}}{P R F} \cdot \frac{v_{\text {max }}}{c} 2^{\text {no }} \quad \text { no }=\operatorname{int}\left(r_{2} / r_{\text {off }}\right) \quad \alpha=60^{\circ}
$$

where $r_{a}$ is the amplitude ratio and $r_{\text {off }}$ the roll-off per octave. The loss of information in the low temporal mean frequencies is caused not only by the overlap in frequency between the different signal components but also by the width of the transition region between the stop- and the pass-band of the HPF in use (Fig. 3.3).

To reduce the loss of information caused by the roll-off of the HPF in use it is better to employ a higher order filter or to use an adaptive band-stop filter which adapts its rejection range to the temporal mean frequency of the clutter. A great advantage of the adaptation to the temporal mean frequency of the clutter is that the stop-band is related to the bandwidth of the clutter rather than to the maximum temporal mean frequency (maximum velocity) of the clutter. To achieve a suppression of $40 \mathrm{~dB}$ of the clutter with a maximum bandwidth of on the order of $15 \mathrm{~Hz}$ with a second order adaptive band-stop filter (roll-off $12 \mathrm{~dB} /$ octave) the width of the stop-band should be at least $120 \mathrm{~Hz}$. Thus, an adaptive filter to remove the clutter gives a reduction in the possible loss of information ( $120 \mathrm{~Hz}$ stop-band instead of a $320 \mathrm{~Hz}$ stop-band) due to a decrease in the transition width of the filter in use.

With the use of a frequency selective filter every cut-off frequency within the Nyquist frequency range can be realized. Model based filters have a limitation in the minimal cut-off frequency because of the finite length of the filtration window. The relation 
between the length $N$ of this window and the minimal cut-off frequency $f_{c u t}$ can be defined as:

$$
P R F / 2 \geq f_{\text {cut }} \geq P R F / N
$$

Therefore, the length of the filtration window should be chosen in accordance with the PRF and with the cut-off frequency necessary for an effective filtration. Moreover, the temporal charateristics of the signal (non-stationarity) should be taken into account.

\subsubsection{Phase distortion}

In designing an HPF the non-linear phase distortion caused by IIR filters should be taking into consideration. The phase distortion can introduce a bias in the mean frequency estimation of narrow-band signals, e.g. the reflected signal. The phase distortion does not affect the mean frequency estimation in the scattered signal because of the random phase of this signal. In an ideal situation the phase distortion is constant over the total temporal mean frequency range (-PRF/2 to PRF/2). The latter can be realized with the use of a FIR filter but the practical disadvantage of such a filter is its much higher filter order to achieve a given roll-off (Fig. 3.3). The filter parameter to express the phase distortion (group delay) is defined as:

$$
\tau_{8}=\frac{-d \phi(\omega)}{d \omega}
$$

where $\tau_{g}$ is the group delay, $\phi$ the unwrapped phase and $\omega$ the angular frequency.
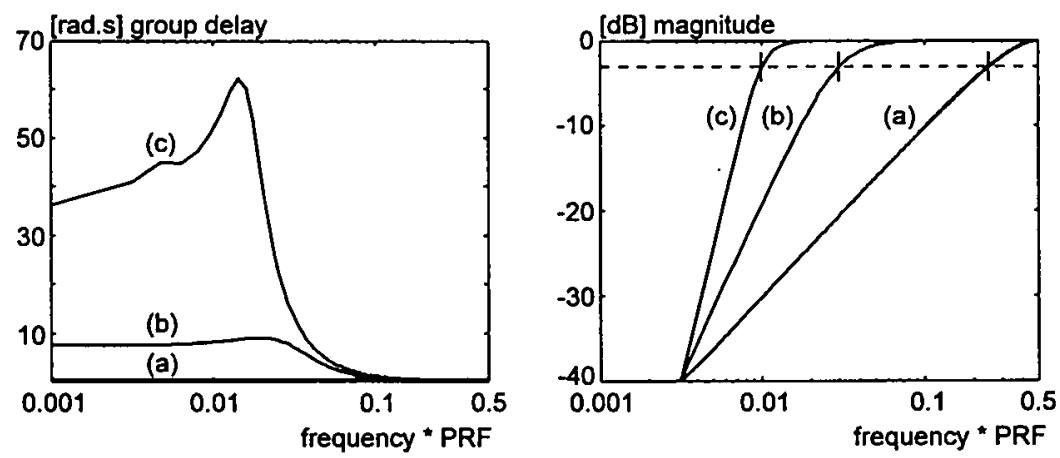

Figure 3.6: The group delay and magnitude of three transfer functions: (a) a $1^{\mathrm{e}}$ order FIR filter, (b) a $2^{\mathrm{e}}$ order IIR filter, and (c) a $4^{\mathrm{e}}$ order IIR filter.

The filters in Fig 3.6 have the same suppression of $40 \mathrm{~dB}$ at a temporal frequency of 0.003 times the PRF. The group delay of the $1^{\mathrm{c}}$ order FIR filter (Fig. 3.6a) is constant (0.5 rad.s) over the whole frequency range but the cut-off frequency to achieve a suppression of $40 \mathrm{~dB}$ is high as compared with the two IIR filters. On the other hand, 
the IIR filters achieve a suppression of $40 \mathrm{~dB}$ with a relatively low cut-off frequency but with a non-linear phase distortion. The phase distortion of the $2^{\mathrm{e}}$ order IIR filter is lower than the phase distortion of the $4^{c}$ order IIR filter.

\subsubsection{Settling time}

The settling time of the discrimination filter is important because during settling time (reaction on a transient in the input signal) the output of the filter can not be used for the estimation of the temporal mean frequency. In M-mode "Doppler" (recording on one place as function of time) the temporal RF- or LF-signal $z\left(k_{0}, i\right)$ is a contiguous function of time without transients in the signal. However, in color flow mappers (Section 1.4.4), recording in a plane as function of time, the temporal RF- or LF-signals $z\left(k_{b}, i\right)$ is contiguous only in a given number of scan lines (8 or 16 lines). In that case each change in direction and/or position of the scan line in the plane of observation will introduce a transition in $z\left(k_{0}, i\right)$ on which the discrimination filter has to settle. This settling time, expressed in sample points, will reduce the effective length $N$ of the estimation window (Section 2.3.1) and so has influence on the estimation quality (Section 2.4.2). FIR filters have a settling time with a finite duration equal to the order of the FIR filter in sample points, while IIR filters have a settling time with a very long duration. The settling time of an IIR filter has to be computed or simulated from the reaction on a step function (Fig. 3.7). It is obvious that in color flow mappers low order FIR filters are used for clutter suppression because of the finite duration of the settling time, accepting the disadvantage of a high cut-off frequency, and the trade-off between the sample points to discard for settling and the limitation in minimal cut-off frequency (3.11).

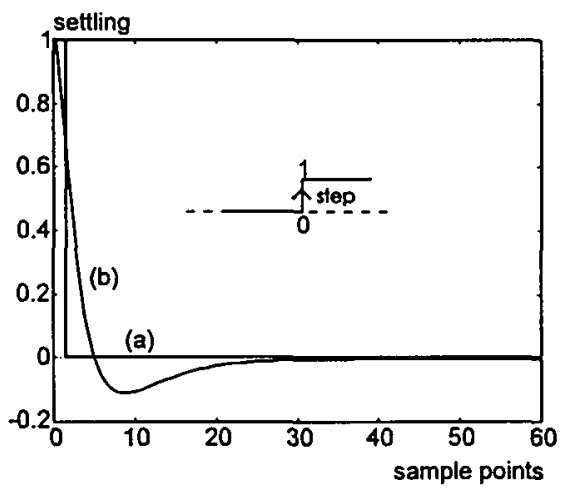

Figure 3.7: The settling time for a first order FIR high-pass filter (a) and for a second order IIR high-pass filter $(b)\left(f_{\text {cut }}=0.04\right)$.

The first order FIR filter in (Fig. 3.7a) has a settling time of one sample point while the IIR filter (Fig. 3.7b) has a settling time of at least 30 sample points. In the case of clutter suppression in color flow mapper applications every FSF will have a disadvantage, 
either for cut-off frequency or settling time. Therefore, an MBF was designed by Hoeks et al. which can suppress clutter with a low cut-off frequency, while the settling time is zero (Section 4.4.4) (Hoeks et al. 1991).

\subsubsection{Filter scheme}

The discrimination of the reflected signals and the scattered signals in RF- or LF-signals can be obtained by means of a static or an adaptive discrimination filter. In the case of a static discrimination filter the filter characteristics are based on general assumptions of the frequency characteristics of the clutter component, i.e., low frequency and a narrow bandwidth. On the contrary, the filter characteristics of an adaptive discrimination filter are based on prior knowledge and actual estimates of the frequency characteristics of the clutter component. The filter scheme of static discrimination filters is simple and consists of (Fig. 3.8a) LP filtration to remove noise and scattered signals from the signal $z\left(k_{0}, i\right)$, and of HP filtration to remove the clutter component from the signal $z\left(k_{0}, i\right)$. The HP filtration is in general obtained with a high order FIR or IIR filter, e.g. a $6^{c}$ order IIR filter. The cut-off frequency of these filters is related to the maximally anticipated velocity (maximum temporal mean frequency) and amplitude of the clutter (3.10). A disadvantage of a high order filter either IIR or FIR is that the higher the order of the filter the higher the bias (systematic error) in the blood flow velocity estimate. The use of a second order IIR filter is a good compromise between the filter performance and the bias in the velocity estimate (Tysoe and Evans 1995).

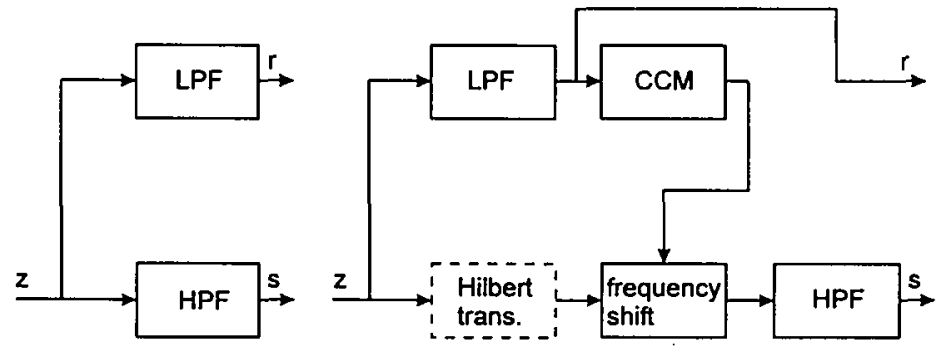

(a)

(b)

Figure 3.8: The filter scheme for a static discrimination filter (a) and for an adaptive discrimination filter (b).

The Hilbert transformation in the adaptive filtration (Fig. 3.8b) is necessary if discrimination is executed in the RF-domain. RF-signals are real signals. However, to perform a frequency shift over the temporal mean frequency of the clutter, the signal should be complex. The filter scheme of adaptive discrimination filters is complex and consists of (Fig. 3.8b) LP filtration, estimation of the temporal mean frequency of the clutter $r\left(k_{0}, i\right)$ and band pass filtration with a central frequency based on the estimated temporal mean frequency of the clutter. The LP filtration, either static or adaptive, is obtained with a $2^{\mathrm{e}}$ order IIR LPF with a cut-off frequency related to the maximum 
velocity (maximum temporal mean frequency) of the clutter (3.9). The estimation of the temporal mean frequency of the clutter (location of the spectral maximum amplitude of the clutter) in the signal $r\left(k_{0}, i\right)$ is for RF-signals obtained with a CCM estimator (Section 2.3.8) and for LF-signals with the CLR estimator (Section 2.3.6). The bandpass filtration is obtained by means of shifting the temporal mean frequency to zero, where the shift is given by the estimated temporal mean frequency of the clutter, and the suppression of the clutter component by an HPF with a cut-off frequency related to the maximum bandwidth of the clutter component.

\subsection{Summary}

In this chapter the methods of discrimination filters for ultrasound RF- and LF-signals as well as the problems related to the design of these filters are introduced.

Discrimination filters are necessary to obtain the temporal mean frequency of the scattered signal with a single mean frequency estimator. Discrimination between the clutter and the scattered signal is achieved by frequency selective filters or by model based filters. In the case of static filtration the filter characteristics of the frequency selective filters and of the model based filters are based on general assumptions of the clutter, i.e. amplitude and temporal mean frequency. In the case of adaptive filtration the filter characteristics are based on the estimated temporal mean frequency of the clutter and on general assumptions of amplitude and bandwidth of the clutter. The main problem in the design of a discrimination filter is the overlap in frequency and the great difference in amplitude $(40 \mathrm{~dB})$ between the clutter and the scattered signal. The overlap in frequency makes it impossible to discriminate with a frequency selective filter without loss of information in the low frequencies. The great difference in amplitude will introduce an even greater loss of information than the overlap in frequency because to suppress clutter of $40 \mathrm{~dB}$ with a filter with a roll-off of 12 $\mathrm{dB} /$ octave the cut-off frequency should be at least a factor of 8 higher than the temporal maximum frequency of the clutter. To reduce the loss of information caused by the rolloff of the filter an adaptive band-pass filter with a central frequency adapted to the temporal mean frequency of the clutter can be used. A great advantage of the latter approach is that the cut-off frequency is related to the bandwidth of the clutter rather than to the maximum mean frequency of the clutter. 



\section{Chapter 4}

\section{The effect of echo suppression on the mean velocity estimation range of the RF cross-correlation model (CCM) estimator}

\subsection{Abstract.} 58

4.2 Introduction 58

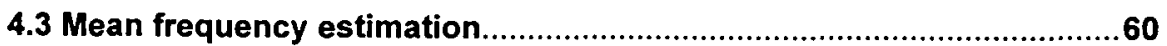

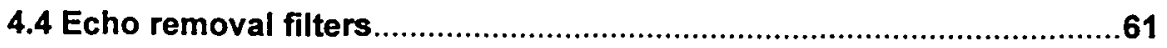

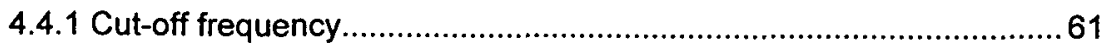

4.4.2 Moving target indicator filter (MTI-f).............................................62

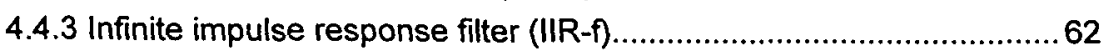

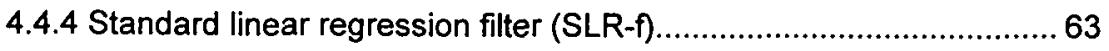

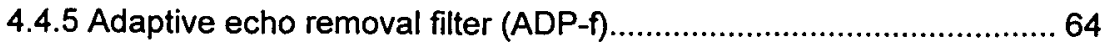

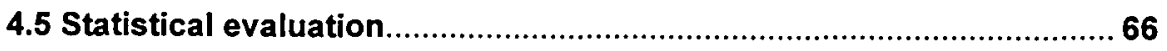

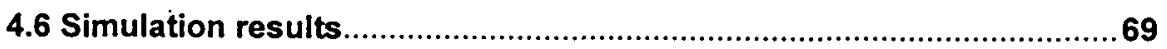

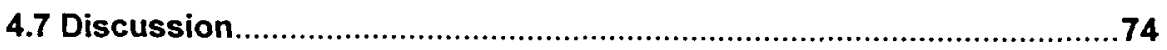

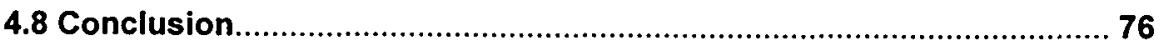

The contents of this chapter is based on the publication: Brands P.J., Hoeks A.P.G., Reneman R.S. The effect of echo suppression on the mean velocity estimation range of the RF cross-correlation model (CCM) estimator. Ultrasound in Med. and Biol., Vol. 21, No. 7, pp. 945-959, 1995. 


\subsection{Abstract}

Ultrasound systems are widely used to visualize in real-time internal structures and blood flow velocity distributions. The latter are estimated from the received ultrasound radio frequent $(\mathrm{RF})$ signals using a mean frequency estimator, capable to estimate the temporal and spatial mean frequency within a given depth and time window (estimation window). Since ultrasound RF-signals are composed of three major signal components (reflections, scattering and noise) it is necessary to suppress the power of the reflections or reverberations (echo removal) to estimate the temporal mean frequency of the signal component induced by scattering originating from red blood cells. However, the echo removal filter in front of the mean frequency estimator will restrict the temporal mean frequency estimation range because the power of the signal component induced by the slowly moving blood is suppressed as well. This chapter describes an echo removal filter, acting in the RF-domain and adaptive to the temporal mean frequency of the signal component induced by stationary or slowly moving reflections and reverberations (clutter). Furthermore, a comparison in performance of this adaptive echo removal filter and several static echo removal filters is presented. For direct comparison of these filter algorithms as to the effect on the temporal mean frequency estimation range, the same signal conditions in combination with the same RF-domain mean frequency estimator were used. It is demonstrated that the adaptive echo removal filter, in combination with the RF-domain cross-correlation model (CCM) mean frequency estimator used, exhibits the best performance.

Key words: clutter suppression; pulsed Doppler; RF cross-correlation; $\mathrm{RF}$-domain signal processing; ultrasound vessel wall filter.

\subsection{Introduction}

The multi-gate pulsed Doppler systems introduced in the late $1970^{\mathrm{s}}$ made it possible to measure in real-time blood flow velocity profiles along a single line of observation (Brandestini et al. 1979, Hoeks et al. 1981). These systems were the predecessors of the color coded Doppler systems showing in real-time the two dimensional blood flow velocity distribution in a plane of observation (Namekawa et al. 1982, Kasai et al. 1985). The fast evolution of mean frequency estimators formed the basis for the fast transition from multi-gate pulsed Doppler systems to color coded Doppler systems (Miller and Rochwarger 1972, Angelsen and Kristoffersen 1983, Bonnefous and Pesque 1986, De Jong et al. 1990, Ferrara and Algazi 1991). Although color coded Doppler systems are very useful tools, the major limitation of these systems is the poor resolution in blood flow velocity estimation, despite the improvement in mean frequency estimators. Restricting the velocity assessment to a single line of observation (multi-gate pulsed Doppler $\mathrm{M}$-line) in assessing the velocity distribution as function of time, the length of the temporal estimation window can be extended. An estimation window of $10 \mathrm{msec}$ ( 80 temporal sample points for a pulse repetition frequency of 8 $\mathrm{kHz}$ ) for an RF-domain mean frequency estimator ensures a low standard deviation of 
the blood flow velocity estimate (good quality) while it does not compromise the temporal behavior of the observed velocities. Despite the very low standard deviation of the velocity estimate, there are still restrictions in the quality of the assessment of blood flow velocity profiles. Most notably, the velocity measurement is angle dependent, and the RF-signals received do not provide information about the perpendicular projection of the blood flow velocity vector on the ultrasound beam. Furthermore, there are limits to the maximally (Hoeks et al. 1981, Jensen 1993) and the minimally detectable velocity. The latter stems from the echo removal filter needed to suppress the power of reflections and reverberations of slowly moving structures, which also suppresses the scattered power of the slowly moving red blood cells.

Normally clutter suppression (echo removal filter) is done with a static high-pass filter, with a fixed cut-off frequency acting in the temporal direction. However, by using such a filter the time dependent aspect of the reflections is ignored. A more selective way is to use a band stop filter which adapts its rejection range to the mean frequency of the clutter. A great advantage of this adaptive method is that the rejection range can be kept small (Section 3.3.2).

This chapter describes an adaptive echo removal filter (ADP-f) acting in the RF-domain and presents a comparison in performance of this ADP-f and the following three static filters: (1) a moving target indicator (MTI-f), (2) a second order IIR filter (IIR-f), and (3) a standard linear regression filter (SLR-f) (Hoeks et al. 1991). While the ADP-f, MTI-f and the IIR-f are frequency selective filters (FSF) the SLR-f is a model based filter (MBF) (Section 3.2). To evaluate the influence of these filter algorithms on the temporal mean frequency estimation range all of them are used in combination with the same RF-domain mean frequency estimator (CCM) (section 2.3.8) (De Jong et al. 1990, Hoeks et al. 1993). An ideal mean frequency estimator should be able to estimate accurately and consistently the temporal mean frequency induced by the moving red blood cells, irrespective of its frequency distribution and the filter algorithm used to suppress the clutter. In practice the frequency distribution will affect the standard deviation (Section 2.4), while the echo removal filter will restrict the minimum temporal mean frequency detectable and induce a bias.

For different signal conditions (like noise level, signal bandwidth, clutter level) and a different filter algorithm the standard deviation of the estimate and the minimally detectable temporal mean frequency will fluctuate. To allow for a direct comparison in performance, as far as the temporal mean frequency estimation range is concerned, the different combinations of filter (MTI-, IIR-, SLR, ADP-f) and estimator (CCM) are evaluated for a common simulated RF-matrix (Fig. 4.1) (RF-signals in time and depth) (Wilson and Gill 1993). The properties of the RF-matrix are described in signal space by a set of temporal and spatial signal parameters constituting the RF-signals. The temporal signal parameters are amplitude, mean frequency and bandwidth of the reflections, scattering and noise. The spatial signal parameters are center frequency and bandwidth of the RF-signal received. 


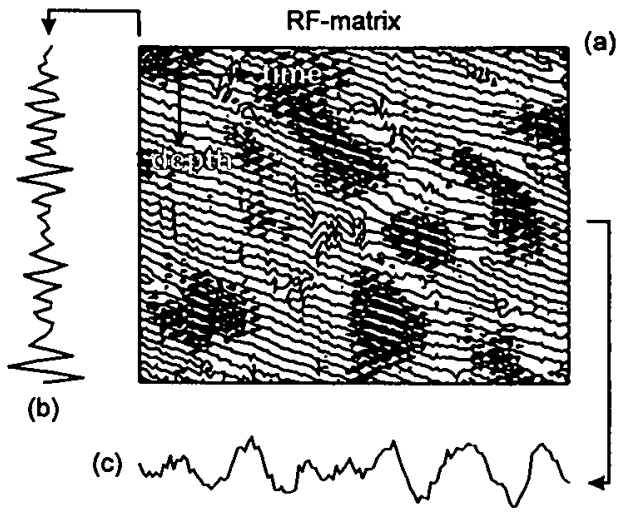

Figure 4.1: (a) A contour map of a simulated RF-matrix in the spatial temporal domain. (b) Signal of a spatial intersect of the RF-matrix. (c) Signal of a temporal intersect of the RFmatrix.

The different filters in combination with the CCM estimator are evaluated repetitively, using 100 estimates based on independent estimation windows in the simulated RFmatrix, resulting in an estimate for the bias (systematic error) and standard deviation of a filter estimator combination. To establish whether a filter modifies the velocity estimate the bias $b_{l}$ and standard deviation $s_{I}$ of the CCM estimator with an echo removal filter are tested statistically (5\% confidence level) against the bias $b_{0}$ and standard deviation $s_{0}$ of the estimates of the CCM estimator without an echo removal filter.

\subsection{Mean frequency estimation}

In the past decades there was a continuous evolution of mean frequency estimators for low frequency (LF) signals (Section 2.3). Recently mean frequency estimators acting in the RF-domain became available (Azaria and Hertz 1984, Bonnefous and Pesque 1986, De Jong et al. 1990) (Section 2.3.7 and 2.3.8).

The RF-domain CCM estimator (Section 2.3.8) is based on a Gaussian model of the spatial cross-power spectral density distribution of received ultrasound RF-signals (De Jong et al. 1990, Hoeks et al. 1993). The estimated RF-domain temporal mean frequency $\hat{\bar{f}}_{1}$, derived from this model (Section 2.3.8), is given by the following equation:

$$
\frac{\hat{\overline{\mathrm{f}}}}{\mathrm{PRF}}=\frac{1}{2 \pi} \cdot \operatorname{atan} 2\left\{\frac{0.5(\hat{\mathrm{R}}(1,1)-\hat{\mathrm{R}}(-1,1))}{\hat{\mathrm{R}}(0,1) \sin \{\operatorname{acos}(\hat{\mathrm{R}}(1,0) / \hat{\mathrm{R}}(0,0))\}}\right\}
$$


where $\hat{R}(k, i)$ is the estimated correlation term from a given estimation window $w_{r}(k, i)$ (Section 2.3.1), $k$ depth and $i$ time. The function atan2( $\div$ ) denotes a double argument arctangent operation. The estimated RF-domain spatial mean frequency $\hat{\bar{f}}_{c}$ derived from this model (Section 2.3.8) is given by the following equation:

$$
\frac{\hat{\bar{f}}_{\mathrm{c}}}{\mathrm{f}_{\mathrm{s}-\mathrm{ff}}}=\frac{1}{2 \pi} \cdot \operatorname{acos}(\hat{\mathrm{R}}(1,0) / \hat{\mathrm{R}}(0,0))
$$

With $f_{s-r f}$ the spatial sampling frequency. The estimated spatial and temporal mean frequency are related to the mean velocity by:

$$
\hat{\bar{v}}=\frac{c}{2 \cdot \cos \alpha} \cdot \frac{P R F}{f_{3-r f}} \cdot \hat{\varphi}
$$

where $\hat{\varphi}$ is the normalized velocity, the ratio of the normalized estimated temporal and spatial mean frequency (Section 2.3.8).

To decide whether estimates are based on noise or on real signals corrupted by noise, a signal-to-noise ratio (SNR) estimator is necessary to provide the relevant information. This SNR-estimator, also derived from the Gaussian model of the spatial cross-power spectral density (Section 2.3.8), is defined by the following equation:

$$
\operatorname{SNR}=\frac{\hat{R}(0,1)}{\hat{R}(0,0) \cdot \cos (2 \pi \cdot \hat{\hat{f}} / P R F)-\hat{R}(0,1)}
$$

where $S \hat{N} R$ is an estimate of the local signal-to-noise ratio.

\subsection{Echo removal filters}

The three components in the raw ultrasound signals (clutter, scattering and noise) can be clearly distinguished in the temporal amplitude spectral distribution of a temporal intersect in an RF-matrix (Fig. 3.4) (Section 3.3.1). To estimate the blood flow velocity with a single mean frequency estimator, the power of the reflections and reverberations, induced by stationary or slowly moving structures, should be suppressed (Section 3.1).

\subsubsection{Cut-off frequency}

The amplitude of the clutter component is on the order of $40 \mathrm{~dB}$ higher than that of the scattered signal induced by blood. For simulation a value of $25 \mathrm{~Hz}$ and $12.5 \mathrm{~Hz}$ is used as maximum temporal mean frequency and bandwidth of the clutter, respectively. In combination with $2.5 \mathrm{kHz}$ as maximum temporal mean frequency of the scattered signal (PRF of $5 \mathrm{kHz}$ ) this will give a normalized temporal mean frequency of 0.005 and a bandwidth of $0.0025\left(\bar{f}_{r}=0.005, B_{r}=0.0025\right.$, Table 4.1). Since the vessel wall has a 
single velocity the bandwidth only depends on the spectral broadening, caused by the transit time effect and acceleration. To realize a suppression of $40 \mathrm{~dB}$ for a given temporal mean frequency of the clutter component the cut-off frequency $f_{\text {cut }}$ should be chosen in accordance with the roll-off of the filter in use (Fig. 3.3) (Section 3.2.2). In order to achieve a suppression of $40 \mathrm{~dB}$ of a clutter component with a temporal mean frequency of 0.005 by means of a second order static high pass filter (maximum suppression at zero frequency, roll-off $12 \mathrm{~dB} /$ octave) the normalized cut-off frequency should be at least 0.04 (Fig. 4.4A). However, if the signal is shifted down in frequency by the center frequency of the signal component induced by the wall, the cut-off frequency can be chosen in accordance with the bandwidth of the clutter component. Therefore, to realize, with the ADP-f a suppression of $40 \mathrm{~dB}$ of the clutter component having a bandwidth of 0.0025 , the normalized cut-off frequency should be at least 0.02 (Fig. 4.4A). This cut-off frequency of $0.02(100 \mathrm{~Hz}$ with a PRF of $5 \mathrm{kHz})$ fits with an estimation window of $10 \mathrm{msec}$.

In 4.4.2 to 4.4.5 a description will be given of the four echo removal filters (MTI-, IIR-, SLR-, ADP-f), which are investigated for their effect on the temporal mean frequency estimation range of the CCM estimator. All filters used were initialized with zero and after allowing adequate time for settling estimates of the filtered output were taken.

\subsubsection{Moving target indicator filter (MTI-f)}

The MTI-f is a static first order FIR high-pass filter with transfer function:

$$
\mathrm{H}(\mathrm{z})=\frac{\mathrm{s}_{\mathrm{rf}}\left(\mathrm{k}_{0}, \mathrm{z}\right)}{\mathrm{w}_{\mathrm{rf}}\left(\mathrm{k}_{0}, \mathrm{z}\right)}=\frac{1}{2} \cdot\left(1-\mathrm{z}^{-1}\right)
$$

where $w_{r f}$ is the input signal, $s_{r f}$ the filtered output signal, $k$ the spatial index (depth) in the RF-matrix and $z$ the $z$-transformation. The cut-off frequency is fixed at 0.25 times the $P R F$ and the roll-off is $6 \mathrm{~dB}$ per octave, giving a suppression of $40 \mathrm{~dB}$ for a temporal mean frequency of 0.004 (Fig. 4.4A). The settling time is only one sample point (Fig. 3.7). The effect of including this echo removal filter prior to a mean velocity estimation based on RF cross-correlation (CC) (Section 2.3.7) was investigated by Jensen (Jensen 1993).

\subsubsection{Infinite impulse response filter (IIR-f)}

The IIR-f is a static second order IIR high-pass filter with the transfer function:

$$
H(z)=\frac{s_{r f}\left(k_{0}, z\right)}{w_{r f}\left(k_{0}, z\right)}=\frac{1}{4} \cdot\left\{\frac{\left(2-b_{0}\right)\left(1-z^{-1}\right)}{1-\left(1-b_{0}\right) z^{-1}}\right\}^{2}
$$

where $b_{0}$ is the characteristic filter coefficient. The cut-off frequency is set at 0.04 times the PRF while the roll-off is $12 \mathrm{~dB}$ per octave, giving a suppression of $40 \mathrm{~dB}$ for a 
temporal mean frequency of 0.005 (Fig. 4.4A). The settling time of this IIR-f is a function of $b_{0}$ (Fig. 4.2).

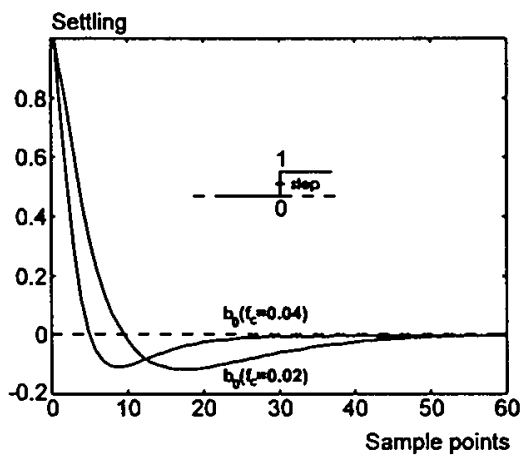

Figure 4.2: The step response of a second order IIR high-pass filter for two different filter coefficients $b_{0}$.

\subsubsection{Standard linear regression filter (SLR-f)}

The $S L R-f$ was specially developed for the use in color coded Doppler systems by Hoeks et al. (1991). While the settling time is zero the $S L R$ echo removal filter can suppress clutter effectively and efficiently. Thus all $N$ sample points within the temporal estimation window can be used for velocity estimation.

The SLR $f$ is based on a first order linear regression model (regression line) of the clutter component within the temporal estimation window $w_{r}\left(k_{0,}, i\right)($ Section 2.3.1) and can be defined as:

$$
\begin{aligned}
& a_{0}(i)+h . a_{1}(i)={ }^{M} w_{r f}\left(k_{0}, i\right) \\
& h=-(N-1) / 2 \ldots . . .(N-1) / 2 \quad N=\text { odd }
\end{aligned}
$$

where $a_{0}$ is the mean of the regression line (intersect), $a_{1}$ the slope of the regression line, $N$ the number of temporal sample points in an estimation window and $={ }^{M}$ "is a model for". It is obvious that this model only holds for short estimation windows as used in color coded Doppler systems. The stationary echo filter based on this linear model is given by:

$$
s_{r f}\left(k_{0}, i\right)=w_{f f}\left(k_{0}, i\right)-\left(\hat{a}_{0}(i)+h . \hat{a}_{1}(i)\right)
$$

where $s_{r}\left(k_{0}, i\right)$ is the filtered temporal RF-signal (the scattered signal). The linear regression coefficients $\left(a_{0}\right.$ and $\left.a_{l}\right)$ are estimated from the RF-matrix within a temporal estimation window as: 


$$
\begin{aligned}
& \hat{a}_{0}\left(k_{0}\right)=\frac{1}{N} \sum_{h} w_{r f}\left(k_{0}, h\right) \\
& \hat{a}_{1}\left(k_{0}\right)=\frac{\sum_{h} h \cdot w_{r r}\left(k_{0}, h\right)}{\sum_{h} h^{2}}
\end{aligned}
$$

where $\hat{a}_{0}\left(k_{0}\right)$ is the estimated $y$-intercept of the regression line and $\hat{a}_{1}\left(k_{0}\right)$ the estimated slope of the regression line. Filtering takes place in successive estimation windows, each with $N$ temporal sample points, in the temporal direction of the RF-matrix for each sample point in depth (spatial direction).

A frequency selective model of the $S L R-f$ (Section 3.2.3) can be obtained with the following expected transfer function:

$$
E\{H(z)\}=E\left\{\frac{s_{r f}\left(k_{0}, z\right)}{w_{r f}\left(k_{0}, z\right)}\right\}=\frac{1}{4} \cdot\left\{\frac{2 N \cdot\left(1-z^{-1}\right)}{N+2-(N-2) \cdot z^{-1}}\right\}^{2}
$$

where $E$ denotes the expectancy operator. The experimental standard deviation of the expected transfer function is shown in Fig. 4.4B. It was not possible to derive an analytic expression for the standard deviation of the expected transfer function. The expected roll-off of this model based high-pass filter is $12 \mathrm{~dB}$ per octave, while the settling time is zero. Filtering is delayed for the time needed to collect sample points to estimate the regression line coefficients ( $N$ times $\Delta t=1 / P R F)$. The expected cut-off frequency for the $S L R-f$ is directly related to the temporal estimation window length and computed by the following equation:

$$
E\left\{\frac{f_{\text {cut }}}{\mathrm{PRF}}\right\}=\frac{1}{2 \pi} \operatorname{acos}\left\{\frac{\mathrm{N}^{2}-12}{(\mathrm{~N}+2)(\mathrm{N}-2)}\right\} \quad \mathrm{N}>3
$$

The normalized cut-off frequency for an estimation window length of 17 sample points is 0.038 giving a suppression of wall artefacts on the order of $40 \mathrm{~dB}$ for a temporal mean frequency of 0.006 times the $P R F$ (Fig. 4.4A).

\subsubsection{Adaptive echo removal filter (ADP-f)}

A more selective way to suppress the clutter is to shift the temporal frequency distribution towards zero frequency where the shift is given by the estimated temporal mean frequency of the reflected signals. Subsequently, the reflections, then centered at zero frequency are suppressed by a high-pass filter with a low cut-off frequency (Fig. $4.4 \mathrm{~A})$. 
To shift the maximum power of the reflections, located at the temporal mean frequency $\bar{f}_{r}$, to zero frequency the real RF-matrix is converted to a complex RF-matrix by a Hilbert transformation performed in the spatial direction (Section 3.3.5). The shift of the temporal mean frequency in the complex RF-matrix is performed by multiplication of every sample point as:

$$
g_{r f}\left(k_{0}, i\right)=z_{r f}\left(k_{0}, i\right) \cdot \exp \left(-j 2 \pi \cdot \hat{\bar{f}}_{r} \cdot i / P R F\right)
$$

where $z_{r}\left(k_{0}, i\right)$ is a sample of the complex RF-matrix, $g_{r}\left(k_{0}, i\right)$ a sample of the zero frequency RF-matrix and $\hat{\vec{f}}_{r}$ (estimated with the CCM estimator) an estimate of the temporal mean frequency of the clutter component.
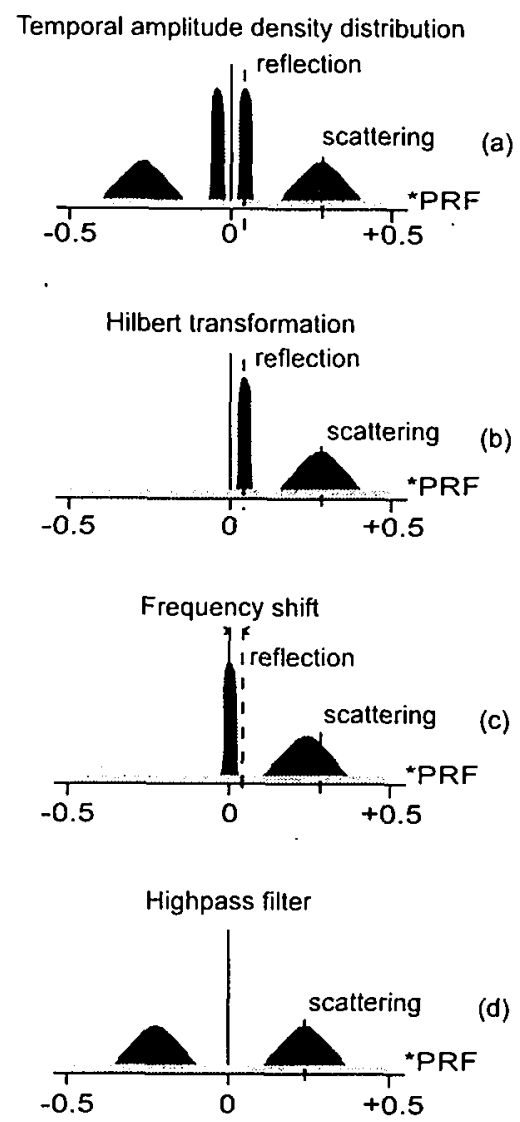

Figure 4.3: Schematic presentation of the main processing steps of an adaptive echo removal filter demonstrated by means of the temporal amplitude density distribution of a temporal intersect in a RF-matrix (a). 
The processing steps in Fig. 4.3 are: (b) Hilbert transformation to convert the real RFmatrix in a complex RF-matrix; (c) shift of the temporal mean frequency of a reflection to zero frequency; (d) high-pass filtration with a low cut-off frequency to suppress the power of the vessel wall reflections at zero frequency. To remove clutter at zero frequency (Fig. 4.3c) the real part of $g_{r}\left(k_{b}, i\right)$ is temporally filtered with a second order infinite impulse response (IIR) high-pass filter with the following transfer function:

$$
H(z)=\frac{s_{r f}\left(k_{0}, z\right)}{\operatorname{RE}\left\{g_{r f}\left(k_{0}, z\right)\right\}}=\frac{1}{4} \cdot\left\{\frac{\left(2-b_{0}\right)\left(1-z^{-1}\right)}{1-\left(1-b_{0}\right) z^{-1}}\right\}^{2}
$$

where $R E\left\{g_{r}\left(k_{0}, i\right)\right\}$ is the real part of $g_{r}\left(k_{0}, i\right), b_{0}$ the characteristic filter coefficient and $s_{r}\left(k_{b}, i\right)$ the filtered temporal RF-signal (the scattered signal). The cut-off frequency is set at 0.02 times the PRF while the roll-off is $12 \mathrm{~dB}$ per octave, giving a suppression of $40 \mathrm{~dB}$ for a temporal mean frequency of 0.0025 (Fig. 4.4A). The settling time of this ADP- $\mathrm{f}$ is a function of $b_{0}$ (Fig. 4.2).

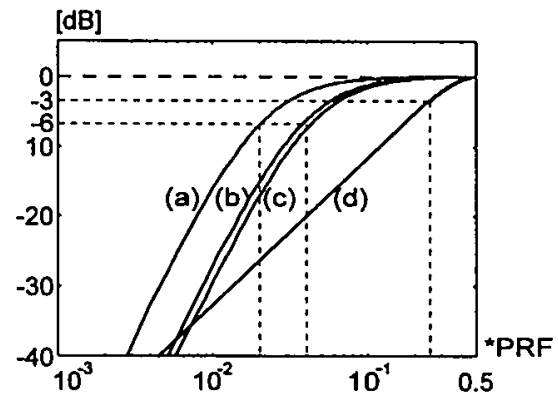

(A)

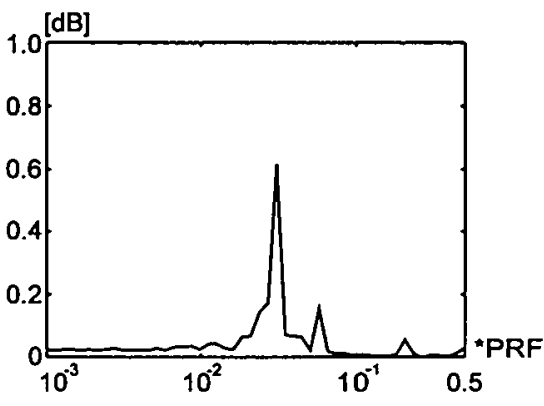

(B)

Figure 4.4: (A) The transfer functions of the different echo removal filters: (a) adaptive echo removal filter with $\hat{\bar{f}}_{r}=0$, (b) standard linear regression filter, (c) second order IIR echo removal filter, and (d) moving target indicator. (B) The standard deviation on the expected transfer function of the standard linear regression filter.

\subsection{Statistical evaluation}

To investigate the difference in temporal mean frequency estimation range, the different combinations of filter and estimator are evaluated for a common simulated RF-matrix with properties described by the parameters in signal space $S P$, subtended by 6 temporal and 2 spatial signal parameters (Table 4.1):

$$
\operatorname{SP}\left(p, \bar{f}_{s}\right)=\left\{A_{r}, B_{r}, \bar{f}_{r}, A_{s}, B_{s}, A_{n}, \bar{f}_{r f}, Q_{r f}\right\}
$$

where $A_{n} B_{r} \bar{f}_{r}$ are the amplitude, bandwidth and temporal mean frequency of the clutter component, respectively, $A_{s}$ and $B_{s}$ the amplitude and velocity bandwidth of the 
blood signal (red blood cells), $A_{n}$ the noise amplitude, $\bar{f}_{r f}$ and $Q_{r f}$ the mean frequency and quality factor $\left(Q_{r F}=\bar{f}_{r} / B\right)$ of the spatial RF-signals, respectively, $p$ a pointer to a given set of signal parameter values and $\bar{f}_{s}$ the imposed temporal mean frequency of the signal component induced by the moving blood.

Table 4.1: Settings considered for each signal parameter giving a total of 4 signal space conditions.

\begin{tabular}{|c|c|c|c|c|c|}
\hline \multicolumn{7}{|c|}{$S P\left(p, \overline{\mathrm{f}}_{\mathrm{s}}\right)$} \\
\hline $\mathrm{p}$ & 0 & 1 & 2 & 3 & unit \\
\hline $\mathrm{A}_{\mathrm{r}}$ & -60 & 10 & 20 & 30 & $\mathrm{~dB}$ \\
\hline $\mathrm{B}_{\mathrm{r}}$ & 0.25 & 0.25 & 0.25 & 0.25 & $\%$ \\
\hline$\overline{\mathrm{f}}_{\mathrm{r}}$ & 0.005 & 0.005 & 0.005 & 0.005 & ${ }^{*} \mathrm{PRF}$ \\
\hline $\mathrm{A}_{\mathrm{S}}$ & 10 & 10 & 10 & 10 & $\mathrm{~dB}$ \\
\hline $\mathrm{B}_{\mathrm{S}}$ & 5 & 5 & 5 & 5 & $\%$ \\
\hline $\mathrm{A}_{\mathrm{n}}$ & 0 & 0 & 0 & 0 & $\mathrm{~dB}$ \\
\hline$\overline{\mathrm{f}}_{\mathrm{rf}}$ & 0.25 & 0.25 & 0.25 & 0.25 & ${ }^{*} \mathrm{f}_{\mathrm{s}}$ \\
\hline $\mathrm{Q}_{\mathrm{rf}}$ & 2 & 2 & 2 & 2 & \\
\hline
\end{tabular}

The simulated RF-matrix was synthesized using the signal generation model described by Hoeks et al. (Hoeks et al. 1993). For all simulations the noise bandwidth $B_{n}$ is equal to the RF bandwidth $\left(Q_{n}=Q_{r f}\right)$. Considering the quality factor $(Q=2 \ldots 4)$ of an ultrasound transducer a spatial sample frequency $f_{s-r f}$ on the order of three or four times the emission frequency is adequate to capture all the available signal information (Section 2.3.1). Figure 4.5 gives a contour map of two simulated RF-matrices with properties $S P(0,0.1)$ and $S P(3,0.1)$ together with a temporal intersect from these RF-matrices. For each set of signal properties the imposed temporal mean frequency $\bar{f}_{\mathrm{s}}$ is logarithmically varied between 0.01 and 0.5 times the $P R F$. This variation is $98 \%$ of the total temporal mean frequency estimation range.

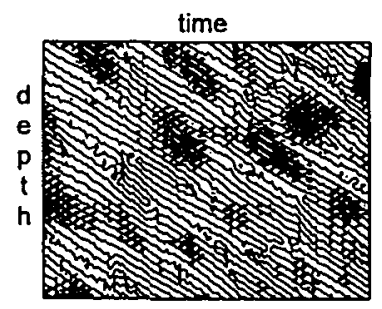

(b)

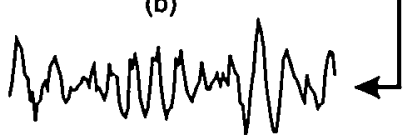

(a)

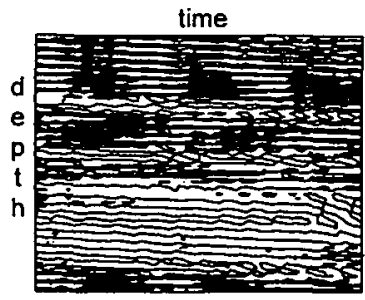

(d)

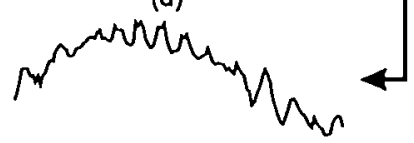

Figure 4.5: Contour maps of simulated RF-matrices with (a) properties $\operatorname{SP}(0,0.1)$ and (c) properties $S p(3,0.1)$, combined with the signals of a temporal intersect respectively (b) and (d). 
Each mean frequency estimate $\hat{\bar{f}}_{s}$, based on successive estimation windows, will have a bias $b_{i}\left(p, \bar{f}_{s}, w_{t}, w_{s}\right)$ and a standard deviation $s_{i}\left(p, \bar{f}_{s}, w_{t}, w_{s}\right)$. Here $w_{t}$ is the temporal length of the estimation window and $w_{s}$ the spatial length of the estimation window (both given in sample points) in a simulated RF-matrix with signal properties $p$ and the true temporal mean frequency $\vec{f}_{s}$. The index $i$ encodes the method of signal processing:

$i=0 \quad$ CCM estimator without echo removal filter
$i=1 \quad$ CCM estimator with echo removal filter

For the CCM estimator without echo removal filter $(i=0)$ signal processing is executed in an RF-matrix with signal property $p=0$ (Table 4.1 ). The CCM estimator with echo removal filter $(i=1)$ employes an RF-matrix with signal properties $p=0,1,2$ and 3 (Table 4.1).

To investigate the restriction in temporal mean frequency estimation range, caused by the use of a certain type of echo removal filter in front of the CCM estimator, the following hypothesis is tested:

$$
\mathrm{H}_{0}: \quad \mathrm{s}_{0}=\mathrm{s}_{1} \quad \text { and } \quad \mathrm{b}_{0}=\mathrm{b}_{1}
$$

where $s_{0}$ and $b_{0}$ are based on the estimates of the CCM estimator without an echo removal filter whereas, $s_{l}$ and $b_{l}$ are based on the estimates of the CCM estimator in combination with an echo removal filter and $H_{0}$ denotes the hypothesis that the echo removal filter has no influence (Fig. 4.6).
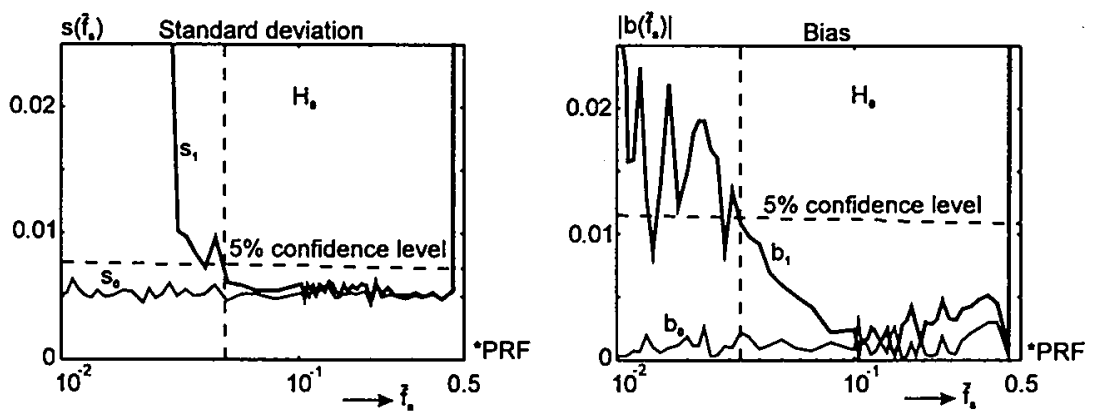

Figure 4.6: The estimates of standard deviation $\left(s_{l}, s_{0}\right)$ and bias $\left(b_{l}, b_{0}\right)$ as function of the temporal mean frequency for the CCM estimator with an echo removal filter $\left(s_{l}, b_{l}\right)$ and the $\mathrm{CCM}$ estimator without an echo removal filter $\left(s_{0}, b_{0}\right)$.

The statistical test is based on the fact that the cross-correlation mean frequency estimate has a normal distribution (Ferrara and Algazi 1991). This implies that the bias 
$b_{1}$ divided by $s_{0}$ is a Student $t$ variable and that the standard deviation $s_{1}$ divided by $s_{0}$ is a chi-square variable. The $H_{0}$ hypothesis of expression 4.16 is accepted if :

$$
\frac{\left|b_{1}\left(p, \bar{f}_{s}, w_{1}, w_{s}\right)\right|}{s_{0}\left(p, \bar{f}_{s}, w_{1}, w_{s}\right)} \leq t_{n-1: a / 2}
$$

and

$$
\frac{s_{1}\left(p, \bar{f}_{s}, w_{1}, w_{s}\right)}{s_{0}\left(p, \bar{f}_{s}, w_{1}, w_{s}\right)} \leq \sqrt{F_{n-1 ; n-1 ; a}}
$$

are true

where $t_{n-1 ; \alpha / 2}$ is the critical region in the Student $t$ distribution, $F_{n-1: n-1 ; \alpha}$ the critical region in the $F$ distribution, $n$ the number of successive estimations $(n=100)$ and $\alpha$ the confidence level $(\alpha=0.05)$. The temporal mean frequency estimation range for each filter is expressed as a percentage of the total temporal mean frequency estimation range and computed as:

$$
\bar{f}_{r a}\left(p, f i l, w_{1}, w_{s}\right)=\frac{\bar{f}_{r a}\left(H_{0}\right)}{0.5} \cdot 100[\%]
$$

where $\bar{f}_{r a}\left(p, f i l, w_{1}, w_{s}\right)$ is the temporal mean frequency estimation range for filter type fil (MTI, IIR, SLR, ADP) and $\bar{f}_{r u}\left(H_{0}\right)$ the temporal mean frequency estimation range where the $\mathrm{H}_{0}$ hypothesis of (4.16) is accepted (Fig. 4.6).

The estimation window for each estimate $\bar{f}_{s}$ is based upon a temporal length $w_{t}$ of 16 or 32 sample points and a spatial length $w_{s}$ of 8 sample points $(Q=2)$. The temporal estimation lengths 16 and 32 sample points is chosen to achieve a normalized cut-off frequency of 0.04 and 0.02 , respectively, for the SLR-f (4.11) (Fig. 4.4A).

\subsection{Simulation results}

Each filter estimator combination (MTI-, IIR, SLR-, ADP-CCM) is statistically tested for the conditions described in the previous section. The simulation results are presented as $s_{i}\left(p, \bar{f}_{s}, w_{t}, w_{s}\right)$ for the standard deviation and as $\left|b_{i}\left(p, \bar{f}_{s}, w_{t}, w_{s}\right)\right|$ for the bias. Note that the latter is an absolute value. Aliasing of Doppler frequencies is the reason for the high standard deviation and the bias for a temporal mean frequency above 0.45 times the $P R F$. The bias and standard deviation are low and slightly increase for an increasing temporal mean frequency. This effect is caused by spectral broadening due to the transit time effect. One should expect a fluctuation for the bias close to zero but this is not the case due to the bandwidth assumption in the derivation of the CCM estimator (Section 2.3.8). The observed bias of the estimate as function of the temporal mean frequency is according to the theoretically bias computed by Hoeks et al. (1993). In the stop band of each echo removal filter the mean frequency of noise is estimated, causing a corresponding standard deviation and bias. The acceptation level of the $H_{0}$ hypothesis 
(4.16) is different for each combination of filter and estimator. For the ADP-CCM combination the temporal mean frequency estimation range is equal to 0.475 times the PRF.
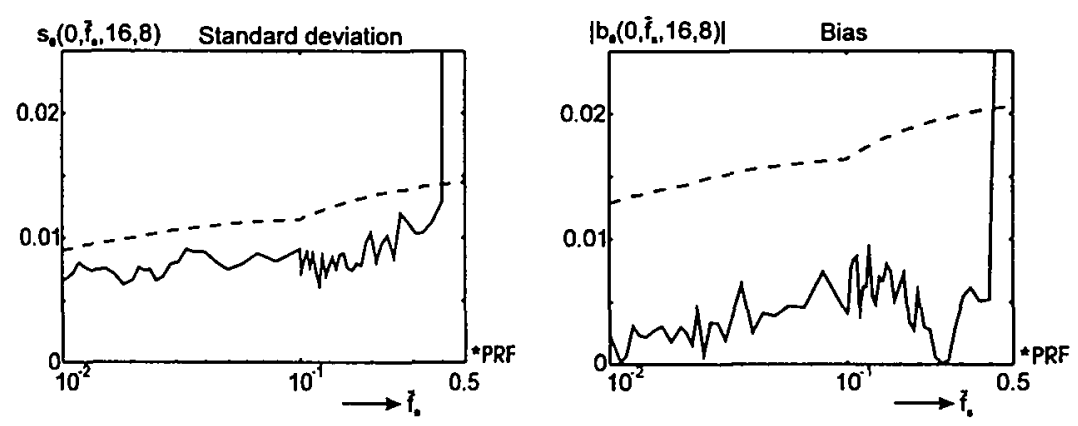

Figure 4.7: The estimates of standard deviation $s_{0}\left(0, \bar{f}_{s}, 16,8\right)$ and bias $\mid b_{0}\left(0, \bar{f}_{s}, 16,8\right)$ as function of the temporal mean frequency for the CCM estimator without an echo removal filter. The dotted line represents the $5 \%$ confidence level.
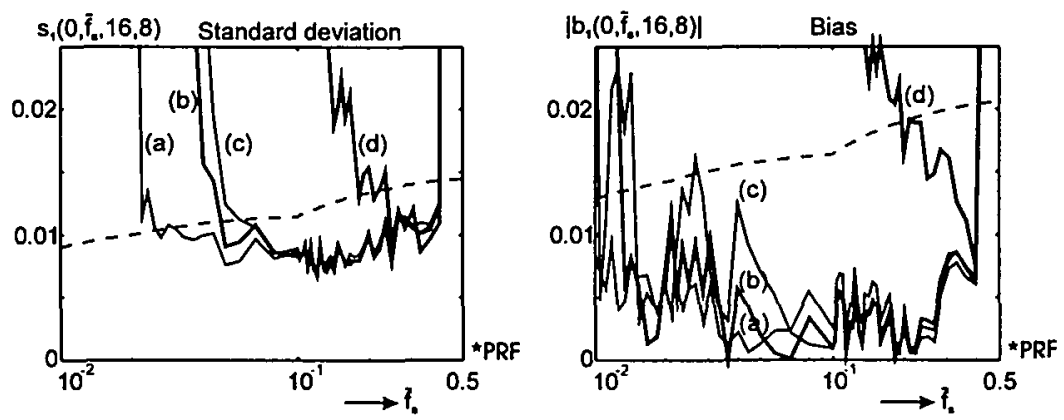

Figure 4.8: The estimates of standard deviation $s_{1}\left(0, \bar{f}_{s}, 16,8\right)$ and bias $b_{1}\left(0, \bar{f}_{s}, 16,8\right) \mid$ as function of the temporal mean frequency for the CCM estimator in combination with (a) an ADP echo removal filter, (b) an SLR echo removal filter, (c) an IIR echo removal filter, (d) an MTI echo removal filter. The dotted line represents the $5 \%$ confidence level.

Expressing the estimation range as a percentage of the total mean frequency estimation range by means of (4.18) gives the following overview for the signal conditions considered

(d) $\mathrm{f}_{\mathrm{ra}}(0, \mathrm{MTI}, 16,8)=50 \%$

(c) $\mathrm{f}_{\mathrm{ra}}(0, \mathrm{IIR}, 16,8)=88 \%$

(b) $\mathrm{f}_{\mathrm{ra}}(0, \mathrm{SLR}, 16,8)=90 \%$

(a) $\mathrm{f}_{\mathrm{ra}}(0, \mathrm{ADP}, 16,8)=95 \%$ 
The adaptive echo removal filter has the best temporal mean frequency estimation range because this filter has the lowest cut-off frequency. For an amplitude of $-60 \mathrm{~dB}\left(A_{r}=-60\right.$ $\left.\mathrm{dB}, S P=\left(0, \bar{f}_{s}\right)\right)$ of the clutter component it is obvious that the filter with the lowest cutoff frequency will have the best temporal mean frequency estimation range.

The temporal mean frequency $\bar{f}_{r}$ of the clutter component is set at 0.005 , indicating that the reflections are moving with a low velocity. In figure 4.9 the temporal estimation window $w_{t}$ is 32 sample points, emphasizing the difference between a static and an adaptive echo removal filter. The adaptive filter will always suppress maximally the clutter component, independent of the temporal mean frequency of this signal component. On the other hand a static filter will only suppress maximally if the signal component has a zero temporal mean frequency (no movement). This difference in filter property, is clearly recognized in figure $4.9 \mathrm{~B}$ where the amplitude of the clutter component is $30 \mathrm{~dB}\left(A_{r}=30 \mathrm{~dB}\right)$. In figure $4.9 \mathrm{~B}$ the temporal mean frequency estimation range for the adaptive filter CCM estimator combination (a) is $96 \%$, which is the same as in figure $4.9 \mathrm{~A}$ for an amplitude of $A_{r}=10 \mathrm{~dB}$. However, the IIR filter CCM estimator combination (c) has a temporal mean frequency estimation range of $0 \%$ in figure $4.9 \mathrm{~B}$ and $90 \%$ in figure $4.9 \mathrm{~A}$.
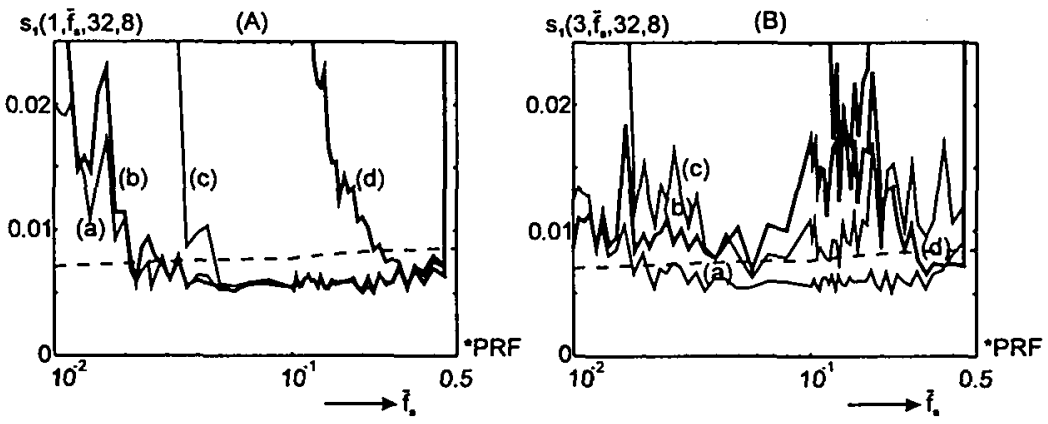

Figure 4.9: The estimates of standard deviation (A) $s_{1}\left(1, \bar{f}_{s}, 32,8\right)$ and $(\mathrm{B}) \mathrm{s}_{1}\left(3, \overline{\mathrm{f}}_{\mathrm{s}}, 32,8\right)$ as function of the temporal mean frequency for the CCM estimator in combination with (a) an ADP echo removal filter, (b) an SLR echo removal, (c) an IIR echo removal filter, (d) an MTI echo removal filter. The dotted line represents the $5 \%$ confidence level.

The cut-off frequency of the MTI-f is fixed at 0.25 times the $P R F$, explaining the short temporal mean frequency estimation range for this combination. The variation of the temporal mean frequency estimation range over the various simulation signal properties $p$ is low, because the suppression of wall artefacts of $40 \mathrm{~dB}$ is only achieved for a temporal mean frequency below 0.004 times the $P R F$. The static character of this MTI-f in combination with the relative high cut-off frequency is reflected in the overall mean $(46.3 \%)$ and the overall root mean square $(5.6 \%)$ of the values in table 4.2 . A low root mean square can be associated with a "good" robustness (independence of signal 
properties), while a high mean value can be associated with a "good" quality in the sense of a "good" temporal mean frequency estimation range (Section 2.2.2).

Table 4.2: The temporal mean frequency estimation range in percentage of the CCM estimator, preceded by a moving target indicator echo removal filter (MTI- $f$ ) for various signal properties p.

\begin{tabular}{|c|c|c|}
\hline \multicolumn{3}{|c|}{$\overline{\mathrm{f}}_{\mathrm{m}}\left(\mathrm{p}, \mathrm{MTI}, \mathrm{w}_{1}, \mathrm{w}_{\mathrm{s}}\right)[\%]$} \\
\hline & \multicolumn{2}{|c|}{$\mathrm{w}_{\mathrm{t}}, \mathrm{w}_{\mathrm{s}}$} \\
\hline $\mathrm{p}$ & 16,8 & 32,8 \\
\hline 0 & 50 & 46 \\
\hline 1 & 46 & 50 \\
\hline 2 & 52 & 50 \\
\hline 3 & 36 & 40 \\
\hline Mean & \multicolumn{3}{|c|}{46.3} \\
\hline RMS & \multicolumn{3}{|c|}{5.6} \\
\hline
\end{tabular}

Table 4.3: The temporal mean frequency estimation range in percentage of the CCM estimator, preceded by an infinite impulse response echo removal filter (IIR-f) for various signal properties $p$.

\begin{tabular}{|c|c|c|}
\hline \multicolumn{3}{|c|}{$\overline{\mathrm{f}}_{\mathrm{ra}}\left(\mathrm{p}, \mathrm{IIR}, \mathrm{w}_{1}, \mathrm{w}_{\mathrm{s}}\right)[\%]$} \\
\hline & \multicolumn{3}{|c|}{$\mathrm{w}_{\mathrm{f}}, \mathrm{w}_{\mathrm{s}}$} \\
\hline $\mathrm{p}$ & 16,8 & 32,8 \\
\hline 0 & 88 & 90 \\
\hline 1 & 88 & 90 \\
\hline 2 & 14 & 31 \\
\hline 3 & 0 & 0 \\
\hline Mean & \multicolumn{3}{|c|}{50.1} \\
\hline RMS & \multicolumn{3}{|c|}{42.7} \\
\hline
\end{tabular}

The cut-off frequency of the IIR-f is set at 0.04 times the $P R F$ and this is reflected in the values of table 4.3. For the simulation signal properties $p=0,1$ the length of the temporal estimation window does not give a significant difference for the temporal mean frequency estimation range, which is on the order of $89 \%$. However, for the signal property $p=2$ the length of the temporal estimation window has an effect on the temporal mean frequency estimation range. This can be explained by the better estimate of the temporal mean frequency for a longer estimation window $w_{t}$. The static character of this IIR-f makes the temporal mean frequency estimation range of this filter estimator combination IIR-CCM dependent on the variation in the simulation signal conditions, which is reflected in the overall mean $(50.1 \%)$ and in the overall root mean square $(42.7 \%)$ of the values in table 4.3 . 
Table 4.4: The temporal mean frequency estimation range in percentage of the CCM estimator preceded by a standard linear regression echo removal filter (SLR-f).

\begin{tabular}{|c|c|c|}
\hline \multicolumn{3}{|c|}{$\bar{f}_{n}\left(p, S L R, w_{1}, w_{s}\right)[\%]$} \\
\hline & \multicolumn{2}{|c|}{$w_{t}, w_{s}$} \\
\hline$p$ & 16,8 & 32,8 \\
\hline 0 & 90 & 95 \\
\hline 1 & 88 & 96 \\
\hline 2 & 86 & 26 \\
\hline 3 & 26 & 0 \\
\hline Mean & \multicolumn{3}{|c|}{63.4} \\
\hline RMS & \multicolumn{3}{|c|}{36.6} \\
\hline
\end{tabular}

Table 4.4 demonstrates that the cut-off frequency of the SLR- $f$ is a function of the temporal estimation window (4.11). The longer the length of the temporal estimation window $w_{t}$, the lower the expected cut-off frequency of the SLR-f will be. For a temporal estimation window of $32 \mathrm{sp}$ the normalised cut-off frequency is 0.019 which is almost equal to the cut-off frequency of the ADP-f. An SLR-f with a cut-off frequency of $0.019\left(w_{t}=32\right)$ gives a suppression of wall artefacts on the order of only $20 \mathrm{~dB}$ for a temporal mean frequency of 0.005 times the $P R F$. The static character of this SLR-f makes it dependent on the variation in signal conditions which is reflected in the overall mean $(63.4 \%)$ and in the overall root mean square $(36.6 \%)$ of the values in table 4.4.

Table 4.5: The temporal mean frequency estimation range in percentage of the CCM estimator preceded by an adaptive echo removal filter (ADP-f) for various signal properties $p$.

\begin{tabular}{|c|c|c|}
\hline \multicolumn{3}{|c|}{$\bar{f}_{\mathrm{ra}}\left(\mathrm{p}, \mathrm{ADP}, \mathrm{w}_{1}, \mathrm{w}_{\mathrm{s}}\right)[\%]$} \\
\hline & \multicolumn{2}{|c|}{$\mathrm{w}_{\mathrm{t}, \mathrm{w}_{\mathrm{s}}}$} \\
\hline $\mathrm{p}$ & 16,8 & 32,8 \\
\hline 0 & 95 & 96 \\
\hline 1 & 94 & 96 \\
\hline 2 & 94 & 94 \\
\hline 3 & 95 & 96 \\
\hline Mean & \multicolumn{3}{|c|}{95.0} \\
\hline RMS & \multicolumn{2}{|c|}{0.9} \\
\hline
\end{tabular}

The temporal mean frequency estimation range in table 4.5 is wide, stable and consistent for the various simulation signal properties $p$. The adaptive nature of this filter makes it almost independent of the variation in signal conditions which is reflected in the overall mean $(95 \%)$ and in the overall root mean square $(0.9 \%)$. 


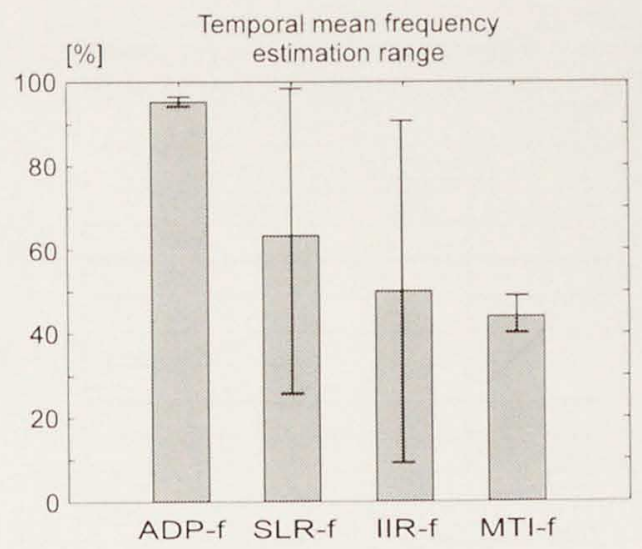

Figure 4.10: The mean and root mean square in percentage of the temporal mean frequency estimation range for the four filter estimator combinations (MTI-, IIR-, SLR-, ADP-CCM) for the simulation signal properties $p$.

Figure 4.10 confirms the excellent estimation range of the ADP-CCM estimator combination.

\subsection{Discussion}

A major restriction for blood flow velocity assessment with ultrasound is the inability to discriminate unambiguously between signals induced by slowly moving structures, like vessel walls, and those induced by slightly faster moving blood near the vessel wall. To overcome this limitation we introduce a cross-correlation model (CCM) estimator preceded by an adaptive echo removal filter to determine near-wall velocity behaviour. The latter is important to be able to estimate reliably wall shear rate (Chapter 5).

To estimate the blood flow velocity from the back scattering of the red blood cells with a single mean frequency estimator, the clutter should be suppressed. Normally this is done with a high-pass filter. However, using such a filter the time dependent aspect of the reflections is ignored. Therefore, we introduce an adaptive echo removal filter which adapts its rejection range to the temporal mean frequency (velocity) of the clutter. A great advantage of the adaptive method over a static echo removal filter is that the rejection range can be kept small. Therefore, the temporal mean frequency estimation range is considerably greater than for a static echo removal filter, the cut-off frequency of which should be set considerably higher than the anticipated maximum temporal mean frequency of the vessel wall to achieve sufficient suppression.

In a simplified model of raw RF-signals, 6 temporal and 2 spatial simulation signal parameters are identified. The temporal and spatial estimation windows $\left(w_{t}, w_{s}\right)$ are processing parameters. The cut-off frequency of a given echo removal filter is set, considering the roll-off and the fact that the amplitude of the clutter component is on the 
order of $40 \mathrm{~dB}$ higher than that of the signal induced by blood. Depending on the simulation signal properties $p$ and the different echo removal filters in front of the CCM estimator, the observed temporal estimation range for the CCM estimator will vary (Tables 4.2, 4.3, 4.4 and 4.5). To evaluate the merit of a combination of CCM estimator and echo removal filter upon the temporal mean frequency estimation range, the mean and root mean square (RMS) over all the simulation signals properties $p$ is computed (Fig. 4.10) (Section 2.2.2). The results obtained for the overall mean and root mean square of the temporal mean frequency estimation range depend heavily on the selected simulation signal parameter values (Table 4.1). However, applying the different filter estimator combinations to the same set of simulation signals, the overall mean and root mean square of the temporal mean frequency estimation range immediately provide a measure of the relative behavior of the filter-estimator combinations.

Except for the MTI-f, all the high pass filters discussed are of a second order and therefore, exhibit a roll-off of $12 \mathrm{~dB}$ per octave. The cut-off frequency should be set a number of octaves higher than the frequency of the signal component to be suppressed (Section 3.3.2). For the MTI-f this results in an exceptionally high cut-off frequency leading to a reduced SNR over a large signal range (Jensen 1993). However, for the ADP-f a very low cut-off frequency, corresponding with the bandwidth rather than the maximum temporal frequency of the clutter, can be selected. It should be noted that both the SLR-f and ADP-f follow a two-step analysis of the rf-matrix. First the signal properties are established while in the second step rejection of the unwanted component is executed.

As the temporal mean frequency of the scattered signal induced by blood approaches the Nyquist frequency (PRF/2), the possibility increases that a high temporal mean frequency with the opposite sign is temporally estimated. Temporal signals exhibiting frequency aliasing have a great effect on the bias and root mean square of the estimate (Fig. 4.6 to 4.9 ). They will also restrict the temporal mean frequency estimation range. In the simulation results the effect of aliasing is not considered, because this restriction is independent and is the same for the different echo removal filters in front of the CCM estimator.

Another situation leading to an exceptionally high bias and standard deviation of the estimate is the estimation of the temporal mean frequency in the absence of a signal component induced by blood flow velocity. The echo removal filter will suppress the clutter component, leaving only wide band noise as input for the CCM estimator. To avoid an exceptionally high bias and standard deviation of the estimate, an estimate of the SNR (4.4) can provide a criterion to decide whether estimates are based on wideband noise or on real band-limited signals. 


\subsection{Conclusion}

The influence of echo suppression with different echo removal filters on the mean frequency estimation range of the CCM RF-domain cross-correlation model estimator was investigated. The removal of stationary echoes from RF-signals, using an echo removal filter prior to the CCM mean frequency estimator, will restrict the measurement of low blood flow velocities. It has been shown that different echo removal filters in combination with the CCM estimator will give a difference in the mean frequency estimation range. For the RF-signal conditions considered the combination of the adaptive echo removal filter CCM estimator shows the best overall mean frequency estimation range of $95 \%$ which range is almost independent of signal properties, reflected in an overall root mean square of $0.9 \%$. The overall mean frequency estimation range for the standard linear regression and the infinite impulse response echo removal filter in combination with the CCM estimator is $63.4 \%$ and $50.1 \%$, respectively. This range is sensitive to the variation in signal properties, reflected in an overall root mean square of $36.6 \%$ and $42.7 \%$, respectively. The combination of moving target indicator echo removal filter and CCM estimator shows an overall mean frequency estimation range of $46.3 \%$ and an overall root mean square of $5.6 \%$. These values show that the traditional static echo removal filters give a large reduction in the mean frequency estimation range. For the adaptive echo removal filter the mean frequency estimation range is considerably greater because the reflections are suppressed by a small band stop filter that apparently adapts its central frequency to the temporal mean frequency of the slowly moving reflections or reverberations. In general the conclusion can be stated that the ADP-f provides a greater temporal mean frequency estimation range than a static filter. The extent of this increase depends on the type of estimator. 


\section{Chapter 5}

\section{A non-invasive method to estimate wall shear rate using ultrasound}

5.1 Abstract. 78

5.2 Introduction

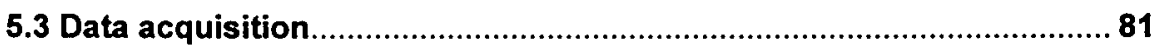

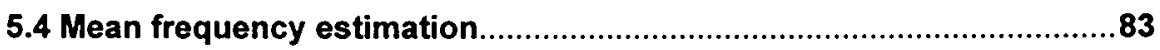

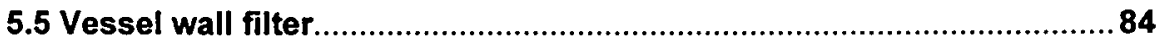

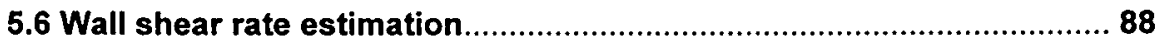

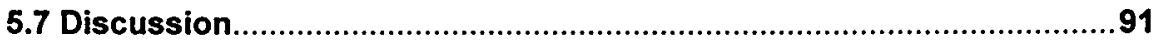

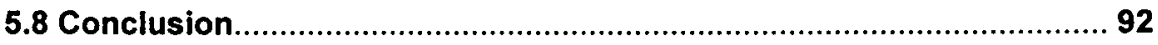

The contents of this chapter is based on the publication: Brands P.J., Hoeks A.P.G., Hofstra L., Reneman R.S. A non-invasive method to estimate wall shear rate using ultrasound. Ultrasound in Med. and Biol. Vol. 21 No. 2, pp. 171-185, 1995 


\subsection{Abstract}

Wall shear stress (blood viscosity times wall shear rate), imposed by the flowing blood, and blood pressure are the main mechanical forces acting on a blood vessel wall. Accurate measurement of wall shear stress is important when investigating the development of vascular disease, since both high and low wall shear stresses have been cited as factors leading to vessel wall anomalies. Furthermore, in vitro studies have shown that endothelial cells, which play a key role in the function of the underlying arterial wall, undergo a variety of structural and functional changes in response to imposed shear stress. However, there is practically no knowledge about the influence of wall shear stress on the arterial wall in vivo because of the difficulty to measure this stress in terms of magnitude and time variation. The method presented in this chapter to measure the time dependent wall shear rate in main arteries is based on the evaluation of velocity profiles determined by means of ultrasound, using off-line signal processing. Pulsed ultrasound is well suited for this application since it is non-invasive. The processing performed in the RF-domain consists of a mean frequency estimator preceded by an adaptive vessel wall filter. In a pilot study ( 6 repeated measurements in the common carotid artery of 5 healthy volunteers) we investigated the reproducibility of the method to estimate peak wall shear rate as compared with the reproducibility of the measurement of peak center-line blood flow velocity. The coefficient of variation for peak blood flow velocity was $9 \%$, and for peak wall shear rate it was $5 \%$.

Key words: pulsed Doppler; RF cross-correlation; shear stress; signal processing; ultrasound; vessel wall filter; wall shear rate.

\subsection{Introduction}

The subject of this chapter is to describe a non-invasive method to estimate wall shear rate using, dedicated RF-domain ultrasound signal processing.

The basic internal mechanical forces acting on a blood vessel are induced by pressure and shear stress. These induced forces are related to the pulsatile flow of blood through the arteries. Pressure acts normal to the axis of the vessel wall while shear stress $\tau$ represents the tangential component of a force field acting on the vessel wall and is defined as:

$$
\tau=\left.\eta \cdot \frac{\partial v}{\partial r}\right|_{r=R}
$$

where $\eta$ is fluid viscosity, $d v / d r$ shear rate, $v$ the axial velocity, $r$ the radial coordinate and $R$ the radius of the blood vessel. Because blood is a non-Newtonian fluid, its viscosity is a non-linear function of shear rate (Fig. 5.1). From in vitro studies it is known that endothelial monolayers undergo a change in structure and function in response to an imposed shear stress (Caro et al. 1983, Nerem and Girard 1990, 
Helmlinger et al. 1991). It has also been demonstrated that wall shear stress has a significant influence on the production of biochemical mediators by endothelial cells (Davies 1988, Davies 1989, Davies and Barbee 1994). Consequently, it is believed that wall shear stress plays a key role as fluid mechanical mediator in vessel wall anomalies (Friedman et al. 1981, Friedman 1990, Nerem 1992, Fung and Liu 1993). However, the role of shear stress in vascular disorders is unknown, mainly because at present no noninvasive methods are available to assess wall shear rate in vivo. However, to compute shear stress from shear rate it is still necessary to determine the apparent viscosity $\eta$ which demands an invasive technique.

In vitro estimates of shear stress have been obtained using high resolution velocity profiles, recorded with laser Doppler anemometry (LDA) (Drain 1980). Ku et al (1987) performed LDA measurements in a rigid model of the carotid artery bifurcation. In these studies wall shear rate $\left(d v /\left.d r\right|_{r=R}\right)$ was actually estimated from the velocity profiles while shear stress was computed by means of equation (5.1) (Ku et al. 1985). However, these values are not necessarily representative for the in vivo situation.

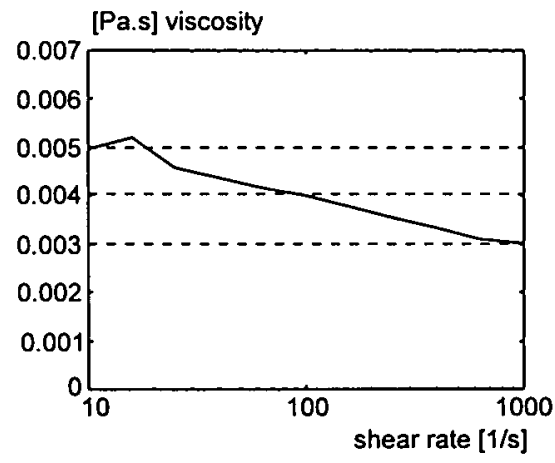

Figure 5.1: Measured relationship between shear rate and viscosity for human blood cells for the given volume concentration of the red blood cells ( $\mathrm{HT}=46)$.

The viscosity shear rate relationship in Fig. 5.1 of the fresh human blood was determined by means of a plate viscosimeter (Rheometrics RFS II) at a temperature of $37^{\circ} \mathrm{C}$.

This chapter presents a non-invasive method to assess in vivo wall shear rate as function of time from velocity profiles, recorded by means of received ultrasound RF-signals. The measurement system consists of an ultrasound echo system, a data acquisition system to store the ultrasound information received during one second, and a computer system to estimate off-line wall shear rate. The information in the acquisition memory is read off-line by a computer system and stored as a matrix organized in time and depth: the RF-matrix (Fig. 5.2). 
From the RF-matrix (Fig. 5.2) off-line signal processing reveals an estimate for vessel wall displacement and the instantaneous blood flow velocity distribution, both as function of time. From each velocity profile an estimate of wall shear rate is obtained from the locally determined maximum axial shear rate $|\mathrm{dv} / \mathrm{dr}|$ between the vessel axis and the near and far wall (Fig. 5.3).

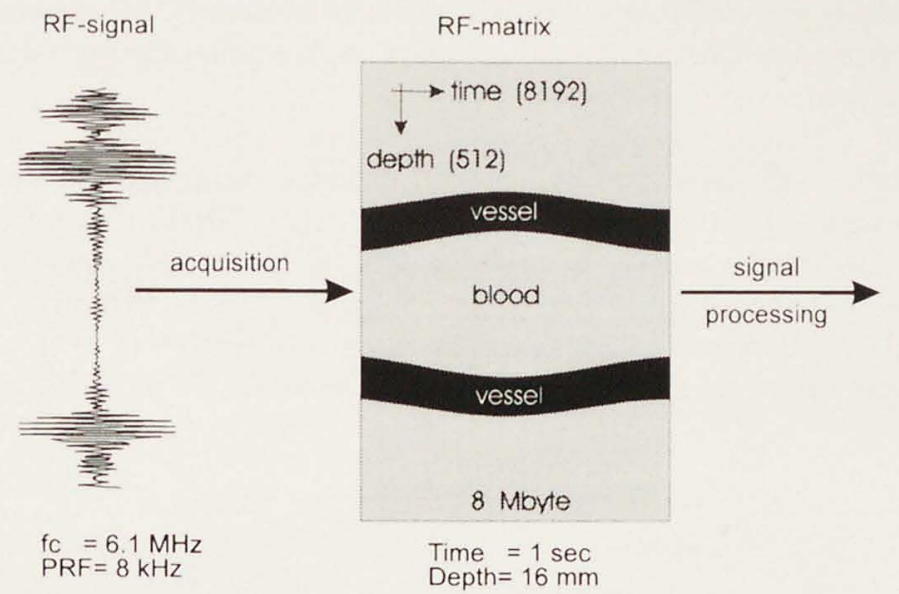

Figure 5.2: Data storage of the acquired RF-signals in an RF-matrix. RF-signals are sampled in depth (top to bottom) and time (left to right).

The sign of the estimated wall shear rate is obtained from the sign of the velocity profile at the position of the local maximum (far and near wall). Measurements in the femoral artery show indeed a change in sign of the estimated wall shear rate, but in the common carotid artery we have never been able to show a change in sign, when the velocities are recorded 2-3 $\mathrm{cm}$ proximal of the flow divider. The accuracy of the estimation method $(\mathrm{dv} / \mathrm{dr})_{\max }$ for wall shear rate depends strongly on the accuracy of the assessment of the near wall velocities. Due to the limited resolution of the ultrasound sample volume (SV) (part of the SV in the wall and a part in the blood vessel) averaging of the velocity estimate near the wall causes axial shear rate to decline as radius increases (Fig. 5.3).

To validate the method of wall shear rate assessment we investigated in a pilot study (6 repeated measurements in the carotid artery distributed over 5 healthy volunteers) the reproducibility of the assessment of peak wall shear rate as compared with that of peak center-line blood flow velocity (middle of the vessel). The common carotid artery was chosen for this pilot study because of: 1) its easy access to ultrasound;2) its straightness with only minor secondary velocity components. 


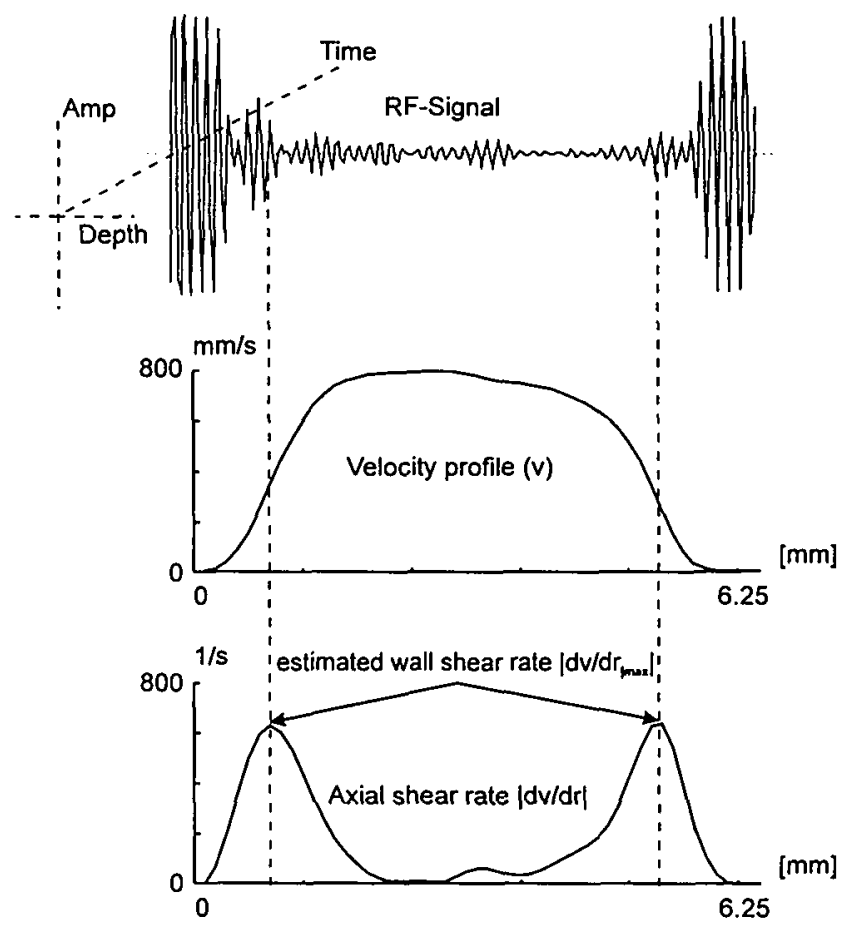

Figure 5.3: Example of the procedure for the estimation of wall shear rate from an assessed velocity profile by means of the maximum derivative of the observed spatial velocity distribution with respect to the radius $|\mathrm{dv} / \mathrm{dr}|_{\max } \mid$.

\subsection{Data acquisition}

The acquired RF-signals are processed off-line, offering the opportunity to test and implement new algorithms for signal processing. Another advantage of off-line processing is that the raw information (ultrasound information in the form of RFsignals) can be retained on tape so that all the data remain available for use of new algorithms. The measurement system consists of an echo system with an emission frequency of $6.1 \mathrm{MHz}$, a data acquisition system and a computer system (Fig. 5.4). The acquisition system samples and stores the RF-signals in real-time. It has a sample frequency (synchronously with the emission trigger of the attached echo system) with a maximum of $50 \mathrm{MHz}$, an acquisition memory of 4 Mword (1 word is 10 bit) and a dynamic range of $60 \mathrm{~dB}$ (10 bit). Data acquisition is enabled by a foot-switch and starts synchronously with a trigger derived from the R-wave of an ECG-signal. The acquisition of each RF-signal received starts after a selected delay with respect to the emission trigger, allowing free selection of the range of interest. 
The echo system is connected to the acquisition system by the following three signals: 1) the RF-signal after amplification and band pass filtering according to the quality factor of the ultrasound transducer used; 2) a trigger to indicate the moment of ultrasound transmission; 3) a sample clock synchronous with the emission trigger $\left(f_{s-}\right.$ $r_{f}=24.4 \mathrm{MHz}$ ). The latter is necessary to retain the phase information in the sampled RFsignals. Considering the quality factor $Q$ ( $Q$ =Mean frequency/Bandwidth) of the ultrasound transducer a sample frequency of three or four times the emission frequency is adequate to capture all the available signal information. The connection of the acquisition system to the computer system consists of a 12 bit parallel I/O interface with a handshake protocol. It takes about 2 minutes to transfer the 4 Mword (10 bit) of the acquisition memory and to store it on harddisk as 8 Mbyte.

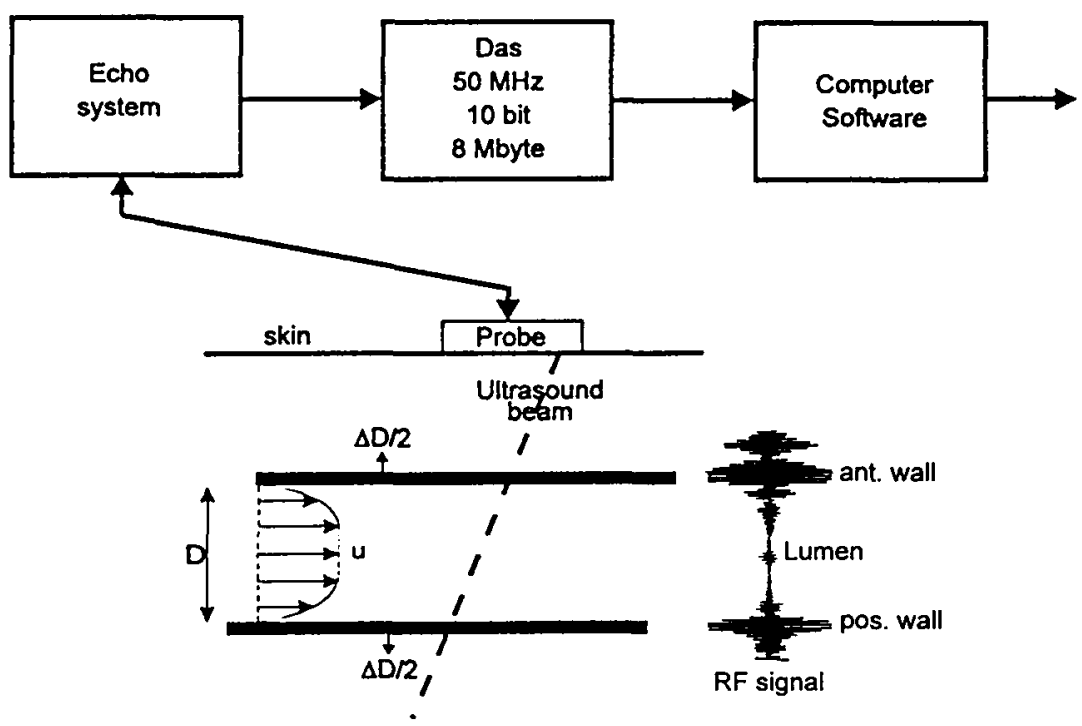

Figure 5.4: Block diagram of data acquisition consisting of an echo system, a data acquisition system (DAS) and a computer system.

On the harddisk of the computer system the raw temporal and spatial ultrasound information is stored in an RF-matrix (Fig. 5.2). The number of temporal and spatial sample points, i.e. the total amount of ultrasound information, is free as long as this product does not exceed the 4 Mword of the acquisition memory. The number of temporal and spatial sample points can only be varied in steps of 16 sample points, which is a reflection of the organization of the acquisition memory. Here we use the acquisition memory organized as 8192 temporal RF-signals each with 512 spatial sample points. The latter covers a depth range of $16 \mathrm{~mm}$ which is sufficient to investigate peripheral arteries like the carotid or femoral artery. For a pulse repetition frequency (PRF) of $8 \mathrm{kHz}, 8192$ temporal RF-signals are equivalent to $1 \mathrm{sec}$, covering at least one cardiac cycle. Therefore, the RF-matrix, which is the basic object for the 
off-line signal processing, consists of one second of temporal and $16 \mathrm{~mm}$ of spatial ultrasound information with a temporal sample interval of $125 \mu$ s (PRF: $8 \mathrm{kHz}$ ) and a spatial resolution of $250 \mu \mathrm{m}$ assuming $Q=2$.

Important is the dynamic range of the received RF-signals, being on the order of $72 \mathrm{~dB}$ therefore not fitting within the $60 \mathrm{~dB}$ dynamic range of the acquisition system. To solve this problem an additional amplification of $18 \mathrm{~dB}$ is applied. The latter will move up the noise level, while the upper $18 \mathrm{~dB}$ level is discarded by clipping at $0 \mathrm{~dB}$. This solution is possible because the information about blood flow velocity and vessel wall motion is retained in the phase of the RF-signals.

\subsection{Mean frequency estimation}

In the past decades there was a continuous evolution of mean frequency estimators for low frequency (LF) Doppler signals; more recently mean frequency estimators acting in the RF-domain have become available (Bonnefous and Pesque 1986) (Section 2.3). These estimators were developed because of the need for accurate estimation of mean frequencies in short temporal estimation windows in both the LF- and RF-domain. Exceptionally short estimation windows are used in Color Coded Doppler systems that display in real-time the two dimensional blood flow velocity distribution. These Color Coded Doppler systems are working with estimation windows on the order of 1 to 2 msec, corresponding to 8 or 16 temporal sample points, at a $P R F$ of $8 \mathrm{kHz}$ respectively. Restricting the velocity assessment to a single line of observation (M-mode) in assessing the velocity distribution as function of time, the length of the temporal estimation window can be extended. An temporal estimation window length of 8 to 10 ms ensures a low standard deviation of the velocity estimate while it does not compromise the temporal behavior (acceleration) of the observed velocities.

The quality of an temporal mean frequency estimator either in RF- or LF-domain strongly depends on the model used for the received ultrasound information (Section 2.2 and 2.4). From the single mean frequency estimators for the LF-domain the estimator based on the first order auto-regressive (AR) model (Kasai et al. 1985, Ahn and Park 1991) (Section 2.3.4) has the best performance to obtain an estimate of the temporal mean frequency if the estimation window length is greater than 16 sample points (Section 2.4.2). In the case of LF-domain processing, the spatial estimation window length is given by the length of the demodulation window which generally corresponds to the quality factor $Q$ of the ultrasound probe. Recent studies of the relation between the spatial estimation window length and the quality of an LF-domain estimator demonstrated that the quality of the estimate is almost independent of this spatial window length (Hoeks et al. 1993). However, better results can be obtained by using LF-domain spatial subsample processing (Hoeks et al. 1994). Another problem, related to LF-domain processing, is that the spatial mean frequency $\bar{f}_{c}(d)(1.1)$ used for demodulation is assumed to be constant over depth. Actually the emission frequency of the ultrasound transducer is used as mean spatial frequency $\bar{f}_{c}(d)$. However, due to 
frequency dependent attenuation the carrier frequency will be shifted down as function of depth. Consequently the velocity at greater depths is underestimated (1.1). To achieve a high spatial resolution short bursts of ultrasound should be emitted requiring a large bandwidth (low $Q$ ) of the system, causing a substantial bias in the estimated velocities. This problem can be avoided by employing an RF-domain temporal mean frequency estimator. The estimator with the best quality to obtain an estimate of mean blood flow velocity in the RF-domain in a given time and depth window is based on a Gaussian model of the spatial cross-power spectral density distribution of received ultrasound RF-signals (2.34). The RF-domain mean frequency estimator derived from this model (CCM; Section 2.3.8) is given by the following equation:

$$
\hat{\varphi}=\frac{\operatorname{atan} 2\{0.5(\hat{R}(1,1)-\hat{R}(-1,1)), \sin \{\operatorname{acos}(\hat{R}(1,0) / \hat{R}(0,0))\} \cdot \hat{R}(0,1)\}}{\operatorname{acos}(\hat{R}(1,0) \cdot \hat{R}(0,0))}
$$

where $\hat{R}(k, i)$ is the estimated correlation function from a given estimation window in the RF-matrix and $\hat{\varphi}$ the estimated dimensionless velocity (Section 2.3.8). The operator atan2 denotes a double argument arctangent operation. The cross-correlation function $R(k, i)$ is estimated using the data points within a rectangular estimation window of 8 sample points in depth (spatial estimation window length) and 64 sample points in time (temporal estimation window length). The estimated normalized velocity is related to (blood flow) velocity as:

$$
\hat{\bar{v}}=\frac{c}{2 \cdot \cos \alpha} \cdot \frac{P R F}{f_{s-r r}} \cdot \hat{\varphi}
$$

\subsection{Vessel wall filter}

The vessel wall filter necessary to suppress the reflections induced by stationary or slowly moving structures is an adaptive high-pass filter (Section 4.4.5). In this way the reflections are suppressed by a band stop filter that apparently adapts its central frequency to the mean frequency of the slowly moving vessel wall.

To shift the frequencies downward the real RF-matrix is converted to a complex RFmatrix by a Hilbert transformation (Fig. 3.8; Section 3.3.5) performed in the spatial domain as:

$$
u_{r}(k, i)=z_{r r}(k, i)+j \cdot \frac{2}{\pi} \sum_{h=1}^{N_{h}} \frac{z_{r f}(k-h, i)-z_{r f}(k+h, i)}{h} \quad h=1,3,5 \ldots N_{h}
$$

where $z_{r f}(k, i)$ is a sample of the RF-matrix (Section 2.3.1), $u_{r f}(k, i)$ a sample of the complex RF-matrix and $N_{h}$ the spatial Hilbert transformation window. The latter was 15 spatial sample points $(500 \mu \mathrm{m})$ and is reciprocally related to the quality factor $Q$ of the ultrasound transducer. 
The amplitude of the vessel wall reflections is on the order of $40 \mathrm{~dB}$ higher than that of the signals induced by blood. Since the vessel wall induces a single velocity the bandwidth is marginal and an as low as possible cut-off frequency can be selected. The temporal length of the estimation window $(8 \mathrm{msec}$ ) limits the cut-off frequency (Section 3.3.2) to $125 \mathrm{~Hz}\left(b_{0}=0.0842\right)$ which gives a suppression of wall artefacts of on the order of $40 \mathrm{~dB}$ for frequencies below $16 \mathrm{~Hz}$. The cut-off frequency of the high-pass filter sets also the minimum velocity that can be assessed adequately. Because of the adaptive nature of the vessel wall filter the minimum velocity that can be determined is considerably less (Fig. 4.10) than for a static filter where the cut-off frequency is specified by the maximum velocity of the vessel wall (Section 3.3.2).

The adaptive wall suppression algorithm involves the location of the temporal spectral peak $\hat{f}_{r}$ of a reflection (Fig. 4.3) which is estimated by means of the cross-correlation model (CCM, Section 2.3.8) estimator. An error in the estimate of the location of the spectral peak will occur if the power of the first spectral component (reflection) is low in comparison with the second spectral component (scattering). This situation is possible for reverberations originating from within the lumen of a blood vessel. The error in the estimate of the location of the spectral peak introduces a bias in the estimated mean temporal frequency $\hat{\bar{f}}_{r}$. Hence, the spectral peak of the reflection will not be shifted to zero frequency and as a consequence the high-pass filter will not suppress the power of a reflection adequately. Reflections originating from vessel walls and reverberations from the lumen have a difference in power of at least $20 \mathrm{~dB}$. Therefore, the estimated signal-to-noise ratio $S \hat{N} R_{r}(4.4)$ of the reflections with respect to the background (either the blood induced signals or noise) can provide information about the origin of a reflection. If for an estimation window $S \hat{N} R_{r}$ is less than $17 \mathrm{~dB}$ $\left(S \hat{N} R_{r}<50\right)$ or if the estimated dimensionless velocity $\hat{\bar{\varphi}}_{r}(5.3)$ corresponds with a velocity higher than $10 \mathrm{~mm} / \mathrm{s} \hat{\bar{f}}_{r}$ is set to zero, eliminating the adaptation process. The restriction for the maximum velocity of a structure is necessary to be able to omit possible false positive and false negative results of the SNR decision rule. By means of both decision rules only reflections from structures with a high $S \hat{N} R_{r}$ are filtered in an adaptive way and reflections with a low amplitude (lumen) are filtered in the static way. As a result, the adaptive vessel wall filter is only adaptive in a small spatial range around the vessel wall-lumen boundary.

It is important to realize that as a result of the adaptive shift of the mean temporal frequency of a reflection to zero frequency (Fig. 4.3), the dimensionless velocity estimator of equation (5.2) is modified as follows:

$$
\hat{\varphi}=\hat{\varphi}_{s}+\hat{\varphi}_{r}
$$

where $\hat{\varphi}_{r}$ is the estimated dimensionless velocity of a reflection and $\hat{\varphi}_{s}$ the estimated dimensionless velocity of the scattered signal. The estimated dimensionless velocity $\hat{\varphi}_{s}$ 
is corrected for the shift of the mean temporal frequency of the reflections to zero frequency (Fig. 4.3).

The resolution along the ultrasound beam is specified by the bandwidth of the ultrasound system where a larger bandwidth (low $Q$ ) provides a better axial resolution. Therefore, an echo system with a high emission frequency $(6.1 \mathrm{MHz})$ and a low quality factor $(Q=2)$ was used for the in vivo pilot study. The length of the spatial estimation window of the CCM estimator was set equal to the axial resolution of the ultrasound system, i.e., 8 sample points by a sample frequency of $24.4 \mathrm{MHz}(250 \mu \mathrm{m})$. The length of the temporal estimation window of the CCM estimator was set to $8 \mathrm{msec}$, i.e., 64 sample points at a PRF of $8 \mathrm{kHz}$. Due to the stochastic character of the ultrasound signals, the quality of the velocity estimate depends on the location of the estimation window within the RF-matrix. To improve the quality of the velocity estimates the RFmatrix is divided into estimation windows half overlapping, in both directions time and depth. It is obviously that only the depth segment covering the artery of interest is subjected to RF-domain signal processing. The selection of this depth segment is made by the user, based on the vessel wall reflections, using the first RF-line acquired. To cope with possible diameter variations and lumen displacements the depth range for processing is enlarged with two additional estimation windows on each side (extending into the anterior and posterior vessel wall).

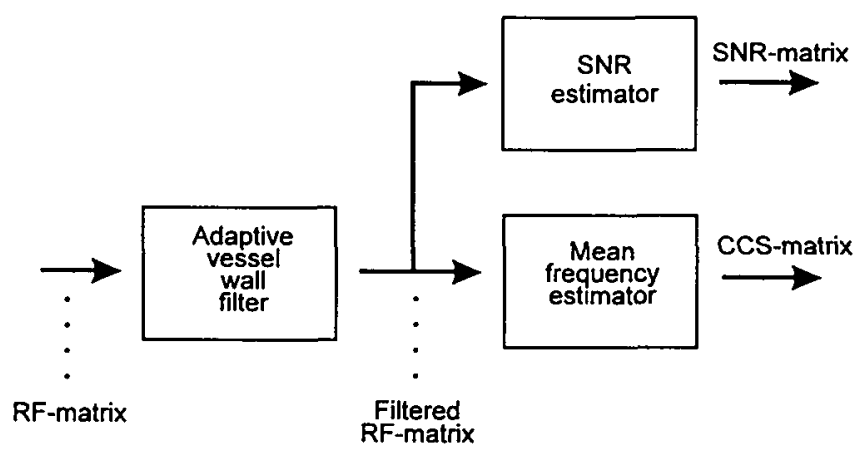

Figure 5.5: Block diagram of the RF-domain signal processing consisting of a mean frequency estimator (CCM) preceded by an adaptive vessel wall filter.

The total RF-domain signal processing (Fig. 5.5) to estimate the time dependent blood flow velocity distribution consists of:

1) Division of the RF-matrix into half overlapping estimation windows $(N, M)$ (Section 2.3.1) based on the identification of the vessel wall reflections,

2) Estimation of the temporal mean frequency $\hat{\bar{f}}_{r}$ and the $S \hat{N} R_{r}$ of the reflections for each of the estimation windows $(N, M)$,

3) Suppression of the reflections with an adaptive vessel wall filter resulting in a filtered RF-matrix, 
4) Estimation of the dimensionless velocity $\hat{\varphi}_{s}$ (CCS-matrix) together with the estimation of the $S \hat{N} R$, (SNR-matrix) of the scattered signals for each of the estimation windows $(N, M)$,

5) Post processing of the dimensionless velocity (CCS-matrix) based on information in the SNR-matrix (Fig. 5.6) and

6) Conversion of the CCS-matrix to blood flow velocity (5.3).

The result of the signal processing (Fig. 5.5) is a cross-correlation shift matrix (CCSmatrix, dimensionless velocity) and a signal-to-noise matrix (SNR-matrix) both based on estimates from the filtered RF-matrix.

To reduce the computational load and to eliminate the transient response of the highpass filter the first four steps of processing are performed in a temporal recursive way. Due to the half overlapping estimation windows the CCS-matrix is based on a temporal sampling interval of $4 \mathrm{msec}$ (half the temporal estimation window length) at a spatial sampling interval of $125 \mu \mathrm{m}$ (half the spatial estimation window length). Hence, the CCS-matrix consists of a total of 256 temporal sample points $(\approx 1 \mathrm{sec})$ and a number of spatial sample points depending on the actual blood vessel diameter, usually on the order of 48 to 64 sample points ( 6 to $8 \mathrm{~mm}$ ).

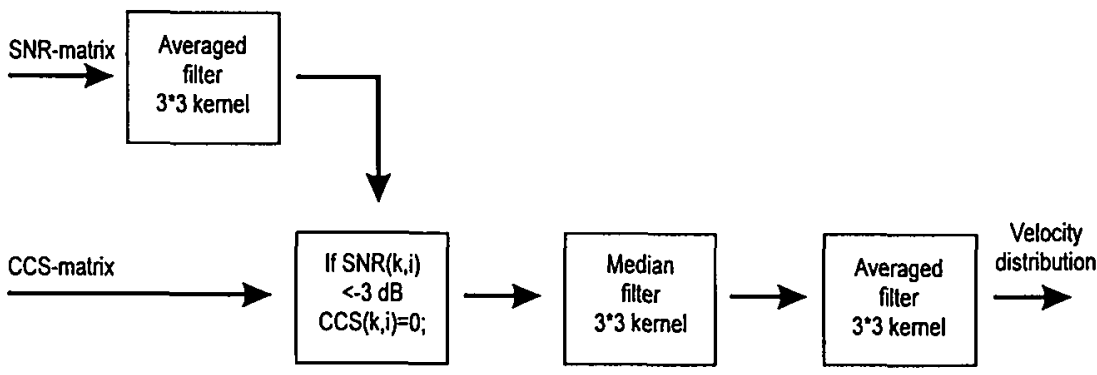

Figure 5.6: Block diagram of the post processing performed on the CCS-matrix with information from the SNR-matrix, yielding the velocity distribution over time.

Post-processing is performed to make the quality of the estimate independent of the position of the estimation window in the RF-matrix (Fig. 5.6). The SNR-matrix is subjected to an averaging filter with a $3 * 3$ kernel to reduce the variation of the $S \hat{N} R_{s}$. If for the given size of the estimation window ( 64 by 8 sample points) the smoothed SNR is less than $-3 \mathrm{~dB}\left(S \hat{N} R_{s}<0.5\right)$, it may be expected that the estimation window does not contain blood induced signals and the corresponding estimate for the dimensionless velocity $\hat{\varphi}_{s}$ is set to zero. The value of $-3 \mathrm{~dB}$ is an arbitrary compromise between the expected number of false positive and false negative results of the SNR decision rule against the quality of the velocity estimates for the slowly moving blood near the vessel wall. If the number of sample points within an estimation window is varied, the threshold should be adjusted accordingly. To remove possible false positive and false negative decisions, inducing spot noise, the CCS-matrix is subjected to a median filter 
with a $3 * 3$ kernel. Furthermore, the CCS-matrix is subjected to an averaging filter, also with a $3 * 3$ kernel, to remove the quantisation noise introduced by the median filter. Finally the CCS-matrix is converted to blood flow velocity.

In figure 5.7 the observed velocity profiles have a blunt appearance. This is illustrated by the intersects showing all the velocity profiles over a short time range in the phases of the cardiac cycle indicated.

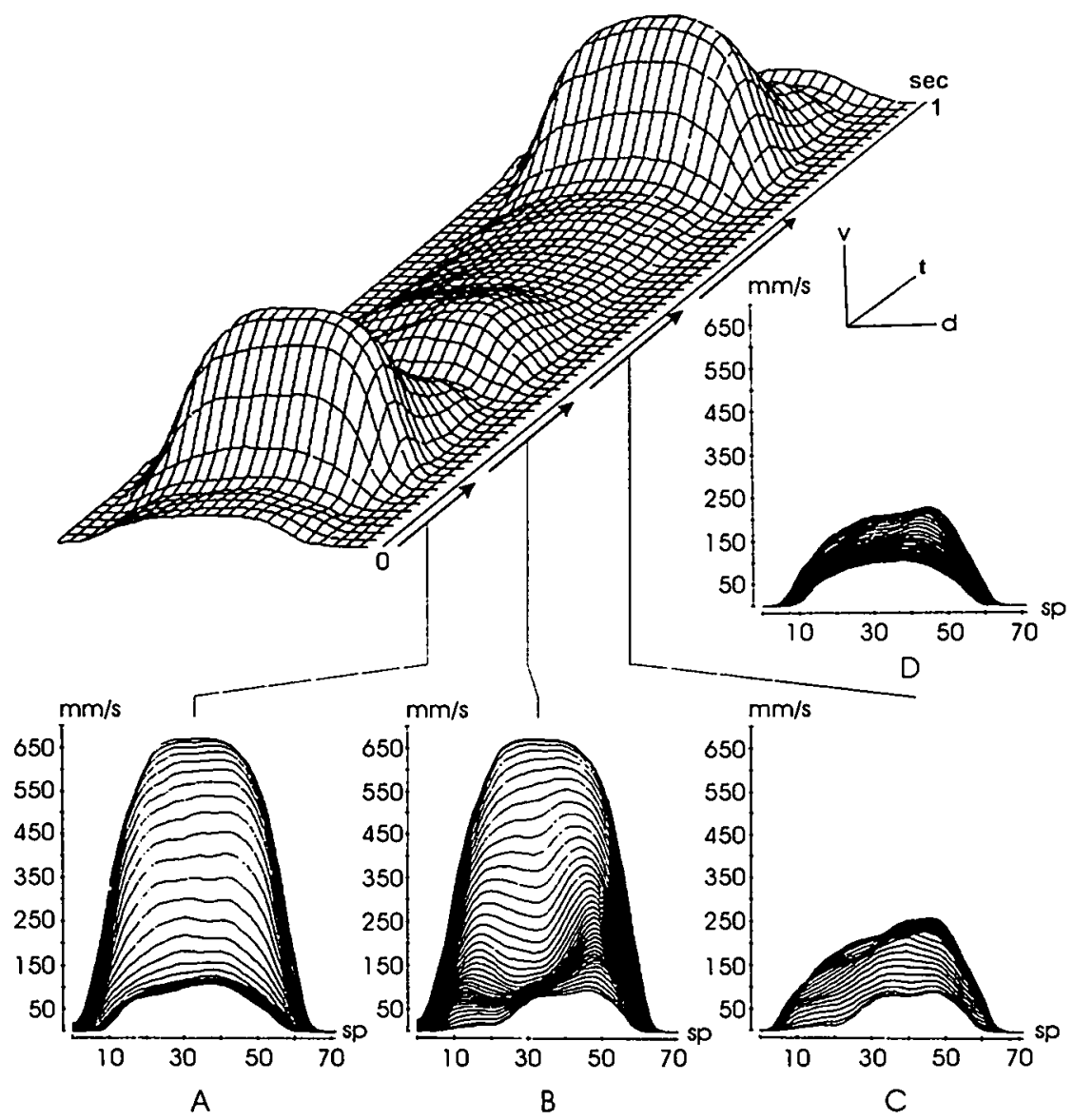

Fig. 5.7: The time-dependent blood flow velocity distribution as estimated from the raw ultrasound information measured in the common carotid artery (diameter $=6.5 \mathrm{~mm}$ ). Moreover, some blood flow velocity profiles as estimated at various regions in a heartbeat are shown (A: systole, B: late systole, $\mathrm{C}$ : mid diastole, $\mathrm{D}$ : late diastole). The unit used for the spatial axis is sample points (sp) and $\mathrm{I} s \mathrm{sp}$ is equal to $109 \mu \mathrm{m}$. 


\subsection{Wall shear rate estimation}

The spatial derivative of the velocity distribution (Fig. 5.8a) gives the shear rate distribution as function of depth and time (Fig. 5.8b).

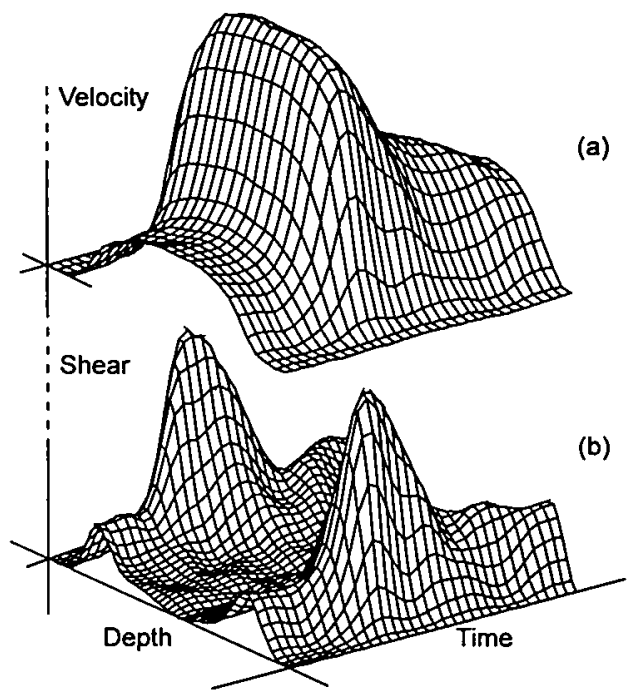

Figure 5.8: The time dependent shear rate distribution (b) as derived from the time dependent velocity distribution (a) by means of $|\mathrm{dv} / \mathrm{dr}|$ per blood flow velocity profile.

The maximum on both sides is considered to be an estimate of wall shear rate. The blood flow velocity in a blood vessel is a vector with an axial, a lateral and an azimuth component ( $x-, y-, z$-component) where the estimated velocity is a projection of the three vector components on the ultrasound beam. Assuming that the line of observation passes through the axis of the vessel, the azimuth-component may be ignored. The lateral component (radial direction) will act on the anterior and the posterior side of the lumen in opposite ways.

To estimate wall shear rate caused only by the axial blood flow velocity component, the wall shear rates of the anterior and the posterior vessel wall are averaged as:

$$
\hat{s}(t)=\frac{1}{2}\left\{\left|d v(r, t) / d r_{0}\right|_{\text {max }}|+| d v(r, t) /\left.d r_{1}\right|_{\text {max }} \mid\right\}
$$

where $\hat{\mathrm{s}}(\mathrm{t})$ is the estimated time dependent wall shear rate (Fig. 5.9), $v(r, t)$ the estimated blood flow velocity profile (Fig. 5.7 and 5.8) and $r_{0}$ and $r_{l}$ the radial positions measured from the vessel axis to the near and far walls respectively. 
To obtain information about the elasticity of the artery, the vessel wall displacement (distension) during the cardiac cycle is estimated using the CCM estimator together with the blood flow velocity distribution and wall shear rate. The estimate of vessel wall displacement is performed by means of two tracking estimation windows (Hoeks et al. 1990 ), positioned at the vessel wall reflections by the user. The tracking window dimension $(8 \mathrm{msec}$ by $250 \mu \mathrm{m})$ is the same as used for blood flow velocity estimation. The difference in estimated displacement observed in both tracking windows provides the time dependent distension of the blood vessel. The latter together with the time dependent center-line blood flow velocity and the time dependent wall shear are socalled circulation parameters: distension (Fig. 5.9a), center-line blood flow velocity (Fig. 5.9b) and wall shear rate (Fig. 5.9c). From these circulation parameters the minimal and maximal values, the difference between minimal and maximal values, the rise time in the systolic phase, and the mean of the signal over one cardiac cycle are derived. Moreover, from the minimal distension heart rate and the diastolic diameter are determined. These characteristics may be used for the characterization of the hemodynamic state of a blood vessel, to use for example in population studies.
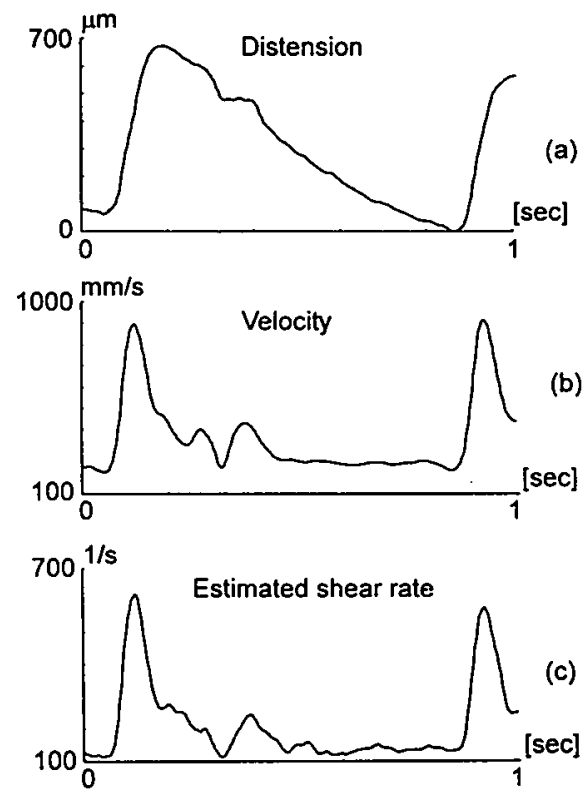

Figure 5.9: Example of circulation waveforms consisting of: (a) distension, (b) blood flow velocity in the middle of the vessel and (c) wall shear rate.

The validation of the method proposed to estimate wall shear rate was performed in an in vivo study. This in vivo study concerns a pilot study regarding the reproducibility of the assessment of peak wall shear rate as compared with that of peak center-line blood flow velocity. 
The in vivo reproducibility study was performed on five presumed healthy male volunteers between 18 and 34 years of age. Measurements were made six times in the right common carotid artery about $2 \mathrm{~cm}$ proximal to the tip of the flow divider. For all measurements the angle of observation was close to $60^{\circ}$ with the vessel axis in the plane of the bifurcation. The result of this pilot study is presented in table 5.1.

Table 5.1: Data from the pilot study (6 repeated measurements in the common carotid artery of 5 healthy volunteers) with in between parentheses the coefficient of variation as a percentage.

\begin{tabular}{|c|c|rr|rr|}
\hline $\mathrm{n}=6$ & $\mathrm{D} \mathrm{mm}$ & \multicolumn{2}{|c|}{$\mathrm{v}_{\mathrm{c}} \mathrm{mm} / \mathrm{s}$} & \multicolumn{2}{|c|}{ PSR s$^{-1}$} \\
\hline $\mathrm{A}$ & $6.55(4)$ & $1002(10)$ & 805 & $(2)$ \\
\hline $\mathrm{B}$ & $6.27(4)$ & $969(16)$ & 780 & $(8)$ \\
\hline $\mathrm{C}$ & $6.17(3)$ & $1157(11)$ & 982 & $(6)$ \\
\hline $\mathrm{D}$ & $6.15(2)$ & 962 & $(6)$ & 678 & $(4)$ \\
\hline $\mathrm{E}$ & $5.86(5)$ & 637 & $(4)$ & 393 & $(4)$ \\
\hline
\end{tabular}

In table 5.1 the end-diastolic diameter $D$, the peak center-line blood flow velocity $v_{c}$ and the peak wall shear rate $P S R$ are listed. The observed mean coefficients of variation of the peak center-line blood flow velocity was $9 \%$ while for the peak wall shear rate it was $5 \%$.

\subsection{Discussion}

As discussed in the introduction there is increasing evidence that wall shear rate and/or stress interacts functionally with the arterial wall and that these physical factors may play a role in the origin of vessel wall anomalies. The role of wall shear rate, however, is as yet unknown, mainly because no methods were available to assess non-invasively this physical factor in vivo. Therefore, we developed a non-invasive method to estimate wall shear rate in vivo from velocity profiles by means of the maximum derivative of the velocity with respect to the radius (Fig. 5.3). The velocity profiles were recorded by means of pulsed ultrasound. To determine wall shear rate from velocity profiles accurately, one must be able to assess unambiguously low blood flow velocities near the vessel wall. Inadequate spatial resolution is considered to be a major drawback of pulsed ultrasound because of the steep slope of the velocity profile near the vessel wall, which can give an underestimation of wall shear rate. An even more important limitation of pulsed ultrasound is its inability to discriminate unambiguously between signals induced by slowly moving structures, like vessel walls, and those induced by slowly moving blood near the vessel wall. To overcome this limitation we introduced a single mean frequency estimator (CCM, Section 2.3.8) preceded by an adaptive vessel wall filter (Section 4.4 .5 ) to determine accurately near-wall velocity behavior.

The estimated blood flow velocity is a projection of the blood flow velocity vector ( $\mathrm{x}$-, $y$-, z-component) on the ultrasound beam. The RF-signals received do not give any information about this projection. In practice, one assumes that the estimate is only based upon the axial blood flow velocity. This simplification is allowed because the 
standard deviation of the conventional estimators used is larger than the errors caused through blood flow velocity vector projection. Moreover, normally only blood flow velocity in the middle of a vessel is measured in a accurate way. In the middle of a relatively straight blood vessel the lateral and the azimuth component of the blood flow velocity vector are small in comparison with the axial blood flow velocity vector component. The lateral velocity components of the blood flow velocity vector near the vessel walls, which are mainly caused by the vessel wall movement, have an opposite direction. The contribution of the lateral velocity components is reduced by averaging the observed wall shear rates at the anterior and posterior walls (5.6). The above reasoning is only valid for a relatively straight vessel like the common carotid artery. Therefore, to obtain a reliable estimate of wall shear rate using the method proposed, the wall shear rate should be measured in relatively straight blood vessels.

The validation of the method proposed to estimate wall shear rate was performed in an in vivo study. This in vivo study concerns the reproducibility of peak wall shear rate assessment in the common carotid artery using 30 measurements in 5 healthy adult male volunteers. The relatively low coefficient of variation in the assessment of peak wall shear rate (average of near/far wall) over a wide range of peak wall shear rates, as compared to the other hemodynamic variable (velocity), suggests that our method allows a reproducible estimation of the peak wall shear rate and that the variation over time is considerably less than of the peak centre-line blood flow velocity.

\subsection{Conclusion}

In the present chapter a method is described to estimate non-invasively time dependent wall shear rate. This method is based upon the evaluation of the velocity distribution obtained by means of ultrasound using off-line signal processing. The off-line processing is performed in the RF-domain and consists of an RF-domain velocity estimator preceded by an adaptive vessel wall filter. The latter is adaptive to get an optimal discrimination between the slowly moving structures, like vessel walls, and the slowly moving blood near the vessel wall. The RF-domain velocity estimator is based on cross-correlation of RF-signals, using a Gaussian model of the cross-power spectral density distribution of RF-signals in depth. It can be concluded that the proposed method to estimate time dependent wall shear rate in vivo is reproducible. However, the method is only reproducible in relatively straight blood vessels because in curved vessels the wall shear rate estimates are affected by the value of the secondary velocities. The maximum spatial derivative of the blood velocity profile can give an underestimation of wall shear because of the limitation in spatial resolution of pulsed ultrasound. 


\section{Chapter 6}

\section{Validation of the method to estimate wall shear rate}

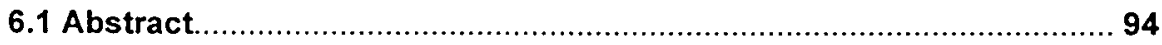

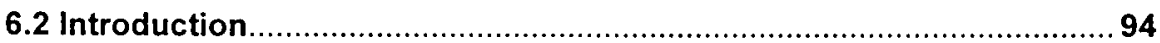

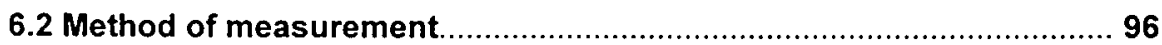

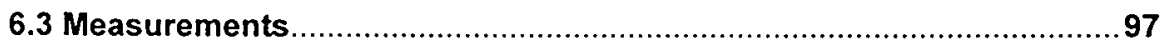

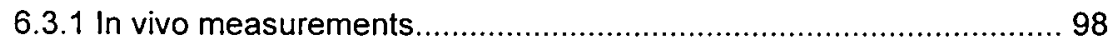

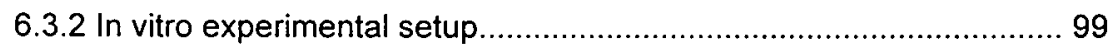

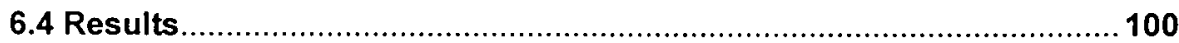

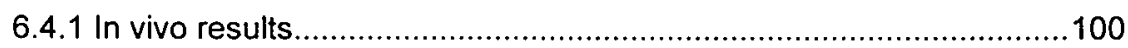

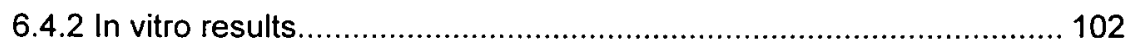

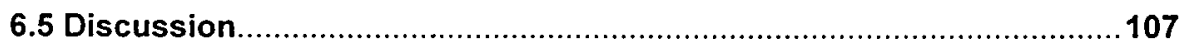

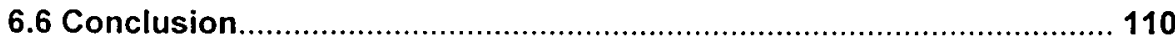

The contents of this chapter has partly been published in the article: Hoeks A.P.G., Samijo S.K., Brands P.J., Reneman R.S. Assessment of wall shear rate in humans: an ultrasound study. J. Vasc. Investigation, Vol. 1, No. 3, pp. 108-117, 1995. 


\subsection{Abstract}

It can be inferred from models on fluid transport that the shear stress exerted on the wall by the flowing blood should be on the order of $1.5 \mathrm{~Pa}$. Wall shear stress equals wall shear rate times local viscosity. The concept of constant mean shear stress implies an autoregulatory interaction between mean volume flow in an artery and the mean diameter of that artery. In vivo studies on the relation between diameter and shear rate in humans are scarce, mainly because methods to assess non-invasively wall shear rate are lacking. In this Chapter the in vivo and in vitro validation of the method as described in Chapter 5 to assess non-invasively wall shear rate in the human carotid artery is presented. The in vivo study concerns the reproducibility of mean wall shear rate in two subject populations of different age ( $20-30$ years, $n=9 ; 60-70$ years, $n=7)$. In agreement with the assumed autoregulation of wall shear stress, both categories exhibited the same mean wall shear stress of $1.2 \mathrm{~Pa}$ (shear rate of $340 \mathrm{~s}^{-1}$ ). The mean coefficient of variation for repeated measurements of mean wall shear rate was $13 \%$ in both populations, indicating a good level of reproducibility. The in vitro study concerns a comparison of the velocity distribution and wall shear rate assessed both with ultrasound and laser Doppler anemometry (LDA) in a distensible tube under nonstationary flow conditions, resembling the flow pattern in the descending aorta. The mean normalized difference in wall shear rate between the two methods was $9.4 \%$.

Key Words: carotid artery, laser Doppler anemometry, shear rate, ultrasound, velocity estimation, wall-blood interaction.

\subsection{Introduction}

In this chapter an in vivo and in vitro validation study of the method to estimate noninvasively wall shear rate using RF-domain ultrasound signal processing as presented in Chapter 5 is described.

From a minimum work load model it can be inferred that the mean shear stress at the wall, i.e., the stress exerted by flowing blood on the endothelial layer, has an optimal value and is maintained at that level irrespective of the mean hemodynamic conditions (Kamiya et al. 1984, Zarins et al. 1987, LaBarbera 1990, Zamir et al. 1992). Shear stress makes the endothelial cells to orient with the prevailing flow direction (Flaherty et al. 1972, Nerem and Girard 1990), induces the production of prostacyclin (Frangos et al. 1985) and activates $\mathrm{K}+$ channels (Olesen et al. 1988). The interaction between shear stress and artery diameter may be explained by the augmented or depressed release of endothelium-derived relaxing factor (EDRF) at shear rate levels deviating from base line level (Rubanyi et al. 1990). At high wall shear rate the released EDRF may induce an increase in artery diameter which, for the same volume flow, converts into a lower wall shear rate (Rodbard 1975, Zarins et al. 1987). 
Most of the evidence for the relevance of shear stress for endothelial cell function and structure has been obtained in cultured cells subjected to some constant shear stress. These data are supported by pathologic-anatomical examinations of the wall structure of excised vessel segments (Glagov et al. 1995). However, in vivo validation is scarce (Kamiya and Togawa 1980, White et al. 1994) and pertains to animal studies because of the limited possibility to assess non-invasively local shear stress or shear rate.

Since direct assessment of wall shear stress in vivo is difficult, this parameter is generally derived from wall shear rate. Assuming constant viscosity $\eta$ across the crosssection of the artery lumen local wall shear stress $\tau$ can be given in terms of wall shear rate $\gamma$ :

$$
\tau=\eta \cdot \gamma
$$

where

$$
\gamma=\left.\frac{\partial v(r)}{\partial \mathrm{T}}\right|_{r=\mathrm{R}}
$$

with $v(r)$ the velocity distribution as function of the radial position $r$ and $R$ the local radius of the artery. It should be noted that blood is a non-Newtonian fluid, so that its viscosity is a non-linear function of shear rate (Fig. 5.1). However, in the shear rate range between $100 \mathrm{~s}^{-1}$ and $1000 \mathrm{~s}^{-1}$ it is acceptable to assume a constant viscosity ( $\eta=3.5$ mPa.s).

The most frequently reported methods in literature to estimate wall shear rate (Kamiya and Togawa 1980, Kamiya et al. 1984, Zarins et al. 1987, White et al. 1994) are derived from a modeled velocity profile. For a circular shaped lumen a symmetric velocity profile may be modeled as:

$$
v(r)=v_{\max }\left\{1-\left(\frac{r}{R}\right)^{n}\right\}
$$

where $v_{\max }$ is the maximum velocity on the center of the vessel and $n$ the bluntness factor of the velocity profile. From expression (6.3) and the assumption of a circular shaped lumen wall shear rate can be given in terms of flow $Q$, bluntness factor $n$, maximum center velocity $v_{\max }$ and diameter $D$ :

$$
\gamma=\frac{2 \cdot n \cdot v_{\max }}{D}=\frac{8 \cdot(n+2) \cdot Q}{\pi \cdot D^{3}}
$$

It is obvious that the above mentioned expressions (6.4) are very sensitive to slight variations in the estimated radius and in the bluntness factor. The approach as described in Chapter 5, based on direct non-invasive assessment of the radial velocity gradient at 
the wall, does not require any assumptions about the shape of the velocity distribution except for rotational symmetry (straight artery).

An important restriction to estimate wall shear rate by means of pulsed ultrasound is caused by the inability of static clutter filters (Section 3.3.5) to discriminate unambiguously between signals induced by slowly moving structures, like vessel walls, and those induced by slowly moving blood near the vessel wall. To overcome this limitation we introduced an adaptive clutter filter (Section 4.4.5). Another important restriction to estimate wall shear rate is the maximum resolution along the ultrasound beam that can be obtained.

In this Chapter the method to estimate wall shear rate as described in Chapter 5 (Section 5.6) will be applied to velocity distributions as assessed with ultrasound and laser Doppler anemometry (LDA) in a flow model with an non-stationary flow pattern. Moreover, in an in vivo study the reproducibility of the assessment of mean wall shear rate with the method is investigated in two subject populations of different age.

\subsection{Method of measurement}

Conventional echo systems do not provide a pulse repetition frequency (PRF) of ultrasound bursts in echo $\mathrm{M}$-mode in accordance with the anticipated blood flow velocities. Only in pulsed Doppler mode the PRF can be set according to the depth of interrogation, but then the system switches to an unacceptable low axial resolution (long emission bursts, reduced receiver bandwidth). The practical limitations of commercially available echo systems are circumvented by an additional emitter/receiver unit, taking over control of the ultrasound probe once the ultrasound beam has been positioned correctly. The additional emitter/receiver provides an output of the RF-signal in combination with a reference clock synchronously with the trigger for emission. After completion of the data acquisition the acquired RF-signals are transferred to the memory of a personal computer and stored as an RF-matrix (Fig. 5.2). A preliminary check of the data integrity is executed whereafter the RF-matrix is stored on DAT (digital tape) for further off-line analysis.

The restricted size of the acquisition memory ( 4 Mword of 10 bit), in combination with a high sample frequency (20 $\mathrm{MHz}$ for an emission frequency of $5 \mathrm{MHz}$ ), and a high PRF necessitate careful selection of the length and position of the segment for conversion. For the in vivo study the RF-matrix was organized as 8192 temporal RFsignals each with 512 spatial sample points. For the in vitro study the RF-matrix was organized as 6144 temporal RF-signals each with 768 spatial sample points. The longer spatial window was necessary to cover the diameter of the tube $(20 \mathrm{~mm})$ in the in vitro flow model. Therefore, the RF-matrix consists of $1.4 \mathrm{sec}$ of temporal and $19 \mathrm{~mm}$ of spatial ultrasound information in the in vivo study and of $1.4 \mathrm{sec}$ of temporal and 29 $\mathrm{mm}$ of spatial ultrasound information in the in vitro study. In the in vivo study a PRF of 
$6 \mathrm{kHz}$ was used while in the in vitro study a PRF of $4.5 \mathrm{kHz}$ was used. Both studies were performed at an emission frequency of $5 \mathrm{MHz}$.

The spatial estimation window (Fig. 2.5, Section 2.3.1) of the RF-domain crosscorrelation model (CCM) estimator (Section 2.3.8) was selected according to the length of the emitted ultrasound burst in echo mode. For a $5 \mathrm{MHz}$ system with a quality factor of 2 sampled at $20 \mathrm{MHz}$ this converts to a depth window of 8 sample points, equivalent to a window of about $0.3 \mathrm{~mm}$. The temporal length of the estimation window (Fig. 2.5, Section 2.3.1) of the CCM estimator was selected according to the anticipated timedependent change in velocity characteristics and was set at $10 \mathrm{~ms}$, equivalent to 64 sample points (sp) at a pulse repetition frequency of $6 \mathrm{kHz}$ and $48 \mathrm{sp}$ at a pulse repetion frequency of $4.5 \mathrm{kHz}$. To retain smooth local changes in the estimated two-dimensional velocity distribution adjacent velocity estimates were based on sub-segments overlapping for $50 \%$ in both directions. This will give velocity estimates spaced at 0.15 $\mathrm{mm}$ along the ultrasound beam and $5 \mathrm{~ms}$ in time.

The time-dependent shear rate distribution directly follows from the observed velocity distribution by taking the radial derivative (expression 5.6, Fig. 5.8). For each observed velocity distribution (spaced at $5 \mathrm{~ms}$ ) the peak shear rate values at either wall-lumen transition is considered to be an estimate of local wall shear rate. The position of the wall-lumen interface is identified manually by placing sample volumes at correct positions on the first RF-line of observation. Since the acquisition starts synchronously with the R-top of the ECG the distance between both sample volumes reflects the enddiastolic internal diameter of the artery under investigation. Employing the $\mathrm{CCM}$ method the displacement waveform of the structures within these sample windows is estimated whereby the position of the sample windows is continuously adjusted (tracking windows). The difference between both waveforms reflects the change in lumen diameter over time (distension) (Hoeks 1993).

A major difference between the processing protocol, as outlined above, and the protocol reported in Section 5.5 is in the use of a smoothing filter after the median filter for the observed velocity distribution (Fig. 5.6). A smoothing filter will reduce the variance of the velocity estimate but only in regions where the velocity can be assumed to be constant. Evidently this requirement is not met close to the wall and for the present study the smoothing filter is deleted. Instead a median filter with a length of $26 \mathrm{~ms}$ is applied to the shear rate waveforms at both walls to reduce transient peaks due to a change in depth. Moreover, the region for peak shear rate evaluation has been reduced to 6 velocity points in depth $(0.9 \mathrm{~mm})$ rather than to $50 \%$ of the observed artery diameter. 


\subsection{Measurements}

\subsubsection{In vivo measurements}

Two groups of male, presumed healthy, volunteers were recruited. Group I consisted of 20 to 30 years old volunteers $(\mathrm{N}=9)$ and group II of $60-70$ years old volunteers $(\mathrm{N}=7)$. Both groups were normotensive and had no evidence of cardiovascular disease. From all volunteers written informed consent was obtained. The research protocol was approved by the joint ethical committee of the University Hospital Maastricht and the University of Limburg.

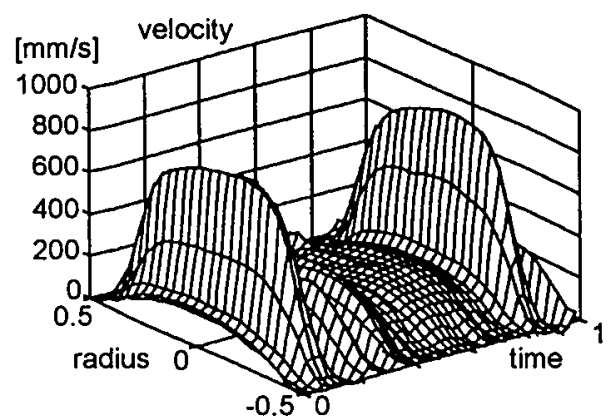

(A)

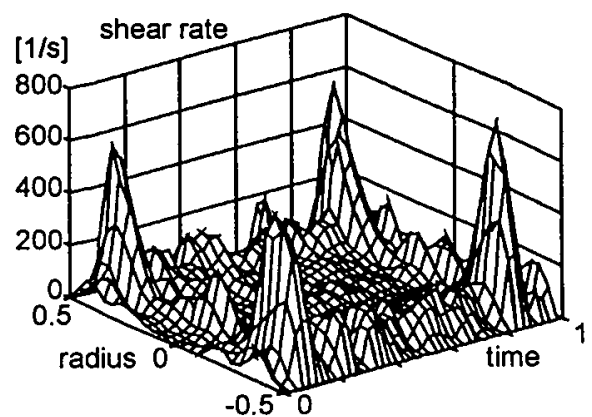

(B)

Figure 6.1: (A) Time-dependent velocity distribution observed in the common carotid artery of a young, presumably healthy, volunteer. (B) Time dependent shear rate distribution derived from the velocity distribution depicted in (A).

Data collection started after an adaptation period of 10 to 15 minutes in a supine resting position in an acclimatized room $\left(22^{\circ} \mathrm{C}\right)$. The common carotid artery was visualized in B-mode (ATL Mark IV, $5 \mathrm{MHz}$ mechanical sector scanner, Advanced Technology Labs, Bothell, WA, USA) with the head tilted in the contralateral direction. Using the tip of the flow divider as a landmark the probe position was manipulated until a suitable line of sight could be selected. It had to cross the carotid artery at an angle of 60 or 70 degrees, 2 to $3 \mathrm{~cm}$ proximal of the tip of the flow divider. After positioning the M-line the ultrasound system was switched to M-mode and a pulsed Doppler registration with the sample volume located midstream was made. The observed peak systolic velocity was noted. Subsequently the external emitter/receiver was activated and an RF registration of $1.4 \mathrm{sec}$ was acquired, where acquisition commenced synchronously with a trigger derived from the R-top of the ECG. The registration was random with respect to the phase of the respiration cycle. Before accepting a measurement a quick check of the acquired data was executed. A basic requirement for acceptance was agreement of the peak systolic center stream velocities obtained with the conventional pulsed Doppler system and with the shear rate system. Because the former system gives the spectral envelope and the latter system the mean velocity, it was acceptable that the latter system produced a lower mean velocity but it should not be less than $80 \%$ of the value obtained 
with the ATL system. The additional requirement was a consistent and corresponding behavior of the shear rate and velocity waveforms obtained with the latter system. Based on the criteria listed above on the order of $30 \%$ of the registrations had to be excluded because of excessive $R F$ interference, motion artefacts (movements of the artery with respect to the probe), or an inadequate SNR.

While the data were checked for velocity consistency the ultrasound probe was removed from the subject. Therefore, a subsequent registration for reproducibility measurements required exact replacement of the probe in the same position and orientation ${ }^{\prime}$.

\subsubsection{In vitro experimental setup}

The validation of the method to estimate wall shear rate (Chapter 5) was performed through an in vitro study in a flow model with a distensible tube with non-stationary flow conditions. The in vitro experiments were done at the department of Mechanical Engineering (WFW) of the Eindhoven University of Technology.

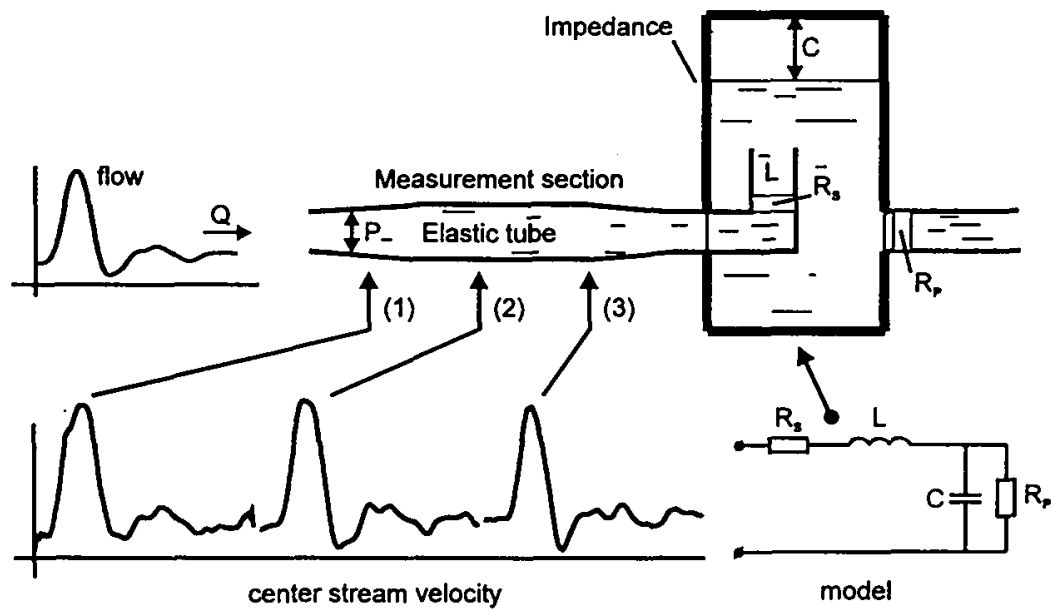

Figure 6.2: Schematic diagram of the in vitro setup, consisting of a distensible tube terminated with a hydraulic load.

The distensible tube in the in vitro setup (Fig. 6.2) was composed of two component silicon rubber and had a length of $545 \mathrm{~mm}$, an inner radius of $9 \mathrm{~mm}$, a wall thickness of $1 \mathrm{~mm}$ and a speed of sound of $1010 \mathrm{~m} / \mathrm{s}$. The tube was installed in a closed flow circuit with a stationary and a non-stationary pump. The stationary pump led the fluid from a reservoir to the distensible tube via a rigid inlet tube with a length of $3.75 \mathrm{~m}$ (200 times the tube diameter). The length of this rigid inlet tube was long enough to obtain a fully developed velocity profile at the entrance of the distensible tube. A non-stationary pump

\footnotetext{
${ }^{1}$ All the examinations were made by the second author (Steven Samijo).
} 
was installed between the stationary pump and the rigid inlet tube. The pulsatile pump generated a volume flow pulse $\left(f_{0}=1 \mathrm{~Hz}\right)$ based on the first ten harmonics of an aorta volume flow pulse presented by Milnor (Milnor 1989). To create physiological pressure-flow relations in the distensible tube, the tube was terminated with a hydraulic load (Fig. 6.2) characteristic for the thoracic aorta. The fluid in the flow circuit was a water-glycerol mixture ( $60 \%$ water and $40 \%$ glycerol) containing small air bubbles (10$30 \mu \mathrm{m}$ diameters) for ultrasound scattering. The fluid was Newtonian with a density of $1096 \mathrm{~kg} / \mathrm{m}^{3}$, a speed of sound of $1700 \mathrm{~m} / \mathrm{s}$ and a dynamic viscosity of $3.4 \mathrm{mPa}$.s. Distension of the tube and flow velocity distribution inside the tube were measured at three positions $(1=106 \mathrm{~mm}, 2=231 \mathrm{~mm}$ and $3=356 \mathrm{~mm}$ downstream of the entrance of the tube) with pulsed ultrasound and LDA. The flows proximal and distal to the tube were measured with electromagnetic flow probes (Skalar) providing volume flow. The pressure in the tube was measured using a catheter-tip pressure transducer (Millar) at the three given positions simultaneously with distension.

In the in vitro flow model the mean input flow and pressure over one pump cycle was 3 $1 / \mathrm{min}$ and $13 \mathrm{kPa}$, respectively. The peak to peak distension of the tube was $700 \mu \mathrm{m}$ (strain $3.5 \%$ ), the averaged flow velocity was $235 \mathrm{~mm} / \mathrm{s}$ and the mean peak wall shear rate was $661 \mathrm{~s}^{-1}$.

\subsection{Results}

\subsubsection{In vivo results}

Table 6.1 and 6.2 contain the number of accepted beats with respect to the number of acquired beats (second column), the end-diastolic diameter $D$, the peak to peak change in artery diameter during the cardiac cycle (distension Dist), the peak systolic velocity $P S V$ an the mean velocity over the cardiac cycle $M V$ in the center of the vessel, peak shear rate $P S R$ and mean wall shear rate WSR over the cardiac cycle.

Table 6.1: Individual results for the right common carotid artery for the younger age category (20-30y) with in between parentheses the coefficient of variation (CV) as a percentage.

\begin{tabular}{rrrrrrrr} 
Subject & beats & D [mm] & Dist [ $\mu \mathrm{m}]$ & PSV $[\mathrm{mm} / \mathrm{s}]$ & MV $[\mathrm{mm} / \mathrm{s}]$ & PSR [1/s] & WSR [1/s] \\
\hline jw67 & $11 / 20$ & $6.2(3)$ & $521(18)$ & $902(7)$ & $251(9)$ & $1088(25)$ & $338(19)$ \\
fa68 & $6 / 10$ & $6.3(5)$ & $436(11)$ & $790(16)$ & $299(14)$ & $763(24)$ & $294(13)$ \\
rc68 & $20 / 20$ & $7.0(3)$ & $640(9)$ & $917(7)$ & $298(38)$ & $902(18)$ & $296(8)$ \\
sw73 & $16 / 20$ & $7.2(3)$ & $908(8)$ & $1129(10)$ & $335(24)$ & $1489(14)$ & $420(15)$ \\
mb67 & $16 / 20$ & $6.3(3)$ & $534(16)$ & $852(8)$ & $263(12)$ & $1043(18)$ & $324(12)$ \\
Ik69 & $10 / 20$ & $6.0(4)$ & $610(20)$ & $930(4)$ & $284(16)$ & $1085(19)$ & $290(11)$ \\
pd72 & $10 / 20$ & $7.0(3)$ & $852(11)$ & $950(3)$ & $270(8)$ & $1149(9)$ & $335(12)$ \\
rb74 & $17 / 18$ & $6.5(4)$ & $735(17)$ & $1092(6)$ & $335(10)$ & $1424(9)$ & $408(14)$ \\
sk59 & $8 / 10$ & $6.1(5)$ & $405(12)$ & $961(8)$ & $359(10)$ & $993(21)$ & $369(12)$ \\
\hline Mean & & $6.5(3)$ & $627(13)$ & $947(7)$ & $299(18)$ & $1104(17)$ & $342(13)$ \\
SD & & $0.4(0.9)$ & $176(4.2)$ & $107(3.8)$ & $37(9.7)$ & $231(5.8)$ & $48(3.0)$
\end{tabular}


The numerical in the subject identification column of table 6.1 and 6.2 pertains to the year of birth. For some subjects 20 recordings, i.e., 20 heart beats have been considered while for others the total number was only 10 . As can be deduced from the second column of a total of 158 heart beats only 114 were accepted. The internal end-diastolic diameter could be assessed with a low coefficient of variation $\mathrm{CV}$, ranging from $3 \%$ to $5 \%$, and exhibits only a minor variation over the subjects (range 6.1-7.2 $\mathrm{mm}$ ). The peakto-peak change in artery diameter over a cardiac cycle (Dist) shows a more pronounced subject-to-subject variation while the CV ranges from $5 \%$ to $20 \%$. The peak systolic velocity $P S V$ was more consistent with a range from 790 to $1129 \mathrm{~mm} / \mathrm{s}$ but the velocity averaged over a cardiac cycle $(M V)$ had for some subjects an extremely high coefficient of variation. The observed peak shear rate PSR ranged from 763 to $1489 \mathrm{~s}^{-1}$ with a coefficient of variation varying between $9 \%$ and $25 \%$. For the mean wall shear rate WSR the subject-to-subject variation was high (range $294-420 \mathrm{~s}^{-1}, \mathrm{CV}: 8-19 \%$ ).

The wall shear rate $W S R$, averaged over all subjects, is $342 \mathrm{~s}^{-1}$ with a mean coefficient of variation of $13 \%$ (corrected for the observed beats per subject).

Table 6.2: Individual results for the right common carotid artery for the older age category (60$70 \mathrm{y}$.

\begin{tabular}{rrrrrrrr} 
Subject & beats & D [mm] & Dist [ $\mu \mathrm{m}]$ & PSV [mm/s] & MV [mm/s] & PSR [1/s] & WSR [1/s] \\
\hline cp30 & $13 / 20$ & $7.9(3)$ & $403(22)$ & $691(12)$ & $226(25)$ & $876(17)$ & $282(12)$ \\
wr33 & $3 / 20$ & $7.5(1)$ & $316(25)$ & $629(12)$ & $260(34)$ & $918(3)$ & $408(11)$ \\
gv27 & $5 / 20$ & $9.2(3)$ & $349(19)$ & $329(16)$ & $65(15)$ & $518(11)$ & $172(12)$ \\
pb26 & $8 / 20$ & $7.2(2)$ & $468(14)$ & $468(8)$ & $196(22)$ & $649(16)$ & $313(9)$ \\
pb18 & $5 / 10$ & $7.0(5)$ & $374(34)$ & $633(16)$ & $160(38)$ & $809(8)$ & $267(21)$ \\
wg33 & $11 / 20$ & $7.8(3)$ & $363(20)$ & $596(8)$ & $230(18)$ & $773(18)$ & $348(12)$ \\
nh28 & $8 / 10$ & $7.3(4)$ & $382(13)$ & $628(12)$ & $225(30)$ & $814(18)$ & $377(13)$ \\
\hline Mean & & $7.7(3)$ & $379(20)$ & $568(11)$ & $195(25)$ & $765(15)$ & $310(13)$ \\
SD & & $0.7(1.3)$ & $48(7.1)$ & $126(3.3)$ & $65(8.4)$ & $138(5.8)$ & $79(3.8)$
\end{tabular}

The older age category (60-70y) had a significant larger end-diastolic diameter than the young volunteers, ranging from 7.0 to $9.2 \mathrm{~mm}$ (CV:1-5\%), but the peak-to-peak distension was less (range $316-468 \mu \mathrm{m}, \mathrm{CV}: 14-34 \%$ ). This applied to the peak systolic velocity $P S V$ with a range from 329 to $691 \mathrm{~mm} / \mathrm{s}(\mathrm{CV}: 8-16 \%)$ and the mean velocity (range $65-260 \mathrm{~mm} / \mathrm{s}, \mathrm{CV}: 15-38 \%$ ) as well. One volunteer (gv27) had an exceptionally low mean center velocity of $65 \mathrm{~mm} / \mathrm{s}$ but quite a large end-diastolic diameter. The observed peak shear rate ranged from 518 to 918 per second with a coefficient of variation ranging from 3 to $18 \%$. Finally the mean wall shear rate ranged from 172 to 408 per second with a coefficient of variation varying between 9 and $21 \%$. The extreme low wall shear rate value was observed for the volunteer having a low mean velocity. Table 6.3 provides a direct comparison between both subject populations. Mean diameter increases with age from 6.5 to $7.7 \mathrm{~mm}$ while mean distension decreases from 627 to $379 \mu \mathrm{m}$. Likewise mean peak systolic velocity, the average mean velocity and peak systolic wall shear rate of the older age category is about $30 \%$ lower than that of the younger age group. However, the difference in mean wall shear rate between both groups is substantially less (young age group $342 \mathrm{~s}^{-1}$, standard deviation $48 \mathrm{~s}^{-1}$; old age group $310 \mathrm{~s}^{-1}$, standard deviation $79 \mathrm{~s}^{-1}$ ). Recalculating the mean value of $W S R$ in the 
older age category, excluding one outliner (gv27), gives a mean WSR of $333 \mathrm{~s}^{-1}$, which is not significantly different from the mean WSR in the younger age category.

Table 6.3: Summary of the results for the right common carotid artery of both populations (young and old).

\begin{tabular}{llrrrr} 
& & \multicolumn{2}{r}{ Young $(20-30 y), N=9$} & \multicolumn{2}{c}{ Old (60-70y), N=7 } \\
\hline & Quantity & Value & CV [\%] & Value & CV [\%] \\
\hline D & {$[\mathrm{mm}]$} & 6.52 & 3 & 7.70 & 3 \\
Dist & {$[\mu \mathrm{m}]$} & 627 & 13 & 379 & 20 \\
PSV & {$[\mathrm{mm} / \mathrm{s}]$} & 947 & 7 & 568 & 11 \\
MV & {$[\mathrm{mm} / \mathrm{s}]$} & 299 & 18 & 195 & 25 \\
PSR & {$[1 / \mathrm{s}]$} & 1104 & 17 & 765 & 15 \\
WSR [1/s] & 342 & 13 & 310 & 13
\end{tabular}

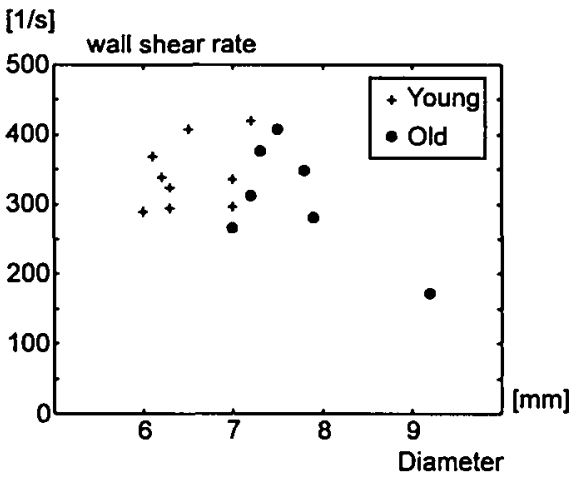

Figure 6.3: Scatter diagram of the observed mean wall shear rate versus observed end-diastolic diameter for both age groups.

Except for one extreme value in Fig. 6.3 the observed range of wall shear rates is the same for both age groups despite the clear difference in end-diastolic diameter.

\subsubsection{In vitro results}

At each of the three measurement positions (1), (2) and (3) (Fig. 6.2) in the distensible tube flow velocity distribution was assessed from ultrasound and LDA data. Fig. 6.4a and $6.4 \mathrm{c}$ depicts the flow velocity pattern at position (2) assessed by means of ultrasound and LDA, respectively. Only flow velocity distribution in half of the tube is shown because of symmetry and the better visual interpretation in relation to the velocity axis. The difference between the flow velocity distributions as assessed at the measurement positions (1), (2) and (3) with ultrasound and LDA are shown in Fig. 6.5. The root mean square (RMS) difference in flow velocity distribution as assessed with ultrasound and LDA at the measurement positions (1), (2) and (3) was $20 \mathrm{~mm} / \mathrm{s}$. This RMS difference normalized to a mean center stream flow velocity of $235 \mathrm{~mm} / \mathrm{s}$ was $8.6 \%$. 
The shear rate distribution at the three measurement positions was computed from the flow velocity distribution by means of:

$$
\gamma(\mathrm{r}, \mathrm{t})=\frac{\partial \mathrm{v}(\mathrm{r}, \mathrm{t})}{\partial \mathrm{r}}
$$

The shear rate distribution at position (2) is shown in Fig. 6.4. Fig. 6.4b gives the shear rate distribution derived from the ultrasound velocity distribution while Fig. $6.4 \mathrm{~d}$ provides the shear rate distribution derived from the LDA velocity distribution. The RMS difference in the derived shear rate distributions at the measurement positions (1), (2) and (3) was $35 \mathrm{~s}^{-1}$. This RMS difference normalized to a mean peak wall shear rate of $661 \mathrm{~s}^{-1}$ was $5.3 \%$.

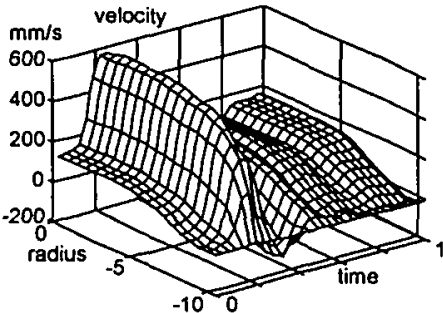

(a)

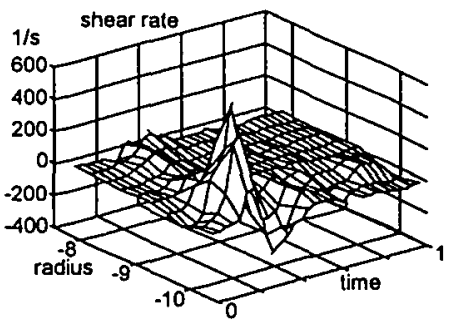

(b)

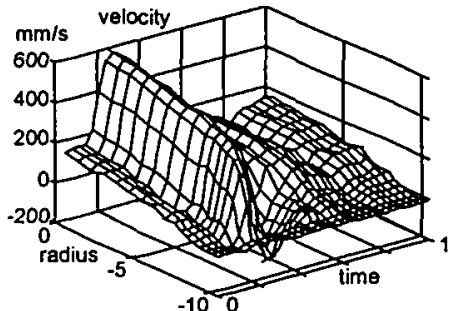

(c)

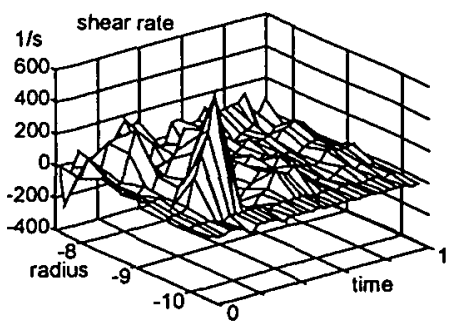

(d)

Figure 6.4: The velocity distribution at position (2) in the in vitro setup as assessed with ultrasound (a) and with LDA (c) together with the shear rate distribution as derived from the velocity distribution based on ultrasound (b) and LDA (d). 


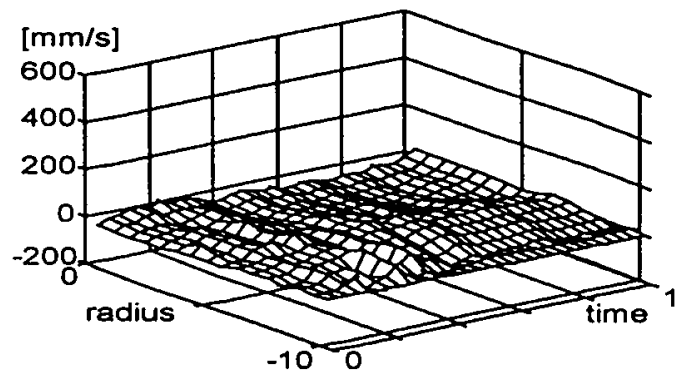

(1)

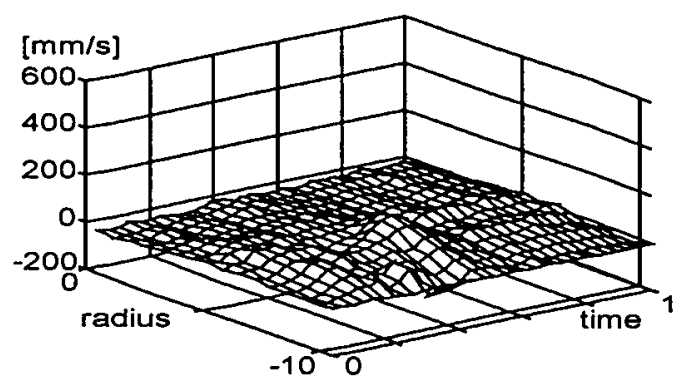

(2)

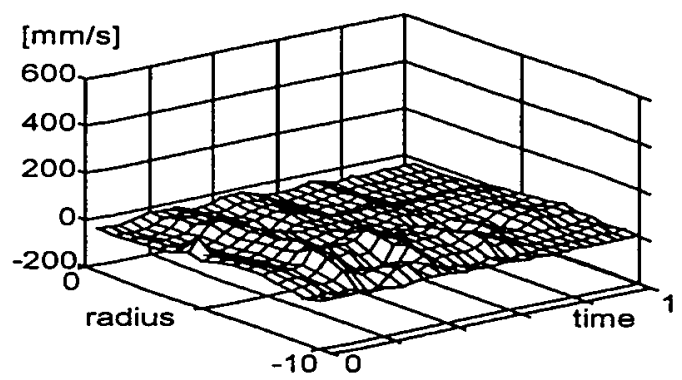

(3)

Figure 6.5: The difference in flow velocity distributions at the three measurement positions (1), (2) and (3) as assessed with ultrasound and with LDA. 
(A)

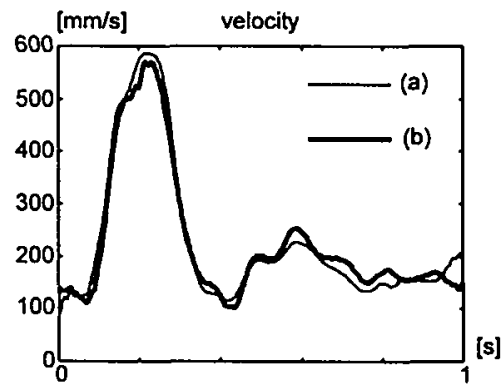

(1)

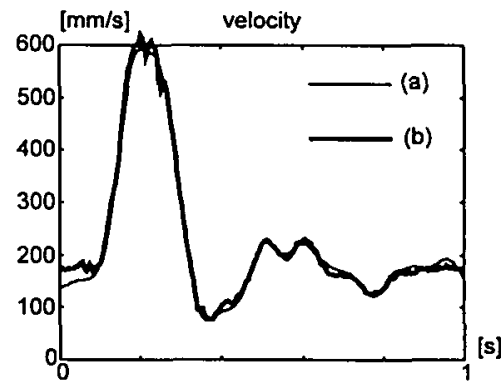

(2)

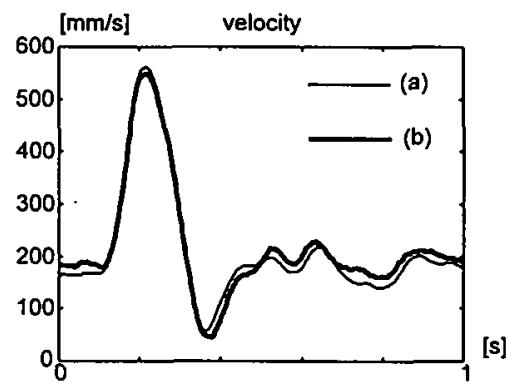

(3)
(B)

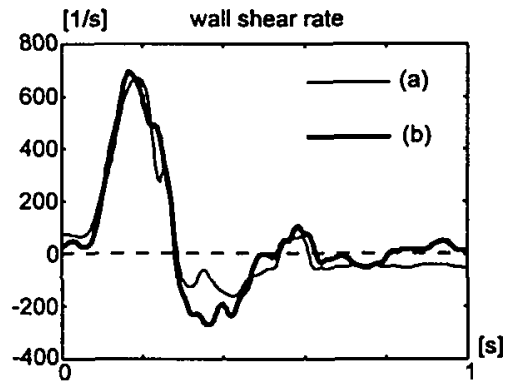

(1)

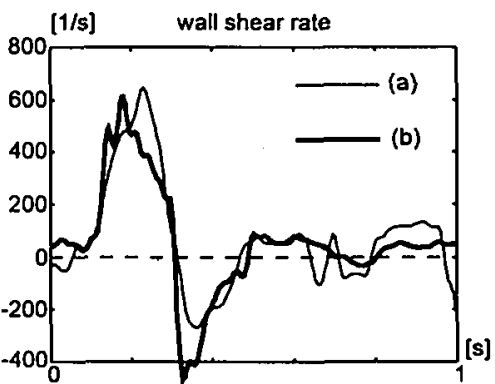

(2)

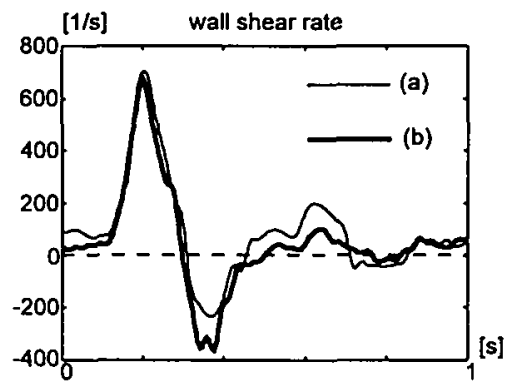

(3)

Figure 6.6: (A) The center stream flow velocity at the three measurement positions (1), (2) and (3) as assessed with ultrasound (a) and with LDA (b). (B) The wall shear rate waveforms at the three measurement positions (1), (2) and (3) as derived from the velocity distributions based on ultrasound (a) and on LDA (b) measurements.

Fig. 6.6A shows the center stream flow velocities at the three measurement positions (1), (2) and (3) as assessed with ultrasound and LDA. The RMS difference in center stream flow velocity as assessed with both methods at the positions (1), (2) and (3) was $9.6 \mathrm{~mm} / \mathrm{s}$. This RMS difference normalized to a mean center stream flow velocity of $235 \mathrm{~mm} / \mathrm{s}$ was $4.1 \%$. 
The wall shear rate waveform was estimated from the shear rate distribution (Fig. 6.4) within an region of $0.9 \mathrm{~mm}$ from the wall-lumen transition by means of

$$
\gamma(\mathrm{t})=\left.\frac{\partial \mathrm{v}(\mathrm{r}, \mathrm{t})}{\partial \mathrm{r}}\right|_{\text {MAX-0.9 }}
$$

Fig. 6.6B shows the wall shear rate estimations as obtained from the velocity distributions at the positions (1), (2) and (3) by means of expression (6.6). The RMS difference in wall shear rate waveforms at the measurement positions (1), (2) and (3) was $62 \mathrm{~s}^{-1}$. This RMS difference normalized to a mean peak wall shear rate of $661 \mathrm{~s}^{-1}$ was $9.4 \%$.

The wall shear rate in the in vitro setup was also estimated from a modelled flow velocity distribution (6.3) in combination with the diameter, as described by expression (6.4). The mean bluntness factor $n$ of the flow velocity distributions as assessed with ultrasound was 3.1 while for the flow velocity distributions as assessed with LDA it was 2.9. Fig. 6.7a gives the model based wall shear rate (6.4) using ultrasound data, while Fig.6.7b provides the corresponding results for LDA data. The differences in wall shear rate waveforms obtained with both methods were small because of the small differences in center stream flow velocity, diameter, and bluntness factor. However, the RMS difference between the direct approach of wall shear rate estimation and the model based approach was $171 \mathrm{~s}^{-1}$. This RMS difference normalized to a mean peak wall shear rate of $661 \mathrm{~s}^{-1}$ was $26 \%$.

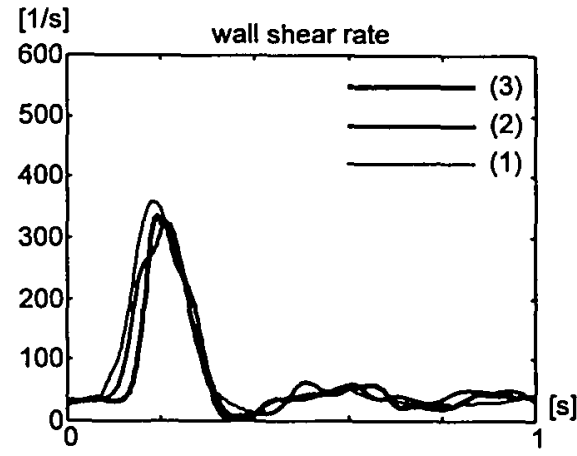

(A)

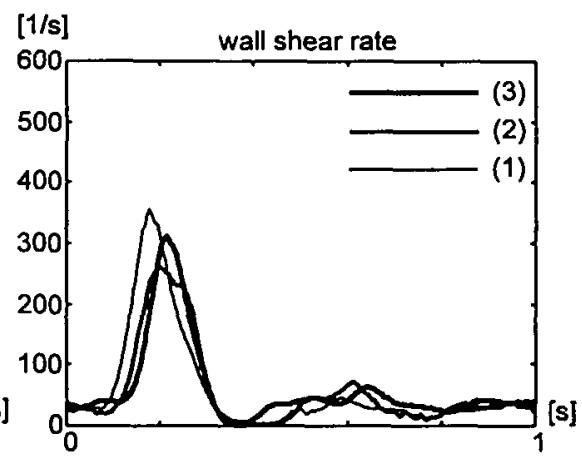

(B)

Figure 6.7: (A) The model based wall shear rate waveforms at positions (1), (2) and (3) as computed from ultrasound data. (B) The model based wall shear rate waveforms at positions (1), (2) and (3) as computed from LDA data. 


\subsection{Discussion}

Despite the widespread interest in (mean) wall shear rate and wall shear stress relatively little is known about the values of these mechanical entities in vivo (Simon et al. 1990, Gibson et al. 1993). The basic problem in the determination of wall shear rate in vivo, preferably non-invasive, concerns the measurement of the velocity gradient close to the wall with a minimum of interference. Only recently data appeared in literature on the assessment of instantaneous wall shear rate in the abdominal aorta of dogs (White et al. 1994), employing hot-film anemometry. However, the implantation of the transducers involves a far-reaching surgical procedure, making the method unsuitable for application in man.

Employing dedicated ultrasound techniques it is possible to assess in a non-invasive way the instantaneous velocity distribution over time. A high velocity and spatial resolution is achieved by capturing the received RF-signals over a limited time window (one cardiac cycle) and processing the signals off-line, paying specific attention to selective clutter suppression resulting in an extension of the estimation range to lower velocities (Chapter 4).

The major difference between the signal processing as used for the study in this Chapter and that in Chapter 5 is in the noise reduction procedure applied to the estimated twodimensional velocity distribution. In Chapter 5 a combination of a 3 by 3 median filter and a 3 by 3 smoothing window was employed while in this Chapter only the median filter was activated. A specific characteristic of a median filter is the retainment of level transitions (edges) which is of major importance for the estimation of the local shear rate. On the other hand a smoothing filter will tend to remove the edges and will reduce the velocity gradient, resulting in lower shear rate estimates. The additional smoothing filter modifies in an unacceptable way the estimated velocity distribution as function of depth. On the other hand the less extensive smoothing explains the larger standard deviation and the larger coefficients of variation of the estimate as compared with the in vivo results in Chapter 5 (Table 5.1).

The resolution perpendicular to the axis of the artery is governed by the system bandwidth in combination with the angle of observation and the local width of the ultrasound beam. Since an angle of $70^{\circ}$ degrees was employed the major restriction for the radial resolution originates from the duration of the emitted ultrasound burst (here 2 periods at $5 \mathrm{MHz}$ ), the bandwidth of the transducer and the smoothing procedures employed to reduce the variance of the velocity estimates. Matching the sample windows to the burst duration gives windows corresponding to $0.3 \mathrm{~mm}$ and spaced at $0.15 \mathrm{~mm}$ along the beam. Considering the angle of observation the interspacing converts to radial increments of $0.14 \mathrm{~mm}$. An observed wall shear rate of, for example, $1000 \mathrm{~s}^{-1}$ then corresponds to a peak velocity gradient between successive sample gates of $140 \mathrm{~mm} / \mathrm{s}$. This implies that starting from the wall at this shear rate a maximum velocity of $700 \mathrm{~mm} / \mathrm{s}$ (younger age group) can be reached within 5 sample gates or 0.75 
$\mathrm{mm}$, supporting the claim about radial resolution. Some subjects with a relatively high systolic center-line blood flow velocity indeed showed peak shear rates of up to $1400 \mathrm{~s}^{-1}$ and mean wall shear rates of up to $420 \mathrm{~s}^{-1}$ indicating that the system is capable to resolve high spatial velocity gradients.

Assuming a blood viscosity of $3.5 \mathrm{mPa} . \mathrm{s}$ a mean wall shear rate of $340 \mathrm{~s}^{-1}$ corresponds to a mean wall shear stress of $1.2 \mathrm{~Pa}$, which is somewhat lower than the shear stresses reported in the literature (Rodbard 1975, Kamiya and Togawa 1980, LaBarbera 1990). As demonstrated with computer models (Perktold et al. 1994) and in in vitro experiments (Duncan et al. 1990) the elastic behavior of the artery wall results in a reduced wall shear rate. This is not taken into account when wall shear rate is computed from volume flow and arterial diameter (6.4) based on a modelled flow velocity distribution (6.3). This effect is illustrated by the computed wall shear rates (Fig. 6.7) based on (6.4) with the bluntness factor estimated from the in vitro velocity distributions at the three measurement positions (Fig. 6.2). The computed values are substantially lower than obtained with the direct method of assessment (Fig. 6.6). Apparently the pulsatile nature of the flow velocity in an artery (tube) complicates wall shear rate estimation from the assumed shape of the velocity distribution. The other possibility is that the direct method overestimates wall shear rate in the diastolic phase of the cardiac (pump) cycle, which mainly determines the mean wall shear rate because of its relative duration. A time window of $10 \mathrm{~ms}$ limits the minimum width of the wall rejection filter to $100 \mathrm{~Hz}$ (Section 3.3.2), centered at the temporal mean frequency of the clutter signals, which is close to zero in the diastolic phase. A cut-off frequency of 50 $\mathrm{Hz}$ is equivalent to an absolute minimum flow velocity of $22 \mathrm{~mm} / \mathrm{s}$ or an absolute wall shear rate of $70 \mathrm{~s}^{-1}$ at the wall lumen boundary (transition from zero flow velocity to a valid velocity). However, all in vivo registrations made exhibited a minimum value at end-diastole higher than the critical one, indicating that the velocity gradient was derived from the slope rather than from the edge of the instantaneous velocity profile. The absolute wall shear rate of the in vitro registrations exhibited at a few instances in the diastolic phase of the pump cycle an absolute minimum value that was lower than the critical one. This may explain the sudden fluctuations in the ultrasound wall shear waveforms between a positive and a negative value in the diastolic phase of the pump cycle (Fig. 6.6). The wall shear rate registrations as obtained from the LDA measurements do not show the sudden fluctuations because there is no clutter filter in the signal processing which will introduce a minimum flow velocity. On the other hand, the flow velocity distributions as assessed with LDA are based on ensemble averaging of 32 repetitive measurements in time while the flow velocity distribution as assessed with ultrasound is based on a single recording.

The coefficient of variation of the estimated peak-to-peak distension ranged from 8 to $34 \%$ (mean CV $13 \%$ and $20 \%$ for the younger and older age group, respectively). This is considerably higher than the values reported earlier for the reproducibility of wall distensibility measurements in the carotid and femoral arteries (Kool et al. 1994). However, in the latter study a registration comprised at least 5 heart beats whereas in 
the present study each measurement is based on a single heart cycle, explaining for a factor of 2.2 between the standard deviations observed in both studies.

The angle of observation affects both the depth and velocity scaling. Since the shear rate is defined as the rate of change in velocity per change in radial position a minor change in observation angle may have large effects on the observed shear rate estimate. An error of only one degree in repositioning the ultrasound beam with respect to the artery axis induces an error of $4 \%$ or $6 \%$ in the shear rate estimate at an angle of $60^{\circ}$ or $70^{\circ}$ degrees, respectively. The rather steep angle of $70^{\circ}$ degrees had to be chosen because of the trade off between pulse repetition frequency, maximum velocity without aliasing, the anticipated duration of a cardiac cycle, and the memory size of the data acquisition unit. For the given size of the memory a higher pulse repetition frequency will shorten the registration time. However, a higher pulse repetition frequency will allow for a less steep angle of observation and a more reliable SNR estimation of the blood induced velocity signals because of the concomitant reduction in the fractional bandwidth of the temporal frequency distribution (Section 2.3.1).

The use of an additional emitter/receiver unit circumvents the practical limitations of commercially available echo systems but has several drawbacks. First the additional cable attachment promotes RF interference and noise sensitivity. The practical problems associated with the system configuration can only be eliminated if echo-systems support a sufficiently high pulse repetition frequency in echo M-mode (for peripheral applications exceeding $10 \mathrm{kHz}$ ) in combination with a sample clock that is phase locked to the trigger for emission. Systems of this kind are presently not yet (commercial) available. In the pilot study on the reproducibility of peak wall shear rate as described in Section 5.6 a prototype of a color flowmapper (developed at the department of Biophysics, University of Limburg) was used. This system supports a sufficiently high pulse repetition frequency in echo $\mathrm{M}$-mode $(8 \mathrm{kHz})$ in combination with a phase locked sample clock but it can not be used in a clinical environment.

A direct check on the quality of the assessed velocity distribution is possible by comparing the center stream peak velocities obtained with both the commercially echo scanner and the shear rate system. The echo scanner in pulsed Doppler mode permits the assessment of the peak value of the spectral envelope rather than the spectral mean. Because of system bandwidth considerations the estimated maximum will be 10 to $20 \%$ higher than the actual peak velocity. For that reason the cut-off point for acceptance of the systolic peak velocity of the shear rate system was set at $80 \%$ of the peak value of the spectral envelope. The rejection rate for the older age category was substantially higher than for the younger age group. This can be attributed to the way the common carotid artery was tethered to the surrounding tissue, fat content, and to the diminished respiration control of the older subjects.

In this Chapter the method for wall shear rate measurements was applied to the common carotid artery of adult persons and to flow in a distensible tube under non-stationary 
flow conditions resembling the flow pattern in the descending aorta. The internal diameter of the common carotid artery ranged from 6 to $7 \mathrm{~mm}$ while the center stream flow velocity, averaged over a cardiac cycle, was on the order of $300 \mathrm{~mm} / \mathrm{s}$. For arteries in the periphery the diameters will be smaller than $6 \mathrm{~mm}$ and the anticipated peak wall shear rate will be higher (Kamiya et al. 1984). This will put a higher demand on the resolution of the ultrasound system. For the present configuration, operating at $5 \mathrm{MHz}$ and an emission pulse duration of 2 to 3 periods, the application has to be restricted to the larger arteries.

\subsection{Conclusion}

Non-invasive ultrasound techniques can be employed to assess the wall shear rate waveform in the major peripheral arteries as carotid and femoral artery in humans. Our in vitro study shows that the mean difference in wall shear obtained with ultrasound and LDA is on the order of $9.4 \%$. This value indicates that ultrasound systems can adequately estimate the wall shear rate. Our in vivo repeatability study shows that wall shear rate can be assessed with a mean coefficient of variation of $13 \%$, a value similar in young (20-30y) and old (60-70y) age groups. Moreover, the in vivo study shows that the observed mean wall shear rate is the same $\left(340 \mathrm{~s}^{-1}\right)$ for young and old age category despite the differences between both groups in flow velocity characteristics (peak systolic and mean flow velocity) and in end-diastolic artery diameter. The anticipated autoregulatory interaction between vessel caliber and wall shear stress is corroborated by the observation that the velocity gradient at the wall is not affected by the diameter. Although the system provides satisfactory results its performance may be improved. This may specifically be achieved by improving the additional emitter/receiver unit, the pulse repetition frequency in M-mode, and the size of the memory to store temporary the acquired RF-matrix. 


\section{Chapter 7}

\section{A non-invasive method to estimate dimensionless arterial impedance by means of ultrasound}

7.1 Abstract 112

7.2 Introduction 112

7.3 Method of measurement. 114

7.3.1 Ultrasound arterial impedance assessment.....................................114

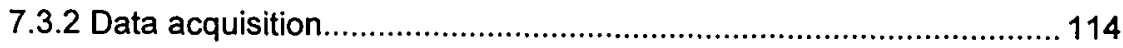

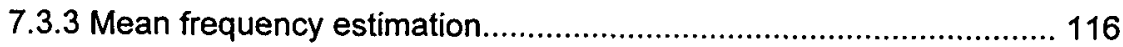

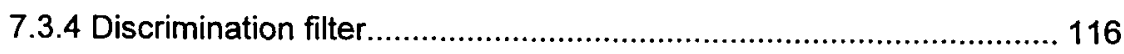

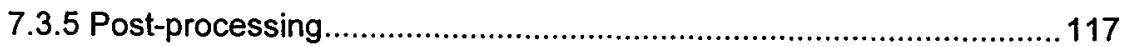

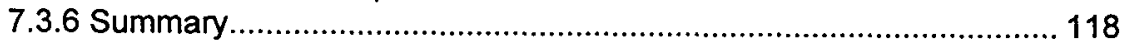

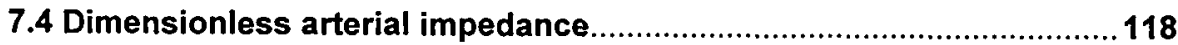

7.4.1 Relation volume flow and center-line flow velocity........................ 118

7.4.2 Relation local pressure and distension waveform............................ 120

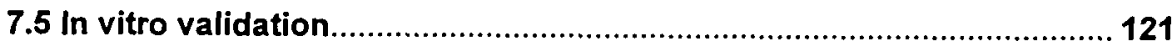

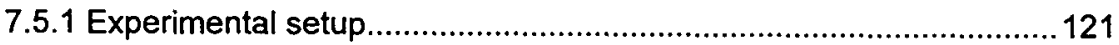

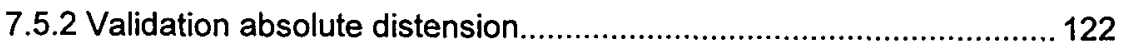

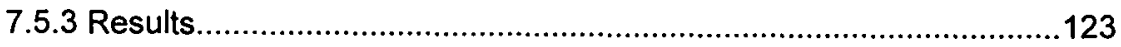

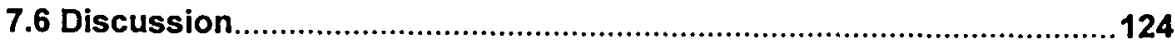

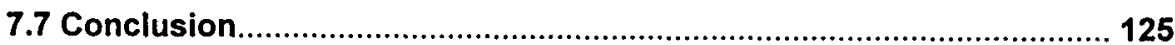

This chapter is based on an article, which has been accepted for publication in: Ultrasound in Medicine and Biology (January 1996). Peter J. Brands, A.P.G. Hoeks, M.C.M. Rutten, R.S. Reneman A non-invasive method to estimate arterial impedance by means of the assessment of the local diameter change and the local center-line blood flow velocity using ultrasound. 


\subsection{Abstract}

Vascular impedance is defined as the ratio between the frequency components of the local blood pressure waveform and those of the local blood volume flow waveform. Assessment of vascular impedance is, for example, important to study heart load and distal vascular beds vasomotricity. However, only few studies on vascular impedance have been performed in humans because pulsatile pressure and volume flow waveforms, simultaneously recorded at the same location, are difficult to obtain noninvasively. The non-invasive assessment of arterial impedance as described in this chapter is based on the replacement of the pressure waveform by the distension (change in diameter) waveform and the volume flow waveform by the center-line blood flow velocity waveform. Both waveforms can simultaneously and accurately be assessed by means of pulsed ultrasound. It will be shown that depending on the Womersley number the volume flow waveform may be replaced by the center-line blood flow velocity waveform for a given frequency range and that the pressure waveform may be replaced by the distension waveform for a wide frequency range. The validation of the proposed ultrasound method was performed through an in vitro study in a flow model with a distensible tube terminated with a hydraulic load (modified windkessel model). It is shown that in vitro the proposed method gives the same results as the local spectral pressure-flow relationship.

Key words: distension, pulsed ultrasound, RF cross-correlation, signal processing, arterial impedance.

\subsection{Introduction}

The subject of this chapter is to describe a non-invasive method to estimate arterial impedance using, RF-domain ultrasound signal processing.

Arterial impedance describes the spectral relationship between blood pressure and blood volume flow of an artery and characterizes the properties of the vascular bed downstream. The independence of arterial impedance of the input volume flow (Fig. 1.4) makes arterial impedance of particular value in studies on heart load, vascular circulation and distal vascular beds vasomotricity (O'Rourke and Taylor 1967, Westerhof and Noordergraaf 1970, Westerhof and Elzinga 1974, Milnor 1975, O'Rourke 1982, Powalowski 1989). The information about arterial impedance presently available is mainly based on invasive measurements in animals (Randall and Stacey 1956, Attinger et al. 1966, Gow and Taylor 1968, Farrar et al. 1978) because studies on arterial impedance in humans are hampered by the lack of reliable non-invasive techniques to record pressure waveforms locally. To overcome this limitation the local distension (change in diameter) waveform, will be used as a substitute for the local blood pressure waveform, while the local center-line blood flow velocity waveform is used as a substitute for the local blood volume flow waveform. Both waveforms can simultaneously and accurately be assessed by means of pulsed ultrasound. The ratio of 
the spectral components of the normalized distension waveform and the normalized center-line blood flow velocity waveform provides an estimate of dimensionless arterial impedance (ultrasound arterial impedance). Normalization with respect to peak-to-peak excursions within a cardiac cycle (Fig. 7.1) is used because then no assumptions have to be made for the conversion of the distension waveform in absolute units of pressure (mmHg) and for the conversion of the center-line blood flow velocity in absolute units of flow ( $1 / \mathrm{min}$ ) both as function of time (Powalowski 1989). The consequence of this approach is that the ultrasound arterial impedance obtained is dimensionless and that the shape of distension and center-line blood flow velocity waveforms are considered rather than the incidental magnitude of these waveforms.
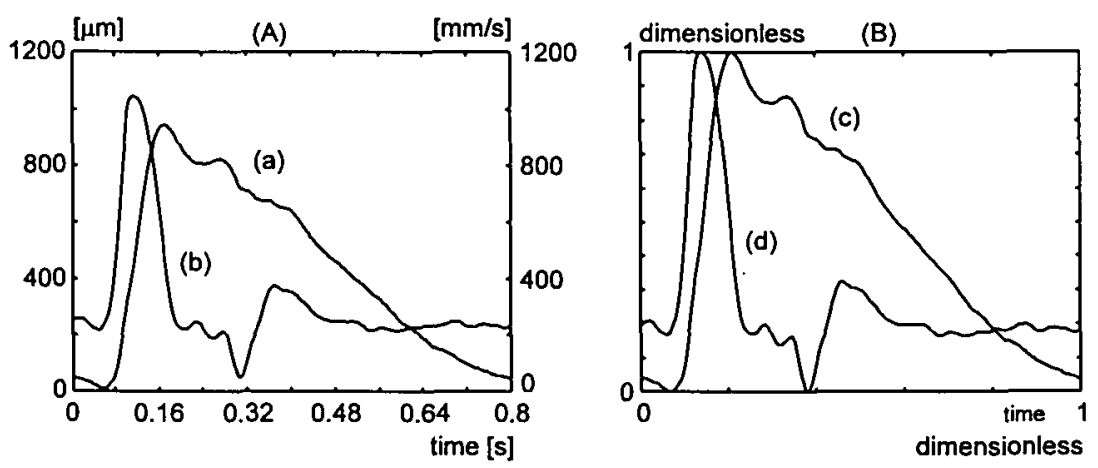

Figure 7.1: (A) The distension in $\mu \mathrm{m}$ (a) and center-line blood flow velocity in $\mathrm{mm} / \mathrm{s}$ (b) measured in the common carotid artery. (B) The normalized distension (c) and the normalized center-line blood flow velocity (d).

The ultrasound signal processing necessary for the assessment of the local distension waveform and the local center-line blood flow velocity waveform is performed in the RF-domain and consists of a mean frequency estimator based on an RF crosscorrelation model (CCM), preceded by a static discrimination filter. The ultrasound system to assess arterial impedance consists of an ultrasound echo system (operating at an emission frequency of $6.1 \mathrm{MHz}$ ), a data acquisition system to digitize and store the ultrasound RF-signals received during a few seconds ( 1 to 4 ) as an RF-matrix, and a computer system to estimate off-line the dimensionless arterial impedance.

The ultrasound method to assess arterial impedance is validated in both an in vivo and an in vitro study. The in vivo study concerns a pilot study regarding the reproducibility of magnitude and phase of the harmonics of the dimensionless ultrasound arterial impedance. This reproducibility is based on 8 measurements of the distension and center-line blood flow velocity waveforms at the same location in the common carotid artery in twelve healthy volunteers varying in age between 20 and 69 years (Chapter 8). The in vitro study concerns a comparison of the dimensionless ultrasound arterial impedance with the dimensionless spectral relationship between local pressure and local 
volume flow (reference arterial impedance). The waveforms are obtained in a distensible tube terminated with a hydraulic load (modified windkessel model) under non-stationary flow conditions in which the flow wave resembles the aortic volume flow waveform.

\subsection{Method of measurement}

\subsubsection{Ultrasound arterial impedance assessment}

Arterial impedance $Z$ is determined in the frequency domain from successive harmonics $h$ of pressure and volume flow waveforms over a cardiac cycle as:

$$
Z\left(h . f_{0}\right)=\frac{P\left(h . f_{0}\right)}{Q\left(h . f_{0}\right)}=A\left(h . f_{0}\right) \cdot \exp \left(\phi\left(h . f_{0}\right)\right) \quad h=0,1,2 \ldots . H
$$

where $f_{0}$ is the fundamental frequency of a given heart beat, $P\left(h . f_{0}\right)$ the harmonics of the blood pressure waveform, $Q\left(h . f_{n}\right)$ the harmonics of the blood volume flow waveform, and $A\left(h . f_{0}\right)$ the magnitude and $\phi\left(h . f_{0}\right)$ the phase of the arterial impedance, respectively. If $h=0$, the ratio of mean blood pressure and mean blood volume flow is computed, giving the peripheral resistance.

The method to non-invasively determine arterial impedance as presented in this chapter is based on the assessment of the ratio of the harmonics of the normalized change in diameter waveform during the cardiac cycle $\Delta d(t)$ and the harmonics of the normalized center-line mean blood flow velocity waveform during the cardiac cycle $\bar{v}(t)$ as:

$$
Z_{D V}\left(h \cdot f_{0}\right)=\frac{D_{n}\left(h \cdot f_{0}\right)}{V_{n}\left(h \cdot f_{0}\right)}=A_{n}\left(h \cdot f_{0}\right) \cdot \exp \left(\phi\left(h \cdot f_{0}\right)\right) \quad h=0,1,2 \ldots . H
$$

where $Z_{D V}$ is the dimensionless ultrasound arterial impedance, $A_{n}\left(h . f_{D}\right)$ its magnitude and $\phi\left(h f_{0}\right)$ its phase. Replacement of the volume flow waveform by the center-line flow velocity waveform and the pressure waveform by the distension waveform assumes a linear relationship between the related parameters. As will be demonstrated this linear relationship is an acceptable assumption for elastic arteries like the common carotid artery.

\subsubsection{Data acquisition}

The acquired RF-signals are stored as an RF-matrix organized in time and as three regions in depth: 1) $64 \mathrm{RF}$ sample points of the anterior vessel wall reflection, 2) $32 \mathrm{RF}$ sample points in the center of the lumen of the blood vessel and 3) $64 \mathrm{RF}$ sample points of the posterior vessel wall reflection (Fig. 7.2). 


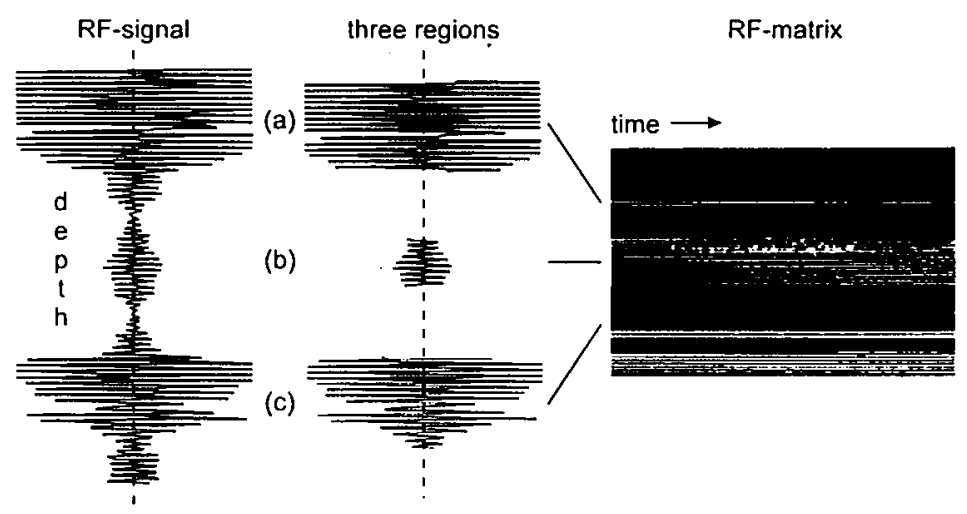

Figure 7.2: Data storage of the acquired RF-signals in an RF-matrix as function of time from three regions in depth: (a) anterior vessel wall, (b) center of the lumen and (c) posterior vessel wall.

The reason for using these three specified regions rather than a continuous depth range is to reduce the amount of RF-data to be stored. Moreover, only the distension and center-line blood flow velocity waveforms are relevant to obtain an estimate of the dimensionless ultrasound arterial impedance. The echo system is operated in M-mode to retain a high axial resolution. This does not conflict with velocity estimation because of the RF-processing employed. The temporary storage of the acquired RF-signals has the advantage that the RF-matrix (Fig. 7.2) can be retained on a backup medium (DAT or WORM-CD) for further off-line analysis. The acquisition system samples and stores the RF-signals in real-time in the internal memory of the computer system. It has a synchronous (with the emission trigger of the attached echo system) sample frequency of four times the emission frequency $(24.4 \mathrm{MHz}$ ), a dynamic range of $72 \mathrm{~dB}$ (12 bit) and is connected to the computer system through a VESA-local bus. The latter is required to achieve a high data throughput rate (up to $10 \mathrm{Mbyte} / \mathrm{sec}$ ) to the internal memory of the computer system. For the dimensionless arterial impedance measurements the acquisition memory is organized as 32,768 temporal RF-signals of 160 spatial sample points each (64 anterior, 64 posterior and 32 lumen center sample points), requiring an acquisition memory size of 10 Mbyte. At a PRF of $8 \mathrm{kHz}, 32,768$ temporal RF-signals are equivalent to $4 \mathrm{sec}$. Thus, the RF-matrix consists of $4 \mathrm{sec}$ of temporal and $5 \mathrm{~mm}$ of spatial ultrasound information $(2 \mathrm{~mm}$ from the anterior and posterior vessel wall together with $1 \mathrm{~mm}$ from the center of the lumen).

In order to select the relevant RF-samples and to restrict user interference an automatic method rather than a manual positioning procedure is used to identify the wall-lumen interface (Hoeks et al. 1995). The identification of the lumen of a blood vessel by the user activates data acquisition which starts synchronously with a trigger derived from the R-wave of an ECG-signal. The identification of the lumen, based on the envelope of the RF-signals, is necessary for the automatic detection of the wall-lumen interface 
(vessel walls) synchronously with the start of data acquisition. The method is based on the generation of dynamically adjusting threshold levels, independent for both vessel walls, using attack-sustain low-pass filters with a response time on the order of the response time of the depth-dependent gain control (approximately $10 \mu \mathrm{s}$ ). The accuracy and reproducibility of the automatic detection has been verified. The verification results show that the proposed method is consistent with a standard deviation of half the resolution of the attached echo-system.

\subsubsection{Mean frequency estimation}

The distension and center-line blood flow velocity waveforms are assessed with the RFdomain cross-correlation (CCM) estimator (Section 2.3.8) because of its high quality for wide bandwidth RF-signals. The estimated dimensionless velocity $\hat{\varphi}_{s}$ of the scattered signal and the dimensionless velocity $\hat{\varphi}_{r}$ of the reflected signal are related to the centerline mean blood flow velocity and the velocity of vessel wall displacement by means of expression (2.41). Vessel wall motion is obtained from the integrated velocity of vessel wall displacement on a beat to beat base. The distension waveform as function of time is obtained from the difference in motion between the anterior and the posterior vessel wall. The spatial estimation window length of the CCM estimator was selected according to the length of the emitted ultrasound burst ( 2 wavelengths), while the temporal estimation window length was selected according to the temporal behavior of the blood flow velocity. For a $6.1 \mathrm{MHz}$ pulsed echo system emitting 2 wavelengths the spatial estimation window was set at 8 spatial sample points, i.e., $250 \mu \mathrm{m}$ at a sample frequency of $24.4 \mathrm{MHz}$. A temporal estimation window of $10 \mathrm{msec}$ ( 80 temporal sample points at a PRF of $8 \mathrm{kHz}$ ) ensures a low standard deviation of the velocity estimate, while it does not compromise the temporal behavior of the observed velocities.

\subsubsection{Discrimination filter}

Discrimination between the reflected signals and the scattered signals (Chapter 3 ) in the RF-matrix acquired (Fig. 7.2) is obtained by means of a static discrimination filter (Fig. 3.8). The characteristics of such a filter are based on general assumptions of the frequency characteristics of the reverberations and the reflected signal, i.e., a low frequency, a narrow bandwidth and a high amplitude. The low-pass filtration to remove noise and scattered signals from the RF-signals received $\left(z_{r}\left(k_{0}, i\right)\right.$ Section 2.3.1) is obtained with a second order IIR low-pass filter (LPF). The cut-off frequency of the LPF is based on the assumed maximum velocity of vessel wall motion $(10 \mathrm{~mm} / \mathrm{s})$ and set at $40 \mathrm{~Hz}$ (Section 3.3.2) (Fig. 7.3). The high-pass filtration to remove the reflected signals is obtained with a second order IIR high-pass filter (HPF). The cut-off frequency of the HPF is set at $100 \mathrm{~Hz}$ in accordance with the temporal length of the estimation window (10 msec) (Fig. 7.3). The difference in amplitude between the reverberations in the center of the lumen and the scattered signal is on the order of $20 \mathrm{~dB}$. A suppression of $20 \mathrm{~dB}$ for a second order HPF (roll-off of $12 \mathrm{~dB} /$ octave) with a cut-off frequency of $100 \mathrm{~Hz}$ is achieved at frequencies lower than $32 \mathrm{~Hz}$. 


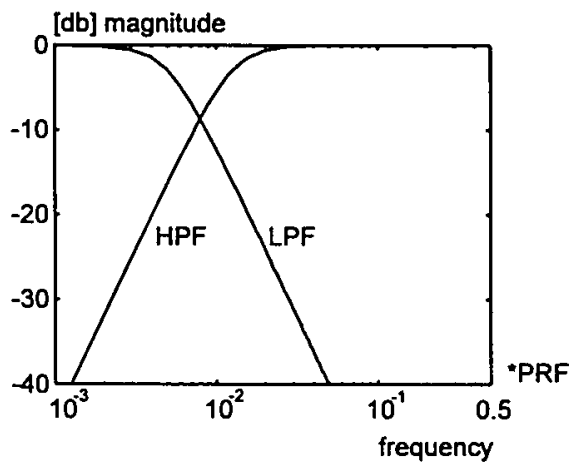

Figure 7.3: The transferfunction of the static discrimination filter used to estimate distension and center-line blood flow velocity to compute arterial impedance.

\subsubsection{Post-processing}

Post-processing of vessel wall motion involves a moving average filter with a 5 sample point line kernel acting on half overlapping sample windows spaced at $5 \mathrm{msec}$, giving a response time of $25 \mathrm{msec}(40 \mathrm{~Hz})$. The post-processing of the dimensionless velocity is carried out by means of a signal to noise ratio (SNR) decision rule, a median filter and a moving average filter. To reduce the variation of the estimated $\mathrm{SNR}_{\mathrm{s}}$ of the scattered signal, this $S \hat{N} R_{s}$ (4.4; Section 4.3) is subjected to a moving average filter with a 5 sample point line kernel ( $25 \mathrm{msec}$ response). If the smoothed $S \hat{N} R_{s}$ is less than $-3 \mathrm{~dB}$ $\left(S \hat{N} R_{s}<0.5\right)$ it may be expected that the estimation window does not contain a scattered signal and the corresponding estimate of the dimensionless velocity $\hat{\varphi}_{s}$ is set at zero.

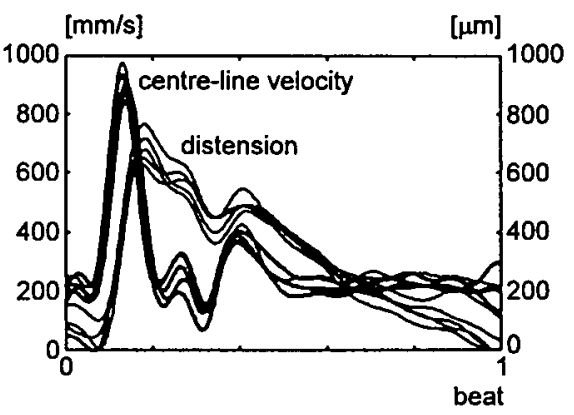

Figure 7.4: Estimated distension and center-line blood flow velocity waveforms over four heart beats measured in the common carotid artery.

To remove possible false positive and false negative decisions, inducing spot noise, $\hat{\varphi}_{s}$ is subjected to a median filter with a 5 sample point line kernel. Furthermore, $\hat{\varphi}_{s}$ is subjected to an averaging filter, with a 5 sample point line kernel, to remove the 
quantisation noise introduced by the median filter. Finally $\hat{\varphi}_{s}$ is converted to an index of blood flow velocity (Fig. 7.4).

\subsubsection{Summary}

The entire ultrasound signal processing procedure to estimate simultaneously distension and center-line blood flow velocity waveform consists of:

1) Identification of the vessel lumen boundary to distinguish three regions in depth and to store these signals in an RF-matrix as function of time (Fig. 7.2),

2) Discrimination with a static discrimination filter,

3) Estimation of the dimensionless velocities $\hat{\varphi}_{r}$ and $\hat{\varphi}_{s}$ of the reflected and the scattered signal, respectively, together with the SNR of the scattered signal,

4) Post-processing of the dimensionless velocity $\hat{\varphi}_{s}$ of the scattered signal and of the integrated dimensionless velocity $\hat{\varphi}_{r}$ (vessel wall motion) and

5) Conversion of $\hat{\varphi}_{s}$ and vessel wall motion to blood flow velocity and distension, respectively.

To reduce the computational load the processing steps two and three are performed in a temporal recursive way.

\subsection{Dimensionless arterial impedance}

From the equation of motion of a Newtonian fluid through a linearly elastic and isotropic tube the center-line blood flow velocity can be derived. The latter shows that for the frequency range between two and five $\mathrm{Hz}$ center-line flow velocity is almost linear with volume flow. From the force equilibrium of a tube it follows that distension is linear with pressure and that this linearity is not frequency dependent.

\subsubsection{Relation volume flow and center-line flow velocity}

From the Navier-Stokes equations coupled with the equilibrium equations of the tube the center-line blood flow velocity may be derived. This theory is applicable when harmonic solutions for pressure and volume flow are considered only and if the flow velocity is low in comparison with the pulse wave velocity (Womersley 1957). The Navier-Stokes equations will under these assumptions reduce to:

$$
\rho \cdot \frac{\partial v_{x}}{\partial t}=-\frac{\partial p}{\partial x}+\eta \frac{\partial}{\partial r}\left(\frac{\partial v_{x}}{\partial r}\right)
$$

where $\eta[\mathrm{Pa} . \mathrm{s}]$ is the dynamic viscosity, $\rho$ the specific mass of the fluid, $v_{x}$ the axial velocity component at the radial position $r$ and $\partial \mathrm{p} / \partial \mathrm{x}$ the axial pressure gradient. An analytical solution for the harmonics $h$ of the axial velocity profile $v_{x}(h, r, t)$ can be derived as: 


$$
v_{x}(h, r, t)=R E\left(\frac{R^{2}}{j \cdot \eta \cdot \alpha_{h}^{2}}\left[1-\frac{J_{0}\left(\alpha_{h} \cdot j^{3 / 2} \cdot r / R\right)}{J_{0}\left(\alpha_{h} \cdot j^{3 / 2}\right)}\right] \frac{\partial p(h, t)}{\partial x}\right) \quad h \geq 1
$$

where $\partial \mathrm{p}(\mathrm{h}, \mathrm{t}) / \partial \mathrm{x}$ is the $h^{\text {th }}$ harmonic of the axial pressure gradient, $R$ the mean radius of the tube, $J_{0}$ the Bessel function of the first kind of order zero, $\omega_{0}$ the angular frequency of a heart beat, $R E$ the real part operator and $\alpha_{h}$ the Womersley number defined as:

$$
\alpha_{\mathrm{h}}=\mathrm{R} \cdot \sqrt{\mathrm{h} \cdot \omega_{0} \cdot \frac{\rho}{\eta}}
$$

The Womersley number is a dimensionless measure for the ratio between instationary forces and viscosity forces. This number is for the first harmonic (heart rate) for the common carotid artery in the order of 5, for the aorta in the order of 15 and for the brachial artery in the order of 3.5. Integration of equation (7.4) over the vessel radius yields the relation between the harmonics of the local volume flow $Q(h, t)$ and the harmonics of the axial center-line flow velocity $v_{x}(h, r, t)_{r=0}$ as:

$$
v_{x}(h, r, t)_{r=0}=R E\left(\frac{1}{1-F_{10}\left(\alpha_{h}\right)}\right) \frac{Q(h, t)}{\pi \cdot R^{2}}
$$

with the Womersley function $F_{10}\left(\alpha_{h}\right)$ defined as:

$$
F_{10}\left(\alpha_{h}\right)=\frac{2 \cdot J_{1}\left(j^{3 / 2} \cdot \alpha_{h}\right)}{j^{3 / 2} \cdot \alpha_{h} \cdot J_{0}\left(j^{3 / 2} \cdot \alpha_{h}\right)}
$$

where $J$, is the Bessel function of the first kind order one.
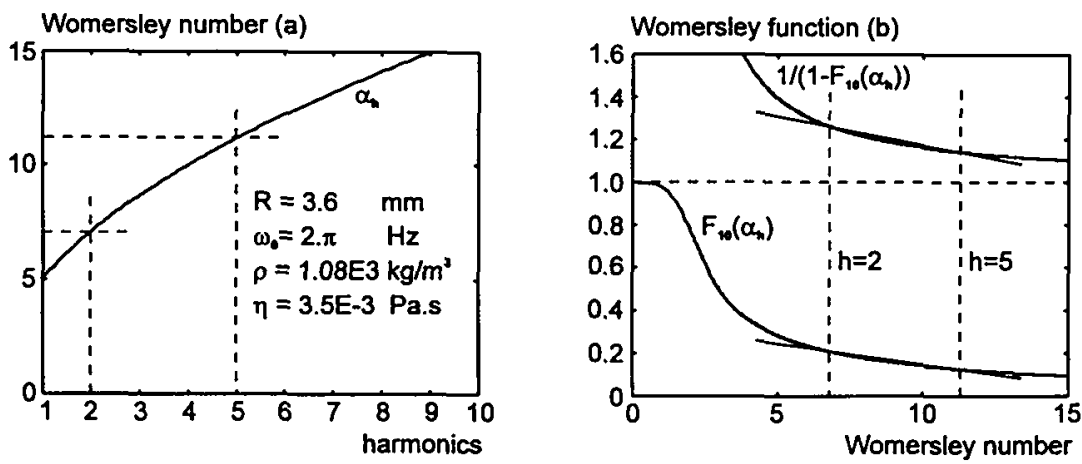

Figure 7.5: (a) The Womersley number $\alpha_{h}$ in the common carotid artery $\left(\alpha_{l}=5\right)$ and (b) the real part of the function $1 /\left(I-F_{10}\left(\alpha_{h}\right)\right)$ and the Womersley function $F_{10}\left(\alpha_{h}\right)$.

From the volume flow center-line flow velocity relationship (7.6) it follows that, if $1 /\left(1-F_{10}\left(\alpha_{h}\right)\right)$ may be linearized for a given range of the Womersley number $\alpha_{h}$ the 
center-line blood flow velocity is almost linearly dependent on the flow $Q$ in that frequency range. In Fig. 7.5 it is shown that for a Womersley number $\alpha_{1}$ of the order of five (realistic value for the carotid artery see Fig. 7.5a), linearization of the function $1 /\left(I-F_{10}\left(\alpha_{h}\right)\right)$ is possible. The signal power of the distension and center-line blood flow velocity is concentrated in the lower harmonics (Fig. 7.6). Therefore the linearization of the function $1 /\left(1-F_{l 0}\left(\alpha_{h}\right)\right)$ is applied to the part of the spectrum between the second and the fifth harmonic.

\subsubsection{Relation local pressure and distension waveform}

The distension waveform of the vessel wall is obtained from the integrated velocities of the anterior and posterior wall displacements. From the equilibrium equation of a tube with radius $R$, wall thickness $h$ and pressurized with pressure $p$ the pressure-distension relation can be derived. This equation is based on the force equilibrium between pressure times radius and wall thickness times circumferential stress $\sigma_{\phi \varphi}$ as:

$$
h \sigma_{\varphi \varphi}=p \cdot R
$$

The circumferential stress for a linearly (visco)elastic thin-walled tube is defined as:

$$
\sigma_{\varphi \phi}=\frac{E(\omega) \varepsilon_{\varphi \phi}}{1-v^{2}}
$$

where $\varepsilon_{\varphi \varphi}$ is the strain and $E$ and $v$ the (complex) Young's modules and the Poisson ratio of the material, respectively. The strain in the circumferential direction is defined as:

$$
\varepsilon_{\phi \varphi}=\frac{u_{r}}{\bar{R}}
$$

with $u_{r}$ the radial wall displacement and $\bar{R}$ the mean vessel radius. The pressuredistension relation then reads:

$$
u_{r}=\frac{\bar{R}^{2}\left(1-v^{2}\right)}{h E(\omega)} p=g(\omega) \cdot p
$$

The pressure-distension relation is a function of frequency $g(\omega)$ due to viscoelastic effects in the vessel wall. However, when the imaginary part of the Young's modules $E$ of the vessel material is small compared to the real part, the radial wall displacement is linear with pressure and not very frequency dependent. For blood vessels, this is indeed the case (Westerhof and Noordergraaf 1970, Milnor 1989). 

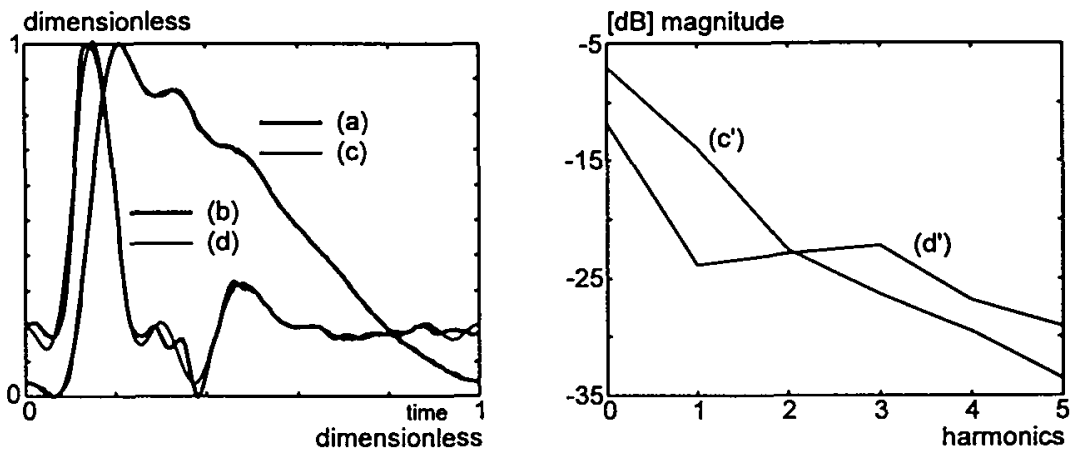

Figure 7.6: The normalized distension (a) and center-line blood flow velocity (b) as measured in the common carotid artery together with six harmonics of distension (c) and center-line blood flow velocity (d) in time and frequency domain (c') and (d').

\subsection{In vitro validation}

The validation of the method proposed to estimate dimensionless arterial impedance using pulsed ultrasound was performed through an in vitro study in a flow model with a distensible tube and a volume flow pulse with a Womersley number of $\alpha_{1}=15$. The in vitro experiments were performed at the department of Mechanical Engineering (WFW) of the Eindhoven University of Technology.

\subsubsection{Experimental setup}

The experimental setup is described in detail in Section 6.3.2.

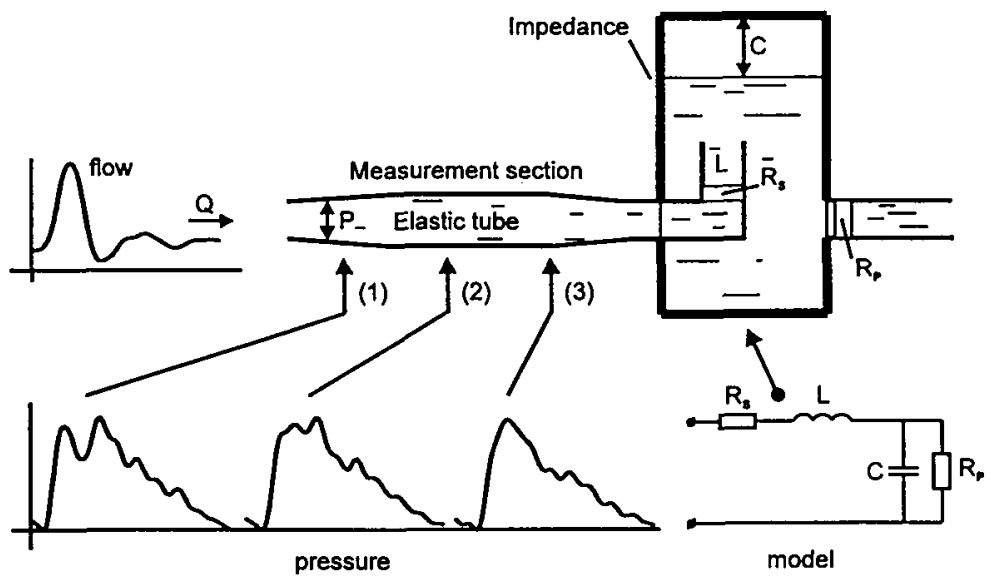

Figure 7.7: Schematic diagram of the in vitro setup composed of a distensible tube terminated with a hydraulic load. 
Distension of the tube and center-line flow velocity inside the tube were measured at three positions (Fig. 7.7, 1=106 mm, 2=231 mm and $3=356 \mathrm{~mm}$ downstream from the entrance of the tube) with pulsed ultrasound as described in Section 7.3. The volume flows proximal and distal to the tube were measured with electromagnetic flow probes (Skalar). The pressure in the tube was measured by means of a catheter-tip pressure transducer (Millar) at the three given positions simultaneously with distension. The local volume flow waveforms in the tube at the three positions were determined by integration of the velocity profiles measured with laser-Doppler anemometry.

\subsubsection{Validation absolute distension}

To validate the measurement of distension absolute distension is obtained from the static pressure-diameter relation at position two in the distensible tube and compared with the ultrasound distension measurement (Fig. 7.7). Pressure is increased stepwise by increasing the volume flow. At each pressure level the inner diameter of the tube was measured with laser light.
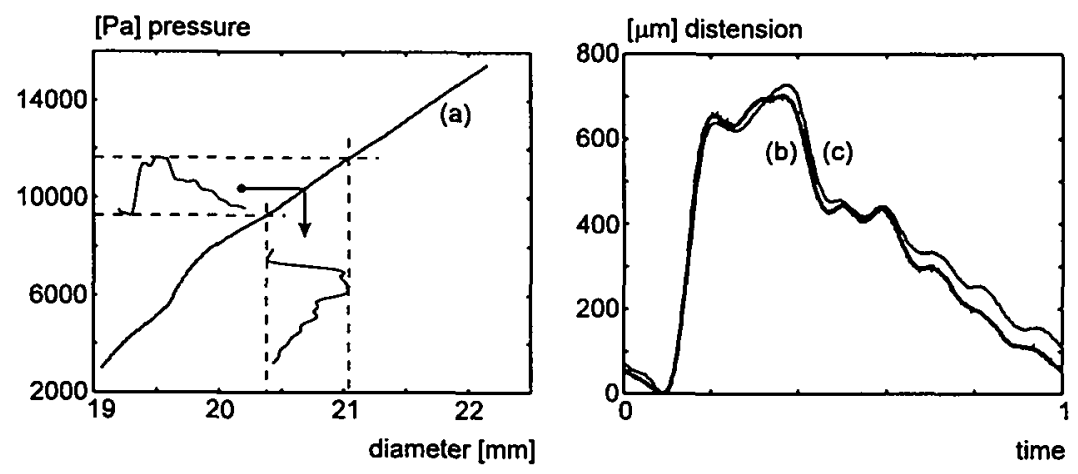

Figure 7.8: The absolute distension (b) as obtained by means of the static pressure-diameter relation (a) and the distension as assessed with ultrasound (c).

In Fig. 7.8 the mean values of ten measurements of absolute and assessed distension are shown. The coefficient of variation of the ten absolute distension curves as obtained by means of the static pressure-diameter relation was $5.2 \%$. The coefficient of variation of the ten measurements of pressure was $3.5 \%$. The normalized root mean square (RMS) difference between absolute distension and the distension as assessed by means of ultrasound (echo system $\mathrm{Q}=2$ ) was $1.6 \%$. This $1.6 \%$ is much lower than the coefficient of variation of the absolute distension because the variation in pressure contributes to pressure and absolute distension to the same extent. The time dependent bias (Fig. 7.8 right) originates from the CCM estimator and was $6.6 \%$ at the end of a pump cycle ( 1 $\mathrm{sec}$ ). Although the bias of the CCM estimator, due to the bandwidth assumption in the derivation of this estimator (Section 2.3.8) (Hoeks et al. 1993), is very low, it still 
contains a perceptible bias because of the integration of the velocity of vessel wall displacement. The value of this bias depends on the velocity and the quality factor of the ultrasound probe. A low bias is obtained for a high quality factor $(\mathrm{Q}>2)$, i.e., a low resolution.

For the validation of velocity assessment using ultrasound see Section 6.4.2.

\subsubsection{Results}

On each of the three measurement positions in the distensible tube ten measurements of the dimensionless reference arterial impedance and ten measurements of the dimensionless ultrasound arterial impedance were obtained. The ten repetitive measurements enabled estimation of the standard deviation. In Fig. 7.9 the results of a comparison of both methods are shown. Because of the relatively high Womersley number used $\left(\alpha_{1}=15\right.$, a realistic value for the aorta), above the first harmonic $(h>0)$ the reference arterial impedance is almost linear with the ultrasound arterial impedance.
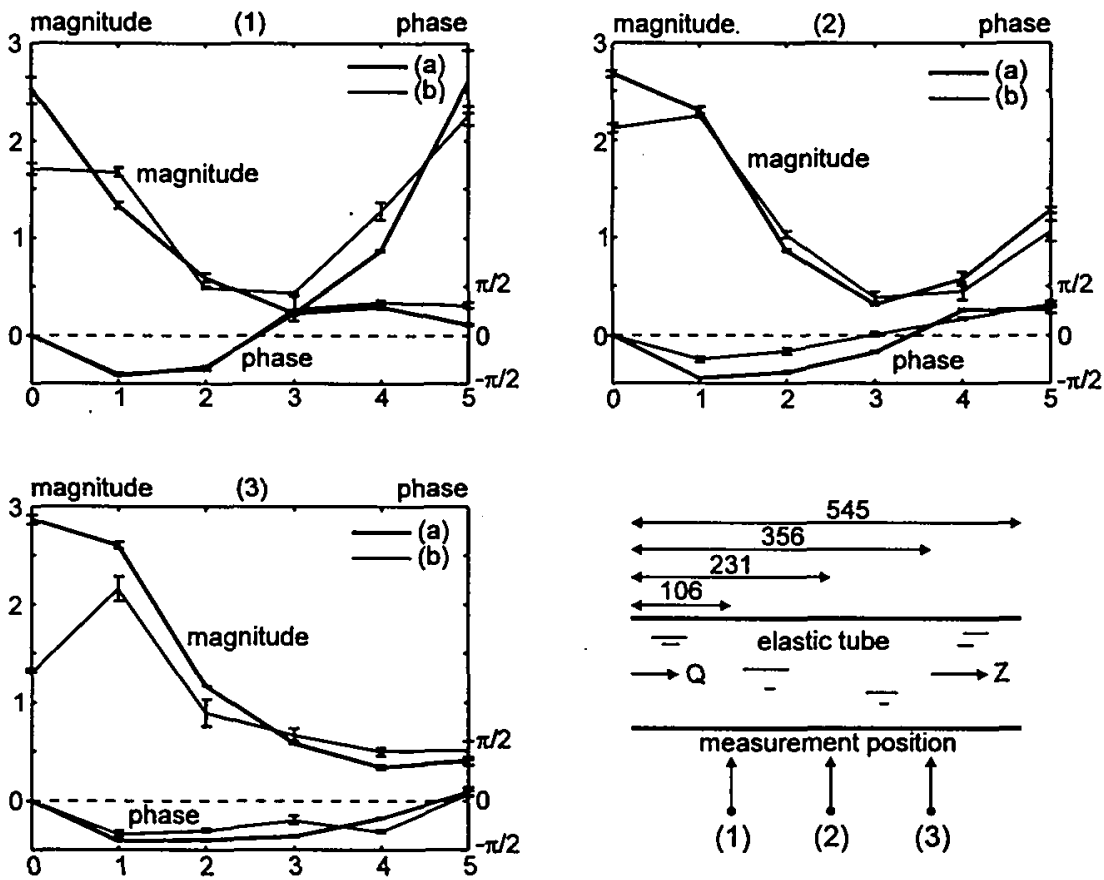

(1) (2) (3)

Figure 7.9: The magnitude and phase of the first six harmonics of the dimensionless reference arterial impedance (a) together with the dimensionless ultrasound arterial impedance (b) measured at three positions downstream the entrance of the tube (1), (2) and (3). 
The pressure and volume flow recordings are based on ensemble averaging of 32 repetitive measurements in time while distension and center-line flow velocity are based on a single recording. The normalized RMS difference in magnitude between the dimensionless reference arterial impedance and the dimensionless ultrasound arterial impedance at the three positions, for the second to fifth harmonic, was $11 \%$ (absolute \pm 0.14 ). The normalized RMS difference in phase at the three positions was $18 \%$ (absolute \pm 0.016 ). The reproducibility (coefficient of variation over the ten independent measurements) of magnitude and phase of the second to the fifth harmonics of the dimensionless ultrasound arterial impedance was $4 \%$. The reproducibility of the reference arterial impedance was $2.5 \%$.

\subsection{Discussion}

Despite the importance of the assessment of arterial impedance for the detection of stenoses and occlusions and to investigate the load for the left ventricle relatively few studies on arterial impedance in humans have been performed. The basic problem in the determination of arterial impedance in vivo pertains to the simultaneous recording at the same location of the volume flow and the pressure waveforms. As an alternative, Powalowski (Powalowski 1989) developed a non-invasive method to estimate arterial impedance based on simultaneous recording of distension and volume flow velocity by means of pulsed ultrasound and continuous wave ultrasound. In the method of Powalowski the conversion from the distension waveform to the pressure waveform is based on the assumption that there is a general parametric logarithmic relation between the cross-sectional area change of the common carotid artery and blood pressure measured in the brachial artery.

The method described in this chapter to obtain an estimate of the dimensionless arterial impedance in the common carotid artery is based on normalization. This normalization has the advantage that it is not necessary to calibrate the distension waveform using a conversion with the systolic and diastolic pressure measured in the brachial artery. However, the use of normalized distension and normalized center-line blood flow velocity makes it necessary to assume a linear relationship between distension and pressure and between center-line blood flow velocity and volume flow. Moreover, due to linearization and normalization it is impossible to estimate peripheral resistance $(h=0)$.

From the Womersley theory it follows that the linearity between center-line blood flow velocity and volume flow depends on heart rate, radius of the vessel and viscosity of blood. The relation between these variables and the harmonics of heart rate is given by the dimensionless Womersley number (7.5). Theoretically it follows that for a high Womersley number $\left(\alpha_{1}>5\right.$, a realistic value for the common carotid artery see Fig. $7.5 \mathrm{a}$ ), a pulse wave length substantially greater than the radius of the artery, a radial velocity at the wall smaller than the center-line velocity and a given range of harmonics (two to five), center-line blood flow velocity is linear with volume flow. This 
conclusion is supported by the results obtained in the in vitro experiments (Fig. 7.9). It is obvious that if the distension and center-line blood flow velocity waveforms are assessed in vessels affected by atheroma the assumed linear relationship between local distension and local blood pressure becomes invalid.

Ultrasound RF-domain signal processing allows to assess non-invasively, simultaneously and accurately the distension and center-line blood flow velocity waveforms. The ultrasound recordings are taken at an angle of $65^{\circ}$ with the vessel axis. The angle has no effect on the dimensionless arterial impedance because of the normalization of distension and center-line blood flow velocity. However, the angle

should be in the range between $60^{\circ}$ and $70^{\circ}$ to obtain a reliable velocity recording.

The discrimination between the scattered signal and the reverberations in the center of the lumen is obtained by means of a static high-pass filter. The cut-off frequency of this filter is set at $100 \mathrm{~Hz}$ according to the length of the temporal estimation window (10 $\mathrm{msec}$ ). Instead of a higher order IIR filter, a second order IIR filter is used because this will give an optimum between the effect on the bias of the estimate and the removal of the clutter (Tysoe and Evans 1995).

The accuracy of the ultrasound arterial impedance depends first of all on the accuracy of the distension and center-line blood flow velocity assessment, secondly on the linearity between distension and pressure and, finally, on the linearity between volume flow and center-line blood flow velocity for a given range of harmonics. The distension assessment is validated by means of the static relation between pressure and diameter in an elastic tube. The RMS difference between absolute distension and the distension as assessed with ultrasound was $1.6 \%$. This low value confirms the high quality of distension assessment by means of ultrasound. On the other hand the bias (over one heart cycle) between the absolute distension and the distension as assessed with ultrasound was $6.6 \%$ which is quite a high value. Although the bias in the estimate of the vessel wall velocity is very low, integration introduces a biased estimate of distension. To prevent accumulation over more than one heart cycle the integration of vessel wall velocity is restarted every heart cycle.

\subsection{Conclusion}

In the present chapter a method is described to estimate non-invasively the dimensionless arterial impedance. The method is based upon the evaluation of the spectral components of the normalized distension waveform and the normalized centerline blood flow velocity waveform, both obtained by means of ultrasound using off-line signal processing in three regions in depth (anterior vessel wall, center of the lumen, posterior vessel wall). The off-line processing is performed in the RF-domain and consists of an RF-domain velocity estimator preceded by a static vessel wall filter. It is demonstrated that for the harmonics between two and five and for Womersley numbers as observed in the common carotid artery center-line blood flow velocity is linear with 
volume flow. Moreover, the distension waveform is linearly related to the pressure waveform for a wide frequency range. Therefore, the spectral pressure-flow relationship may be replaced by the spectral distension-velocity relationship. It is concluded that in vitro for experimental considerations similar to the hemodynamic conditions in the aorta the proposed method to estimate dimensionless ultrasound arterial impedance gives the same results as the spectral pressure-flow relationship. 


\section{Chapter 8}

\section{In vivo validation of the assessment of dimensionless arterial impedance}

8.1 Abstract.

8.2 Introduction 128

8.3 Method of measurement. 129

8.4 Measurements 130

8.5 Results. 131

8.5 Discussion. 135

8.6 Conclusion 137 


\subsection{Abstract}

The structure of the arterial walls will change with age due to the fatiguing effects of the cyclic stress imposed on the walls by the pulsatile pressure in the arteries. The most important changes with age are dilation and stiffening of the artery walls and these effects will change the dynamic properties of the arterial system. The age related changes in the dynamic properties imply that arterial impedance and, hence, the shape and magnitude of the blood pressure and volume flow waveforms, will change with age. In vivo studies on the changes of arterial impedance with age in humans are scars, mainly because methods to assess non-invasively arterial impedance are lacking. In this Chapter the in vivo validation of the method to assess non-invasively dimensionless arterial impedance in the human carotid artery as described in Chapter 7 is presented. This validation concerns the assessment of the reproducibility of the method to determine dimensionless arterial impedance. Beside the effect of age on dimensionless arterial impedance was studied. Reproducibility was investigated by means of eight repeated measurements in the common carotid artery of twelve healthy volunteers. The age related effects were investigated in two age categories, i.e., 20-26 years $(n=4)$ and 62-69 years $(n=5)$. In agreement with the age related change in artery wall properties there is a significant change in the non-invasively estimated arterial impedance with age. The phase of the harmonics is higher in the older age category, while the magnitude of the harmonics is lower in this age category. The mean coefficient of variation over the total population for repeated measurements of arterial impedance (magnitude and phase) was $29 \%$, a high value.

Key words: age related change, center-line flow velocity, pulsed ultrasound, arterial impedance, vessel wall displacement.

\subsection{Introduction}

The subject of this Chapter is to validate in an in vivo study the non-invasive method to estimate dimensionless arterial impedance at different ages.

Arterial impedance as assessed at a given location in the arterial system is determined by the hemodynamic properties of the arterial system downstream of this location. It is defined as the ratio of blood pressure and blood volume flow for $n$ successive harmonics of heart rate (7.1), i.e., the base frequency. Randall and Stacy were the first to propose the concept of arterial impedance in blood vessels (Randall and Stacey 1956). So far, investigation of the properties of the arterial system by assessing arterial impedance has not been widely applied in medical diagnosis. The data on arterial impedance presently available are mainly based upon invasive measurements in animals (Randall and Stacey 1956, Attinger et al. 1966, Gow and Taylor 1968, Farrar et al. 1978), because studies on arterial impedance in humans are hampered by the lack of reliable non-invasive techniques to record simultaneously pressure and volume flow as a function of time locally. 
The method used in the present study to estimate non-invasively arterial impedance is based on the assessment of the ratio of the harmonics of the normalized change in diameter waveform (distension) and the harmonics of the normalized center-line mean blood flow velocity waveform during the cardiac cycle (7.2, Section 7.2). The observed distension and center-line blood flow velocity waveforms are normalized (Fig. 7.1) with respect to the peak-to-peak excursions of distension and of center-line blood flow velocity within a cardiac cycle, respectively. Normalization is used because then no assumptions have to be made for conversion of the distension waveform to a local blood pressure waveform as function of time in absolute units of pressure (Powalowski 1989). The consequence of this approach is that the arterial impedance obtained is dimensionless and that the shape of distension and center-line blood flow velocity waveforms are considered rather than the incidental magnitude of these waveforms.

In this Chapter the method to estimate arterial impedance as described in Chapter 7 will be applied to distension and center-line blood flow velocity waveforms as assessed with ultrasound in the common carotid artery of twelve healthy volunteers varying in age between 20 and 69 years. The reproducibility of this method to determine the dimensionless arterial impedance was studied by assessing consecutively eight distension and center-line blood flow velocity waveforms during a cardiac cycle. The interval between the assessments was on the order of two minutes. The wide range in age of the volunteers in this in vivo study was chosen to investigate also the changes in arterial impedance in two age categories (20-26, 62-69).

\subsection{Method of measurement}

The assessment of distension and center-line blood flow velocity is described in detail in Section 7.3.

A difference between the method of measurement as outlined in Section 7.3 and as used in this chapter is the application of an additional emitter/receiver unit, controlling the ultrasound probe of a conventional echo system once the ultrasound beam has been positioned correctly. The emission frequency of the additional emitter/receiver unit is 5 $\mathrm{MHz}$ and the sample frequency of the data acquisition system was set at $20 \mathrm{MHz}$. This additional emitter/receiver unit was used because conventional echo systems do not generate ultrasound bursts in echo M-mode at a pulse repetition frequency (PRF) in accordance with the anticipated blood flow velocities. The additional emitter/receiver provides an output of the RF-signal in combination with the trigger for emission and a synchronous clock signal.

The spatial estimation window (Fig. 2.5, Section 2.3.1) of the RF-domain crosscorrelation (CCM) estimator (Section 2.3.8) was selected according to the length of the emitted ultrasound burst in echo mode and was set at 8 sample points (sp), equivalent to $308 \mu \mathrm{m}$. The temporal length of the estimation window (Fig. 2.5 Section 2.3.1) of the 
CCM estimator was set at $10 \mathrm{~ms}$, equivalent to $64 \mathrm{sp}$ at a pulse repetition frequency of 6 $\mathrm{kHz}$.

The dimensionless arterial impedance follows from the ratio of the harmonics of the normalized distension waveform and the harmonics of the normalized center-line blood flow velocity waveform during the cardiac cycle. The dimensionless arterial impedance was analyzed between the second to the fifth harmonic (Fig. 8.1) because in this range center-line flow velocity is almost linear with volume flow (Fig. 7.5, Section 7.4). Moreover, the lower harmonics of distension and center-line blood flow velocity contain almost all of the total signal power (Fig. 7.6).

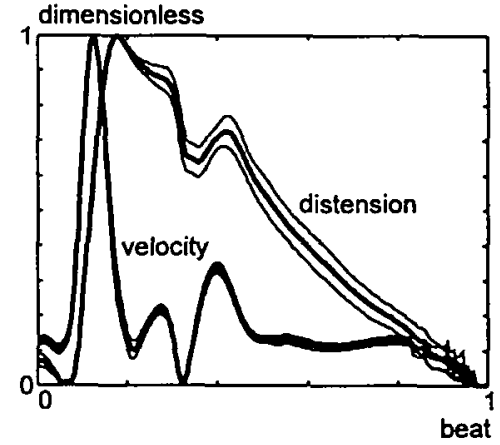

(a)

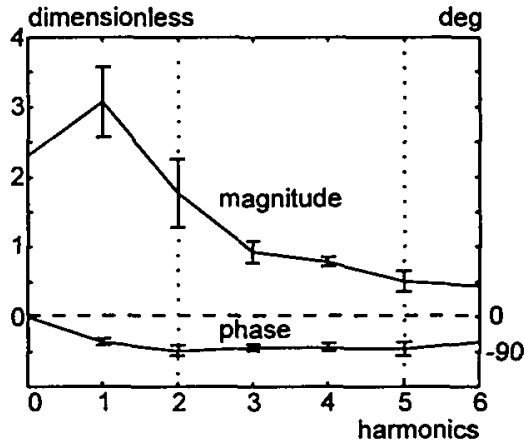

(b)

Fig. 8.1: (a) The mean (thick lines) and standard deviation (thin lines) of the normalized distension and center-line blood flow velocity of eight repeated measurements in the common carotid artery. (b) The magnitude and phase of the dimensionless arterial impedance as derived from the normalized waveforms depicted in (a).

\subsection{Measurements}

Twelve male, presumed healthy volunteers, raging in age between 20 and 69 years, were recruited. The volunteers were normotensive and had no evidence of cardiovascular disease. From all volunteers written informed consent was obtained. The research protocol was approved by the joint ethical committee of the University Hospital Maastricht and the University of Limburg.

Data collection started after an adaptation period of 10 to 15 minutes in a supine resting position in an acclimatized room $\left(22^{\circ} \mathrm{C}\right)$. The common carotid artery was visualized in B-mode (ATL Mark IV, $5 \mathrm{MHz}$ mechanical sector scanner, Advanced Technology Labs, Bothell, WA, USA) with the head tilted in the contralateral direction. Using the tip of the flow divider as a landmark the probe position was manipulated until a suitable line of sight could be selected. It had to cross the carotid artery at an angle of $60^{\circ}$ or $70^{\circ}$ degrees, 2 to $3 \mathrm{~cm}$ proximal of the tip of the flow divider. After positioning the M-line the ultrasound system was switched to M-mode and a pulsed Doppler registration with 
the sample volume located midstream was made. The observed peak systolic velocity was noted: Subsequently the external emitter/receiver was activated and an RF registration was acquired, where acquisition commenced synchronously with a trigger derived from the R-top of the ECG. The registration was random with respect to the phase of the respiration cycle. Before accepting a measurement a quick check of the acquired data was executed. A basic requirement for acceptance was agreement of the peak systolic center stream velocities obtained with the conventional pulsed Doppler system and with the arterial impedance system. Because the former system gives the spectral envelope and the latter system the mean velocity, it was acceptable that the latter system produced a lower mean velocity but it should not be less than $80 \%$ of the value obtained with the former system. Based on the criteria listed above on the order of $20 \%$ of the registrations had to be excluded because of excessive RF interference or motion artefacts (movements of the artery with respect to the probe). To obtain eight valid measurements on the order of ten measurements were actually made ${ }^{1}$. While the data were checked for velocity consistency the ultrasound probe was removed from the subject. Therefore, a subsequent registration for reproducibility measurements required exact replacement of the probe in the same position and orientation.

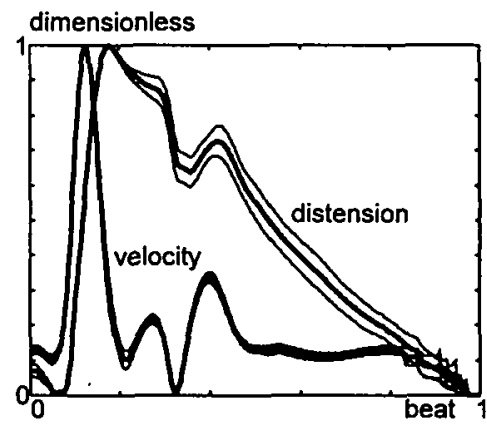

(a)

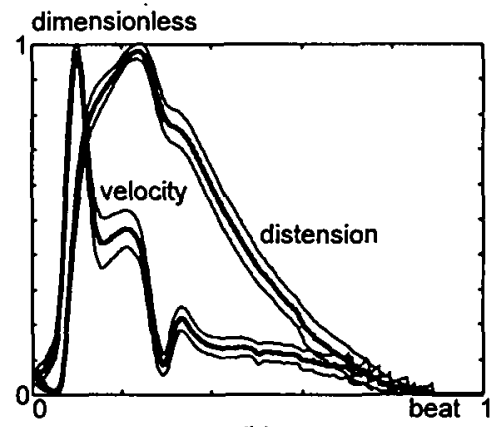

(b)

Fig. 8.2: The mean (thick lines) and standard deviation (thin lines) of the normalized distension and center-line blood flow velocity of eight repeated measurements on a young (age: $26 \mathrm{yrs)}$ healthy volunteer (a) and an old (age: 65 yrs) healthy volunteer (b). Note difference in shape of the velocity and distension waveform between the young and the old volunteer.

\subsection{Results}

In each volunteer of the two subject populations eight repeated measurements of the distension and center-line blood flow velocity waveforms during one cardiac cycle were made. Moreover, the end-diastolic diameter, the peak to peak change in diameter and the peak systolic center-line blood flow velocity were measured. Table 8.1 contains the subject population (healthy volunteers) in consecutive order of age, their age, of eight repeated measurements the mean end-diastolic diameter $D$, the mean distension $\Delta d$, the

\footnotetext{
I With acknowledgements to Steven Samijo, who did all these examinations
} 
mean peak systolic center-line blood flow velocity $v_{c}$ over one cardiac cycle and the mean for both age categories (20-26 yrs, 62-69 yrs).

Table 8.1: The mean hemodynamic properties of eight repeated measurements in the common carotid artery of 12 healthy subjects with in between parentheses the coefficient of variation as a percentage.

\begin{tabular}{c|cccc} 
Subject & age & $D \mathrm{~mm}$ & $\Delta d \mu \mathrm{m}$ & $v_{\mathrm{c}} \mathrm{mm} / \mathrm{s}$ \\
\hline hd7 & 20 & $6.3(4.8)$ & $735(17)$ & $1090(6)$ \\
fh7 & 21 & $6.2(1.6)$ & $861(13)$ & $920(11)$ \\
sw7 & 23 & $7.1(2.8)$ & $908(8)$ & $1120(10)$ \\
lk6 & 26 & $6.0(3.3)$ & $610(20)$ & $930(4)$ \\
sk5 & 36 & $6.1(4.9)$ & $405(12)$ & $961(8)$ \\
pe5 & 44 & $6.4(8.3)$ & $513(8)$ & $680(9)$ \\
jr3 & 59 & $6.3(3.2)$ & $302(24)$ & $687(10)$ \\
mg3 & 62 & $7.6(3.9)$ & $246(23)$ & $637(12)$ \\
cp3 & 65 & $7.4(6.8)$ & $396(25)$ & $609(10)$ \\
j12 & 66 & $6.8(5.9)$ & $301(19)$ & $512(11)$ \\
nh2 & 67 & $7.5(1.3)$ & $356(7)$ & $619(13)$ \\
pb2 & 69 & $7.3(2.7)$ & $589(13)$ & $464(7)$ \\
\hline mean & $20-26$ & $6.4(3.1)$ & $779(14)$ & $1015(8)$ \\
mean & $62-69$ & $7.3(4.1)$ & $378(17)$ & $568(11)$
\end{tabular}

The internal end-diastolic diameter could be assessed with a low coefficient of variation, ranging from 1.3 to $8.3 \%$. The peak to peak change in arterial diameter shows a large coefficient of variation, ranging from 8 to $25 \%$. Peak systolic center-line blood flow velocity measurements could be made with a lower coefficient of variation, ranging from 4 to $13 \%$. As could be expected all three variables (diameter, peak to peak diameter change and peak systolic center-line velocity) showed a significant variation in both age categories (student's t-test). Arterial diameter is larger, while distension and center-line velocity were smaller and lower, respectively, in the older age category. The significant changes with age also applied to the shape of the distension and center-line velocity waveforms as shown in Fig. 8.3. From each of the eight repeated measurements (distension and center-line velocity) per subject a dimensionless arterial impedance was estimated. So a mean and standard deviation per harmonic value of the dimensionless arterial impedance per subject could be computed. However, only the second to the fifth harmonic values were considered in analysing the dimensionless arterial impedance because in this range the center-line blood velocity waveform is linear with the volume flow waveform (Section 7.4). In table 8.2 and 8.3 are presented the subjects in consecutive order of age, their age, of eight repeated measurements the mean harmonic values in the range between harmonic two to five $\left(h_{2}\right.$ to $\left.h_{5}\right)$ of magnitude and phase, respectively. Moreover, the mean for both age categories (20-26 yrs, 62-69 yrs). 
Table 8.2: The mean of 8 repeated measurements of the magnitude per harmonic of the dimensionless arterial impedance of 12 healthy subjects with in between parentheses the coefficient of variation as a percentage.

\begin{tabular}{c|ccccc} 
Subject & age & $\mathrm{h}_{2}$ & $\mathrm{~h}_{3}$ & $\mathrm{~h}_{4}$ & $\mathrm{~h}_{\mathrm{s}}$ \\
\hline hd7 & 20 & $1.3(33)$ & $1.1(18)$ & $0.9(33)$ & $0.6(33)$ \\
fh7 & 21 & $1.5(20)$ & $1.0(10)$ & $0.8(25)$ & $0.6(16)$ \\
sw7 & 23 & $1.3(23)$ & $0.9(11)$ & $0.7(14)$ & $0.5(20)$ \\
lk6 & 26 & $1.8(27)$ & $0.9(22)$ & $0.8(13)$ & $0.5(20)$ \\
sk5 & 36 & $0.7(42)$ & $0.9(11)$ & $0.6(50)$ & $0.7(28)$ \\
pe5 & 44 & $1.5(20)$ & $1.1(18)$ & $0.8(13)$ & $0.5(20)$ \\
jr3 & 59 & $1.0(30)$ & $0.8(37)$ & $0.7(57)$ & $0.5(60)$ \\
mg3 & 62 & $1.1(27)$ & $0.7(28)$ & $0.7(57)$ & $0.6(66)$ \\
cp3 & 65 & $1.1(18)$ & $0.9(33)$ & $0.7(43)$ & $0.5(20)$ \\
j12 & 66 & $1.1(36)$ & $1.1(36)$ & $0.9(44)$ & $0.7(43)$ \\
nh2 & 67 & $1.2(8)$ & $0.9(11)$ & $0.5(20)$ & $0.5(20)$ \\
pb2 & 69 & $0.7(28)$ & $0.7(29)$ & $0.7(57)$ & $0.5(40)$ \\
\hline mean & $20-26$ & $1.5(26)$ & $1.0(15)$ & $0.8(21)$ & $0.6(22)$ \\
mean & $62-69$ & $1.0(23)$ & $0.9(27)$ & $0.7(44)$ & $0.6(38)$
\end{tabular}

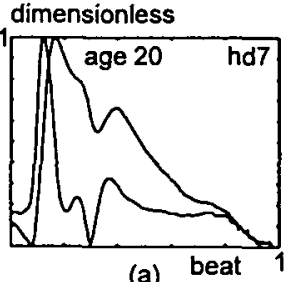

(a)

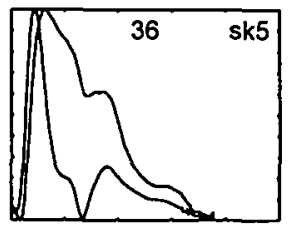

(e)

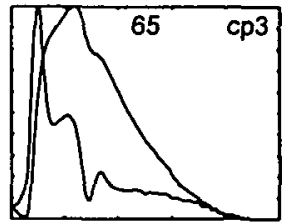

(i)

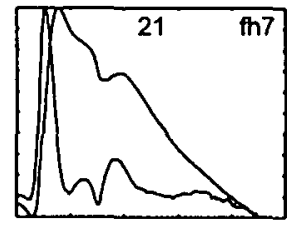

(b)

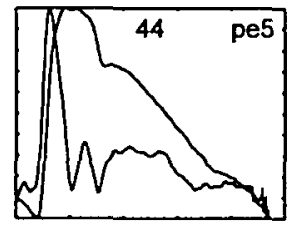

(f)

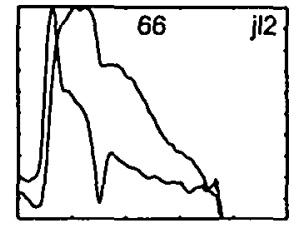

(j)

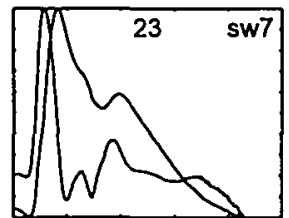

(c)

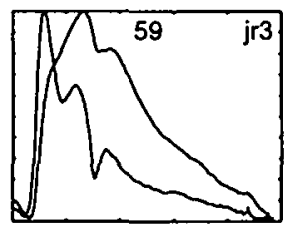

(g)

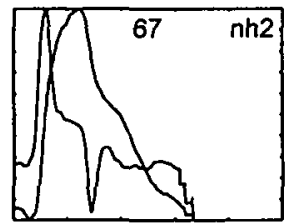

(k)

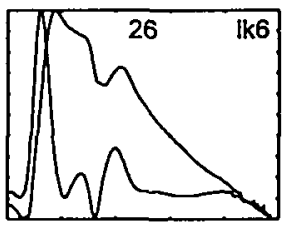

(d)

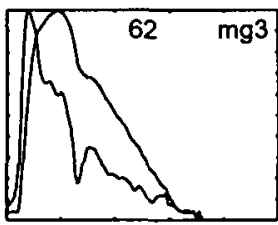

(h)

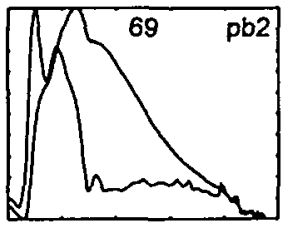

(l)

Fig. 8.3: The normalized distension and center-line blood flow velocity waveforms of the subject population in consecutive order of age (20 to $69 \mathrm{yrs})((\mathrm{a}),(\mathrm{b}), \ldots,(\mathrm{l}))$. 
Table 8.3: The mean of 8 repeated measurements of the phase (radials) per harmonic of the dimensionless arterial impedance of 12 healthy subjects with in between parentheses the coefficient of variation as a percentage.

\begin{tabular}{c|ccccc} 
Subject & age & $\mathrm{h}_{2}$ & $\mathrm{~h}_{3}$ & $\mathrm{~h}_{4}$ & $\mathrm{~h}_{5}$ \\
\hline hd7 & 20 & $-1.4(14)$ & $-1.3(8)$ & $-1.3(15)$ & $-1.3(23)$ \\
$\mathrm{fh7}$ & 21 & $-1.4(14)$ & $-1.3(8)$ & $-1.3(8)$ & $-1.3(15)$ \\
sw7 & 23 & $-1.4(14)$ & $-1.3(15)$ & $-1.4(14)$ & $-1.4(14)$ \\
lk6 & 26 & $-1.5(13)$ & $-1.4(14)$ & $-1.4(14)$ & $-1.4(21)$ \\
sk5 & 36 & $-1.0(20)$ & $-0.9(22)$ & $-0.9(33)$ & $-0.6(17)$ \\
pe5 & 44 & $-1.5(13)$ & $-1.2(17)$ & $-1.4(7)$ & $-1.2(17)$ \\
jr3 & 59 & $-1.1(27)$ & $-0.9(33)$ & $-1.2(50)$ & $-0.6(100)$ \\
mg3 & 62 & $-0.9(33)$ & $-0.9(44)$ & $-1.0(40)$ & $-0.8(50)$ \\
cp3 & 65 & $-1.1(18)$ & $-1.1(27)$ & $-1.3(38)$ & $-1.2(42)$ \\
j12 & 66 & $-1.1(36)$ & $-0.9(44)$ & $-1.1(82)$ & $-1.8(33)$ \\
nh2 & 67 & $-1.3(15)$ & $-1.6(19)$ & $-1.3(31)$ & $-1.3(15)$ \\
pb2 & 69 & $-1.2(17)$ & $-0.8(25)$ & $-0.8(63)$ & $-0.6(116)$ \\
\hline mean & $20-26$ & $-1.4(14)$ & $-1.3(11)$ & $-1.4(13)$ & $-1.4(18)$ \\
mean & $62-69$ & $-1.1(24)$ & $-1.1(32)$ & $-1.1(51)$ & $-1.1(51)$
\end{tabular}

The magnitude and phase of the second harmonic of the dimensionless arterial impedance were significantly different between both age categories. The magnitude and phase of the third, fourth and fifth harmonics of the dimensionless arterial impedance were not significantly different between both age categories.

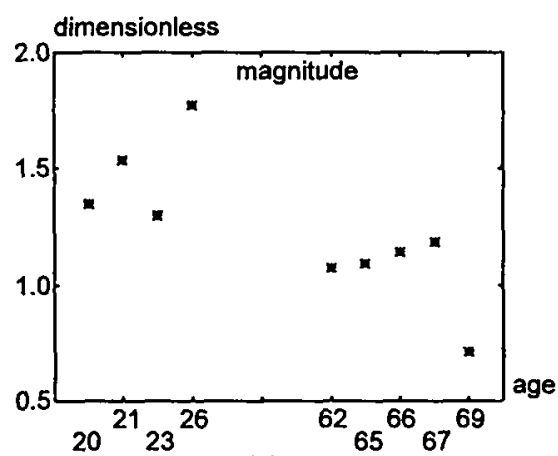

(a)

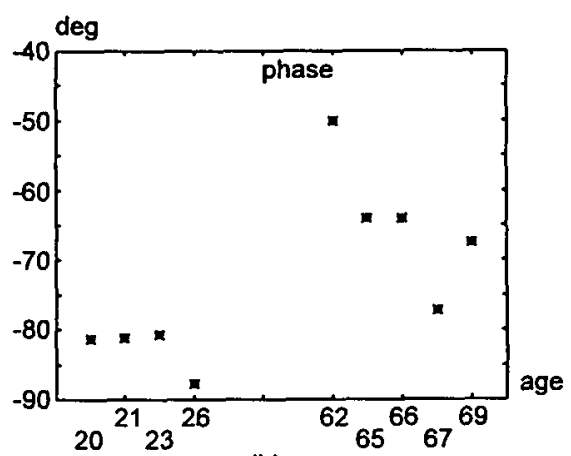

(b)

Fig. 8.4: The mean magnitude (a) and mean phase (b) of the second harmonic of the dimensionless arterial impedance for both age categories.

The mean coefficient of variation of the harmonics of magnitude and phase was $29 \%$. The coefficient of variation of magnitude and phase per harmonic of the dimensionless arterial impedance was $25 \%$ and $20 \%$ for the second harmonic, $22 \%$ and $23 \%$ for the third harmonic, $36 \%$ and $33 \%$ for the fourth harmonic and $32 \%$ and $39 \%$ for the fifth harmonic, respectively. The second and third harmonics show a significantly lower value for the coefficient of variation than the fourth and fifth harmonics. All the coefficients of variation are very high indicating a poor reproducibility between the 
independent estimates of the dimensionless arterial impedance. On the other hand the coefficient of variation in the in vitro study was only $4 \%$, which is an indication of a good reproducibility (Section 7.5.3).

The harmonic values of the subject population in consecutive order of age are shown in Fig. 8.5. In this figure ten harmonic values are shown although only for the second to the fifth harmonic values liniarization between center-line blood flow velocity and volume flow is allowed (Section 7.4).

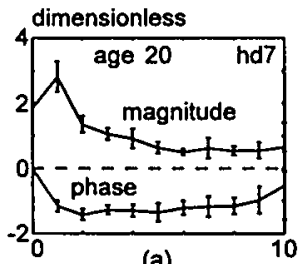

(a) harmonics

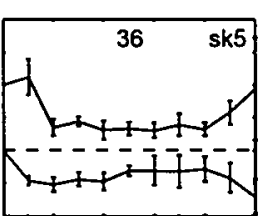

(e)

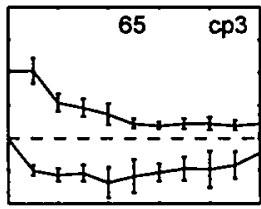

(i)

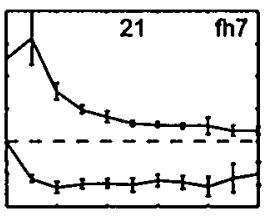

(b)

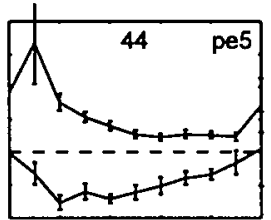

(f)

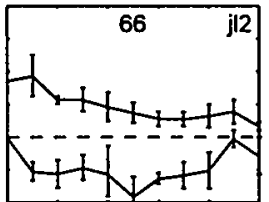

(j)

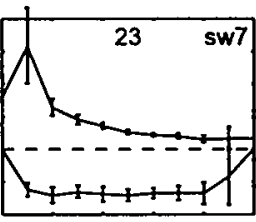

(c)

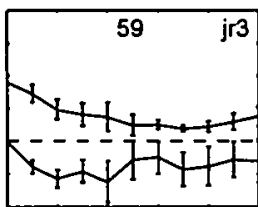

(g)

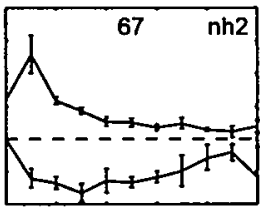

(k)

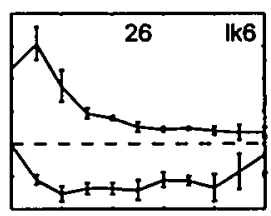

(d)

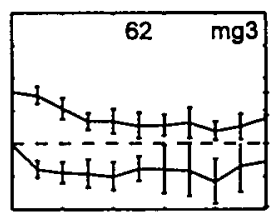

(h)

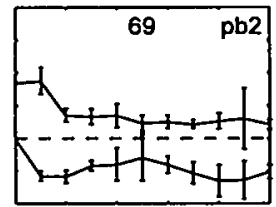

(I)

Fig. 8.5: Mean harmonic values of eight repeated measurements of magnitude and phase of the dimensionless arterial impedance in the consecutive order of age (20 to $69 \mathrm{yrs})$ ((a),(b),...(I)). The bars indicate the standard deviations.

\subsection{Discussion}

The findings in the present non-invasive validation study show that arterial impedance can be assessed without the measurement of local blood pressure. The dimensionless arterial impedance was determined from the ratio of the harmonics of the normalized distension waveform and the normalized center-line blood flow velocity waveform. This dimensionless arterial impedance was determined for the second through the fifth harmonic because in the common carotid artery in this range the center-line blood flow velocity is almost linear with volume flow (Section 7.4). The mean coefficient of variation of the dimensionless arterial impedance for eight repeated measurements in one cardiac cycle in the range of harmonics considered was $29 \%$. In the clinical study of 
Powalowski ( 8 repeated measurements in 43 healthy volunteers varying in age between 9 and 64 years) the overall mean coefficient of variation in the windkessel model parameters used to analyse the arterial impedance was $24 \%$ (Powalowski 1989). The second and third harmonic values have a significantly lower coefficient of variation than the fourth and fifth harmonic values. The reason for this significant difference is the decreasing level in signal power for increasing harmonics (Fig. 7.6). The high coefficient of variation in the repeated measurements is an indication of a poor reproducibility of the dimensionless arterial impedance. However, a validation of the proposed method by means of the coefficient of variation of repeated measurements is disputable because this variation is not only determined by variations in the method but also by as yet unknown physiological beat to beat variations in impedance. The latter is supported by the small coefficient of variation $(4 \%)$ as found in the ten repeated measurements of dimensionless arterial impedance in the in vitro study (Section 7.5). A possible solution to reduce the beat to beat variation is to estimate the dimensionless arterial impedance from a number of averaged beats. As suggested by Murgo the minimum number of beats to be averaged for reliable results should be 10 (Murgo et al. 1980). However, the beat to beat variation in the shape of the distension and center-line blood flow velocity waveforms is not a normally distributed variation. So averaging will not give a reduction in the beat to beat variation. Another solution to reduce beat to beat variation is to average the spectra of a number of distension and center-line velocity waveforms having a beat to beat cross-correlation coefficient greater than a given threshold. The latter method was used by Chon with a threshold of $99.5 \%$ to estimate hemodynamic parameters from tonometer blood pressure recordings (Chon et al. 1995).

An additional purpose of this in vivo study was to investigate the differences in dimensionless arterial impedance, if any, in two categories of age. It has been known from several studies (Pagani et al. 1979, Reneman et al. 1985, Reneman et al. 1986) that ageing will change the dynamic properties of the arterial walls probably due to structural changes in the vessel walls. As could be expected the hemodynamic variables diameter, peak to peak distension and the peak systolic center-line blood flow velocity were significantly different in the two categories of age (Section 8.5). The diameter of the common carotid artery is larger in the old category, while peak to peak distension is smaller and peak systolic center-line blood flow velocity is lower in the old category. The increase in diameter of the artery allows the arteries to maintain their cushioning effect, despite a decrease in distension. The decrease in peak systolic center-line blood flow velocity at older age can at least partly be explained by an increase in diameter, but changes in stroke volume can not be excluded. The significant change in the shape of the distension and center-line blood flow velocity waveforms with age (Fig. 8.3) probably results from an increase in arterial impedance with age. However, this change was only significant for the second harmonic and not for the third to the fifth harmonics of the dimensionless arterial impedance. The magnitude of the second harmonic value is smaller, while the phase of the second harmonic is larger in the older age category. The meaning of this age related change in the dimensionless arterial impedance is that the temporary blood storage capacity of the vascular bed downstream of the common 
carotid artery is lower for the older age category despite of the increase in diameter for this age category. The change in phase with age is in agreement with clinical study of Powalowski but the change in magnitude with age was not found in this study (Powalowski 1989). The conclusion of Powaloski was that it was inertia of the blood volume in the arterial system which was age related and not the temporary blood storage capacity. However, this is not in agreement with the increase in arterial diameter with age because an increase in diameter will give an decrease in inertia of the blood volume.

The application of harmonic analysis to the distension and center-line velocity waveforms implies that the heart rate is regular so that the arterial system is in a steady state of oscillation. If the heart rate is irregular it might appear that the application of harmonic analysis is invalid. This is the reason to restrict the harmonic analysis to waveforms of one heart beat only or to a number of averaged waveforms of one heart beat with a high cross-correlation coefficient.

\subsection{Conclusion}

Non-invasive ultrasound techniques can be used to assess the dimensionless arterial impedance in the common carotid artery from the ratio of the harmonics of the normalized distension and center-line blood flow velocity waveforms. Normalization is performed with respect to the peak to peak excursions of both waveforms because then no assumptions have to be made for the conversion of the distension waveform to local blood pressure in absolute units of pressure. Estimation of dimensionless arterial impedance employing ultrasound is possible because distension is linear with pressure. Moreover, for the second to the fifth harmonics and for a Womersley number as observed in the common carotid artery center-line blood flow velocity is linear with volume flow. The in vivo validation study shows that the dimensionless arterial impedance can be assessed with a mean coefficient of variation for the second to the fifth harmonics of $29 \%$. Moreover, the second harmonic of the dimensionless arterial impedance appears to be age related. The third to fifth harmonics did not show a significant change with age. The age related changes showed that frequency damping (lower magnitude) is higher in the old age category and that the temporary blood storage capacity (higher phase) is lower in this age category. These age related effects on the dimensionless arterial impedance are in agreement with studies on the age related effects on the arterial vessel walls. It can be concluded that the proposed method to estimate non-invasively dimensionless arterial impedance in vivo has a high coefficient of variation for repeated measurements. It remains to be explored whether this large variation is also determined by physiological variation in arterial impedance. 



\section{Chapter 9}

\section{General discussion}

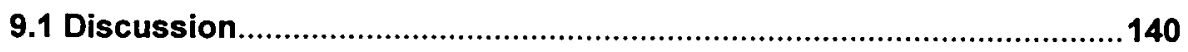

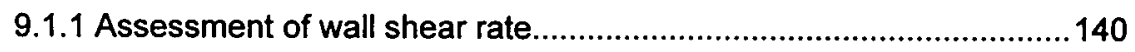

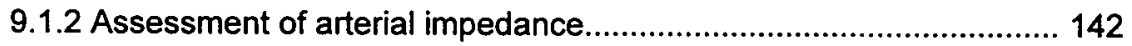

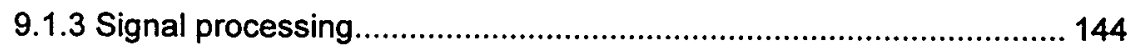

9.2 Conclusions

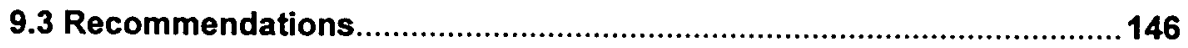




\subsection{Discussion}

The aim of this thesis was the development of acquisition and signal processings methods for the non-invasive assessment of wall shear rate and arterial impedance by means of ultrasound. Wall shear rate is the near wall velocity gradient, while arterial impedance is defined as the ratio of the spectral components of the local blood pressure waveform and the local blood volume flow waveform during the cardiac cycle. In the study of hemodynamic parameters non-invasive assessment is preferred because then the physiological conditions of the parameter(s) to be measured are not affected, while human studies became feasible. The non-invasive assessment of wall shear rate and arterial impedance by means of ultrasound has its limitations. Inadequate spatial resolution of ultrasound is considered to be a major drawback in the assessment of wall shear rate, because of the steep slope of the velocity profile near the vessel wall. An even more important limitation of ultrasound is its inability to discriminate unambiguously between reflectors induced by slowly moving structures, like vessel walls, and scattering induced by slowly moving blood near the vessel wall because the amplitude of the reflections is on the order of $40 \mathrm{~dB}$ higher than the amplitude of the scattering (Section 3.3.1). To overcome this limitations radio frequency domain (RFdomain) signal processing algorithms applied to ultrasound RF-signals acquired in motion mode (M-mode) or Doppler-mode were developed (Chapter 5). M-mode is preferable above Doppler-mode because of its high spatial resolution (short ultrasound emission burst).

A major limitation in the non-invasive assessment of arterial impedance is the difficulty to simultaneously record pulsatile blood pressure and blood volume flow at the same location. To overcome this limitation the local distension (change in diameter) waveform, is used as a substitute for the local blood pressure waveform, while the local center-line blood flow velocity waveform is used as a substitute for the local blood volume flow waveform. Both waveforms can simultaneously and accurately be assessed by means of ultrasound. The ratio of the spectral components of the distension waveform and the center-line blood flow velocity waveform provides an estimate of arterial impedance.

\subsubsection{Assessment of wall shear rate}

There is increasing evidence that wall shear rate and/or stress interacts functionally with the arterial wall and that these physical factors may play a role in the origin of vessel wall anomalies. The role of wall shear rate, however, is as yet incompletely understood, mainly because no methods were available to assess non-invasively this mechanical factor in vivo. To determine accurately wall shear rate from velocity profiles, one must be able to assess unambiguously low blood flow velocities near the vessel wall. These velocities can be determined accurately by means of a cross-correlation model (CCM) estimator (Section 2.3.8) preceded by an adaptive discrimination filter (ADP-f, Section 4.4.5) acting in the radio frequency domain. The adaptive filter acts as a band-pass filter 
with the central frequency of rejection adapted to the mean velocity of vessel wall motion or structure motion. In this way a maximum suppression of the reflections with a minimum width of the rejection range is achieved (Fig 4.3, Section 4.4.5). Normally a static discrimination filter is used to discriminate between reflections and scattering. In this type of filter, however, the time dependent aspect of the reflections is ignored (vessel wall motion). A great advantage of the adaptive filter method over a static echo removal filter is that the rejection range can be kept small (Section 3.3.2) and that the low (near wall) blood flow velocities can be assessed accurately. Therefore, the velocity estimation range is substantially greater for the CCM estimator preceded by an adaptive filter than for the CCM estimator preceded by a static filter (Section 4.6).

The limitation in spatial resolution $(250 \mu \mathrm{m})$ of ultrasound restricts the accuracy of the assessment of the shear rate near the wall. Therefore, the maximum velocity gradient is used as an estimate of wall shear rate at both the anterior and the posterior vessel wall lumen interface (Fig. 5.3) rather than the near wall velocity gradient. To obtain an estimate of the axial wall shear rate the maximum velocity gradients at the anterior and posterior vessel walls are averaged. This is only valid for a relatively straight vessel like the common carotid artery because only then the radial velocity components at the anterior and posterior vessel wall are almost equal and opposite in direction.

The validation of the method to estimate wall shear rate was performed in two in vivo studies (Chapter 5, Chapter 6) and one in vitro study (Chapter 6). The in vivo study in Chapter 5 concerns a pilot study on the reproducibility of peak wall shear rate assessment in comparison with the reproducibility of peak center-line blood flow velocity assessment ( 6 repeated measurements in the common carotid artery of 5 young healthy volunteers). The coefficient of variation of peak wall shear rate was $5 \%$, while the coefficient of variation of peak center-line blood flow velocity was $9 \%$. The in vivo study in Chapter 6 concerns the comparison and reproducibility of mean wall shear rate in two subject populations of different ages $(20-30$ years, $n=9 ; 60-70$ years, $n=7)$. The mean coefficient of variation for repeated measurements of mean wall shear rate was $13 \%$. The in vitro study concerned a comparison of the velocity distribution and wall shear rate assessed with both ultrasound and laser Doppler anemometry (LDA) in a distensible tube under non-stationary flow conditions, the flow pattern in the tube resembling that in the descending aorta. The mean normalized root mean square (RMS) difference in wall shear rate waveform assessment between the two methods was $9.4 \%$. This value indicates good accuracy of the wall shear rate assessment by means of ultrasound because the flow velocity distributions as assessed with LDA are based on ensemble averaging of 32 repetitive measurements in time and the flow velocity distribution as assessed with ultrasound is based on only one single recording.

The coefficient of variation of the peak wall shear rate in the in vivo study of Chapter 5 was $5 \%$, while the coefficient of variation of the mean wall shear rate in the in vivo study of Chapter 6 was 13\%. The difference in the coefficient of variation in the different quantities (peak wall shear rate and mean wall shear rate) for both the in vivo 
studies can be explained from the difference in post-processing (Section 6.2) and the use of an additional emitter/receiver in the in vivo study of Chapter 6. In the first version of the post-processing of the method to estimate wall shear rate the velocity distribution was subjected to a smoothing filter to remove the quantisation noise introduced by the median filter (Fig. 5.6, Section 5.5). This smoothing filter will reduce the spatial resolution of the velocity distribution giving an underestimation of wall shear rate. The smoothing filter was omitted in the in vivo study of Chapter 6 . The use of an additional emitter/receiver unit circumvents the practical limitations of commercially available echo-systems but has several drawbacks. First the additional cable attachment promotes RF interference and noise sensitivity. The practical problems associated with the system configuration can only be eliminated if echo-systems support a sufficiently high pulse repetition frequency in echo M-mode (for peripheral applications exceeding $10 \mathrm{kHz}$ ) in combination with a sample clock that is phase locked to the trigger for emission. Systems of this kind are not (commercially) available yet. In the pilot study of Chapter 5 a prototype of a color flowmapper (developed at the department of Biophysics, University of Limburg) was used. This system supports a sufficiently high pulse repetition frequency in echo $\mathrm{M}$-mode $(8 \mathrm{kHz})$ in combination with a phase locked sample clock, but this system can not be used in a clinical environment.

In agreement with the assumed autoregulation of wall shear stress, both categories of age exhibited the same mean wall shear rate of $340 \mathrm{~s}^{-1}$ (Chapter 6). Assuming a blood viscosity of $3.5 \mathrm{mPa} . \mathrm{s}$, a mean wall shear rate of $340 \mathrm{~s}^{-1}$ corresponds with a mean wall shear stress of $1.2 \mathrm{~Pa}$, which is somewhat lower than the shear stresses reported in the literature (Rodbard 1975, Kamiya and Togawa 1980, LaBarbera 1990). A possible explanation for the higher shear stresses reported in the literature is that these were computed from volume flow models in which the elastic behavior of the artery wall was ignored. As demonstrated with computer models (Perktold et al. 1994) and in in vitro experiments (Duncan et al. 1990) the elastic behavior of the artery wall results in a reduction of peak wall shear rate, which is in agreement with our in vivo results. However, underestimation of the wall shear rate values as assessed by means of ultrasound can not be excluded.

The discrimination filter necessary (Chapter 3 ) in the ultrasound signal processing will introduce an absolute minimum in the assessment of wall shear rate. A temporal estimation window of $10 \mathrm{~ms}$ limits the minimum width of the wall rejection filter to 100 $\mathrm{Hz}$ (Section 3.3.2), centered at the temporal mean frequency of the clutter signals, which is close to zero in the diastolic phase. A cut-off frequency of $50 \mathrm{~Hz}$ is equivalent to an absolute minimum flow velocity of $22 \mathrm{~mm} / \mathrm{s}$ or an absolute wall shear rate of $70 \mathrm{~s}^{-}$ 1 at the wall lumen boundary (transition from zero flow velocity to a valid velocity). However, all in vivo registrations made showed a minimum value at end-diastole which were higher than the critical one, indicating that the velocity gradient was derived from the slope rather than from the edge of the instantaneous velocity profile (Section 6.4.1). 


\subsubsection{Assessment of arterial impedance}

Assessment of arterial impedance is, for example, important when investigating the load exposed on the left ventricle or to appreciate the hemodynamic consequences of stenoses and occlusions of arteries. However, pulsatile pressure and volume flow waveforms, simultaneously recorded at the same location, are difficult to obtain noninvasively. The non-invasive assessment of arterial impedance as described in this thesis is based on the substitution of the pressure waveform by the distension waveform and the volume flow waveform by the center-line blood flow velocity waveform (Section 7.2). This substitution is allowed if the distension waveform and the center-line blood flow velocity waveform are linearly related to the blood pressure waveform and the volume flow waveform, respectively. In chapter 7 it is shown that in the common carotid artery for frequency components between two and five $\mathrm{Hz}$ the center-line blood flow velocity waveform is almost linear with the volume flow waveform and that the distension waveform is linear with the pressure waveform over a wide frequency range. Moreover, this liniarization was validated in an in vitro study (Section 7.5). The ratio of the frequency components of the normalized distension waveform during the cardiac cycle and those of the normalized center-line blood flow velocity waveform gives an estimate of the dimensionless arterial impedance. Normalization of both waveforms is necessary to avoid the conversion of the distension waveform to absolute units of pressure $(\mathrm{mmHg})$ and of the center-line blood flow velocity to absolute units of volume flow $(1 / \mathrm{min})$. However, due to linearization and normalization it is impossible to estimate peripheral resistance.

The in vivo validation of the method to estimate arterial impedance involved the reproducibility of arterial impedance. Besides, the effect of age on arterial impedance was studied. Reproducibility was investigated by means of eight repeated measurements in the common carotid artery of twelve healthy volunteers, varying in age between 20 and 69 years. The age related effects were investigated in two age catogories, i.e., 20-26 years $(n=4)$ and $62-69$ years $(n=5)$.

The mean coefficient of variation of the dimensionless arterial impedance for eight repeated measurements of one cardiac cycle in the considered range of harmonics (two to five) was $29 \%$. The high coefficient of variation in the repeated measurements is an indication of a poor reproducibility of the dimensionless arterial impedance. However, a validation of the proposed method by means of the coefficient of variation of repeated measurements is disputable because this variation is not only determined by variations in the method but also by as yet unknown physiological beat to beat variations in impedance. The latter is supported by the small coefficient of variation (4\%) as found in ten repeated measurements of dimensionless arterial impedance in the in vitro study (Section 7.5).

In agreement with the age related change in arterial wall properties there was a significant change with age in the non-invasively estimated dimensionless arterial 
impedance. However, this change was only significant for the second harmonic and not for the third to the fifth harmonics of the dimensionless arterial impedance. The magnitude of the second harmonic value is smaller, while the phase of the second harmonic is larger in the older age category. This age related change in the dimensionless arterial impedance can be explained by the lower temporary blood storage capacity of the vascular bed downstream of the common carotid artery in the older age category.

\subsubsection{Signal processing}

Ultrasound signal processing is the extraction of information from the information carriers in the radio frequency signals (RF-signals) received. The carriers of information in the RF-signals received are reflections from structures and scattering from red blood cells. In general the ultrasound signal processing consists of a filter, for the discrimination between reflections and scattering, and a mean frequency estimator for the estimation of the temporal mean frequency. The latter is directly related to the mean blood flow velocity by means of the Doppler equation.

An important part of the ultrasound signal processing is the acquisition of RF-signals received. To retain all (spatial) information available in the ultrasound signals returned by reflection and scattering it is essential to capture the radio-frequency signals at a point close to the receiver of the ultrasound system. This allows for capturing of RFsignals with a wide bandwidth, concomitant with short ultrasound emission burst covering a few RF-periods. Conventionally, only in M-mode short ultrasound emission burst are employed. This restriction has some repercussions for the control of the ultrasound system in relation to the acquisition of RF-signals (Section 6.2). On the other hand the intermittent storage of the RF-signals in computer memory allows for iterative evaluation of RF-data to develop an optimal method for motion and velocity assessment.

The mean frequency estimators for ultrasound radio or low frequency signals (RF- or LF-signals) show an evolution from LF-domain parametric estimators to RF-domain parametric estimators. The reason for this shift is that in the LF-domain the random spatial distribution of scatterers is discarded in the demodulation process (Section 1.4.5). In the RF-domain this spatial information is retained. Conventionally, LFdomain mean frequency estimators are used to estimate blood flow velocity and/or tissue motion. The disadvantage of these estimators is that only the temporal mean frequency is estimated and that the spatial mean frequency is assumed to be constant over depth. In practice the frequency dependent attenuation will produce a gradual downward shift in spatial mean frequency. This effect will be more prominent for wide band ultrasound signals (short bursts) as employed in echo M-mode. To avoid the bias introduced by the assumption of a constant spatial mean frequency and to avoid the loss of the random spatial distribution of scatterers, RF-domain signal processing was employed for the assessment of blood flow velocity and tissue motion. 
Most of the mean frequency estimators are single mean frequency estimators. The latter are capable to assess the mean frequency of only one single signal component (reflection or scattering). However, RF- and LF-signals are composed of three major signal components: reflections, scattering and noise. So, single mean frequency estimators have to be preceded by discrimination filters to discriminate between the different signal components, whereafter the mean frequency estimator can estimate the mean frequency of the selected signal components. The main problem in the design of a discrimination filter is the overlap in temporal frequency and the great difference in amplitude $(40 \mathrm{~dB})$ between the clutter (reflections and reverberations) and the scattered signal components. The overlap in frequency makes it impossible to discriminate with a frequency selective filter without loss of information in the low frequency range (Section 3.2.1). The great difference in amplitude will introduce an even greater loss of information than the overlap in frequency. To reduce the loss of information caused by the great difference in amplitude an adaptive band-pass filter with a central frequency adapted to the temporal mean frequency of the clutter is introduced (Section 4.4.5), allowing the assessment of near wall blood flow velocity.

\subsection{Conclusions}

Non-invasive ultrasound techniques can be used for the assessment of wall shear rate and arterial impedance.

The method to estimate non-invasively time dependent wall shear rate is based upon the evaluation of the velocity distribution obtained by means of ultrasound using off-line signal processing. The off-line processing is performed in the RF-domain and consists of an RF-domain velocity estimator preceded by an adaptive vessel wall filter. The latter is adaptive to obtain optimal discrimination between the slowly moving structures, like vessel walls, and the slowly moving blood near the vessel wall. It can be concluded that the proposed method to estimate time dependent wall shear rate in vivo is applicable and reproducible (13\%). However, the method is only reproducible in relatively straight blood vessels because in curved vessels the wall shear rate estimates are affected by the secondary velocities. Because of the limitation in spatial resolution of pulsed ultrasound preference is given to the determination of the maximum spatial derivative of the blood velocity profile. However, this may introduce an error when the velocity gradient near the wall differs from the maximum velocity gradient.

The method to estimate arterial impedance is based upon the evaluation of the spectral components of the normalized distension and center-line blood flow velocity waveforms, both obtained by ultrasound using off-line signal processing. It is demonstrated that for the harmonics between two and five and for Womersley numbers as observed in the common carotid artery center-line blood flow velocity is linear with volume flow and that distension is linear with pressure over a wide frequency range. Therefore, the pressure and volume flow waveforms may be replaced by the distension 
and the center-line blood flow velocity waveforms, respectively. It is concluded that in vitro the proposed method to estimate dimensionless arterial impedance gives the same results as the spectral pressure-flow relationship. The proposed method to estimate noninvasively the dimensionless arterial impedance in vivo has a high coefficient of variation (29\%) for repeated measurements. However, it can not be excluded that this large variation is caused by physiological variation in arterial impedance.

\subsection{Recommendations}

The methods as described in this thesis can be used to study the interrelationship between distension, blood flow velocity, blood viscosity, wall shear rate and arterial impedance. The first results obtained regarding the relationship between wall shear rate, blood viscosity and distension are promising.

One of the most urgent changes to be made to improve the assessment of wall shear rate and arterial impedance is the replacement of the additional emitter/receiver unit by a conventional echo-system generating short emission bursts (to achieve a high axial resolution in velocity assessment) synchronously with a reference clock. The conventional available echo-systems do not provide a pulse repetition frequency (PRF) of ultrasound bursts in echo M-mode in accordance with the anticipated blood flow velocities (up to $10 \mathrm{kHz}$ ). The practical limitations of commercially available echosystems are only partially circumvented by an additional emitter/receiver unit, taking over control of the ultrasound probe once the ultrasound beam has been positioned correctly.

Another point of attention should be that the RF-signals received do not provide information about the projection of the blood flow velocity vector ( $x-, y-, z$-component) on the ultrasound beam. Therefore, to obtain a reliable estimate of wall shear rate using the method proposed, the wall shear rate should be measured in relatively straight blood vessels. To overcome this limitation, algorithms should be developed for the assessment of vectorial blood flow velocity.

Finally, the quality of the discrimination filters should be improved. The adaptive discrimination filter as proposed in this thesis and used in the method for the assessment of wall shear rate performs well. However, it is still necessary to provide information about the origin of a reflection. For example, if for an estimation window the signal to noise ratio of the reflection component is less than $17 \mathrm{~dB}$ or if the velocity of the wall is higher than $10 \mathrm{~mm} / \mathrm{s}$ the adaptation process has to be bypassed. It should be possible to develop an adaptive discrimination filter without the need to use (discrete) thresholds. 


\section{References}

Ahn Y.B., Park S.B. Estimation of mean frequency and variance of ultrasonic Doppler signal by using second-order autoregressive model. IEEE Trans. Ultrason. Ferroelec. and Freq. Contr. UFFC38:172-182; 1991 .

Angeisen B.A., Kristoffersen K. Discrete time estimation of the mean Doppler frequency in ultrasonic blood velocity measurements. IEEE Trans. Biomed. Eng. BME-30:207-214; 1983.

Angelsen B.A.J. Spectral estimation of a narrow-band Gaussian process from the distribution of the distance between adjacent zeroes. IEEE Trans. Biomed. Eng. BME-27:108-110; 1980.

Angelsen B.A.J. A theoretical study of the scattering of ultrasound from blood. IEEE Trans. Biomed. Eng. BME-27:61-67; 1980.

Angelsen B.A.J., Kristoffersen K. On ultrasonic MTI measurement of velocity profiles in blood flow. IEEE Trans. Biomed. Eng. BME-26:665-671; 1979.

Arcaro G., Laurent S., Jondeau G., Hoeks A.P., Safar M.E. Stiffness of the common carotid artery in treated hypertensive patients. J Hypertens. 9:947-954; 1991.

Attinger E., Sugawara H., Navarro A., Riccetto A., Martin R. Pressure-flow relations in dog arteries. Circulation Res. 19:230-245; 1966.

Azaria M., Hertz D. Time delay estimation by generalized cross correlation methods. IEEE Trans. Acoustic Speech Signal Proc. ASSP-32:280-285; 1984.

Baker D.W., Forster F.K., Daigle R.E. Doppler principles and technique. In: Ultrasound: its application in medicine and Biology Part I. F.J. Fry (ed). Amsterdam: Elsevier, 113-129; 1978.

Benetos A., Laurent S., Hoeks A.P., Boutouyrie P.H., Safar M.E. Arterial alterations with aging and high blood pressure. A noninvasive study of carotid and femoral arteries. Arterioscler. Thromb. 13:90-97; 1993.

Bonnefous O., Pesque P. Time domain formulation of pulsed Doppler ultrasound and blood velocity estimation by cross correlation. Ultrasonic Imaging. 8:75-85; 1986.

Brandestini M.A., Eyer M.K., Stevenson J.G. M/Q mode echocardiography - the synthesis of conventional echo with digital miltigate Doppler. In: Echocardiology. C.T. Lancee (ed). The Hague: Martinus Nijhoff, 441-446; 1979.

Brands P.J., Hoeks A.P.G. A comparison method for mean frequency estimators for Doppler ultrasound. Ultrasonic imaging. 14:367-386; 1992.

Brands P.J., Hoeks A.P.G., Hofstra L., Reneman R.S. A non-invasive method to estimate wall shear rate using ultrasound. Ultrasound Med. Biol. 21:171-185; 1995.

Burckhardt C.B. Comparison between spectrum and time interval histogram of ultrasound Doppler signals. Ultrasound Med. Biol. 7:79-82; 1981. 
Callaerts D., De Moor B., Vandewalle J., Sansen W., Vantrappen G., Janssens J. Comparison of SVD methods to extract the foetal electrocardiogram from cutaneous electrode signals. Med. Biol. Eng. Comput. 28:217-224; 1990.

Caro C.G. Mechanical factors in atherogenesis. In: Cardiovascular flow dynamics and measurements. N. Hwang, N. Normann (ed). Baltimore: Univ. Park Press, 473-478; 1977.

Caro C.G., Fitz-Gerald J.M., Schroter R.C. Atheroma and arterial wall shear, observation, correlation and proposal of a shear-dependent mass transfer mechanism for atherogenesis. Proc. R. Soc. London Series B. 47:55-62; 1983.

Cassereau D., Guyomar D. Computation of the impulse diffraction of any obstacle by impuls ray modeling. J Acoust. Soc. Am. 84:1504-1516; 1988.

Chivers R.C., Santosa F. Numerical considerations for interface reflections in medical ultrasound. Phys. Med. Biol. 31:19-37; 1986.

Chon J.N., Finkelstein S., McVeigh G., Morgan D., LeMay L., Robinson J., Mock J. Noninvasive pulse wave analysis for the early detection of vascular desease. Hypertension. 26:503-508; 1995.

Davies P.F. Endothelial cells, hemodynamic forces, and the localization of atherosclerosis. In: Endothelial Cells. U.S. Rayan (ed). Boca Raton, FL: CRC Press, 123-139; 1988.

Davies P.F. How do vascular endothelial cells respond to flow. NIPS. 4:22-25; 1989.

Davies P.F., Barbee K.A. Endothelial cell surface imaging: insights into hemodynamic force transduction. NIPS. 9:153-157; 1994.

Davies P.F., Dewey C.F., Bussolari S.R., Gordon E.J., Gimbrone M.A. Influence of hemodynamic forces on vascular endothelial function: In vitro studies of shear stress and pinocytosis in bovine aortic endothelial cells. J. Clin. Invest. 73:1121-1129; 1983.

De Jong P.G.M., Arts T., Hoeks A.P.G., Reneman R.S. Determination of tissue motion velocity by correlation interpolation of pulsed ultrasonic echo signals. Ultrasonic Imaging. 12:84-98; 1990.

Deng S.X., Tomioka J., Debes J.C., Fung Y.C. New experiments on shear modulus of elasticity of arteries. Am. J Physiol. 266:H1-10; 1994.

Drain L.E. The laser Doppler technique. Chicester: John Wiley \& Sons; 1980.

Dumoulin C.L., Doorly D.J., Caro C.G. Quantitative measurement of velocity at multiple positions using comb excitation and Fourier velocity encoding. Magn. Reson. Med. 29:44-52; 1993.

Duncan D.D., Bargeron C.B., Borchardt S.E., Deters O.J., Gearhart S.A., Mark F.F., Friedman M.H. The effect of compliance on wall shear rate in cats of a human aortic bifurcation. J Biom. Eng. 112:183-188; 1990.

Farrar D.J., Green H.D., Bond M.G., Wagner W.D., Gobbee R.A. Aortic pulse wave velocity, elasticity, and composition in a nonhuman primate model of atherosclerosis. Circulation Res. 43:52-62; 1978. 
Ferrara K.W., Algazi V.R. A new wideband spread target maximum likelihood estimator for blood velocity estimation-Part I: Theorie. IEEE Trans. Ultrason. Ferroelectr. Freq. Contr. 38:1-16; 1991.

Flaherty J.T., Pierce J.E., Ferrans V.J., Patel D.J., Tucker W.K., Fry D.L. Endothelial nuclear patterns in the canine arterial tree with particular reference to hemodynamic events. Circulation Res. 30:23$33 ; 1972$.

Frangos J.A., Eskin S.G., McIntyre L.V., Ives C.L. Flow effects on prostacyclin production by cultured human endothelial cells. Science. 227:1477-1479; 1985.

Frank O. Kritik der elastischen Manometer. Z. Biol. 44:445-613; 1903.

Franklin D.L., Baker D.W., Ellis R.M. A pulsed ultrasonic flowmeter. IRE Trans. Med. Electron. $6: 204 ; 1959$

Friedman M.H. Some atherosclerosis may be a consequence of the normal adaptive vascular response to shear. Atherosclerosis. 82:193-196; 1990.

Friedman M.H., Bargeron C.B., Deters D.J., Hutchins G.M., Mark F.F. Correlation between wall shear and intimal thickness at a coronary artery branch. Atherosclerosis. 68:27-33; 1987.

Friedman M.H., Hutchins G.M., Bargeron C.B., Deters O.J., Mark F.F. Correlation between intimal thickness and fluid shear in human arteries. Atherosclerosis. 39:412-437; 1981.

Friedman M.H., Peters O.J., Bargeron C.B., Hutchins G.M., Mark F.F. Shear-dependent thickening of the human arterial intima. Atherosclerosis. 60:161-171; 1986.

Fung Y.C., Liu S.Q. Elementary mechanics of the endothelium of blood vellels. J Biomechan. Eng. 115:1-12; 1993.

Furchgott R.F., Zawadzki J.V. The obligatory role of endothelial cells in the relaxation of arterial smooth muscle by acetylcholine. Nature. 288:373-6; 1980.

Gibson C.M., Diaz L., Kandarpa K., Sacks F.M., Pasternak R.C., Sandor T., Feldman C., Stone P.H. Relation of vessel wall shear stress to atherosclerois progression in human coronary arteries. Arterioscler. Thromb. 13:310-315; 1993.

Girerd X., Mourad J.J., Acar C., Heudes D., Chiche S., Bruneval P., Mignot J.P., Billaud E., Safar M., Laurent $S$. Noninvasive measurement of medium-sized artery intima-media thickness in humans: in vitro validation. J Vasc. Res. 31:114-20; 1994.

Glagov S., Bassiouny H.S., Giddens D., Zarins C.K. Intimal thickening morphogenesis, functional significance and detection. J. Vasc. Investigation. 1:2-14; 1995.

Gow B.S., Taylor M.G. Measurements of viscoelastic properties of arteries in the living dog. Circulation Res. 23:111-122; 1968.

Hales S. Statical essays including haemastatics. London: Wilson \& Nichol; 1733.

Hansen F., Bergqvist D., Mangell P., Ryden A., Sonesson B., Lanne T. Non-invasive measurement of pulsatile vessel diameter change and elastic properties in human arteries: a methodological study. Clin. Physiol. 13:631-43; 1993. 
Hayoz D., Tardy Y., Perret F., Waeber B., Meister J.J., Brunner H.R. Non-invasive determination of arterial diameter and distensibility by echo-tracking techniques in hypertension. $\mathrm{J}$ Hypertens. 10:S95-100; 1992.

Helmlinger G., Geiger R.V., Schreck S., Nerem R.M. Effects of pulsatile flow on cultured vascular endothelial cell morphology. J Biomech. Eng. 113:123-131; 1991.

Hoeks A.P.G. Non-invasive study of the local mechanical arterial characteristics in humans. In: The arterial system in hypertension. M.E. Safar, M.F. O'Rourke (ed). Dordrecht: Kluwer Academic, 119-134; 1993.

Hoeks A.P.G., Arts G.J., Brands P.J., Reneman R.S. Comparison of the performance of the RF crosscorrelation and Doppler autocorrelation technique to estimate the mean velocity of simulated ultrasound signals. Ultrasound Med. Biol. 19:727-740; 1993.

Hoeks A.P.G., Arts T.G.J., Brands P.J., Reneman R.S. Processing scheme for velocity estimation using ultrasound RF cross correlation techniques. European J Ultrasound. 1:171-182; 1994.

Hoeks A.P.G., Brands P.J., Arts T.G.J., Reneman R.S. Subsample volume processing of Doppler ultrasound signals. Ultrasound Med. Biol. 20:953-965; 1994.

Hoeks A.P.G., Brands P.J., Smeets F.A.M., Reneman R.S. Assessment of the distensibility of superficial arteries. Ultrasound Med. Biol. 16:121-128; 1990.

Hoeks A.P.G., Di X., Brands P.J., Reneman R.S. An effective algorithm for measuring diastolic artery diameters. Archives of Acoustics. 20:65-76; 1995.

Hoeks A.P.G., Peeters H.P.M., Ruissen C.J., Reneman R.S. A novel frequency estimator for sampled Doppler signals. IEEE Trans. Biomed. Eng. BME-31:212-220; 1984.

Hoeks A.P.G., Reneman R.S., Peronneau P.A. A multigate pulsed Doppler system with serial data processing. IEEE Trans. Sonics Ultrason. 28:242-7; 1981.

Hoeks A.P.G., Ruissen C.J., Hick P., Reneman R.S. Transcutaneous detection of relative changes in artery diameter. Ultrasound Med. Biol. 11:51-59; 1985.

Hoeks A.P.G., Van de Vorst J.J.W., Dabekaussen A., Brands P.J., Reneman R.S. An efficient algorithm to remove low frequency Doppler signals in digital Doppler systems. Ultrasonic Imaging. 13:135-144; 1991.

Jensen J.A. Stationary echo canceling in velocity estimation by time-domain cross-correlation. IEEE Trans. Med. Imaging. 12:471-477; 1993.

Jongen H.A.H., Thijssen J.M., Van den Aarssen M., Verhoef W.A. A general model for the absorption of uitrasound by biological tissues and experimental verification. J Accoust. Soc. A. 79:535-540; 1986.

Kalmus H.P. Electronic flowmeter system. Rev. Scient. Instrum. 5:3-6; 1954.

Kamiya A., Bukhari R., Togawa T. Adaptive regulation of wall shear stress optimizing vascular tree function. Bull. Math. Bio. 46:127-137; 1984. 
Kamiya A., Togawa T. Adaptive regulation of wall shear stress to flow change in the canine carotid artery. Am. J Physiol. 239:H14-H21; 1980.

Kaneko Z., Shiraishi J., Omizo H., Kato K., Motomiya M., Izumi T., Okumura T. Analysing blood flow with a sonograph. Ultrasonics. 4:22-33; 1966.

Kasai C., Namekawa K., Koyano A., Omoto R. Real-time two-dimensional blood flow imaging using an autocorrelation technique. IEEE Trans. Sonics Ultrasonics. SU-32:458-464; 1985.

Kay S.M. Modern spectral estimation theory \& application. In: Prentice-hall signal processing series. A.V. Oppenheim (ed). Englewood Cliffs, New Jersey: Prentice Hall; 1988.

Keller H.M., Meier W.E., Anliker M., Kumpe D.A. Noninvasive measurement of velocity profiles and blood flow in the common carotid artery by pulsed Doppler ultrasound. Stroke. 7:370-377; 1976.

Kool M.J.F., Van Merode T., Reneman R.S., Hoeks A.P.G., Struijker Boudier H.A.J., Van Bortel L.M.A.B. Evaluation of reproducibility of a vessel wall movement detector system for assessment of large artery properties. Cardiovasc. Res. 28:610-614; 1994.

Kristoffersen $\mathrm{K}$. Time-domain estimation of the center frequency and spread of Doppler spectra in diagnostic ultrasound. IEEE Trans. Ultrasonics, Ferroelectrics and Frequency Control. UFFC35:685-700; 1988 .

Ku D.N., Giddens D.P., Zarins C.K., Glagov S. Pulsatile flow and atherosclerosis in the human carotid bifurcation. Artheriosclerosis. 5:293-302; 1985.

LaBarbera M. Principles of design of fluid transport systems in zoology. Science. 249:992-1000; 1990.

LaColley P.J., Pannier B.M., Slama M.A., Cuche J.L., Hoeks A.P., Laurent S., London G.M., Safar M.E. Carotid arterial haemodynamics after mild degrees of lower-body negative pressure in man. Clin. Sci. Colch. 83:535-540; 1992.

Laurent S., Caviezel B., Beck L., Girerd X., Billaud E., Boutouyrie P., Hoeks A., Safar M. Carotid artery distensibility and distending pressure in hypertensive humans. Hypertension. 23:878-883; 1994.

Lehmann E.D., Hopkins K.D., Marsden R.M., Brown I., Jones R.L., Turay R.C., Taylor M.G., Gosling R.G. Aortic compliance measured by non-invasive Doppler ultrasound: application of a personal computer based Mk II system and its repeatability. Med. Eng. Phys. 16:213-221; 1994.

Lindstrom K., Marsal K., Gennser G., Bengtsson L., Benthin M., Dahal P. Device for measurement of fetal breathing movements-I: The TD-recorder. A new system for recording the distance between two eçhogenerating structures as a function of time. Ultrasound Med. Biol. 3:143-151; 1977.

Linssen F.M.J., Hoeks A.P.G. Transducer characterization from pressure amplitude distribution measurements using a Kalman filter as parameter estimation algorithm. Ultrasonic Imaging. $12: 309-323 ; 1990$.

Lunt M.J. Accuracy and limitations of the ultrasonic Doppler blood velocitymeter and zero crossing detector. Ultrasound Med. Biol. 2:1-10; 1975. 
Miller K.S., Rochwarger M.M. A covariance approach to spectral moment estimation. IEEE Trans. Inform. Theory. IT-18:558-596; 1972.

Mills C.J., Gabe I.T., Gault J.H., Mason D.T., Ross J., Braunwald J.E., Shillingford J.P. Pressure-flow relationship and vascular impedance in man. Cardiovascular Res. 4:405-417; 1970.

Milnor W.R. Arterial impedance as ventricular afterload. Circ. Res. 36:232-7; 1975.

Milnor W.R. Hemodynamics. Baltimore: Williams \& Wilkins; 1989.

Muijtjens A.M.M., Roos J.M.A., Prinzen T.T., Hasman A., Reneman R.S., Arts T. Noise reduction in estimating cardiac deformation from marker tracks. Am. Physiol. Soc. H599-H605; 1990.

Murgo J.P., Westerhof N., Giolma J.P., Altobelli S.A. Aortic input impedance in normal man: relationship to pressure wave forms. Circulation. 62:105-16; 1980.

Namekawa K., Kasai C., Tsukamoto M. A real-time bloodflow imaging system utilizing autocorrelation techniques. In: Ultrasound. R.A. Lerki, P. Morley (ed). New York: Pergamon Press, 203-208; 1982.

Nerem R.M. Vascular fluid mechanics, the arterial wall, and atherosclerosis. J Biomech. Eng. 114:274$282 ; 1992$.

Nerem R.M., Girard P.R. Hemodynamic influences on vascular endothelial biology. Toxicologic Pathology. 18:572-582; 1990.

Olesen C.P., Clapham D.E., Davies P.F. Haemodynamic shear stress activates a $\mathrm{K}+$ current in vascular endothelial cells. Nature. 331:168-170; 1988.

Oppenheim A.V., Schafer R.W. Digital signal processing. Englewood Cliffs, New Jersey: PrenticeHall; 1975.

O'Rourke M.F. Vascular impedance: the relationship between pressure and flow. In: Arterial function in health and disease. Edinburgh: Churchill Livingstone, 94-132, 185-243; 1982.

O'Rourke M.F., Avolio A.P. Pulsatile flow and pressure in human systemic arteries: studies in man and in multibranched model of the human systemic arterial tree. Circulation Res. 46:363-372; 1980.

O'Rourke M.F., Taylor M.G. Input impedance of the systemic circulation. Circulation Res. 20:365$380 ; 1967$.

O'Rourke M.F., Yaginuma T., Avolio A.P. Physiological and pathophysiological implications of ventricular/vascular coupling. Ann. Biomed. Eng. 12:119-34; 1984.

Pagani M., Mirsky 1., Baig H., Manders W.T., Kerkhof P., Vatner S.F. Effects of age on aortic pressure-diameter and elastic stiffness-stress relationships in unanesthetized sheep. Circulation Res. $44: 420-429 ; 1979$.

Perktold K., Thurner E., Kenner T. Flow and stress characteristics in rigid walled and compliant carotid artery bifurcation models. Med. Biol. Eng. Compt. 32:19-26; 1994. 
Pevec W.C., L'Italien G.J., Megerman J., Cambria R.P., Abbott W.M. Abnormal wall strain at distal end-to-side anastomoses. Ann. Vasc. Surg. 7:14-20; 1993.

Pignoli P., Tremoli E., Poli A., Oreste P., Paoletti R. Intimal plus medial thickness of the arterial wall: a direct measurement with ultrasound imaging. Circulation. 74:1399-406; 1986.

Powalowski $T$. A noninvasive ultrasonic method for vascular input impedance determination applied in diagnosis of the carotid arteries. Arch. Acoustics. 14:293-312; 1989.

Rabiner L.R., Gold B. Theory and application of digital signal processing. Englewood Cliffs, New Jersey: Prentice-Hall; 1975.

Randall J.E., Stacey R.W. Mechanical impedance of the dog's hind leg to pulsatile blood flow. Am. J Physiol. 187:94-98; 1956.

Rashevsky N. Mathematical Biophysics. New York: Pergamon Press; 1960.

Reneman R.S. Cardiovascular applications of ultrasound. Amsterdam: North-Holland Publishing Co.; 1974.

Reneman R.S., Spencer M.P. Difficulties in processing of an analogue Doppler flow signal; with special reference to zero-crossing meters and quantification. In: Cardiovascular applications of ultrasound, R.S. Reneman (ed). Amsterdam-London: North-Holland Publishing Co., 32-42; 1973.

Reneman R.S., van Merode T., Brands P.J., Hoeks A.P. Inhomogeneities in arterial wall properties under normal and pathological conditions. J Hypertens. 10:S35-39; 1992.

Reneman R.S., van Merode T., Hick P., Hoeks A.P. Flow velocity patterns in and distensibility of the carotid artery bulb in subjects of various ages. Circulation. 71:500-509; 1985.

Reneman R.S., van Merode T., Hick P., Hoeks A.P. Cardiovascular applications of multi-gate pulsed Doppler systems. Ultrasound Med. Biol. 12:357-370; 1986.

Reneman R.S., van Merode T., Hick P., Muytjens A.M., Hoeks A.P. Age-related changes in carotid artery wall properties in men. Ultrasound Med. Biol. 12:465-471; 1986.

Rodbard S. Negative feedback mechanisms in the architecture and function of the convective and cardiovascular tissue. Prespective Biol. Med. 13:507-527; 1970.

Rodbard S. Vascular caliber. Cardiology. 60:4-49; 1975.

Ross R. Atherosclerosis: A problem of biology of arterial wall cells and their interaction with blood components. Atherosclerosis. 1:293-311; 1981.

Ross R. Mechanics of atherosclerosis - A review. Adv. Nephrol. 19:79; 1990.

Ross R., Glomset J. The pathogenesis of atherosclerosis. England J Med. 295:369-377; 1976.

Rubanyi G.M., Freay A.D., Kauser K., Johns A., Harder D.R. Mechanoreception by the endothelium: mediators and mechanisms of pressure- and flow-induced vascular response. Blood vessels. 27:246$257 ; 1990$. 
Ryan U.S., Ryan J.W., Whitaker C. How do kinins affect vascular tone? Adv. Exp. Med. Biol. 10:121$127 ; 1979$.

Schwartz C.J., Mitchell K.R.A. Observation on localizations of arterial plaques. Circulation Res. 11:63-73; 1972.

Schwartz C.J., Valente A.J., Sprague E.A., Kelley J.L., Nerem R.M. The pathogenesis of atherosclerosis: An overview. Clinical Cardiology. 14:1-16; 1991.

Shanmugan K.S., Breipohl A.M. Random signals: detection estimation and data analysis. New York: John Wiley \& Sons; 1988.

Simon A.C., Levenson J., Flaud P. Pulsatile flow and oscillating wall shear stress in the brachial artery of normotensive and hypertensive subjects. Cardiovasc. Res. 24:129-136; 1990.

Smith P.H. Longitudinal extension of the pulmonary trunk during breathing in young rabbits: a possible factor in elastin fragmentation. Can. J Physiol. Pharmacol. 54:113-7; 1976.

Sonesson B., Hansen F., Stale H., Lanne T. Compliance and diameter in the human abdominal aorta-the influence of age and sex. Eur. J Vasc. Surg. 7:690-7; 1993.

Stegall H.F., Kardon M.B., Kemmerer W.T. Indirect measurement of arterial blood pressure by Doppler ultrasonic sphygmomanometry. J Appl. Physiol. 25:793-798; 1968.

Tardy Y., Hayoz D., Mignot J.P., Richard P., Brunner H.R., Meister J.J. Dynamic non-invasive measurements of arterial diameter and wall thickness. J Hypertens. 10:S105-109; 1992.

Tysoe C., Evans D.H. Bias in mean frequency estimation of Doppler signals due to wall clutter filters. Ultrasound Med. Biol. 21:671-677; 1995.

van Leeuwen G.H., Hoeks A.P.G., Reneman R.S. Simulation of real-time frequency estimators for pulsed Doppler systems. Ultrasonic Imaging. 8:252-271; 1986.

van Merode T., Hick P.J., Hoeks A.P., Smeets F.A., Reneman R.S. Differences in carotid artery wall properties between presumed healthy men and women. Ultrasound Med. Biol. 14:571-574; 1988.

Webster J.G. Medical instrumentation. Boston: Houghton Mifflin; 1978.

Wells P.N.T. The possibility of harmful biological effects in ultrasonic diagnosis. In: Cardiovascular applications of ultrasound., R.S. Reneman (ed). Amsterdam, the Netherlands: North-holland Publishing Company, 1-17; 1974.

Westerhof N., Elzinga G. The relation between end-diastolic volume and source impedance of the left ventricle. Arch. Int. Physiol. Biochim. 82:326-9; 1974.

Westerhof N., Noordergraaf A. Arterial viscoelasticity: a generalized model effect on input impedance and wave travel in the system tree. J Biomechanics. 3:357-379; 1970.

White K.C., Kavanaugh J.F., Wang D.M., Tarbell J.M. Hemodynamics and wall shear rate in the abdominal aorta of dogs. Circulation Res. 75:637-649; 1994.

Wiggers C.J. The pressure pulses in the cardiovascular system. M. Longman (ed). London; 1928. 
Willemetz J.C., Nowicki A., Meister J.J. Bias and variance in the estimate of the Doppler frequency induced by a wall motion filter. Ultrasonic Imaging. 11:215-225; 1989.

Wilson L.S. Description of broad-band pulsed Doppler ultrasound processing using the twodimensional Fourier transform. Ultrasonic Imaging. 13:301-315; 1991.

Wilson L.S., Gill R.W. Measurement of two-dimensional blood velocity vectors by the ultrasonic speckle projection technique. Ultrasonic Imaging. 15:286-303; 1993.

Womersley J.R. An elastic tube theory of pulse transmission and oscillatory flow in mammalian arteries. Wright air development center, Technical report WADC-TR56-614. 1957.

Woodcock J.P. Development of the ultrasonic flowmeter. Ultrasound Med. Biol. 2:11-18; 1975.

Zamir M., Sinclair P., Wonnacott T.M. Relation between diameter and flow in major branches of the aorta. J Biomech. 25:1303-1310; 1992.

Zarins C.K., Zatina M.A., Giddens D.P., Ku D.N., Glagov S. Shear stress regulation of artery lumen diameter in experimental atherogenesis. J Vasc. Surg. 5:413-420; 1987. 


\section{Summary}

The principal aim of this study was the development and implementation of noninvasive methods for the assessment of wall shear rate and arterial impedance by means of ultrasound. Wall shear rate is the near wall velocity gradient with respect to the radial position. Arterial impedance describes the opposition to pulsatile blood volume flow and is defined as the ratio of the spectral components of local pressure and local volume flow. Non-invasive assessment by means of ultrasound is the key to study hemodynamic parameters because it will not interfere with the physiological parameter(s) to measure. The assessment of wall shear rate demands accurate assessment of blood flow velocity profiles, while the assessment of arterial impedance demands the simultaneous and local assessment of center-line blood flow velocity and the diameter chance of a blood vessel (distension). The accurate assessment of velocity profiles or the simultaneous assessment of distension and center-line blood flow velocity waveforms is possible with the use of radio frequency (RF) domain signal processing algorithms (Section 1.4.5) applied to ultrasound RF-signals (Section 1.4.3) acquired (Section 5.3) in echo system motion mode (M-mode) or Doppler-mode setting. The M-mode is preferred because of its higher resolution along the ultrasound beam. The RF-domain signal processing necessary to extract the time dependent information from the RF-signals received consists of discrimination of the different ultrasound RFsignal components (reflections, scattering and noise) by means of a discrimination filter, whereafter the temporal mean frequency of the different components is estimated by means of a mean frequency estimator. The temporal mean frequency of the reflection component in the RF-signals received is related to tissue velocity (vessel wall motion), while the temporal mean frequency of the scattering component (red blood cells) is related to blood flow velocity.

Chapter 1 gives an introduction to the global principles of ultrasound signal processing necessary for the assessment of distension and blood flow velocity profiles. Moreover, a brief description of the vascular system, the properties of the blood vessel wall and the employment of ultrasound for the assessment of hemodynamic variables is given.

The development of non-invasive methods for the assessment of wall shear rate and arterial impedance is concentrated on a few issues.

The first issue involves the introduction of mathematical principles (Section 2.3) and comparison (Section 2.4) of mean frequency estimators used in ultrasound applications for the assessment of blood flow velocity and/or tissue motion. There are two main classes of estimators: the model-based (or parametric) and model-free (or nonparametric) estimators. The parametric estimator assumes a model with a few characteristic parameters, while the nonparametric estimator assumes characteristic properties of a given process. Most of the mean frequency estimators employed in ultrasound applications are parametric estimators. From the class of parametric estimators it is the RF-domain cross-correlation model (CCM) estimator (Section 2.3.8) 
(De Jong et al. 1990) that exhibits the best quality in the assessment of blood flow velocity and tissue motion (Hoeks et al. 1993).

The second issue concerns the principles of discrimination filters (Chapter 3 ) and the effect of these filters on the velocity estimation range (Chapter 4). Discrimination filters are necessary because $\mathrm{RF}$-signals are composed of three major signal components, i.e., reflections, scattering, and noise (Section 1.4.5). To estimate (blood flow) velocity and (tissue) motion from the scattering and reflections received, it is necessary to discriminate between these RF-signal components. The discrimination, based on the difference in temporal properties of the RF-signal components, can be obtained with two classes of filters: static filters, having fixed filter characteristics, and dynamic filters, having adaptive filter characteristics (Section 3.3.5). The filter characteristics of a static filter are based on assumptions of signal properties, while dynamic filter characteristics are based on estimates of dynamic signal properties. The two classes of discrimination filters can be applied to both the RF-domain and the LF-domain (low frequency domain) (Section 2.3.1).

The third issue concerns the implementation of an adaptive discrimination filter in combination with the RF-domain cross-correlation model (CCM) estimator for the assessment of wall shear rate (Chapter 5). To determine wall shear rate from velocity profiles accurately, one must be able to assess unambiguously low blood flow velocities near the vessel wall. An important limitation of wall shear rate assessment by means of ultrasound is the discrimination between signals induced by slowly moving structures (reflections), like vessel walls, and those induced by slowly moving blood near the vessel wall (scattering). To overcome this limitation an adaptive discrimination filter was introduced to determine accurately near-wall flow velocity behavior. It was demonstrated that dynamic filtration, in combination with the CCM estimator, exhibits the best performance in the assessment of low (near wall) blood flow velocities (Chapter 4). The validation of wall shear rate assessment by means of ultrasound was performed through two in vivo studies (Chapter 5, Chapter 6) and one in vitro study (Chapter 6). The in vivo study in Chapter 5 concerns a pilot study of the reproducibility of peak wall shear rate assessment $(5 \%)$ in comparison with the reproducibility of peak center-line blood flow velocity assessment $(9 \%)$. The in vivo study in Chapter 6 concerns the comparison and reproducibility of mean wall shear rate $(13 \%)$ in two subject populations of different categories of age. The in vitro study in Chapter 6 concerns a comparison in difference $(9.4 \%)$ between wall shear rate assessed with ultrasound and with laser Doppler anemometry (LDA).

Finally a method was developed for the assessment of dimensionless arterial impedance by means of ultrasound (Chapter 7). Arterial impedance describes the relationship between the pressure and volume flow waveforms in the frequency domain. However, pulsatile pressure and volume flow waveforms, simultaneously recorded at the same location, are difficult to obtain non-invasively. The non-invasive assessment of dimensionless arterial impedance presented in this thesis is based on the ratio of the 
harmonics of the normalized distension and normalized center-line blood flow velocity waveforms (Section 7.2) because both waveforms can be obtained non-invasively and simultaneously at the same location by means of ultrasound. The validation of this method for the assessment of dimensionless arterial impedance was performed through an in vitro study (Section 7.5) and an in vivo study (Chapter 8). The in vivo validation involved the reproducibility (29\%) and the effect of age on arterial impedance. In agreement with the age related change in arterial wall properties there is a significant change with age in the non-invasive estimated arterial impedance. The in vitro study pertains to a comparison of the dimensionless ultrasound arterial impedance with the dimensionless spectral relationship of pressure and volume flow.

The thesis is concluded with a general discussion (Chapter 9). 


\section{Samenvatting}

De doelstelling van deze studie was de ontwikkeling en implementatie van niet-invasive meetmethoden voor de schatting van wandafschuifsnelheid en arteriële impedantie m.b.v. ultrageluid. Wandafschuifsnelheid is de snelheidsgradiënt (met betrekking tot de radiële positie) bij de vaatwand. Arteriële impedantie vormt de beschrijving van de tegenwerking (wrijving, traagheid, compliantie en weerstand) van de bloedvolumestroom en is gedefinieerd als de verhouding tussen de spectrale componenten van lokale bloeddruk en lokale bloedvolumestroom. Niet-invasive schatting $\mathrm{m}$.b.v. ultrageluid is de sleutel tot de studie van hemodynamische parameters omdat dit de fysiologische condities van de te meten parameter(s) niet of nauwelijks beïnvloedt. De schatting van wandafschuifsnelheid vereist een nauwkeurige schatting van bloedsnelheidsprofielen, terwijl de schatting van arteriële impedantie een nauwkeurige schatting van de simultaan en lokaal geobserveerde centrale bloedstroomsnelheid (snelheid in het midden van het vat) en verandering in diameter (distensie) als functie van tijd vereist. Deze nauwkeurige schatting van bloedsnelheidsprofielen of de simultane schatting van distensie en centrale bloedstroomsnelheid is mogelijk m.b.v. signaalverwerkingsalgoritmen in het radio frequente (RF) domein (Paragraaf 1.4.5) toegepast op ultrageluids RF-signalen (Paragraaf 1.4.3), verkregen (Paragraaf 5.3) met een echosysteem in bewegingsmode (M-mode) of Doppler-mode. De RF-domein signaalverwerking nodig voor het verkrijgen van tijdafhankelijke informatie uit de ontvangen ultrageluids RF-signalen bestaat uit het onderscheid maken tussen de verschillende ultrageluids RF-signaal componenten (reflectie, verstrooiing en ruis) m.b.v een discriminatie filter, waarna de tijdafhankelijke gemiddelde frequentie van de verschillende componenten kan worden geschat m.b.v. een gemiddelde frequentie schatter. De tijdafhankelijke gemiddelde frequentie van de reflectie component in de ontvangen RF-signalen is gerelateerd aan structuursnelheid (vaatwand), terwijl de tijdafhankelijke gemiddelde frequentie van de verstrooiingscomponent (rode bloedcellen) gerelateerd is aan de bloedstroomsnelheid.

Hoofdstuk 1 geeft een introductie van de globale principes van ultrageluidssignaalverwerking noodzakelijk voor het inschatten van distensie (verandering in diameter) en bloedstroomsnelheid. Bovendien wordt er een korte beschrijving gegeven van het bloedvatenstelsel, de eigenschappen van bloedvaten en de toepassing van ultrageluid voor het inschatten van hemodynamische parameters.

De ontwikkeling van de niet-invasive meetmethoden voor wandafschuifsnelheid en arteriële impedantie is beschreven aan de hand van een aantal thema's.

Het eerste thema betreft de introductie van de mathematische principes en de onderlinge vergelijking van gemiddelde frequentieschatters (Hoofdstuk 2) zoals deze worden toegepast in ultrageluidsapplicaties voor het inschatten van bloedstroomsnelheid en/of weefselbeweging. Er zijn twee hoofdklassen van schatters: de model gebaseerde (of parametrische) en de niet model gebaseerde (of niet parametrische) schatters. De 
parametrische schatters veronderstellen een model met een paar karakteristieke parameters, terwijl de niet parametrische schatters zijn gebaseerd op karakteristieke eigenschappen van een gegeven proces. De meeste gemiddelde frequentieschatters in ultrageluids applicaties zijn parametrische schatters. Binnen de klassen van prametrische schatters is het de RF-domein kruiscorrelatie model (CCM) schatter (Paragraaf 2.3.8) (De Jong et al. 1990) welke de beste kwaliteit bezit voor het inschatten van bloedstroomsnelheid en weefselbeweging (Hoeks et al. 1993).

Het tweede thema betreft de principes van discriminatie filters (Hoofdstuk 3) en het effect van deze filters op het bereik van de snelheidschatting (Hoofdstuk 4). Discriminatie filters zijn noodzakelijk omdat de ontvangen RF-signalen zijn opgebouwd uit een drietal signaal componenten namelijk: reflecties (weefsel en bloedvaten), verstrooiing (rode bloedcellen) en ruis (Paragraaf 1.4.5). Voor het inschatten van (bloedstroom)snelheid en (weefsel)beweging uit de ontvangen verstrooiing en reflecties is het noodzakelijk om deze RF-signaal componenten te scheiden. De discriminatie, gebaseerd op de verschillen in tijdafhankelijke eigenschappen van de RF-signaal componenten, kan worden bereikt met twee klassen van filters namelijk: statische filters en dynamische filters (Paragraaf 3.3.5). De statische filters zijn gebaseerd op aannames betreffende RF-signaal eigenschappen, terwijl de dynamische filters dynamisch reageren op veranderingen (adaptief) in RF-signaal eigenschappen d.m.v. schatting. Beide klassen van filters kunnen worden toegepast in het RF-domein en in het LFdomein (laag frequente domein) (Paragraaf 2.3.1).

Het derde thema betreft de implementatie van een dynamisch discriminatie filter in combinatie met de RF-domein kruiscorrelatie model (CCM) schatter voor de inschatting van wandafschuifsnelheid (Hoofdstuk 5). Om nauwkeurig de wandafschuifsnelheid te bepalen uit snelheidsprofielen is het noodzakelijk om lage bloedstroomsnelheden nabij de bloedvatwand te kunnen schatten. Een belangrijke beperking in de schatting van wandafschuifsnelheid m.b.v ultrageluid wordt gevormd door de moeilijkheid in het onderscheiden van signalen afkomstig van langzaam bewegende structuren (reflecties van vaatwand) en signalen afkomstig van langzaam bewegend bloed (verstrooiing van rode bloedcellen) nabij de vaatwand. Om deze beperking te ondervangen is een dynamisch discriminatie filter geïntroduceerd. In hoofdstuk 4 is aangetoond dat een dynamisch filter in combinatie met de CCM schatter de beste eigenschappen bezit voor het inschatten van lage bloedstroomsnelheden nabij de vaatwand. De kwaliteit van het inschatten van afschuifsnelheid m.b.v. ultrageluid is onderzocht d.m.v. twee in-vivo studies (Hoofdstuk 5, Hoofdstuk 6) en een in-vitro studie (Hoofdstuk 6). De in-vivo studie in hoofdstuk 5 betreft de vergelijking in reproduceerbaarheid tussen piek wandafschuifsnelheid (5\%) en piek centrale bloedstroomsnelheid $(9 \%)$. De in-vivo studie in hoofdstuk 6 betreft de vergelijking en reproduceerbaarheid van gemiddelde wandafschuifsnelheid $(13 \%)$ in een populatie van twee groepen met verschillende leeftijd. De in-vitro studie in hoofdstuk 6 betreft het verschil in wandafschuifsnelheid schatting (9.4\%), gemeten m.b.v. ultrageluid en laser-Doppler. 
Het laatste thema betreft de ontwikkeling van een methode voor het inschatten van dimensieloze arteriële impedantie m.b.v. ultrageluid (Hoofdstuk 7). Arteriële impedantie beschrijft de relatie tussen lokale bloeddruk en lokale bloedvolumestroom in het frequentie domein. Het simultaan niet-invasief meten van lokale bloeddruk en lokale bloedvolumestroom op dezelfde lokatie is moeilijk uitvoerbaar. De niet-invasive schatting van dimensieloze arteriële impedantie zoals gepresenteerd in dit proefschrift is gebaseerd op de ratio van de golfvormen van de harmonische componenten van distensie en centrale bloedstroom-snelheid (Paragraaf 7.2). Beide golfvormen zijn m.b.v. ultrageluid simultaan en niet-invasief the schatten op dezelfde locatie. De kwaliteit van de schattingsmethoden voor dimensieloze arteriële impedantie m.b.v. ultrageluid is onderzocht in een in-vivo studie (Hoofdstuk 8 ) en in een in-vitro studie (Paragraaf 7.5). De in-vivo studie betreft de reproduceerbaarheid (29\%) en het effect van leeftijd op dimensieloze arteriële impedantie. In overeenstemming met de leeftijd gerelateerde veranderingen in de eigenschappen van bloedvaten is er ook een significante verandering met de leeftijd in de dimensieloze arteriële impedantie. De invitro studie betreft een vergelijking van impedantie zoals geschat m.b.v. ultrageluid en berekend op basis van gemeten lokale druk en lokale volumestroom.

Dit proefschrift besluit met een algemene discussie (Hoofdstuk 9). 


\section{Nawoord}

Het onderzoek naar niet-invasieve meetmethoden voor wandafschuifsnelheid en dimensieloze arteriêle impedantie m.b.v. ultrageluid, beschreven in dit proefschrift, vormt een bijdrage in het veelomvattende onderzoeksgebied naar de vragen omtrent de stroming van het bloed in de bloedvaten. De totstandkoming van dit proefschrift is mogelijk geworden door de medewerking en het enthousiasme van een groot aantal mensen.

In de eerste plaats mijn gezin. Mireille, ondanks het feit dat een groot deel van de zorg voor ons gezin op jouw schouders terecht kwam, heb je me altijd volledig gesteund en gezorgd voor een fijne sfeer. Amadeo en Sam, jullie afleiding zorgde voor de nodige ontspanning, waardoor ik alles steeds weer goed kon relativeren.

De basis van dit onderzoek werd gelegd door Arnold Hoeks en Rob Reneman.

Beste Arnold, ik wil je hartelijk bedanken voor de hulp en kennis die je me hebt gegeven bij de uitvoering van het onderzoek en vooral het schrijven van dit proefschrift. Arnold, je vroeg me in de nazomer van 1991 of ik geen interesse had om te promoveren. In eerste instantie dacht ik: wil ik dat wel en kan ik dat wel? Over dat laatste bestond bij jouw geen enkele twijfel. Dit vertrouwen was voor mij een grote stimulans gedurende het promotie onderzoek.

Beste Rob, jij was in de eerste jaren van het onderzoek de enthousiaste man op de achtergrond. In de schrijffase heb ik veel van je geleerd, vooral door de kritische manier waarop je naar een tekst kijkt en de kwaliteit welke je daarvan verlangt. Rob, ik ben je dankbaar voor het enthousiasme, de inspiratie en de prettige sfeer van jouw begeleiding.

Verder wil ik Joost Bremer (Prof. Medische Psychologie), mijn schoonvader, bedanken voor zijn aanmoediging bij het schrijven van dit proefschrift. Joost, jij hebt ieder hoofdstuk gelezen en voorzien van de nodige suggesties en kanttekeningen, daardoor is het zeker ook voor niet-ultrageluidmensen leesbaar(der) geworden. De manier waarop jij die toch vooral technische beschrijvingen wist te begrijpen, oogstte bij mij grote bewondering.

De ontwikkeling van de meetmethoden voor wandafschuifsnelheid werd door Leo Hofstra vanuit de kliniek op de voet gevolgd. Hij was vol ongeduld om de methoden te evalueren en toe te passen. Later heeft deze evaluatie geresulteerd in een onderzoeksproject dat wordt uitgevoerd door Steven Samijo. Steven is met een groot enthousiasme begonnen aan een aantal klinische studies betreffende de relatie tussen distensie, diameter en wandafschuifsnelheid. Hierbij is de ondersteuning van Jean Willegers van groot belang. Zijn heldere visie en het frisse enthousiasme van Steven, vormen de juiste combinatie om de methoden op een verantwoorde manier toe te passen in de klinische praktijk. 
De in-vitro evaluatie van de meetmethoden vond plaats by de vakgroep fundamentele werktuigbouwkunde van de Technische Universiteit Eindhoven. Het was Marcel Rutten die zorg droeg voor de dynamische werksfeer aldaar. Met zeer goede herinnering kijk ik terug op de tijd van experimenteren. Hierbij denk ik aan de prettige werksfeer en de problemen die altijd werden overwonnen. Marcel, mijn dank voor jouw inzet.

Als laatste wil ik al de stagiaires en afstudeerders bedanken voor hun inzet op het ultrageluidslab. Jongens, jullie leverden en leveren een niet te onderschatten bijdrage. 
Curriculum vitae

\section{Curriculum vitae}

1959

: 22 maart, geboren te Steenbergen

1972-1976 : LTS-Elektrotechniek, Roosendaal

Diploma, Elektricien

1976-1979 : MTS-Elektrotechniek, Breda afstudeerrichting Elektronica

1979-1983 : HTS-Elektrotechniek, Breda afstudeerrichting Technische Computerkunde (TCK)

1983-1987 : Projectmedewerker Stichting Technische Wetenschappen (STW), vakgroep Biofysica, $\mathrm{RL}$ te Maastricht

1987-heden : Fulltime onderzoeksmedewerker vakgroep Biofysica, $\mathrm{RL}$ te Maastricht

$1988 \quad$ : Amadeo

$1992 \quad:$ Sam 


\section{Publications}

Brands P.J., Hoeks A.P.G. A comparison method for mean frequency estimators for Doppler ultrasound. Ultrasonic imaging. 14:367-386; 1992.

Brands P.J., Hoeks A.P.G., Hofstra L. Een niet-invasieve methode voor shear rate meting. Ultrasonoor Bulletin. 33-34; 1993.

Brands P.J., Hoeks A.P.G., Hofstra L., Reneman R.S. A non-invasive method to estimate wall shear rate using ultrasound. Ultrasound Med. Biol. 21:171-185; 1995.

Brands P.J., Hoeks A.P.G., Reneman R.S. The effect of echo suppression on the mean velocity estimation range of the RF cross-correlation model estimator. Ultrasound Med. Biol. 21:945-959; 1995.

Brands P.J., Hoeks A.P.G., Rutten M.C.M., Reneman R.S. A non-invasive method to estimate arterial impedance by means of the assessment of the local diameter change and the local center-line blood flow velocity using ultrasound. Ultrasound Med. Biol. (in Press); 1996.

Hoeks A.P., Arts T.G., Brands P.J., Reneman R.S. Comparison of the performance of the RF crosscorrelation and Doppler auto-correlation technique to estimate the mean velocity of simulated ultrasound signals. Ultrasound Med. Biol. 19:727-40; 1993.

Hoeks A.P., Dabekaussen A., Brands P.J., Reneman R.S. Teclınical limitations of the present color coded US-Doppler systems in analyzing flow anomalies in arteries. VASA. S-32:72-84; 1991.

Hoeks A.P.G., Arts G.J., Brands P.J., Reneman R.S. Comparison of the performance of the RF crosscorrelation and Doppler auto-correlation technique to estimate the mean velocity of simulated ultrasound signals. Ultrasound Med. Biol. 19:727-740; 1993.

Hoeks A.P.G., Arts T.G.J., Brands P.J., Reneman R.S. Processing scheme for velocity estimation using ultrasound RF cross correlation techniques. European J Ultrasound. 1:171-182; 1994.

Hoeks A.P.G., Brands P.J., Arts T.G.J., Reneman R.S. Subsample volume processing of Doppler ultrasound signals. Ultrasound Med. Biol. 20:953-965; 1994.

Hoeks A.P.G., Brands P.J., Reneman R.S. Assessment of arterial distension waveform using Doppler signal processing. J Hypertension. 10 (S6):S19-S22; 1992.

Hoeks A.P.G., Brands P.J., Reneman R.S. Effect of sample window length on the correlation between RF-signal and pulsed Doppler signal intensity. Uitrasound Med. Biol. 20:35-40; 1994.

Hoeks A.P.G., Brands P.J., Smeets F.A.M., Reneman R.S. Assessment of the distensibility of superficial arteries. Ultrasound Med. Biol. 16:121-128; 1990.

Hoeks A.P.G., Di X., Brands P.J., Reneman R.S. An effective algorithm for measuring diastolic artery diameters. Arch. Acoustics. 20:65-76; 1995.

Hoeks A.P.G., Samijo S.K., Brands P.J., Reneman R.S. Assessment of wall shear rate in humans: an ultrasound study. J Vasc. Invest. 1:108-117; 1995. 
Hoeks A.P.G., Samijo S.K., Brands P.J., Reneman R.S. Noninvasive determination of shear-rate distribution across the arterial lumen. Hypertension. 26:26-33; 1995.

Hoeks A.P.G., van de Vorst J.J.W., Dabekaussen A., Brands P.J., Reneman R.S. An efficient algorithm to remove low frequency Doppler signals in digital Doppler systems. Ultrasonic Imaging. 13:135$144 ; 1991$.

Linssen F.M.J., Hoeks A.P.G., Brands P.J. The use of narrowband ultrasound for the characterization of tissue in a thin layer. Phys. Med. Biol. 36:1319-1330; 1991.

Reneman R.S., Brands P.J., Samijo S., Hoeks A.P.G. Non-invasive estimation of wall shear rate in humans by means of ultrasound. Arch Acoustics. 20:57-63;1995.

Reneman R.S., van Merode T., Brands P.J., Hoeks A.P. Inhomogeneities in arterial wall properties under normal and pathological conditions. J Hypertension. 10 (S6):S35-39; 1992.

van Merode T., Brands P.J., Hoeks A.P., Reneman R.S. Faster ageing of the carotid artery bifurcation in borderline hypertensive subjects. J Hypertension. 11:171-176; 1993.

van Merode T., Brands P.J., Hoeks A.P.G., Reneman R.S. Different effects of ageing on elastic and muscular arterial bifurcations in men. J Vasc. Res. 33:47-52; 1996.

van Merode T., Hoeks A.P.G., Brands P.J., Reneman R.S. Local inhomogeneities in wall distensibility in the carotid artery bifurcation in borderline hypertensives. J Hypertension. 10 (S6):118-119; 1992. 